LUCIANO MENDES CAMILLO

ESTUDO DO PONTO INVARIANTE COM A TEMPERATURA ("ZERO TEMPERATURE COEFFICIENT") EM TRANSISTORES SOI MOSFET FABRICADOS COM TECNOLOGIA ULTRA-SUBMICROMÉTRICA 
LUCIANO MENDES CAMILLO

\section{ESTUDO DO PONTO INVARIANTE COM A TEMPERATURA ("ZERO TEMPERATURE COEFFICIENT") EM TRANSISTORES SOI MOSFET FABRICADOS COM TECNOLOGIA ULTRA-SUBMICROMÉTRICA}

Tese apresentada à Escola Politécnica da Universidade de São Paulo para a obtenção do Título de Doutor em Engenharia. 
LUCIANO MENDES CAMILLO

\section{ESTUDO DO PONTO INVARIANTE COM A TEMPERATURA ("ZERO TEMPERATURE COEFFICIENT") EM TRANSISTORES SOI MOSFET FABRICADOS COM TECNOLOGIA ULTRA-SUBMICROMÉTRICA}

Tese apresentada à Escola Politécnica da Universidade de São Paulo para a obtenção do Título de Doutor em Engenharia.

Área de Concentração: Microeletrônica

Orientador:

Prof. Dr. João Antonio Martino 
Este exemplar foi revisado e alterado em relação à versão original, sob responsabilidade única do autor e com a anuência de seu orientador.

São Paulo, de março de 2011.

Assinatura do autor

Assinatura do orientador

\section{FICHA CATALOGRÁFICA}

Camillo, Luciano Mendes

Estudo do ponto invariante com a temperatura ("'Zero Temperature Coefficient"') em transistores SolU Mosfet fabricados com tecnologia ultra-submicrométrica / L.M. Camillo. -- ed.rev. -São Paulo, 2011.

$142 \mathrm{p}$.

Tese (Doutorado) - Escola Politécnica da Universidade de São Paulo. Departamento de Engenharia de Sistemas Eletrônicos.

1. Semicondutores 2. Transistores 3. Alta temperatura 4. Microeletrônica I. Universidade de São Paulo. Escola Politécnica. Departamento de Engenharia de Sistemas Eletrônicos II. t. 


\section{DEDICATÓRIA}

Dedico este trabalho a minha esposa Clara, que sempre me apoiou em todos os momentos bons e ruins, me incentivando e dando suporte para a realização deste trabalho. Aos meus pais Isaias e Regina, que me propiciaram ensinamentos e me mostraram os valores da vida honestidade, integridade e caráter Amo vocês! 


\section{AGRADECIMENTOS}

Ao orientador Prof. Dr. João Antonio Martino, pelo apoio, confiança, amizade, atenção, paciência, e pela oportunidade da realização deste trabalho.

A minha esposa Clara, meus pais e a meu irmão Adriano pelo apoio, carinho, incentivo e compreensão nos momentos difíceis e de ausência, vocês são tudo na minha vida.

A amiga, Carolina D. G. dos Santos pela ajuda, incentivo, confiança, companheirismo.

Aos amigos Milene Galeti, Michele Rodrigues e Vinícius Heltai pelo incentivo, discussões, e apoio durante esta jornada.

Ao professores e amigos Dr. Salvador P. Gimenes, Dr. Aparecido S. Nicolett e Dr. Marcelo A. Pavanello pelo incentivo desse e outros trabalhos.

Aos colegas grupo SOI-CMOS pelo incentivo, discussões, e apoio durante este trabalho.

Ao Laboratório de Sistemas Integráveis da EPUSP pela infra-estrutura oferecida e que permitiu a realização deste trabalho.

À meus familiares que me apoiaram e torceram por mim.

Aos colegas do LSI pelo apoio e discussões, e a todos que direta ou indiretamente colaboraram na execução desse trabalho e que involuntariamente foram aqui omitidos. 


\section{RESUMO}

Neste trabalho é apresentado um estudo do ponto ZTC ("Zero Temperature Coefficient") em dispositivos SOI MOSFETs, funcionando em modo parcialmente (PD-SOI) e totalmente (FD-SOI) depletados. O estudo é realizado a partir de um modelo analítico simples, proposto para determinação da tensão de polarização da porta do transistor no ponto ZTC ( $\left.\mathrm{V}_{\mathrm{ZTC}}\right)$, através dos modelos de primeira ordem das

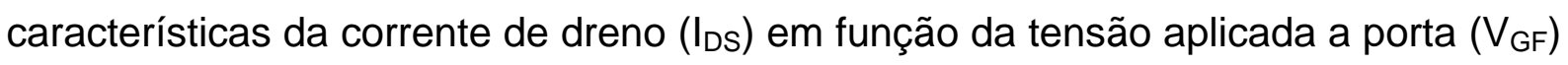
do transistor, considerando as regiões de operação linear e de saturação.

Para a validação do modelo, os resultados obtidos são confrontados com dados experimentais, e foi obtido um bom ajuste dos valores, apesar das simplificações adotadas para o modelo proposto.

Foi realizada uma análise para estudar o impacto no valor de $\mathrm{V}_{\mathrm{ZTC}}$ com a variação no valor de parâmetros de referência, como a concentração de portadores $\left(\mathrm{N}_{\mathrm{af}}\right)$ e a espessura do óxido de porta ( $\left.\mathrm{t}_{\mathrm{oxf}}\right)$. O erro máximo observado em $\mathrm{V}_{\mathrm{ZTC}}$, impondo a variação nos parâmetros $\mathrm{N}_{\mathrm{af}}$ e $\mathrm{t}_{\mathrm{oxf}}$, para os dispositivos PD é de 3,1\% e 4,6\% na região linear, respectivamente; e 3,5\% e 7,2\% na região de saturação, respectivamente. Para os dispositivos FD o erro máximo observado, devido a variação nos parâmetros $\mathrm{N}_{\mathrm{af}}$ e $\mathrm{t}_{\mathrm{oxf}}$, foi de $11 \%$ e $10 \%$ operando no regime linear, respectivamente e $5,3 \%$ e $8,4 \%$ no regime de saturação, respectivamente.

Através do modelo proposto foi realizado o estudo da estabilidade do ponto ZTC em função da variação da degradação da mobilidade com a temperatura (fator $c)$, comprimento de canal $(L)$ e a tensão de dreno $\left(V_{D S}\right)$ para os dispositivos supracitados. A analise da influência do fator $c$ em $V_{\mathrm{ZTC}}$ mostrou-se mais importante nos dispositivos parcialmente depletados (PD).

A tensão $V_{Z T C}$, para os dispositivos nMOS, apresentou um menor valor operando na região de saturação, e torna-se mais pronunciada essa diferença para dispositivos com menor comprimento de canal, para ambos os tipos de dispositivos. Observando a variação de $V_{\text {ZTC }}$ com $V_{D S}$, nota-se uma diminuição no valor de $V_{\text {ZTC }}$ para altos valores de $V_{D S}$, para os dois tipos de dispositivos estudados, $n$ e pMOS.

Os resultados do modelo proposto foram avaliados com dados experimentais de outras tecnologias SOI MOSFET. Também foi obtido um bom ajuste com os valores para as tecnologias GC-SOI e GC-GAA SOI, operando em regime linear e saturação. 
Palavras-chave: Tecnologia SOI. Alta temperatura. Ponto Invariante com a Temperatura. 


\section{ABSTRACT}

This paper presents a study of ZTC point ("Zero Temperature Coefficient) in SOI MOSFETs devices, partially (PD-SOI) and fully (FD-SOI) depleted mode. The study is performed from a simple analytical model proposed for the determination of the gate bias voltage at ZTC point $\left(\mathrm{V}_{\mathrm{ZTC}}\right)$ using the first-order models of the drain current $\left(\mathrm{I}_{\mathrm{DS}}\right)$ characteristics as a function of the gate voltage $\left(\mathrm{V}_{\mathrm{GF}}\right)$, operating in the linear and saturation regimes.

To validate the model proposed results were compared with experimental data, and the analytical predictions are in very close agreement with experimental results in spite of the simplification used for the $V_{\text {ZTC }}$ model proposed.

Analysis was performed to study the impact on the $V_{\text {ZTC }}$ value with the change in the parameters used as reference, such as $\mathrm{N}_{\text {af }}$ and $\mathrm{t}_{\mathrm{oxf}}$. The maximum error observed for the PD devices is 3.1\% and $4.6 \%$ in the linear region and $3.5 \%$ and $7.2 \%$ in the saturation region, respectively. For FD devices the maximum error observed was $11 \%$ and $10 \%$ operating in the linear and $5.3 \%$ and $8.4 \%$ in the saturation regime.

In order to verify the stability of the ZTC point as a function of the mobility degradation (c), channel length $(L)$ and drain voltage $\left(V_{D S}\right)$, the proposed model was applied to the devices mentioned above. The $\mathrm{V}_{\mathrm{ZTC}}$ changes in the temperature range investigated showed a temperature mobility degradation dependence and are more pronounced in PD devices.

The $\mathrm{V}_{\mathrm{ZTC}}$ voltage for nMOS devices presented a lower value operating in the saturation region than in the linear region, and this difference becomes more pronounced to devices with smaller channel length for both devices, $\mathrm{n}$ and pMOS. Analyzing the $\mathrm{V}_{\mathrm{ZTC}}$ variation with drain voltage $\left(\mathrm{V}_{\mathrm{DS}}\right)$, showed a decrease in $\mathrm{V}_{\mathrm{ZTC}}$ value for higher $V_{D S}$, for both studied devices.

The model proposed results were evaluated using experimental data from other SOI MOSFET technologies. And also we have obtained for the GC SOI and GC-GAA-SOI technologies a very close agreement, operating in both regions, linear and saturation. 
Keywords: SOI technology. High temperature. Zero Temperature Coefficient. 


\section{LISTA DE FIGURAS}

Figura 1 - Representação esquemática do processo Smart-Cut.

Figura 2 - Transistor nMOSFET fabricado segundo tecnologia convencional (a) e SOI (b).

Figura 3 - Perfil do transistor nMOSFET SOI, ilustrando algumas das notações utilizadas.

Figura 4 - Diagrama de faixas de energia de dispositivos modo inversão canal tipo n: (A) MOS convencional, (B) SOI de camada espessa e (C) SOI de camada fina.

Figura 5 - Obtenção do fator de inclinação de sublimiar S em um transistor nMOS.

Figura 6 - Valores típicos de S encontrados em transistores MOS convencionais e SOI MOS totalmente depletados (FD).

Figura 7 - Extração de Vth usando o método de extrapolação linear para um dispositivo de canal curto operando na região linear.

Figura 8 - Extração de Vth usando o método de extrapolação linear para um dispositivo de canal curto operando na região de saturação.

Figura 9 - Extração de Vth usando o método de extrapolação linear para um dispositivo de canal curto operando na região de saturação.

Figura 10 - Curva característica IDS x VGF para um transistor SOI MOSFET parcialmente depletado para diversas temperaturas 56

Figura 11 - O ponto ZTC na curva IDS x VGS para temperaturas T1 e T2.

Figura 12 - Perfil do transistor nMOSFET SOI ilustrando as características dos dispositivos parcialmente e totalmente depletados 75

Figura 13 - Curvas $I_{D S} \times V_{G F}$ de medidas experimentais do transistor PD-SOI nMOS em diferentes temperaturas. 
Figura 14 - Curvas $I_{D S} \times V_{G F}$ de medidas experimentais do transistor FD-SOI nMOS em diferentes temperaturas.

Figura 15 - Curvas $I_{D S} \times V_{G F}$ de medidas experimentais do transistor PD-SOI pMOS em diferentes temperaturas.

Figura 16 - Curvas $I_{D S} \times V_{G F}$ de medidas experimentais do transistor FD-SOI pMOS em diferentes temperaturas.

Figura 17 - $V_{\text {ZTC }}$ obtido experimentalmente e através da equação (47) para dispositivos PD SOI nMOS

Figura 18 - $V_{\text {ZTC }}$ obtido experimentalmente e através da equação (47) para dispositivos FD SOI nMOS.

Figura 19 - $\mathrm{V}_{\text {ZTC }}$ obtido experimentalmente e através da equação (47) para dispositivos PD SOI pMOS.

Figura 20 - $\mathrm{V}_{\mathrm{ZTC}}$ obtido experimentalmente e através da equação (47) para dispositivos FD SOI pMOS.

Figura 21 - Curvas $\mathrm{I}_{\mathrm{DS}} \times \mathrm{V}_{\mathrm{GF}}$ de medidas experimentais do transistor PD-SOI nMOS em diferentes temperaturas.

Figura 22 - Curvas $I_{D S} \times V_{G F}$ de medidas experimentais do transistor FD-SOI nMOS em diferentes temperaturas.

Figura 23 - Curvas $I_{D S} \times V_{G F}$ de medidas experimentais do transistor PD-SOI pMOS em diferentes temperaturas

Figura 24 - Curvas $I_{D S} \times V_{G F}$ de medidas experimentais do transistor FD-SOI pMOS em diferentes temperaturas.

Figura 25 - $\mathrm{V}_{\mathrm{ZTC}}$ obtido experimentalmente e pelas equações (51),

(54) e (55) para dispositivo PD SOI nMOS.

Figura 26 - $\mathrm{V}_{\mathrm{ZTC}}$ obtido experimentalmente e pelas equações (51) ,

(54) e (55) para dispositivo FD SOI nMOS. 85

Figura 27 - $\mathrm{V}_{\text {ZTC }}$ obtido experimentalmente e pelas equações (51), (54) e (55) para dispositivo PD SOI pMOS. 86

Figura 28 - $\mathrm{V}_{\mathrm{ZTC}}$ obtido experimentalmente e pelas equações (51), (54) e (55) para dispositivo FD SOI pMOS. 86 


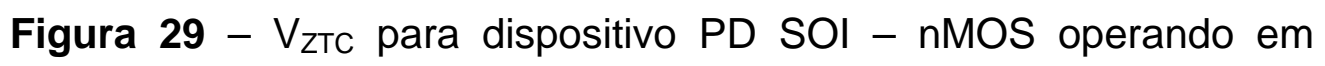
regime linear em função da temperatura para variações do fator $c$ entre 1 e 3

Figura 30 - V ZTC para dispositivo FD SOI - nMOS operando em regime linear em função da temperatura para variações do fator $c$ entre 1 e 3

Figura 31 - $\mathrm{V}_{\mathrm{ZTC}}(\mathrm{T} 1, \mathrm{~T} 2)$ para dispositivo PD SOI - nMOS operando em regime de saturação em função da temperatura para variação de $c$ entre 1 e 3

Figura 32 - $\mathrm{V}_{\mathrm{ZTC}}(\mathrm{T} 1, \mathrm{~T} 2)$ para dispositivo FD SOI - nMOS operando em regime de saturação em função da temperatura para variação de $c$ entre 1 e 3

Figura 33 - A variação de $\mathrm{V}_{\mathrm{ZTC}}$ com a temperatura (T2) em função do fator de degradação da mobilidade com a temperatura para dispositivos PD SOI nMOS nas regiões linear e de saturação.

Figura 34 - A variação de $V_{\text {ZTC }}$ com a temperatura (T2) em função do fator de degradação da mobilidade com a temperatura para dispositivos FD SOI nMOS nas regiões linear e de saturação. 94

Figura $35-\Delta \mathrm{V}_{\mathrm{ZTC}}$ para dispositivos PD SOI nMOS 95

Figura $36-\Delta \mathrm{V}_{\mathrm{ZTC}}$ para dispositivos FD SOI nMOS 95

Figura 37 - Tensão de polarização no ponto ZTC ( $\left.\mathrm{V}_{\text {ZTC }}\right)$ obtido experimentalmente e pelo modelo simples proposto para dispositivos PD nMOS SOI operando na região linear, para valores de comprimentos de canal (L) de 0,5 , 1, 5 e $10 \mu \mathrm{m}$ 99

Figura 38 - Tensão de polarização no ponto ZTC ( $\left.\mathrm{V}_{\mathrm{ZTC}}\right)$ obtido experimentalmente e pelo modelo simples proposto para dispositivos PD nMOS SOI operando na região de saturação, para valores de comprimentos de canal (L) de 0,5, 1, 5 e $10 \mu \mathrm{m}$. 100

Figura 39 - Tensão de polarização no ponto ZTC ( $\left.\mathrm{V}_{\text {ZTC }}\right)$ obtido experimentalmente e pelo modelo simples proposto para dispositivos PD pMOS SOI operando na região linear, para valores de comprimentos de canal (L) de 0,5, 1, 5 e $10 \mu \mathrm{m}$. 
Figura 40 - Tensão de polarização no ponto ZTC $\left(\mathrm{V}_{\mathrm{ZTC}}\right)$ obtido experimentalmente e pelo modelo simples proposto para dispositivos PD pMOS SOI operando na região de saturação, para valores de comprimentos de canal (L) de $0,5,5$ e $10 \mu \mathrm{m}$ 101

Figura 41 - Tensão de polarização no ponto ZTC ( $\left.\mathrm{V}_{\mathrm{ZTC}}\right)$ obtido experimentalmente em função do comprimento de canal para dispositivos PD SOI nMOS operando nas regiões linear $\left(V_{D S}=25 \mathrm{mV}\right)$ e de saturação $\left(V_{D S}=1.425 \mathrm{~V}\right)$, para valores de comprimento de canal. de $0,5,1,5$ e $10 \mu \mathrm{m}$.

Figura 42 - Tensão de polarização no ponto ZTC $\left(\mathrm{V}_{\mathrm{ZTC}}\right)$ obtido experimentalmente em função do comprimento de canal para dispositivos PD SOI pMOS operando na região linear $\left(\mathrm{V}_{D S}=25 \mathrm{mV}\right)$, para valores de comprimento de canal de 0,5, 1, 5 e $10 \mu \mathrm{m}$.

Figura 43 - Tensão de polarização no ponto ZTC ( $\left.V_{Z T C}\right)$ obtido experimentalmente em função do comprimento de canal para dispositivos PD SOI pMOS operando na região de saturação $\left(V_{D S}=\right.$ 1,425 V), para valores de comprimento de canal de 0,5 , 1, 5 e $10 \mu \mathrm{m}$ 103

Figura 44 - Tensão de polarização no ponto ZTC $\left(\mathrm{V}_{\mathrm{ZTC}}\right)$ obtido experimentalmente em função do comprimento de canal para dispositivos PD SOI pMOS operando na região de saturação $\left(\mathrm{V}_{\mathrm{DS}}=\right.$ $1,425 \mathrm{~V}$ ), para valores de comprimento de canal de 0,5 , 1, 5 e $10 \mu \mathrm{m}$. 105

Figura 45 - Dados experimentais: Mobilidade dos portadores no canal $\left(\mu_{n}\right)$ em função da tensão de dreno $\left(V_{D S}\right)$ para dispositivos PD SOI nMOS, para temperaturas de 300 a $573 \mathrm{~K}$. 106

Figura 46 - Variação da tensão de polarização no ponto ZTC ( $\left.V_{\text {ZTC }}\right)$ em função da tensão de dreno $\left(V_{D S}\right)$, obtida experimentalmente para dispositivos PD SOI nMOS com comprimentos de canal (L) de 0,5, 1, 5 e $10 \mu \mathrm{m}$. 106

Figura 47 - Variação da tensão de polarização no ponto ZTC ( $\left.V_{Z T C}\right)$ em função da tensão de dreno $\left(V_{D S}\right)$, obtida experimentalmente para dispositivos PD SOI pMOS com comprimentos de canal (L) de 0,5, 5 e $10 \mu \mathrm{m}$. 
Figura 48 - Perfis da estrutura GC GAA SOI: Vista em perspectiva (A) e Corte longitundinal 109

Figura 49: Curvas $I_{D S} \times V_{G F}$ de medidas experimentais do transistor GC SOI nMOS $\left(L_{L D} / L=0,25\right)$ em diferentes temperaturas para $V_{D S}=$ $100 \mathrm{mV}$.

Figura 50: Curvas $I_{D S} \times V_{G F}$ de medidas experimentais do transistor GC SOI nMOS $\left(L_{L D} / L=0,25\right)$ em diferentes temperaturas para $V_{D S}=$ $1,5 \mathrm{~V}$.

Figura 51: Curvas $I_{D S} \times V_{G F}$ de medidas experimentais do transistor GC SOI nMOS $\left(L_{L D} / L=0,5\right)$ em diferentes temperaturas para $V_{D S}=$ $100 \mathrm{mV}$.

Figura 52: Curvas $I_{D S} \times V_{G F}$ de medidas experimentais do transistor GC SOI nMOS $\left(L_{L D} / L=0,5\right)$ em diferentes temperaturas para $V_{D S}=$ $1,5 \mathrm{~V}$.

Figura 53: Tensão $V_{\text {ZTC }}$ obtido experimentalmente e pelo modelo proposto em função da temperatura para dispositivos SOI nMOS Convencionais, para as duas regiões de operação linear $\left(\mathrm{V}_{\mathrm{DS}}=100\right.$ $\mathrm{mV}$ ) e de saturação $\left(\mathrm{V}_{\mathrm{DS}}=1,5 \mathrm{~V}\right)$, comprimento de canal de $\mathrm{L}=4 \mu \mathrm{m}$.

Figura 54: Tensão $V_{\text {ZTC }}$ obtido experimentalmente e pelo modelo proposto em função da temperatura para dispositivos GC SOI nMOS, para as duas regiões de operação linear $\left(V_{D S}=100 \mathrm{mV}\right)$ e de saturação $\left(\mathrm{V}_{\mathrm{DS}}=1,5 \mathrm{~V}\right)$, para relação de comprimento de canal $\mathrm{L}_{\mathrm{LD}} / \mathrm{L}$ $=0,25$ ou $L_{H D}=3 \mu \mathrm{m}$.

Figura 55: Tensão $V_{\text {ZTC }}$ obtido experimentalmente e pelo modelo proposto em função da temperatura para dispositivos GC SOI nMOS, para as duas regiões de operação linear $\left(V_{D S}=100 \mathrm{mV}\right)$ e de saturação $\left(V_{D S}=1,5 \mathrm{~V}\right)$, para relação de comprimento de canal $L_{L D} / L$ $=0,5$ ou $\mathrm{L}_{\mathrm{HD}}=2 \mu \mathrm{m}$.

Figura 56: Tensão $\mathrm{V}_{\text {ZTC }}$ obtido experimentalmente e pelo modelo proposto em função da temperatura para dispositivos GC SOI nMOS, operando na região linear $\left(\mathrm{V}_{\mathrm{DS}}=100 \mathrm{mV}\right)$, para comprimento de canal $\mathrm{L}_{\mathrm{HD}}=0,7 \mu \mathrm{m}$. 
Figura 57: Tensão $\mathrm{V}_{\mathrm{ZTC}}$ obtido experimentalmente e pelo modelo proposto em função da temperatura para dispositivos GC SOI nMOS, operando na região de saturação $\left(\mathrm{V}_{\mathrm{DS}}=1,5 \mathrm{~V}\right)$, para comprimento de canal $\mathrm{L}_{H D}=1,5 \mu \mathrm{m}$

Figura 58: Tensão $V_{Z T C}$ em função do comprimento de canal da região altamente dopada $\left(\mathrm{L}_{\mathrm{HD}}\right)$ obtido experimentalmente, para dispositivos GC SOI nMOS operando na região linear $\left(V_{D S}=100 \mathrm{mV}\right)$

Figura 59: Tensão $V_{\text {ZTC }}$ em função do comprimento de canal da região altamente dopada $\left(\mathrm{L}_{\mathrm{HD}}\right)$ obtido experimentalmente, para dispositivos GC SOI nMOS operando na região de saturação $\left(V_{D S}=\right.$ $1,5 \mathrm{~V})$

Figura 60 - Curvas $I_{D S} \times V_{G F}$ de medidas experimentais do transistor GC-GAA SOI nMOS $\left(L_{L D} / L=0,17\right)$ em diferentes temperaturas.

Figura 61 - Curvas $I_{D S} \times V_{G F}$ de medidas experimentais do transistor GC-GAA SOI nMOS ( $\left.L_{L D} / L=0,33\right)$ em diferentes temperaturas.

Figura 62 - Curvas $I_{D S} \times V_{G F}$ de medidas experimentais do transistor GC-GAA SOI nMOS ( $\left.L_{L D} / L=0,67\right)$ em diferentes temperaturas.

Figura 63 - Tensão de polarização no ponto ZTC $\left(V_{Z T C}\right)$ obtido experimentalmente em função do comprimento de canal para dispositivos GC GAA SOI nMOS operando na região linear $\left(V_{D S}=50\right.$ $\mathrm{mV})$, para valores de relação de comprimento de canal $\left(L_{L D} / L\right)$ de $0,17,0,33$ e 0,67 .

Figura 64 - Tensão VZTC obtido pelo modelo proposto para dispositivos GC GAA SOI nMOS para uma relação de comprimento de canal $\left(L_{L D} / L\right)$ de 0,67 , operando na região linear $\left(V_{D S}=50 \mathrm{mV}\right)$, com valores do fator $\mathrm{c}$ de 1, 1,5, 2, 2,5 e 3

Figura 65: Variação do fator de degradação da mobilidade com a temperatura (Fator $\mathrm{C}$ ) 
Figura 66: Tensão de polarização no ponto ZTC $\left(\mathrm{V}_{\mathrm{ZTC}}\right)$ obtido experimentalmente em função da temperatura para dispositivos GC GAA SOI nMOS operando na região linear $\left(V_{D S}=50 \mathrm{mV}\right)$, para valores de relação de comprimento de canal $\left(L_{L D} / L\right)$ de $0,17,0,33$ e 0,67 .

Figura 67: Tensão de polarização no ponto ZTC $\left(\mathrm{V}_{\mathrm{ZTC}}\right)$ em função do comprimento de canal da região altamente dopada $\left(\mathrm{L}_{\mathrm{HD}}\right)$ obtido experimentalmente, para dispositivos GC GAA SOI nMOS operando na região linear $\left(V_{D S}=50 \mathrm{mV}\right)$.

Figura 68 -Dados experimentais: Variação da Tensão de limiar $\left(\mathrm{V}_{\mathrm{thF}}\right)$ em função do comprimento de canal da região altamente dopada (LHD) para dispositivos GC GAA SOI nMOS, para temperaturas de 300 a $573 \mathrm{~K}$.

Figura 69: Curvas $I_{D S} \times V_{G F}$ de medidas experimentais do transistor GC-GAA SOI nMOS $\left(L_{L D} / L=0,17\right)$ em diferentes temperaturas para $V_{D S}=1,5 \mathrm{~V}$

Figura 70: Curvas $I_{D S} \times V_{G F}$ de medidas experimentais do transistor GC-GAA SOI nMOS $\left(L_{L D} / L=0,33\right)$ em diferentes temperaturas para $\mathrm{V}_{\mathrm{DS}}=1,5 \mathrm{~V}$

Figura 71: Curvas $I_{D S} \times V_{G F}$ de medidas experimentais do transistor GC-GAA SOI nMOS $\left(L_{L D} / L=0,67\right)$ em diferentes temperaturas para $V_{D S}=1,5 \mathrm{~V}$

Figura 72: Tensão $V_{\mathrm{ZTC}}$ obtido experimentalmente e pelo modelo proposto em função da temperatura para dispositivos GC GAA SOI nMOS operando na região de saturação $\left(V_{D S}=1,5 \mathrm{~V}\right)$, para valores de relação de comprimento de canal ( $\left.\mathrm{L}_{\mathrm{LD}} / \mathrm{L}\right)$ de 0,17, 0,33 e 0,67.

Figura 73: Tensão $\mathrm{V}_{\mathrm{ZTC}}$ obtido pelo modelo proposto para dispositivos GC-GAA SOI nMOS para uma relação de comprimento de canal $\left(L_{L D} / L\right)$ de 0,67 , operando na região de saturação $\left(V_{D S}=1,5 \mathrm{~V}\right)$, com valores do fator $\mathrm{c}$ de $1,1,5,2,2,5$ e 3 .

Figura 74: Variação do fator de degradação da mobilidade com a temperatura (Fator C) para GC-GAA SOI em regime de saturação. 
Figura 75: Tensão de polarização no ponto ZTC $\left(\mathrm{V}_{\mathrm{ZTC}}\right)$ obtido experimentalmente em função da temperatura para dispositivos GCGAA SOI nMOS operando na região de saturação $\left(V_{D S}=1,5 \mathrm{~V}\right)$, para valores de relação de comprimento de canal $\left(L_{L D} / L\right)$ de $0,17,0,33$ e 0,67 .

Figura 76: Tensão de polarização no ponto ZTC $\left(V_{Z T C}\right)$ em função do comprimento de canal da região altamente dopada ( $\left.L_{H D}\right)$ obtido experimentalmente, para dispositivos GC-GAA SOI nMOS operando na região de saturação $\left(V_{D S}=1,5 \mathrm{~V}\right)$. 


\section{LISTA DE TABELAS}

Tabela 1 : Modos de operação SOI MOSFET dependendo do material de porta.

Tabela 2: Variação de $\mathrm{V}_{\text {ZTC }}$ para um intervalo de temperatura de $313 \mathrm{~K}$ a $573 \mathrm{~K}$

Tabela 3: Sensibilidade do método proposto através da análise da variação em $\Delta$ Vztc com uma variação de $10 \%$ nos valores de $N_{a}$ e $t_{\text {oxf }}$ para dispositivos PD SOI nMOS operando nos regimes linear e de saturação

Tabela 4: Sensibilidade do método proposto através da análise da variação em $\Delta$ Vztc com uma variação de $10 \%$ nos valores de $N_{a}$ e $t_{\text {oxf }}$ para dispositivos FD SOI nMOS operando nos regimes linear e de saturação

Tabela 5: Média aritmética do valor do fator c para cada comprimento de canal $L_{H D}$, para faixa de temperatura analisada, 300 a $573 \mathrm{~K}$. 


\section{LISTA DE SÍMBOLOS}
A, B
Termos da expressão de determinação de $\mathrm{V}_{\mathrm{ZTC}}$ na região de saturação do modelo CM
$A_{O s}, B_{O s}$ e $C_{O s}$
Parâmetros determinados no ponto ZTC de acordo com regime, linear ou de saturação (Modelo $\mathrm{V}_{\text {ZTC }}$ - Osman)
C Fator de degradação da mobilidade com a temperatura
$\mathrm{C}_{\mathrm{D}}$ Capacitância da região de depleção por unidade de área $\left[\mathrm{F} / \mathrm{cm}^{2}\right]$

$\mathrm{C}_{\mathrm{ox}}$
$\mathrm{C}_{\mathrm{oxb}}$
Capacitância do óxido de porta do transistor MOS convencional por unidade de área $\left[\mathrm{F} / \mathrm{cm}^{2}\right]$
Capacitância do óxido enterrado por unidade de área $\left[\mathrm{F} / \mathrm{cm}^{2}\right]$
$\mathrm{C}_{\mathrm{oxf}}$
Capacitância do óxido de porta do transistor SOI por unidade de área $\left[\mathrm{F} / \mathrm{cm}^{2}\right]$
$\mathrm{C}_{\mathrm{Si}} \quad$ Capacitância da camada de silício por unidade de área $\left[\mathrm{F} / \mathrm{cm}^{2}\right]$
E Nível energético do extremo inferior da faixa de Condução [eV]
$\mathrm{E}_{\mathrm{F}} \quad$ Nível de Fermi [eV]
Ei Nível de Fermi intrínseco [eV]
$E_{V} \quad$ Nível energético do extremo superior da faixa de Valência [eV]
$g_{\mathrm{m}} \quad$ Transcondutância, relação entre a variação na corrente $\mathrm{I}_{\mathrm{DS}}$ e a variação em $V_{G F}$ que a provoca $[A / V]$
$g_{\mathrm{m} \max } \quad$ Transcondutância máxima [A/V]
ID $\quad$ Corrente de Dreno $[A]$
IDS $\quad$ Corrente entre Dreno e Fonte $[A]$
I I Corrente entre Dreno e Fonte com tensão de polarização no ponto ZTC [A]
k Constante de Boltzmann $\left[1,38066 \times 10^{-23} \mathrm{~J} / \mathrm{K}\right]$ 


\begin{tabular}{|c|c|}
\hline L & Comprimento de canal do transistor $[\mu \mathrm{m}]$ \\
\hline $\mathrm{L}_{\mathrm{LD}}$ & $\begin{array}{l}\text { Comprimento de canal do transistor GC-SOI da região } \\
\text { menos dopada }[\mu \mathrm{m}]\end{array}$ \\
\hline $\mathrm{L}_{H D}$ & $\begin{array}{l}\text { Comprimento de canal do transistor GC-SOI da região } \\
\text { mais dopada }[\mu \mathrm{m}]\end{array}$ \\
\hline $\mathrm{n}$ & Fator de efeito de corpo do transistor SOI \\
\hline$n_{i}$ & Concentração intrínseca de dopantes $\left[\mathrm{cm}^{-3}\right]$ \\
\hline $\mathrm{N}_{\mathrm{a}}$ & $\begin{array}{l}\text { Concentração de impurezas aceitadoras do semicondutor } \\
\text { do transistor MOS convencional }\left[\mathrm{cm}^{-3}\right]\end{array}$ \\
\hline $\mathrm{N}_{\mathrm{af}}$ & $\begin{array}{l}\text { Concentração de impurezas aceitadoras da camada de } \\
\text { silício do transistor SOI }\left[\mathrm{cm}^{-3}\right]\end{array}$ \\
\hline q & Carga elementar do elétron $[\mathrm{C}]$ \\
\hline$Q_{\text {depl }}$ & $\begin{array}{l}\text { Densidade de carga de depleção na camada de silício } \\
{\left[{\left.\mathrm{C} / \mathrm{cm}^{2}\right]}\right.}\end{array}$ \\
\hline $\mathrm{Q}_{\text {inv1 }}$ & $\begin{array}{l}\text { Densidade de carga de inversão na primeira interface } \\
{\left[\mathrm{C} / \mathrm{cm}^{2}\right]}\end{array}$ \\
\hline$Q_{o x}$ & 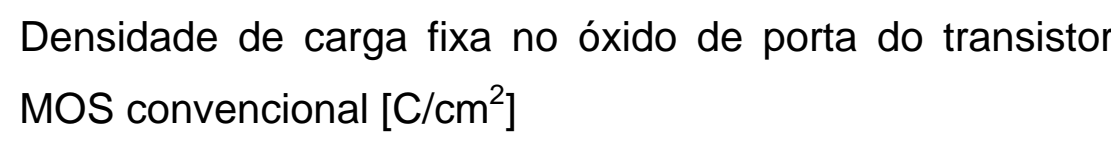 \\
\hline Qoxf & 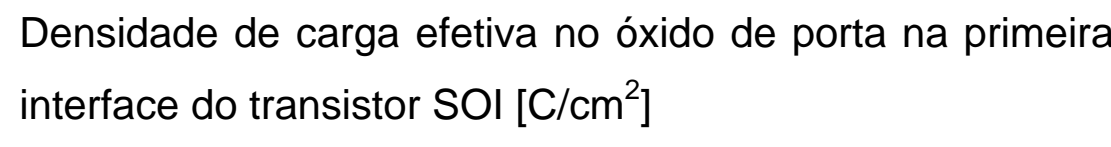 \\
\hline Qoxb & $\begin{array}{l}\text { Densidade de carga efetiva no óxido enterrado na } \\
\text { segunda interface do transistor } \mathrm{SOI}\left[\mathrm{C} / \mathrm{cm}^{2}\right]\end{array}$ \\
\hline $\mathrm{Q}_{\mathrm{s} 2}$ & $\begin{array}{l}\text { Densidade de carga de acumulação ou inversão na } \\
\text { segunda interface do transistor } \mathrm{SOI}\left[\mathrm{C} / \mathrm{cm}^{2}\right]\end{array}$ \\
\hline S & Inclinação de Sublimiar [mV/dec.] \\
\hline $\mathrm{T}$ & Temperatura absoluta $[\mathrm{K}]$ \\
\hline $\mathrm{T}_{0}$ & Temperatura ambiente em valor absoluto $(\mathrm{K})$ \\
\hline$t_{\mathrm{ox}}$ & Espessura do óxido de porta [nm] \\
\hline$t_{\text {oxb }}$ & Espessura do óxido enterrado [nm] \\
\hline$t_{\text {oxf }}$ & Espessura do óxido de porta [nm] \\
\hline$t_{\mathrm{Si}}$ & Espessura do filme de silício [nm] \\
\hline$V_{D S}$ & Tensão entre Dreno e Fonte [V] \\
\hline
\end{tabular}




\begin{tabular}{|c|c|}
\hline $\mathrm{V}_{\mathrm{FB}}$ & $\begin{array}{l}\text { Tensão de Faixa Plana da primeira interface induzida pela } \\
\text { porta do transistor SOI }[\mathrm{V}]\end{array}$ \\
\hline $\mathrm{V}_{\mathrm{GF}}$ & Tensão de porta do transistor SOI [V] \\
\hline$V_{G B}$ & Tensão de substrato do transistor SOI [V] \\
\hline $\mathrm{V}_{\mathrm{GB}, \mathrm{acC}}$ & $\begin{array}{l}\text { Tensão de substrato com a segunda interface em } \\
\text { acumulação [V] }\end{array}$ \\
\hline$V_{G B}$, inv & $\begin{array}{l}\text { Tensão de substrato com a segunda interface em inversão } \\
\text { [V] }\end{array}$ \\
\hline$V_{G S}$ & Tensão de porta do transistor MOS convencional [V] \\
\hline $\mathrm{V}_{\text {th }}$ & Tensão de limiar do transistor MOSFET Convencional [V] \\
\hline $\mathrm{V}_{\text {th1 }}, \mathrm{V}_{\text {th2 }}$ & $\begin{array}{l}\text { Tensão de limiar do transistor MOSFET na temperatura } \\
\text { igual a T1 e T2 [V] }\end{array}$ \\
\hline$V_{\text {thF }}$ & $\begin{array}{l}\text { Tensão de limiar do transistor SOI MOSFET relativo a } \\
\text { primeira interface }[\mathrm{V}]\end{array}$ \\
\hline $\mathrm{V}_{\mathrm{thF}}$, depl2 & $\begin{array}{l}\text { Tensão de limiar do transistor SOI MOSFET relativo a } \\
\text { primeira interface com a segunda interface em depleção } \\
\text { [V] }\end{array}$ \\
\hline $\mathrm{V}_{\mathrm{ZTC}}$ & Tensão de polarização do transistor no ponto ZTC [V] \\
\hline$V_{\text {ZTC LIN }}$ & $\begin{array}{l}\text { Tensão de polarização do transistor no ponto ZTC [V] na } \\
\text { região linear de operação }\end{array}$ \\
\hline V ZTC SAT & $\begin{array}{l}\text { Tensão de polarização do transistor no ponto ZTC [V] na } \\
\text { região de saturação de operação }\end{array}$ \\
\hline$V_{\text {ZTC }}(T 1, T 2)$ LIN & $\begin{array}{l}\text { Tensão de polarização do transistor no ponto ZTC [V] na } \\
\text { região linear de operação }\end{array}$ \\
\hline$V_{Z T C}(T 1, T 2)_{S A T}$ & $\begin{array}{l}\text { Tensão de polarização do transistor no ponto ZTC [V] na } \\
\text { região de saturação de operação }\end{array}$ \\
\hline W & Largura de canal do transistor $[\mu \mathrm{m}]$ \\
\hline Xdmáx & Profundidade da região de depleção máxima $[\mu \mathrm{m}]$ \\
\hline$\alpha$ & $\begin{array}{l}\text { Parâmetro resultante da associação das capacitâncias do } \\
\text { transistor MOSFET }\end{array}$ \\
\hline$\beta_{0}$ & $\begin{array}{l}\text { Parâmetro empírico com valor entre } 0.001 \text { and } 0.004 \\
\mathrm{~mm} / \mathrm{V}\end{array}$ \\
\hline
\end{tabular}


Constante dielétrica

$\varepsilon_{\mathrm{OX}}$

ESi

ESiO2

$\phi$

$\phi_{\mathrm{F}}$

$\phi_{\mathrm{Ff}}$

$\phi_{\mathrm{M}}$

$\phi_{\mathrm{MS}}$

$\phi_{\mathrm{MS} 1}$

$\phi_{\mathrm{MS} 2}$

$\phi_{\mathrm{SB}}$

$\phi_{\text {SF }}$

$\phi_{\text {Si }}$

$\phi_{\text {SUB }}$

$\delta$

$\gamma$

$\theta$

$\rho$

$\mu_{0}$

Permissividade do óxido de porta

Permissividade do silício $\left[1,06 \times 10^{-12} \mathrm{~F} / \mathrm{cm}\right]$

Permissividade do óxido de silício $\left[3,45 \times 10^{-13} \mathrm{~F} / \mathrm{cm}\right]$

Potencial [V]

Potencial de Fermi [V]

Potencial de Fermi $[V]$ do filme de silício do transistor SOI

[V]

Função trabalho do material de porta [V]

Diferença de função trabalho entre metal de porta e semicondutor [V]

Diferença de função trabalho entre metal de porta e a camada de silício [V]

Diferença de função trabalho entre o substrato e a camada de silício [V]

Potencial de superfície da segunda interface [V]

Potencial de superfície da primeira interface [V]

Função trabalho do canal de silício [V]

Potencial de superfície da terceira interface ou do substrato [V]

Representa a o fator de contribuição da tensão de dreno $\left(V_{D S}\right)$ sobre a largura da camada de depleção (Modelo $\mathrm{V}_{\mathrm{ZTC}}-$ Prijic)

Constante de Efeito de Corpo para transistor MOS convencional $\left[\mathrm{V}^{1 / 2}\right]$

Fator de Efeito da degradação da mobilidade por efeito de campo elétrico transversal

Parâmetro de contribuição da tensão de dreno $\left(V_{D S}\right)$ na corrente $I_{D S}$ (Modelo $V_{Z T C}-$ Prijic)

Mobilidade de baixo campo elétrico dos elétrons na camada de silício $\left[\mathrm{cm}^{2} / \mathrm{V} \cdot \mathrm{s}\right]$ 
$\mu_{n}, \mu_{\text {eff }}$

$\mu_{\text {sat }}$
Mobilidade efetiva dos elétrons na camada de silício $\left[\mathrm{cm}^{2} / \mathrm{N} \cdot \mathrm{s}\right]$

Mobilidade dos elétrons na camada de silício com o transistor operando na região de saturação $\left[\mathrm{cm}^{2} / \mathrm{V} \cdot \mathrm{s}\right]$ 


\title{
SUMÁRIO
}

\begin{abstract}
Resumo
Lista de Figuras

Lista de Tabelas

Lista de Símbolos
\end{abstract}

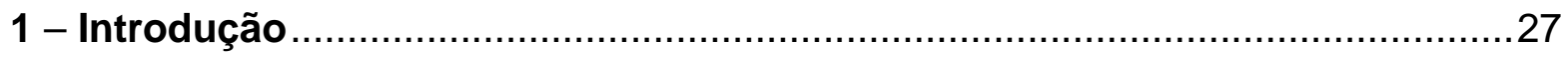

1.1 - Objetivos do trabalho e apresentação ......................................29

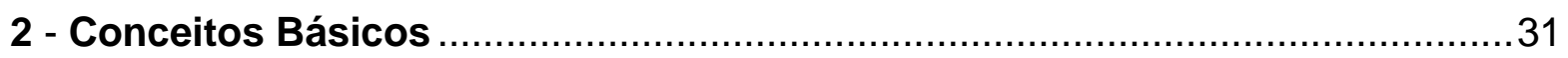

2.1 - Tecnologia de fabricação das lâminas SOI .......................................31

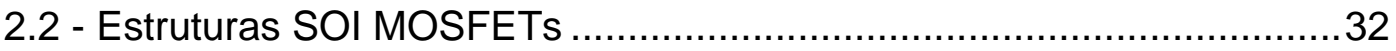

2.3 - Distinção entre transistores SOI ......................................................33

2.4 - Modo de Operação.................................................................. 37

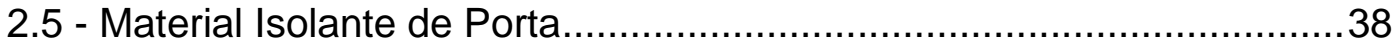

2.6 - Parâmetros Elétricos Básicos ........................................................39

2.6.1 - Tensão de Limiar ............................................................... 39

2.6.2 - Corrente de Dreno e Inclinação de Sublimiar.........................41

2.6.3 - Mobilidade dos portadores .......................................... 45

2.7 Métodos de Extração de Parâmetros ...............................................46

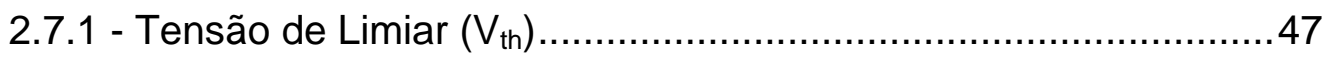

2.7.2 - Mobilidade Efetiva no Canal do Transistor...............................50

2.8 Aplicações em Altas Temperaturas e a Tecnologia SOI.......................52

3 - Ponto Invariante com a Temperatura ZTC ("Zero Temperature

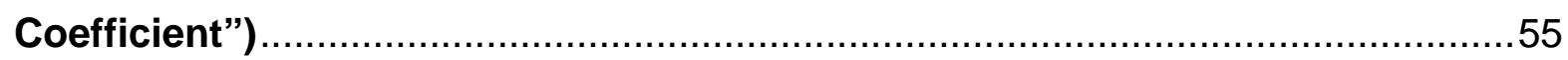

3.1 - Importância da determinação do ponto invariante com a

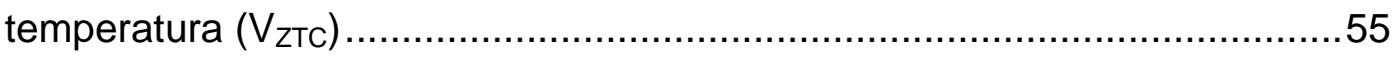


3.2 - Definição Ponto Invariante com a Temperatura (ZTC)

3.3 - Modelos sobre o Ponto Invariante com a Temperatura(ZTC) . .59

3.3.1 - Modelo $\mathrm{V}_{\text {ZTC }}$ para transistores CMOS convencionais Prijic .59

3.3.2 - Modelo $V_{\text {ZTC }}$ para MOS Convencionais - Shoucair 61

3.3.3 - Modelo $V_{\text {ZTC }}$ para SOI MOSFETs Parcialmente Depletados - Osman.

\section{4 - Proposta de um Modelo Analítico Simples para determinação da} tensão de polarização no ponto invariante com a temperatura ( $\left.\mathrm{V}_{\mathrm{ZTC}}\right)$

4.1 - Polarização no ponto invariante com a temperatura $\left(\mathrm{V}_{\mathrm{ZTC}}\right)$.

4.1.1 - Modelo Camillo-Martino (Modelo CM): Modelo de $\mathrm{V}_{\text {ZTC }}$ na

Região Linear 68

4.1.2 - Modelo Camillo-Martino (Modelo CM): Modelo de $\mathrm{V}_{\text {ZTC }}$ na

Região de Saturação. .72

4.2 - Validação do Modelo CM .74

4.2.1 - Características dos Dispositivos 74

4.2.2 - Resultados experimentais e modelo CM proposto para dispositivos SOI Convencionais .75

4.2.2.1 - Região Linear de Operação .76

4.2.2.2 - Região de Saturação de Operação. 82

5 - Estudo da estabilidade do Ponto Invariante com a Temperatura (ZTC).

5.1 - Influência do fator de degradação da mobilidade com a temperatura ( $c$ ) no ponto ZTC.

5.1.1 - Características físicas dos dispositivos .88

5.1 .2 - Influência do fator de degradação da mobilidade. .88

5.1.3 - Polarização na Região Linear .88

5.1.4 - Polarização na Região de Saturação 91

5.1.5 - Sensibilidade do Método Proposto 94 
5.2 - Influência da dependência da estabilidade do ponto ZTC com o

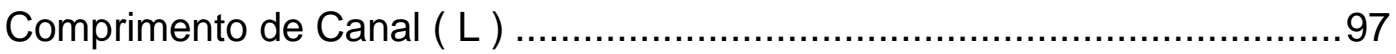

5.2.1 - Características físicas dos dispositivos ..................................97

5.2 .2 - Resultados e Discussão ................................................... 98

5.3 - Influência da dependência da estabilidade do ponto ZTC com a

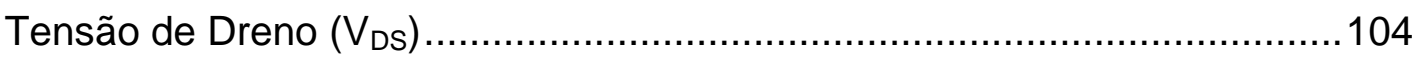

5.3.1 - Características físicas dos dispositivos................................... 104

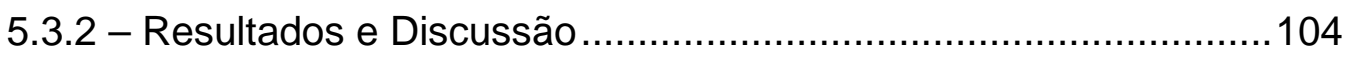

5.4 - Aplicação do modelo CM em outras tecnologias................................108

5.4.1 - Analise do modelo proposto em dispositivos GC SOI................109

5.4.1.1 - Características dos Dispositivos................................109

5.4.1.2 - Resultados e Discussão .........................................110

5.4.1.2.1 - Ocorrência do Ponto Invariante com a

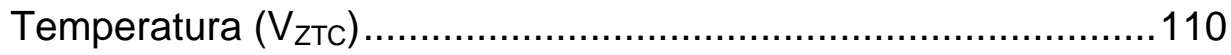

5.4.1.2.2 - V ZTC: Dados Experimentais e Modelo CM .................113

5.4.1.2.3 - Variação de $\mathrm{V}_{\text {ZTC }}$ com comprimento de canal (L)

5.4.2 - Analise do modelo CM proposto em dispositivos GC GAA SOI

5.4.2.1 - Características dos Dispositivos

5.4.2.2 - Resultados e Discussão 119

5.4.2.2.1 - Região Linear de Operação.....................................119

5.4.2.2.2 - Região de Saturação .125

6 - Conclusões

7 - Referências Bibliográficas 135

Apêndice A - Publicações geradas durante o trabalho . 141 


\section{Introdução}

Com o escalamento das dimensões, os dispositivos com tecnologia MOS (Metal Oxide Semiconductor), utilizados na produção de circuitos integrados digitais em grande escala de integração (GSI - "Giga Scale Integration"), apresentam um processo de fabricação cada vez mais complexo para contornar os inúmeros problemas decorrentes dos efeitos de canal curto provocados pela tendência de redução.

A tecnologia SOI ("Silicon On Insulator") ${ }^{1,2}$ inicialmente ficou restrita a poucas áreas de aplicação, como ambientes em condições severas, sujeitos à radiação e altas temperaturas, e aplicações de uso militar. A partir dos anos 80 , muitos trabalhos foram desenvolvidos nas áreas de materiais $\mathrm{e}$ dispositivos da tecnologia SOI, que deixou de ser apenas uma alternativa para ser uma realidade frente a tecnologia MOS convencional ("bulk").

Os circuitos implementados em lâminas SOI tornaram possível a fabricação de dispositivos com algumas características elétricas quase ideais, principalmente pela completa isolação dielétrica intrínseca entre os mesmos. O dióxido de silício é o isolante mais utilizado, mas existem outros materiais também estudados, como nitreto de Silício $^{3}$ e oxinitretos ${ }^{4}$.

O limite de operação das tecnologias de fabricação de circuitos integrados foi expandido nos últimos anos para temperaturas mais altas (até $300^{\circ} \mathrm{C}$ ) em áreas como o monitoramento e o controle de motores a jato, circuitos de controle na indústria automobilística, indústria de energia nuclear e aplicações espaciais ${ }^{5,6,7}$. O uso de dispositivos CMOS fabricados com a tecnologia MOS convencional é limitado pelo efeito do tiristor parasitário ("latch-up"), resultante da corrente de fuga através das junções entre as cavidades da estrutura CMOS, que se torna mais significativa em alta temperatura. A estrutura SOI CMOS é uma alternativa apropriada por causa 
de sua quase perfeita isolação dos transistores nMOS e pMOS, o que propicia a eliminação das cavidades na estrutura CMOS, resultando na operação do dispositivo livre do efeito tiristor parasitário ${ }^{5,8}$.

$\mathrm{Na}$ evolução da tecnologia SOI, a espessura da camada de silício sobre o isolante vem diminuindo e atingindo valores inferiores a $100 \mathrm{~nm}$, os chamados dispositivos SOI camada fina ("thin film SOI MOSFET"), os quais operando no modo totalmente depletado ("fully depleted"), apresentam as melhores vantagens em relação à tecnologia MOS convencional ${ }^{9}$. Dentre estas vantagens, citamos algumas que apresentam grande importância, como o aumento da mobilidade ${ }^{10}$, da transcondutância e da densidade de integração ${ }^{2}$, a diminuição das capacitâncias das regiões de Fonte e Dreno ${ }^{11}$, dos efeitos de canal curto $^{12}$ e da sensibilidade com a variação da temperatura ${ }^{13}$.

O funcionamento dos transistores MOS é fortemente dependente da temperatura ${ }^{14,15}$. Entre os parâmetros responsáveis por esta dependência os mais importantes são a mobilidade efetiva dos portadores $\left(\mu_{\text {eff }}\right)$ e o potencial de Fermi $\left(\phi_{F}\right)$, vindo este último a influênciar na tensão de limiar $V_{\text {th }}{ }^{14}$. Nos últimos anos, significativos progressos foram realizados nos processos de caracterização do comportamento elétrico de transistores na tecnologia CMOS, operando sobre uma larga faixa de temperatura ${ }^{16,17,18,19,20}$. No caso de circuitos projetados para aplicações em temperatura variável, o desejável é que operem em um ponto de polarização no qual o valor da corrente de dreno ( $\left.I_{D S}\right)$ não apresente variação, ou que seja muito pequena, com a mudança de temperatura. Este critério garante que os parâmetros dos blocos básicos de circuitos, como amplificadores e comparadores, sejam menos afetados no processo de casamento das características dos transistores que os compõem ${ }^{21}$.

Este ponto de polarização, muito importante para a estabilidade de um circuito operando sobre uma larga faixa de temperatura, é conhecido 
como Ponto Invariante com a Temperatura (ZTC - "Zero Temperature Coefficient"). Esse tema tem sido alvo de muitos trabalhos em dispositivos MOS convencionais ${ }^{16,22}$ e dispositivos SOI parcialmente depletados (PD) ${ }^{21}$, considerando a dependência da tensão de limiar $\left(\bigvee_{\text {thF }}\right)$ e da mobilidade $\left(\mu_{n}\right)$ com a temperatura ${ }^{16}$, incluindo o efeito de corpo $(n)^{22}$ e também a degradação da mobilidade com o campo elétrico transversal $(\theta)^{21}$.

\subsection{Objetivos do trabalho e apresentação}

O objetivo deste trabalho é o estudo do ponto ZTC em dispositivos SOI $\mathrm{n}$ e pMOSFETs, funcionando em regime parcialmente (PD-SOI) e totalmente (FD-SOI) depletados. O estudo é realizado a partir de um modelo analítico proposto (modelo CM: Camillo-Martino), e através de dados experimentais. Análise deste comportamento em diferentes tecnologias SOI será realizada teórica e experimentalmente.

Este trabalho encontra-se dividido em 6 capítulos, os quais estão a seguir:

No Capítulo 2 é apresentada uma revisão bibliográfica dos aspectos principais da tecnologia e funcionamento dos transistores SOI MOSFET operando em modo enriquecimento, abordando em detalhes os modelos analíticos, para permitir que eventuais simplificações sejam feitas, sem prejudicar os resultados obtidos no comportamento básico destes dispositivos.

No Capítulo 3 é apresentada uma revisão bibliográfica sobre ponto invariante com a temperatura (ZTC), a importância da polarização dos dispositivos no ponto ZTC através dos modelos existentes na literatura para estudo do seu comportamento nos regimes de triodo e saturação. 
No Capítulo 4 é apresentada uma proposta de um modelo analítico simples (modelo CM), para estudo dos aspectos do comportamento da tensão de porta $\left(\mathrm{V}_{\mathrm{GS}}\right)$ polarizada no ponto invariante com a temperatura $\left(\mathrm{V}_{\mathrm{ZTC}}\right)$, funcionando nas regiões linear e de saturação. A validação do modelo analítico simples proposto é realizada através de comparação entre os resultados de simulações do modelo e dados experimentais.

No Capítulo 5 são apresentados estudos da estabilidade do ponto ZTC em função da variação da degradação da mobilidade, do comprimento de canal $(L)$ e da tensão de dreno $\left(V_{D S}\right)$, através do modelo $C M$ proposto no capítulo 4, para dispositivos SOI MOSFETs convencionais operando em regime totalmente (FD) e parcialmente (PD) depletados. São apresentados também resultados do modelo $\mathrm{CM}$ proposto aplicado em dispositivos de tecnologia GC SOI e GC-GAA SOI.

No Capítulo 6 são apresentadas as conclusões das análises dos resultados obtidos pelo modelo $\mathrm{CM}$ proposto, para a tensão de porta do transistor polarizada no ponto invariante com a temperatura ( $\left.\mathrm{V}_{\mathrm{ZTC}}\right)$, nas regiões de polarização linear e saturação, para as tecnologias estudadas. 


\section{Conceitos Fundamentais}

Neste capítulo é apresentada uma revisão bibliográfica dos aspectos principais da tecnologia e funcionamento dos transistores SOI MOSFET operando em modo enriquecimento, abordando a influência de alguns parâmetros importantes na sua caracterização elétrica e nos modelos analíticos, para permitir que eventuais simplificações sejam realizadas, e deste modo não prejudiquem os resultados obtidos no comportamento básico destes dispositivos.

\subsection{Tecnologia de fabricação das lâminas SOI}

Entre os grandes fatores que contribuíram para uma maior divulgação e uso da tecnologia SOI, destaca-se a alta evolução dos processos de obtenção de suas lâminas ${ }^{23}$ que permitiu que esta se tornasse uma real alternativa. Atualmente, três processos apresentam viabilidade em termos de qualidade e de preço da lâmina: SIMOX ${ }^{24}$ baseada na técnica de implantação de íons de oxigênio, $\mathrm{BESO}^{25}$ baseada na técnica de união de lâminas e a mais recente SmartCut ${ }^{26}$, Figura 1.

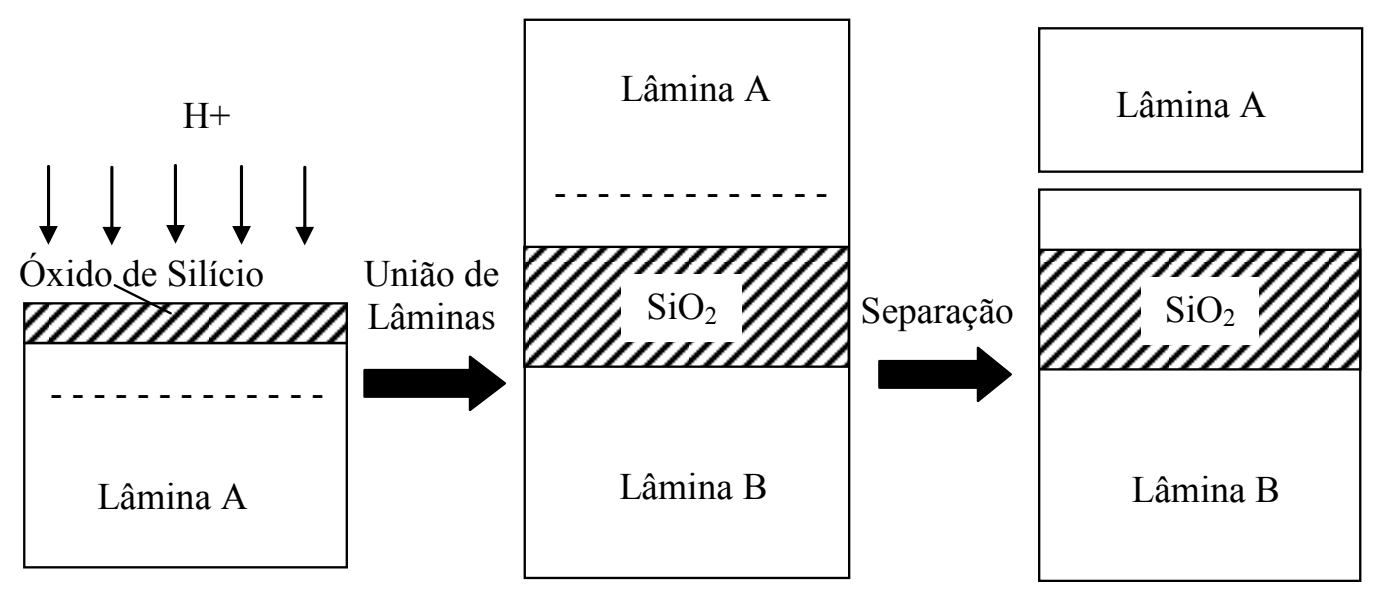

Figura 1- Representação esquemática do processo Smart-Cut. 
As maiores vantagens do processo Smart-Cut são o uso de um implantador iônico convencional e o aproveitamento da parcela restante da lâmina implantada para outro processamento posterior.

\subsection{Estruturas SOI MOSFETs}

A maioria dos dispositivos fabricados com tecnologia microeletrônica utiliza somente uma fina camada próxima a superfície da lâmina de semicondutor. Como exemplo, em dispositivos MOS convencionais, Figura $2 \mathrm{a}$, na realidade somente os primeiros 100 nanômetros da área superior da lâmina são usados para o fluxo de elétrons. O restante das centenas de micrômetros em uma lâmina espessa serve quase que exclusivamente para suporte mecânico aos dispositivos. A interação elétrica entre os dispositivos e o substrato introduz alguns efeitos parasitários prejudiciais, e além disso a condução elétrica que ocorre através do substrato torna difícil a total isolação dielétrica entre os dispositivos fisicamente próximos. Como conseqüência, os dispositivos parasitários podem tornar-se ativos, e com a redução das dimensões, seus efeitos tornam-se ainda mais pronunciados.

A idéia fundamental da tecnologia de fabricação de circuitos integrados em lâminas de Silício Sobre Isolante (SOI - "Silicon-OnInsulator"), Figura 2b, consiste em separar a região ativa da lâmina, onde os transistores são processados, do substrato de silício, pelo posicionamento de um material isolante entre ambos, de modo a eliminar ou reduzir os efeitos parasitários indesejáveis. Inicialmente o material isolante era a Safira e devido ao seu alto custo de processamento, foi substituída pelo óxido de silício que atualmente é utilizado. 


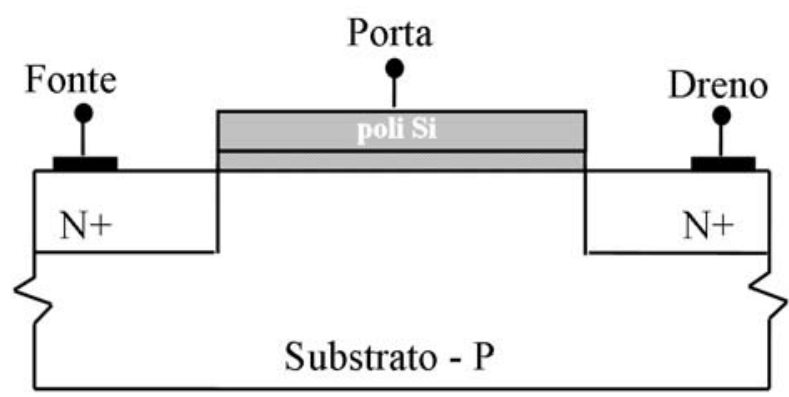

a)

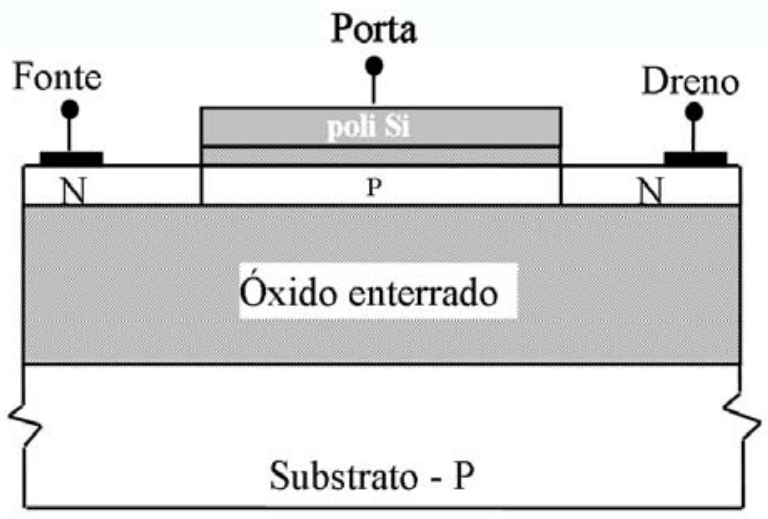

b)

Figura 2 - Transistor nMOSFET fabricado segundo tecnologia convencional (a) e SOI (b).

\subsection{Distinção entre transistores SOI}

A Figura 3 mostra o perfil de um transistor SOI MOSFET ilustrando algumas notações utilizadas no texto. Existem três tipos de dispositivos dependendo da espessura do filme de silício e da concentração de dopantes do canal ( $\mathrm{N}_{\mathrm{af}}$ ): dispositivo de camada espessa ("thick-Film"), dispositivo de camada fina ("thin-Film") e dispositivo de camada média ("medium thickness"), que pode exibir tanto um comportamento como o de camada fina quanto o de camada espessa, dependendo da polarização do substrato $\mathrm{V}_{\mathrm{GB}}$. 


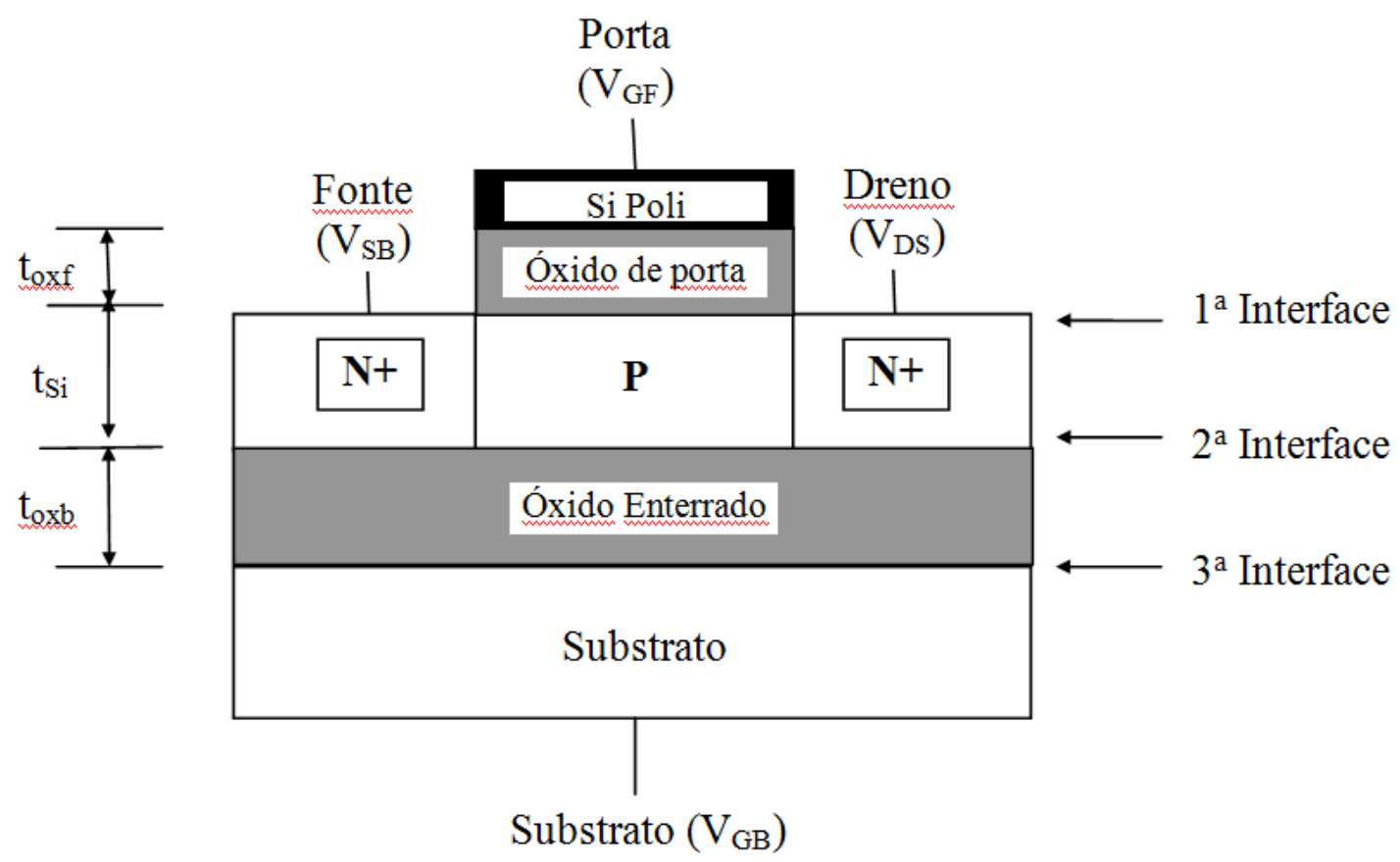

Figura 3 - Perfil do transistor nMOSFET SOI, ilustrando algumas das notações utilizadas.

Na Figura 4 são apresentados os diagramas das faixas de energia para dispositivos de canal $\mathrm{n}$ em modo inversão com tecnologia convencional ("bulk"), SOI de camada espessa e SOI de camada fina. Os níveis de energia de condução, Fermi e de valência são respectivamente, $E_{C}, E_{F}$ e $E_{V}$. Em um dispositivo convencional, Figura 4A, a região de depleção estende-se a partir da interface de $\mathrm{SiO}_{2}-\mathrm{Si}$ até a largura de depleção máxima, $\mathrm{x}_{\mathrm{dmáx}}$, que é classicamente dada por:

$$
\mathrm{x}_{\text {dmáx }}=\sqrt{\frac{4 \cdot \varepsilon_{\mathrm{Si}} \cdot \phi_{\mathrm{F}}}{\mathrm{q} \cdot \mathrm{N}_{\mathrm{a}}}}
$$




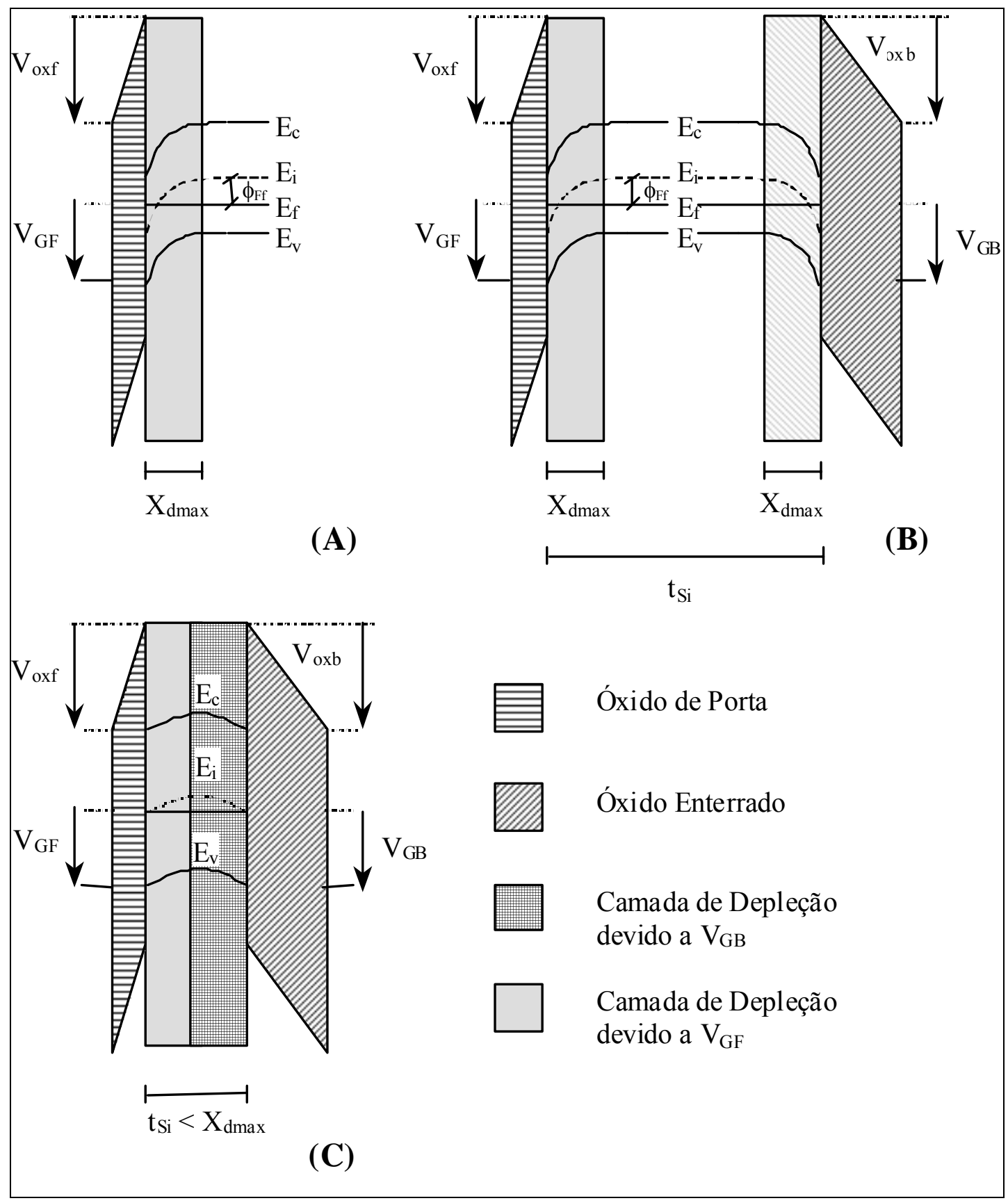

Figura 4 - Diagrama de faixas de energia de dispositivos modo inversão canal tipo n: (A) MOS convencional, (B) SOI de camada espessa e (C) SOI de camada fina.

onde $\varepsilon_{\mathrm{Si}}$ é a permissividade do silício, q é a carga elementar do elétron, $\mathrm{N}_{\mathrm{af}}$ é a concentração de dopantes da camada de Si e $\phi_{\mathrm{Ff}}$ é o potencial de Fermi dado por: 


$$
\phi_{\mathrm{Ff}}=\frac{\mathrm{kT}}{\mathrm{q}} \ln \left(\frac{\mathrm{N}_{\mathrm{af}}}{\mathrm{n}_{\mathrm{i}}}\right)
$$

e $n_{i}$ é a concentração intrínseca em função da temperatura $T$ dada pela expressão:

$$
\mathrm{n}_{\mathrm{i}}=3.910^{16} \mathrm{~T}^{3 / 2} \mathrm{e}^{-(\mathrm{Eg} / 2 \mathrm{kT})}
$$

Nos dispositivos SOI de camada espessa, Figura 4B, a espessura da camada de silício $\left(t_{S_{i}}\right)$ é maior que o dobro da largura máxima da região de depleção $\left(t_{S_{i}}>2 . x_{d m a ́ x}\right)$. Neste caso, não há interação entre as regiões de depleção originadas na primeira e segunda interfaces, pois existe uma região neutra do filme de silício entre as regiões de depleção. Deste modo o canal do transistor encontra-se parcialmente depletado ("Partial Depleted" PD), e quando a região neutra de silício, denominada "corpo", é conectada a um contato ôhmico, as características do dispositivo serão exatamente iguais ao dispositivo MOS convencional. Entretanto, se o "corpo" fica eletricamente flutuante, o dispositivo se comportará como um MOS convencional, porém com aparecimento notável de dois efeitos parasitários: o primeiro é chamado efeito de elevação abrupta de corrente ("Kink Effect") ${ }^{23}$, o segundo é o aumento do efeito bipolar parasitário (NPN) entre as regiões de fonte, canal e dreno ${ }^{23}$.

Nos dispositivos SOI de camada fina, Figura 4C, onde a espessura da camada de silício é menor que a largura máxima da região de depleção $\left(t_{S i}\right.$ $<X_{\text {dmáx }}$ ), o filme de silício fica totalmente depletado ("Fully Depleted" - FD) se a tensão aplicada no eletrodo de porta $\left(\mathrm{V}_{\mathrm{GF}}\right)$ atingir a tensão de limiar $\left(\mathrm{V}_{\mathrm{thF}}\right)$ independentemente da tensão aplicada no substrato. Os dispositivos SOI totalmente depletados (FD-SOI), exibem as propriedades mais atrativas entre todos os tipos de dispositivos SOI, quando apresentam a segunda interface depletada. Algumas das propriedades são a supressão do efeito de elevação abrupta de corrente, redução do campo elétrico horizontal ${ }^{27}$, alta 
transcondutância ${ }^{23}$, menor ocorrência de efeitos de canal curto ${ }^{13}$ e quase ideal taxa de variação de corrente na região de sublimiar (S - "Slope").

Os dispositivos SOI de camada de silício de espessura média ("medium thickness") são um caso intermediário entre dispositivo de camada espessa e camada fina, e são obtidos no caso onde $x_{d m a ́ x}<t_{S i}<2$. $x_{d m a ́ x}$. Pode ocorrer ou não a interação entre as duas regiões de depleção da primeira e segunda interfaces, apresentando comportamento elétrico de parcialmente ou totalmente depletado, dependendo da existência ou não de uma região neutra no canal do transistor e do potencial aplicado ao substrato $\left(\mathrm{V}_{\mathrm{GB}}\right)$ do dispositivo.

\subsection{Modo de Operação}

Quanto à forma de funcionamento, os dispositivos SOI MOSFETs apresentam dois possíveis modos de operação:

- Modo Acumulação

- Modo Enriquecimento ou Inversão

O modo de operação dos transistores depende dos parâmetros de fabricação, onde o mais importante é o material de porta do transistor. A função trabalho do material de porta $\left(\phi_{M}\right)$ influência a tensão de limiar, uma vez que a diferença de função trabalho entre o material de porta e o filme de silício ( $\left.\phi_{M S}\right)$ é igual a $\phi_{M}-\phi_{S i}$, onde $\phi_{S i}$ é a função trabalho do canal de silício. A Tabela 1 apresenta os diferentes modos de operação para os transistores em função do material de porta. 
Tabela 1: Modos de operação SOI MOSFET dependendo do material de porta.

\begin{tabular}{|c|c|c|}
\hline & Porta N+ Poly & Porta P+ Poly \\
\hline Transistor canal N & Inversão & Acumulação \\
\hline Transistor canal P & Acumulação & Inversão \\
\hline
\end{tabular}

Nesse trabalho os dispositivos estudados e utilizados serão do tipo SOI MOSFETs modo inversão, portanto não serão abordados o funcionamento e equacionamento básico dos dispositivos SOI MOSFETs modo acumulação.

\subsection{Material Isolante de Porta}

A integridade do óxido de porta crescido sobre lâminas SOI é similar a do óxido crescido em lâminas convencionais ${ }^{28}$. Assim como as dimensões dos dispositivos são reduzidas, as dimensões do óxido de porta estão se aproximando de espessuras de $1,5 \mathrm{~nm}$ ou menores ${ }^{29}$ para aumentar a corrente do dispositivo, através de um aumento de capacitância do óxido de porta $\left(\mathrm{C}_{\mathrm{oxf}}\right)$. Um efeito desta redução é a ocorrência de tunelamento de elétrons através destes óxidos, desta forma elevando o valor da corrente de porta, não devendo mais ser desprezada ${ }^{30}$. Pode-se aumentar o valor da capacitância do óxido de porta $\left(\mathrm{C}_{\mathrm{oxf}}\right)$ e evitar a influência da corrente de porta através do uso de materiais dielétricos que possuem a constante dielétrica, $\varepsilon$, maior que do óxido de silício $\left(\varepsilon_{\mathrm{SiO} 2}=3,9\right)$. Materiais dielétricos de porta como óxido de hafnium $\left(\mathrm{HfO}_{2}\right)$ e óxido de tântalo $\left(\mathrm{Ta}_{2} \mathrm{O}_{5}\right)$ têm sido usados em dispositivos SOI MOSFETs ${ }^{26}$. Porém ainda há uma desvantagem devido à não consolidação da tecnologia de materiais dielétricos com alta constante dielétrica, que é a baixa qualidade da interface silício-dielétrico, o que causa a degradação da mobilidade $\left(\mu_{n}\right)^{26}$. 


\subsection{Parâmetros Elétricos Básicos}

\subsubsection{Tensão de Limiar}

A tensão de limiar de um transistor MOS convencional modo inversão é classicamente dada pela equação $(4)^{15}$ :

$$
\mathrm{V}_{\mathrm{th}}=\phi_{\mathrm{MS}}-\frac{\mathrm{Q}_{\mathrm{ox}}}{\mathrm{C}_{\mathrm{ox}}}+2 \phi_{\mathrm{Ff}}+\frac{\mathrm{q} \mathrm{N}_{\mathrm{a}} \mathrm{x}_{\mathrm{dmáx}}}{\mathrm{C}_{\mathrm{ox}}}
$$

onde $Q_{\text {ox }}$ é a densidade de carga fixa na interface $\mathrm{Si}_{-} \mathrm{SiO}_{2}$, q é a carga elementar do elétron, $C_{o x}$ é a capacitância do óxido de porta e $\mathrm{N}_{a}$ é a concentração de dopantes da camada de Si.

Para um transistor SOI canal $\mathrm{N}$ de camada espessa, onde $t_{\mathrm{Si}}>$

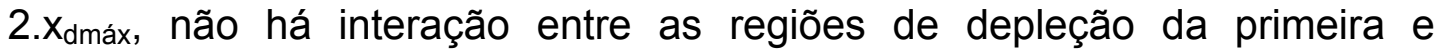
segunda interfaces, a tensão de limiar é obtida pela mesma equação do transistor nMOS convencional ${ }^{15}$. Em dispositivos SOI nMOSFETs modo inversão e totalmente depletados (FD SOI), existe interação entre a primeira e segunda interfaces, a tensão aplicada na porta $\left(V_{G F}\right)$ influência as condições de polarização da segunda interface, do mesmo modo que a tensão aplicada no substrato $\left(\mathrm{V}_{\mathrm{GB}}\right)$ afeta as condições de polarização da primeira interface. Através do modelo clássico de Lim \& Fossum ${ }^{23}$ pode-se descrever por um sistema de equações, (5) e (6), a relação entre as tensões $V_{G F}$ e $V_{G B}$, com os potenciais na primeira $\left(\phi_{S F}\right)$ e segunda $\left(\phi_{S B}\right)$ interfaces.

$$
\mathrm{V}_{\mathrm{GF}}=\phi_{\mathrm{MS} 1}-\frac{\mathrm{Q}_{\text {oxf }}}{\mathrm{C}_{\text {oxf }}}+\left(1+\frac{\mathrm{C}_{\mathrm{Si}}}{\mathrm{C}_{\mathrm{oxf}}}\right) \phi_{\mathrm{SF}}-\frac{\mathrm{C}_{\mathrm{Si}}}{\mathrm{C}_{\text {oxf }}} \phi_{\mathrm{SB}}-\frac{0.5 \mathrm{Q}_{\text {depl }}+\mathrm{Q}_{\text {inv1 }}}{\mathrm{C}_{\text {oxf }}}
$$




$$
\mathrm{V}_{\mathrm{GB}}=\phi_{\mathrm{MS} 2}-\frac{\mathrm{Q}_{\mathrm{oxb}}}{\mathrm{C}_{\mathrm{oxb}}}+\left(1+\frac{\mathrm{C}_{\mathrm{Si}}}{\mathrm{C}_{\mathrm{oxb}}}\right) \phi_{\mathrm{SB}}-\frac{\mathrm{C}_{\mathrm{Si}}}{\mathrm{C}_{\mathrm{oxb}}} \phi_{\mathrm{SF}}-\frac{0.5 \mathrm{Q}_{\mathrm{depl}}+\mathrm{Q}_{\mathrm{S} 2}}{\mathrm{C}_{\mathrm{oxb}}}
$$

Onde $\phi_{\mathrm{MS} 1}$ e $\phi_{\mathrm{MS} 2}$ são as diferenças de função trabalho entre a porta e a camada de silício e entre o substrato e camada de silício, respectivamente, $Q_{\text {inv1 }}$ é a carga de inversão na primeira interface, $Q_{S 2}$ é a carga na superfície da segunda interface, $\phi_{\mathrm{SF}}$ e $\phi_{\mathrm{SB}}$ são os potenciais de superfície da primeira e segunda interfaces, $Q_{\text {oxf }}$ e $Q_{\text {oxb }}$ são as densidades de cargas fixas na primeira e segunda interfaces, $\mathrm{C}_{\text {oxf }}$ e $\mathrm{C}_{\text {oxb }}$ são as capacitâncias dos óxidos de porta e enterrado e $\mathrm{C}_{\mathrm{Si}}$ é a capacitância da camada de $\mathrm{Si}$.

É possível obter três equações diferentes para a tensão de limiar a partir das equações (5) e (6) devido ao fato da segunda interface $\left(V_{G B}\right)$ poder assumir os estados de acumulação, depleção ou inversão. O potencial $\phi_{S B}$ pode variar de 0 a $2 \phi_{F}$ dependendo do valor de $V_{G B}$ quando a segunda interface está depletada. $\quad V_{G B}$,acc , que é o valor de $V_{G B}$ para o qual a segunda interface está acumulada, é obtido pela equação (6) nas condições de $\phi_{\mathrm{SF}}=2 \phi_{\mathrm{F}}, \phi_{\mathrm{SB}} \approx 0$ e $\mathrm{Q}_{\mathrm{S} 2}=0$, portanto a tensão de limiar da primeira interface com a segunda interface na condição de acumulação $\left(\mathrm{V}_{\text {thF,acc2 }}\right)$, será:

$$
\mathrm{V}_{\mathrm{th}_{\mathrm{F}, \mathrm{acc} 2}}=\phi_{\mathrm{MS} 1}-\frac{\mathrm{Q}_{\mathrm{oxf}}}{\mathrm{C}_{\mathrm{oxf}}}+\left(1+\frac{\mathrm{C}_{\mathrm{Si}}}{\mathrm{C}_{\mathrm{oxf}}}\right) 2 \phi_{\mathrm{F}}-\frac{\mathrm{Q}_{\mathrm{depl}}}{2 \mathrm{C}_{\mathrm{oxf}}}
$$

Do mesmo modo, o valor de $\mathrm{V}_{\mathrm{GB}}$ para o qual a segunda interface está invertida, $V_{\mathrm{GB}}$,inv ,é dado pela mesma equação (6) nas condições de $\phi_{\mathrm{SF}}=$ $2 \phi_{\mathrm{F}}, \phi_{\mathrm{SB}}=2 \phi_{\mathrm{F}}$ e $\mathrm{Q}_{\mathrm{S} 2}=0$, e assim obtendo-se a tensão de limiar da primeira

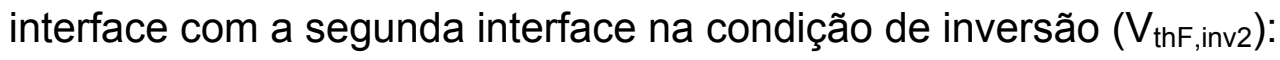




$$
\mathrm{V}_{\mathrm{th}_{\mathrm{F}, \text { inv2 }}}=\phi_{\mathrm{MS} 1}-\frac{\mathrm{Q}_{\mathrm{oxf}}}{\mathrm{C}_{\mathrm{oxf}}}+2 \phi_{\mathrm{F}}-\frac{\mathrm{Q}_{\mathrm{dep} 1}}{2 \mathrm{C}_{\mathrm{oxf}}}
$$

E através da combinação das equações (5) e (6) na situação de $\mathrm{V}_{\mathrm{GB}, \text { acc }}<\mathrm{V}_{\mathrm{GB}}<\mathrm{V}_{\mathrm{GB} \text {,inv }}$, obtém-se a expressão da tensão de limiar da primeira interface com a segunda interface depletada $\left(\mathrm{V}_{\mathrm{thF}}\right.$, dep12), que pode ser expressa por (9).

$$
\mathrm{V}_{\text {th }_{\mathrm{F}, \mathrm{dep} 12}}=\mathrm{V}_{\text {th }_{\mathrm{F}, \text { acc2 }}}-\frac{\mathrm{C}_{\mathrm{Si}} \mathrm{C}_{\mathrm{oxb}}}{\mathrm{C}_{\mathrm{oxf}}\left(\mathrm{C}_{\mathrm{Si}}+\mathrm{C}_{\mathrm{oxb}}\right)}\left(\mathrm{V}_{\mathrm{GB}}-\mathrm{V}_{\mathrm{GB}, \mathrm{acc}}\right)
$$

\subsubsection{Corrente de Dreno e Inclinação de Sublimiar}

Grande parte das vantagens oferecidas pelo transistor SOI totalmente depletado (FD-SOI) sobre os transistores SOI parcialmente depletados (PDSOI) e os transistores MOS convencionais está relacionada ao efeito de corpo ("body factor"), o qual afeta diretamente a capacidade de fornecimento de corrente do transistor e sua inclinação de sublimiar ${ }^{31,32}$.

O efeito de corpo em um dispositivo convencional MOS ("bulk") é definido como a dependência da tensão de limiar com as condições de polarização do substrato. Em um transistor SOI, o efeito pode ser similarmente definido como a dependência de tensão de limiar $\left(\mathrm{V}_{\mathrm{thF}}\right)$ com as condições de polarização do substrato $\left(\mathrm{V}_{\mathrm{GB}}\right)$. Sob o ponto de vista das interações que ocorrem no filme de silício, em uma aproximação inicial, o efeito de corpo pode ser compreendido como a efetividade de controle através da porta no potencial de superfície da interface óxido de porta/silício. 
A relação do acoplamento capacitivo do transistor MOS indica de que forma atua o efeito de corpo (n), demonstrada através da equação (10):

$$
n=1+\alpha
$$

onde

$\alpha=\frac{C_{D}}{C_{o x}}$ para transistores SOI parcialmente depletados e MOS convencionais.

$\alpha=\frac{\mathrm{C}_{\mathrm{Si}}}{\mathrm{C}_{\mathrm{oxf}}}$ quando a segunda interface está em acumulação;

$\alpha=\frac{\mathrm{C}_{\mathrm{Si}} \mathrm{C}_{\mathrm{oxb}}}{\mathrm{C}_{\mathrm{oxf}}\left(\mathrm{C}_{\mathrm{Si}}+\mathrm{C}_{\mathrm{oxb}}\right)}$ quando a segunda interface está em depleção;

onde $\mathrm{C}_{\mathrm{oxf}}\left(\mathrm{C}_{\mathrm{ox}}\right.$ para os transistores MOS convencionais), é a capacitância do óxido de porta, $\mathrm{C}_{\mathrm{oxb}}$ é a capacitância do óxido enterrado, $\mathrm{C}_{\mathrm{Si}}$ é a capacitância da camada de silício e $C_{D}$ é a capacitância da região de depleção, todas por unidade de área, e são expressos por:

$$
\mathrm{C}_{\mathrm{oxf}}=\frac{\varepsilon_{\mathrm{ox}}}{\mathrm{t}_{\mathrm{oxf}}} ; \quad \mathrm{C}_{\mathrm{oxb}}=\frac{\varepsilon_{\mathrm{ox}}}{\mathrm{t}_{\mathrm{oxb}}} ; \quad \mathrm{C}_{\mathrm{Si}}=\frac{\varepsilon_{\mathrm{Si}}}{\mathrm{t}_{\mathrm{Si}}} \text { e } \mathrm{C}_{\mathrm{D}}=\frac{\varepsilon_{\mathrm{Si}}}{\mathrm{x}_{\mathrm{dmáx}}}
$$

Através da equação (10) determina-se o valor de $n$ para transistores SOI totalmente depletados que é tipicamente 1,05, diferente da faixa de variação entre 1,15 e 1,7 para os transistores MOS convencionais ${ }^{33}$. O menor efeito de corpo causa implicações nas características de saída dos dispositivos, o que pode ser observado a partir da equação simplificada para a corrente de dreno $\left(I_{\mathrm{DS}}\right)$ dos transistores, em cada uma das regiões de operação $^{32}$ : 
Região de Triodo:

$$
\mathrm{I}_{\mathrm{DS}} \cong \frac{\mathrm{W} \mu_{\mathrm{n}} \mathrm{C}_{\mathrm{oxf}}}{\mathrm{L}}\left[\left(\mathrm{V}_{\mathrm{GF}}-\mathrm{V}_{\mathrm{thF}}\right) \mathrm{V}_{\mathrm{DS}}-\mathrm{n} \frac{\mathrm{V}_{\mathrm{DS}}^{2}}{2}\right]
$$

Região de Saturação:

$$
\mathrm{I}_{\mathrm{DS}} \cong \frac{\mathrm{W} \mu_{\mathrm{n}} \mathrm{C}_{\mathrm{oxf}}}{2 \mathrm{Ln}}\left(\mathrm{V}_{\mathrm{GF}}-\mathrm{V}_{\mathrm{thF}}\right)^{2}
$$

onde W e L são a largura e comprimento de canal do transistor, $\mu_{\mathrm{n}}$ é a mobilidade efetiva dos elétrons na região de canal e $V_{\text {thF }}$ é a tensão de limiar do transistor SOI.

O fato de ocorrer um menor efeito de corpo também está relacionado a uma outra influência importante, o fator de inclinação de sublimiar $S^{23}$ dos dispositivos, definido como o inverso da inclinação da curva log $\left(\mathrm{I}_{\mathrm{DS}}\right)$ em função da tensão aplicada a porta $\left(\mathrm{V}_{\mathrm{GF}}\right)$ no regime de sublimiar, Figura 5.

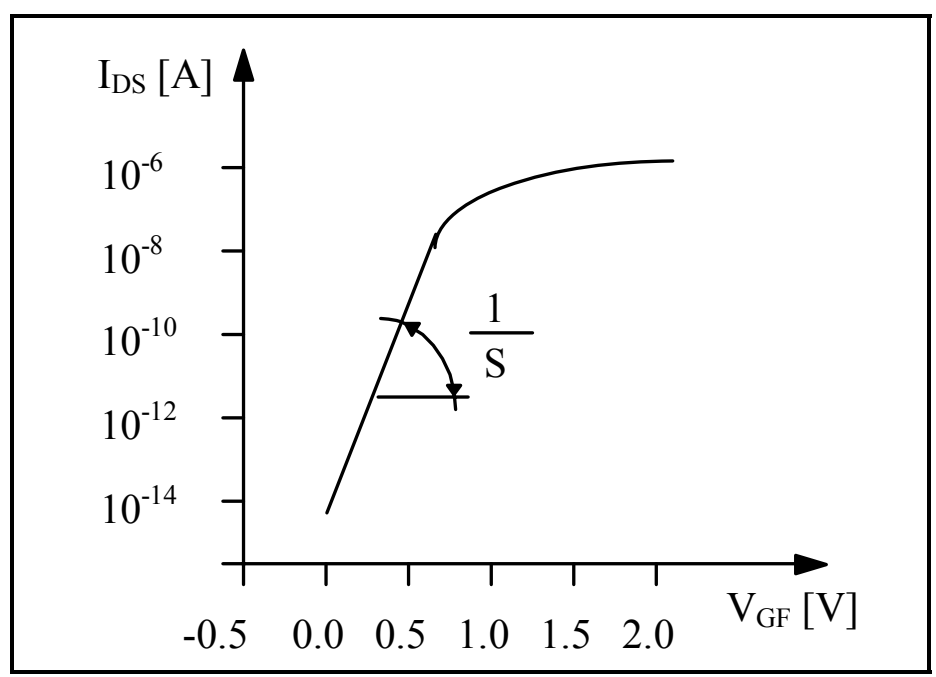

Figura 5 - Obtenção do fator de inclinação de sublimiar S em um transistor nMOS. 
O valor de S pode ser expresso, sob aproximações, considerando desprezível a influência das armadilhas de interface, pela equação $(13)^{34,35}$ :

$$
\mathrm{S}=\frac{\mathrm{kT}}{\mathrm{q}} \ln (10) \mathrm{n}
$$

Valores típicos de $\mathrm{S}$ encontrados em transistores MOS convencionais e SOI MOS totalmente depletados são mostrados na Figura 6. O excelente valor do fator de inclinação de sublimiar em um dispositivo SOI MOS de camada fina e totalmente depletado permite usar valores menores da tensão de limiar do que o dispositivo MOS convencional sem aumento de corrente de fuga, resultando numa melhora do desempenho dos dispositivos em alta velocidade.

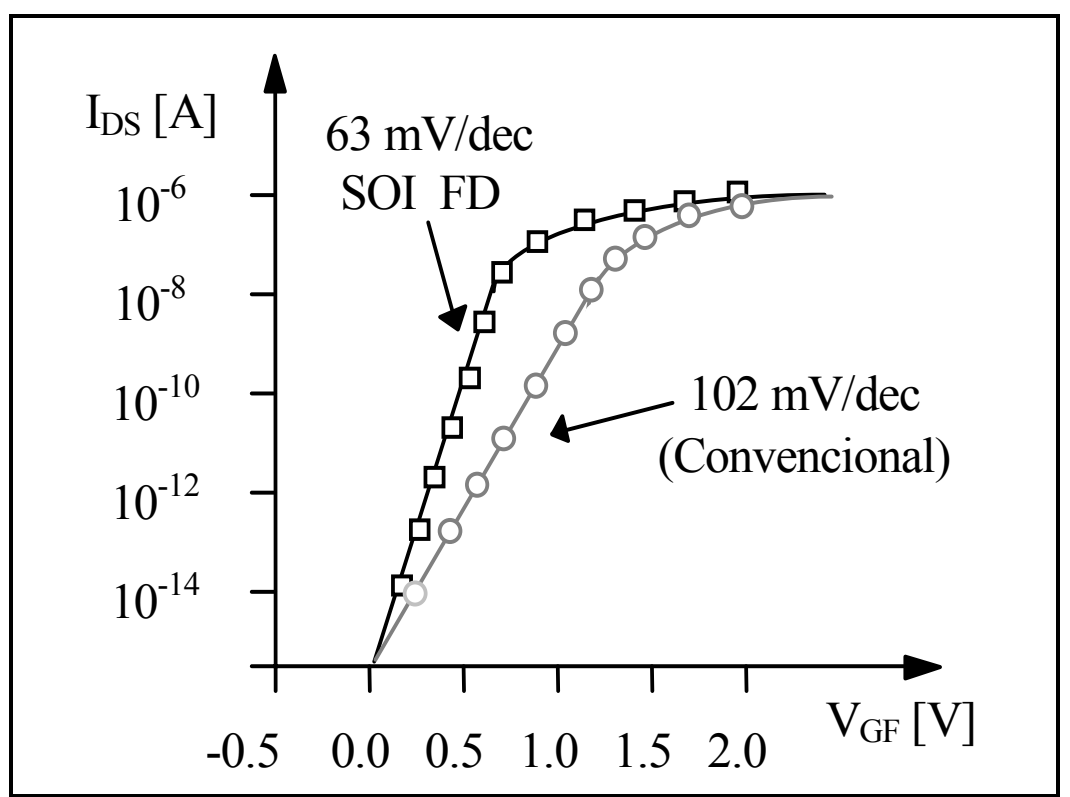

Figura 6 - Valores típicos de S encontrados em transistores MOS convencionais e SOI MOS totalmente depletados (FD) $)^{23}$ 


\subsubsection{Mobilidade dos portadores}

A mobilidade dos elétrons $\left(\mu_{n}\right)$ no canal de um transistor é um dos parâmetros fundamentais na tecnologia MOS, junto à tensão de limiar $\left(\mathrm{V}_{\mathrm{th}}\right)$. Além da mobilidade dos elétrons determinar o nível de corrente em um dispositivo e sua transcondutância, é responsável pelo tempo de resposta em circuitos lógicos ${ }^{36}$.

A mobilidade efetiva dos elétrons na camada de inversão de um transistor MOS de canal $\mathrm{N}$ é influênciada pelo campo elétrico vertical que atua abaixo do óxido de porta ${ }^{23}$. A dependência da mobilidade com a tensão aplicada ao terminal de porta $\mathrm{V}_{\mathrm{GS}}$ pode ser dada pelo modelo convencional de mobilidade ${ }^{14}$ :

$$
\mu_{\mathrm{n}}=\frac{\mu_{0}}{1+\theta\left(\mathrm{V}_{\mathrm{GS}}-\mathrm{V}_{\mathrm{th}}\right)}
$$

onde $\mu_{\mathrm{n}}$ é a mobilidade em função de $\mathrm{V}_{\mathrm{GS}}, \mathrm{V}_{\text {th }}$ é a tensão de limiar, $\theta$ é o coeficiente de degradação de mobilidade pelo campo elétrico vertical ${ }^{14}, \mu_{0}$ é a mobilidade para baixo campo elétrico (note que $\mu_{0}=\mu_{n}$ para $V_{G S}=V_{\text {th }}$ ), e através de uma expressão empírica ${ }^{14}$ pode-se descrever $\theta$ como:

$$
\theta=\frac{\beta_{0}}{t_{o x}}
$$

onde o parâmetro $\beta_{0}$ varia tipicamente entre 0.001 and $0.004 \mathrm{~mm} / \mathrm{V}^{37}$, e $\mathrm{t}_{\mathrm{ox}}$ é a espessura do óxido de porta. Desta forma, a degradação da mobilidade pode ser considerada na equação da corrente de dreno de um MOSFET para os dois modos de operação: 


$$
\mathrm{I}_{\mathrm{DS}}=\frac{\mu_{0}}{1+\theta\left(\mathrm{V}_{\mathrm{GF}}-\mathrm{V}_{\mathrm{thF}}\right)} \frac{\mathrm{W}}{\mathrm{L}} \mathrm{C}_{\mathrm{oxf}}\left[\left(\mathrm{V}_{\mathrm{GF}}-\mathrm{V}_{\mathrm{thF}}\right) \mathrm{V}_{\mathrm{DS}}-\mathrm{n} \frac{\mathrm{V}_{\mathrm{DS}}^{2}}{2}\right]
$$

em regime de triodo;

$$
\mathrm{I}_{\mathrm{DS}}=\frac{1}{2 \mathrm{n}} \frac{\mu_{0}}{1+\theta\left(\mathrm{V}_{\mathrm{GF}}-\mathrm{V}_{\mathrm{thF}}\right)} \frac{\mathrm{W}}{\mathrm{L}} \mathrm{C}_{\mathrm{oxf}}\left(\mathrm{V}_{\mathrm{GF}}-\mathrm{V}_{\mathrm{thF}}\right)^{2}
$$

em regime de saturação.

A dependência da mobilidade efetiva dos elétrons com a temperatura é dada pela relação ${ }^{14,15}$ :

$$
\mu_{\mathrm{n}}(\mathrm{T})=\mu_{\mathrm{n}}\left(\mathrm{T}_{0}\right)\left(\frac{\mathrm{T}_{0}}{\mathrm{~T}}\right)^{\mathrm{c}}
$$

onde $T_{0}$ é temperatura ambiente em valor absoluto $(K)$, em que $T>T_{0}$, e $c$ é a constante empírica ${ }^{14}$ que varia de 1,5 a 2,4.

\subsection{Métodos de Extração de Parâmetros}

Por melhor que seja um modelo, ele não fornecerá resultados precisos se os valores usados para seus parâmetros não forem adequados. Os parâmetros devem ser tais que os resultados concordem com dados experimentais dentro de uma faixa de erro determinada. Para tanto, é necessário estabelecer um procedimento para, a partir de determinadas medidas sobre os dispositivos, obter valores adequados dos parâmetros do modelo. 
Neste tópico serão apresentados os métodos de extração dos parâmetros de maior influência na determinação da tensão de polarização no ponto invariante com a temperatura $\left(\mathrm{V}_{\mathrm{ZTC}}\right)$.

\subsubsection{Tensão de Limiar $\left(\mathrm{V}_{\mathrm{thF}}\right)$}

A tensão de limiar $V_{\text {th }}$, que é um importante parâmetro para a modelagem, simulação e caracterização de dispositivos MOSFET, é convencionalmente definida como a tensão na porta $\left(\mathrm{V}_{\mathrm{GF}}\right)$, que causa o inicio da inversão forte no canal dos MOSFETs. O valor da tensão de limiar pode ser extraído de medidas experimentais tanto de características da corrente de dreno ou da capacitância, usando um ou mais transistores.

$\mathrm{O}$ método usado para extração de $\mathrm{V}_{\text {thF }}$ com o transistor operando na região linear foi o da Segunda Derivada $(S D)^{38}$. O método $S D$, foi desenvolvido para evitar a dependência com a resistência série, e determina a tensão de limiar $\left(\mathrm{V}_{\mathrm{thF}}\right)$ como a tensão na porta $\left(\mathrm{V}_{\mathrm{GF}}\right)$ no ponto em que a derivada da transcondutância é máxima (ou seja, $d g_{m} / d V_{G F}=d^{2} I_{D S} / d V_{G F}{ }^{2}$ ). A Figura 7 mostra a implementação deste método para um dispositivo PD SOI nMOSFET, extraindo o valor de $\mathrm{V}_{\text {thF }}$ pela indicação do valor máximo de $\mathrm{dg}_{\mathrm{m}} / \mathrm{dV} \mathrm{GF}$. 


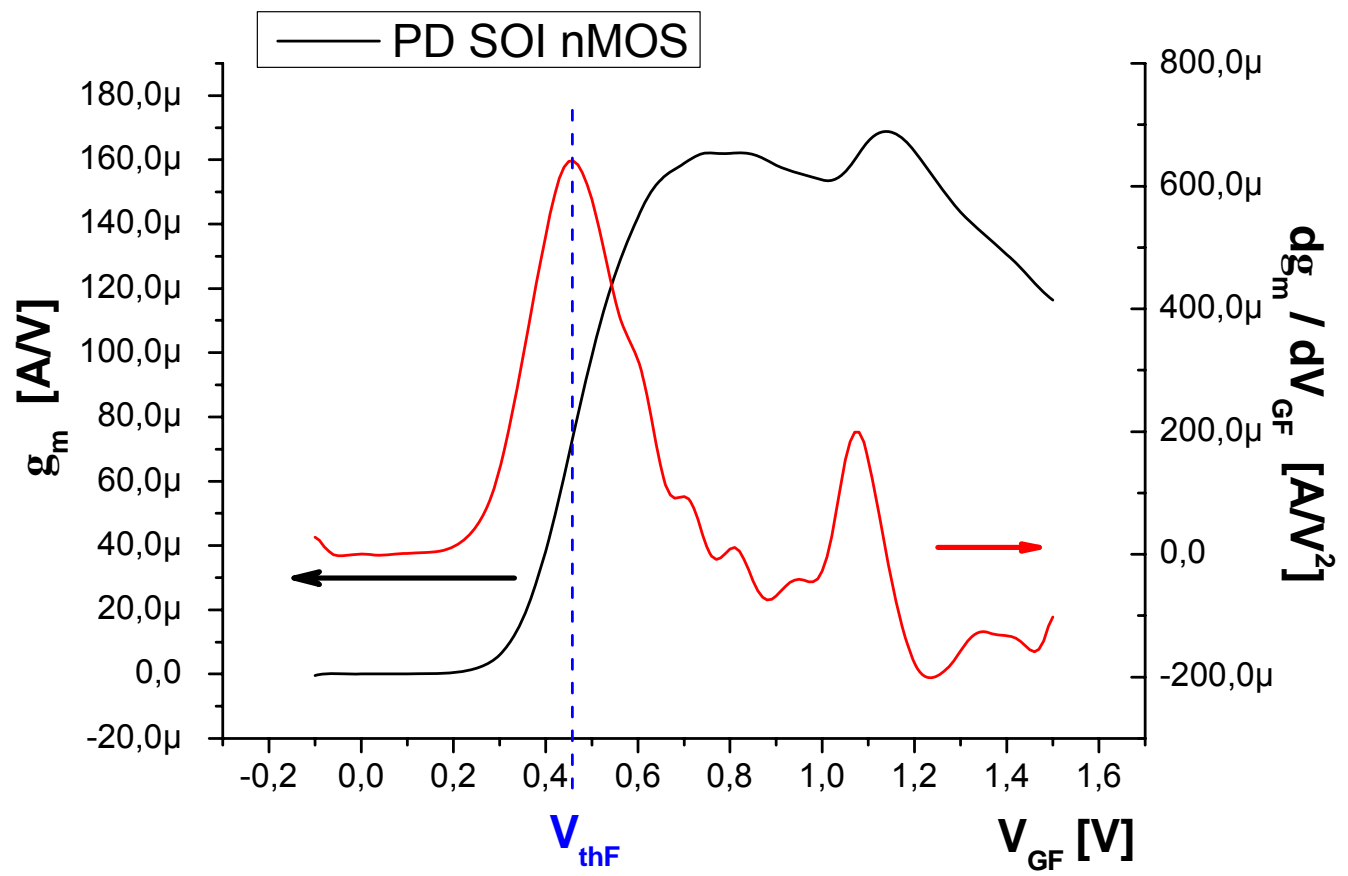

Figura 7 - Extração de $\mathrm{V}_{\text {thF }}$ usando o método da segunda derivada (SD) para um dispositivo operando na região linear.

O segundo método ${ }^{14}$ usado para obtenção do valor da tensão de limiar $\left(\mathrm{V}_{\text {thF }}\right)$ também é através do uso da extrapolação linear. A corrente de dreno é medida em diferentes valores de $\mathrm{V}_{\mathrm{GF}}$, em tensões de dreno $\left(\mathrm{V}_{\mathrm{DS}}\right)$ que garantam a operação do transistor na região de saturação $\left(V_{D S}>=V_{G F}-\right.$ $\left.\mathrm{V}_{\mathrm{thF}}\right)$.

A corrente de dreno em modo saturação obedece a lei quadrática, e quando não considerado o efeito de canal curto, pode-se aproximar para:

$$
\mathrm{I}_{\mathrm{DS}}=\frac{\mathrm{W}}{\mathrm{L}} \mathrm{C}_{\mathrm{oxf}} \mu_{\mathrm{n}} \frac{1}{2}\left(\mathrm{~V}_{\mathrm{GF}}-\mathrm{V}_{\mathrm{thF}}\right)^{2}
$$


Obtendo-se o gráfico da raiz quadrada da corrente de dreno (IDS) versus a tensão de porta $\left(\mathrm{V}_{\mathrm{GF}}\right)$ resulta-se em uma curva com variação linear, e através da extrapolação da reta determina-se o ponto que intercepta o eixo das coordenadas $\mathrm{V}_{\mathrm{GF}}$ resultando no ponto do valor de $\mathrm{V}_{\text {thF }}$, como mostrado na Figura 8.

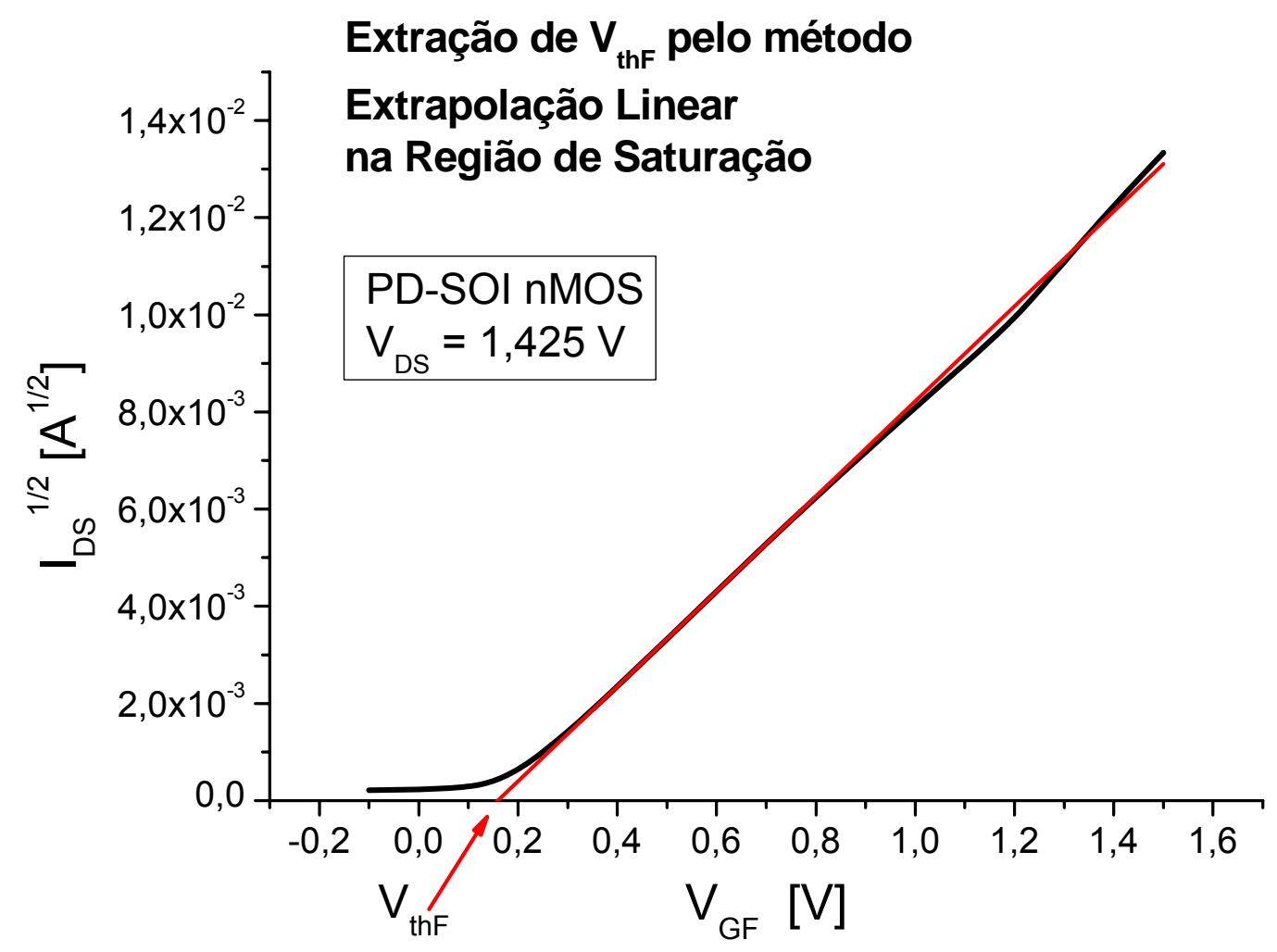

Figura 8 - Extração de $\bigvee_{\text {thF }}$ usando o método de extrapolação linear para um dispositivo de canal curto operando na região de saturação. 


\subsubsection{Mobilidade Efetiva no Canal do Transistor}

Para a determinação da mobilidade dos portadores para baixo campo $\left(\mu_{0}\right)$ de um transistor MOSFET, obtém-se a transcondutância $\left(\mathrm{g}_{\mathrm{m}}\right)$ através da equação (20).

Derivando-se a equação (11)

$$
\mathrm{g}_{\mathrm{m}}=\left.\frac{\partial \mathrm{I}_{\mathrm{DS}}}{\partial \mathrm{V}_{\mathrm{GF}}}\right|_{\mathrm{V}_{\mathrm{DS}}=\text { baixo }}
$$

Isolando-se $\mu_{\mathrm{n}}$ fica:

$$
\mu_{\mathrm{n}}=\frac{\mathrm{L}}{\mathrm{WC}_{\mathrm{oxf}} \mathrm{V}_{\mathrm{DS}}} \mathrm{g}_{\mathrm{m}}
$$

A partir da equação (21), estabelece-se uma relação linear entre a mobilidade e a transcondutância. Como $V_{D S}$ é pequeno e, na região linear da curva, $V_{G S}$ também é pequeno, o valor da mobilidade encontrada será independente tanto do campo horizontal quanto do vertical, respectivamente, atingindo seu valor máximo. Essa mobilidade é obtida no ponto de máximo da transcondutância $g_{m}$ max.

Na Figura 9, observa-se que após atingir-se o valor máximo da transcondutância e, por conseqüência, da mobilidade $\left(\mu_{0}\right)$, a transcondutância começa a sofrer uma degradação. 


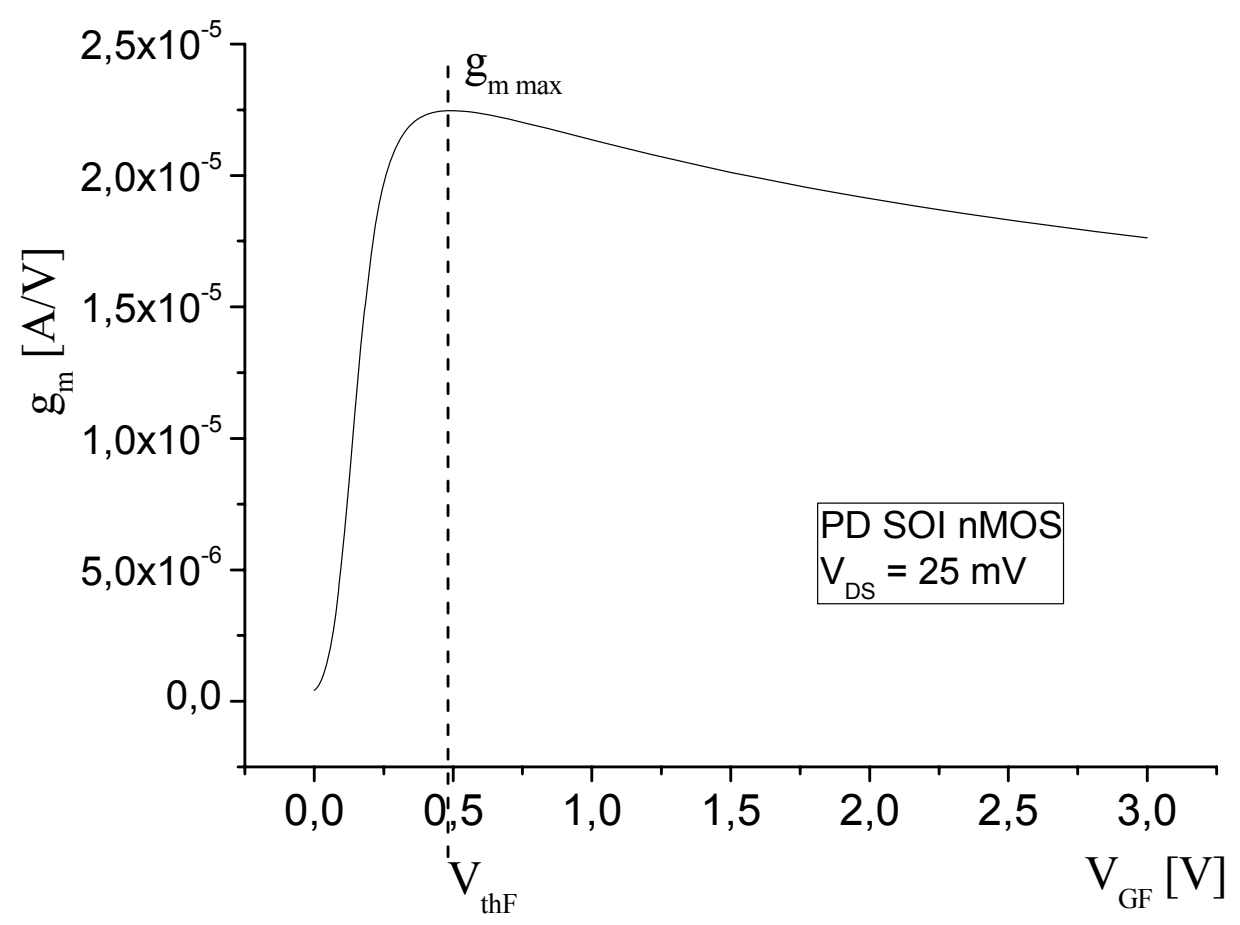

Figura 9 - Extração de $V_{\text {thF }}$ usando o método de máxima transcondutância $\left(G_{M \max }\right)$ para um dispositivo operando na região linear.

A determinação da mobilidade de saturação $\left(\underline{\mu}_{\text {sat }}\right)^{39}$, ou seja, quando o transistor MOSFET está operando na região de saturação, foi obtida através da mesma metodologia utilizada para a extração da tensão de limiar, em que equação da corrente de dreno em modo saturação, equação (19), obedece a lei quadrática. Desta forma, com os dados obtidos experimentalmente, é traçado o gráfico da raiz quadrada da corrente de dreno (IDS) versus a tensão na porta $\left(V_{\mathrm{GF}}\right)$, e desta forma extrai-se a inclinação $(\mathrm{m})$ da reta obtida, portanto $\mu_{\text {sat: }}$ :

$$
\mu_{\mathrm{sat}}=\frac{2 \mathrm{~L} \mathrm{~m}^{2}}{\mathrm{WC}_{\mathrm{oxf}}}
$$




\subsection{Aplicações em Altas Temperaturas e a Tecnologia SOI}

Durante as últimas décadas, o interesse no desenvolvimento de circuitos e dispositivos eletrônicos em alta temperatura tem atraído muitas pesquisas das indústrias, universidades e do governo em todo mundo. Embora as motivações individuais, necessidades e aplicações tenham sido relativamente diferentes, os requisitos comuns dos dispositivos e circuitos que emergiram dos seus esforços, atualmente constituem as regras informais de um sucesso da tecnologia em alta temperatura. Um esboço, quantitativo destes requisitos, é que a maioria das aplicações, circuitos e dispositivos encapsulados devem ser capazes de operar confiavelmente em campo por um mínimo de 1000 horas à temperaturas até $300^{\circ} \mathrm{C}$ nas junções físicas e resistir choques, vibrações e uma larga faixa de pressão atmosférica.

A indústria automobilística, que é um exemplo de área e mercado com grande potencial e aplicações que atuam em altas temperaturas, possui primariamente a intenção de melhorar o controle e monitoramento do motor. Desta maneira colaborando para otimizar a operação do motor, reduzir emissão de poluentes e proporcionar melhor economia de combustível. A chamada eletrônica embarcada, neste setor para ser considerada confiável, deve ser capaz de estender sua operação em todas as estações do ano (ou quase) em qualquer lugar do globo terrestre, tanto quanto resistir a muitos ciclos de variação de temperatura (noite-dia / deserto quente / montanha frio). Além do mais, com a tendência da indústria sempre buscar uma melhor forma aerodinâmica dos veículos, são esperadas temperaturas maiores embarcadas, da qual a faixa atual típica extrema está entre $-40^{\circ} \mathrm{C}$ e $200^{\circ} \mathrm{C}$, dependendo do local especifico do bloco do motor ou do sistema de exaustão ${ }^{40,41,42,43}$. 
As indústrias de perfuração e exploração geotérmica necessitam de circuitos que trabalham em altas temperaturas nas ferramentas de perfuração ou sondas usadas para localizar e avaliar compostos em reservatórios profundos dentro da superfície do globo terrestre (mais de 10.000 pés de profundidade). Uma vez que o volume disponível dentro destas ferramentas é escasso onde ficam os circuitos, e devido ao típico trabalho de perfuração exigir operações em ambientes com temperaturas até $300^{\circ} \mathrm{C}$ por dezenas de horas em cada tentativa, estas industrias têm colocado uma importante ênfase na identificação e aplicação de tecnologias de circuitos integrados para altas temperaturas em suas necessidades ${ }^{44}$.

A indústria de motores para aviação civil e militar e a indústria de exploração espacial necessitam de circuitos eletrônicos de alta temperatura, para finalidades de monitoramento da performance de motores, tanto para os estágios de desenvolvimento e operacional na exploração remota de atmosferas de planetas e sondas solares. As exigências típicas em tais aplicações incluem que sejam equipamentos leves, circuitos de baixa potência e que possam resistir por um longo tempo em operação até $600^{\circ} \mathrm{C}^{44}$.

As indústrias de reatores nucleares e elétricos estão interessadas em aplicações de monitoramento, controle e segurança para usinas de energia. $O$ interesse de circuitos que operam em altas temperaturas e ambientes com radiação é motivado pela necessidade de utilizar instrumentos de detecção confiáveis em locais da usina que são hostis e perigosos para operadores humanos.

A tecnologia de Silício sobre Isolante (Silicon-on-Insulator - SOI) surgiu como a solução mais madura para aplicações em altas temperaturas nas aplicações com tecnologia MOS. Alguns dos fatores que promovem a tecnologia SOI a essa condição são o fato de apresentar um valor menor da corrente de fuga em altas temperaturas do que os dispositivos da tecnologia convencional MOS ("bulk"), assim como também apresenta uma menor 
variação da tensão de limiar com a temperatura ${ }^{45}$. Nesta tecnologia os dispositivos mostram-se imunes ao efeito tiristor parasitário ("latch-up") induzido pela temperatura, e desta forma os circuitos com estes dispositivos podem operar em temperaturas acima de $300^{\circ} \mathrm{C}$, enquanto que na tecnologia convencional ("bulk") é normalmente limitado a $150^{\circ} \mathrm{C}^{26,46}$. 


\section{Ponto Invariante com a Temperatura ZTC ("Zero Temperature Coefficient")}

No presente capítulo será apresentada uma revisão bibliográfica sobre $\mathrm{o}$ ponto invariante com a temperatura (ZTC), a importância da polarização dos dispositivos no ponto ZTC e os modelos existentes na literatura para estudo dos aspectos do comportamento da polarização no ponto invariante com a temperatura $\left(\mathrm{V}_{\mathrm{ZTC}}\right)$ nos regimes de triodo e saturação.

\subsection{Importância da determinação do ponto invariante com a temperatura $\left(\mathrm{V}_{\mathrm{ZTC}}\right)$}

Há um crescimento significativo do número indústrias de circuitos eletrônicos integrados que precisam operar com confiabilidade por um grande período sob um ambiente em condições severas. Alguns desses ambientes são a eletrônica embarcada na indústria automobilística, os equipamentos eletrônicos para exploração espacial e terrestre (pesquisas geotérmicas), a indústria de aeronaves e nuclear ${ }^{16}$.

Nos últimos anos, progressos significativos têm sido realizados nos processos de caracterização do comportamento elétrico de transistores na tecnologia CMOS operando sob uma larga faixa de temperatura ${ }^{16-20}$. E para o caso de circuitos projetados para aplicações em alta temperatura, é desejável que funcionem em um ponto de polarização onde o valor da corrente de dreno $\left(\mathrm{I}_{\mathrm{DS}}\right)$ não apresente variação com a temperatura. Este critério garante que parâmetros do circuito polarizado, tais como por exemplo o desvio da tensão de "offset" em circuitos amplificadores 
operacionais, sejam menos sensíveis no casamento das características dos dispositivos $^{21}$.

A Figura 10 mostra a curva característica, $I_{D S} \times V_{G F}$ (para $V_{D S}=25$ $\mathrm{mV}$ ) de um transistor SOI MOSFET parcialmente depletado (PD-SOI), onde observa-se claramente a existência de um ponto de polarização $V_{\mathrm{GF}}=\mathrm{V}_{\text {ZTC }}$ em que a corrente de dreno $\left(I_{D S}=I_{Z T C}\right)$ é a mesma com a variação de temperatura.

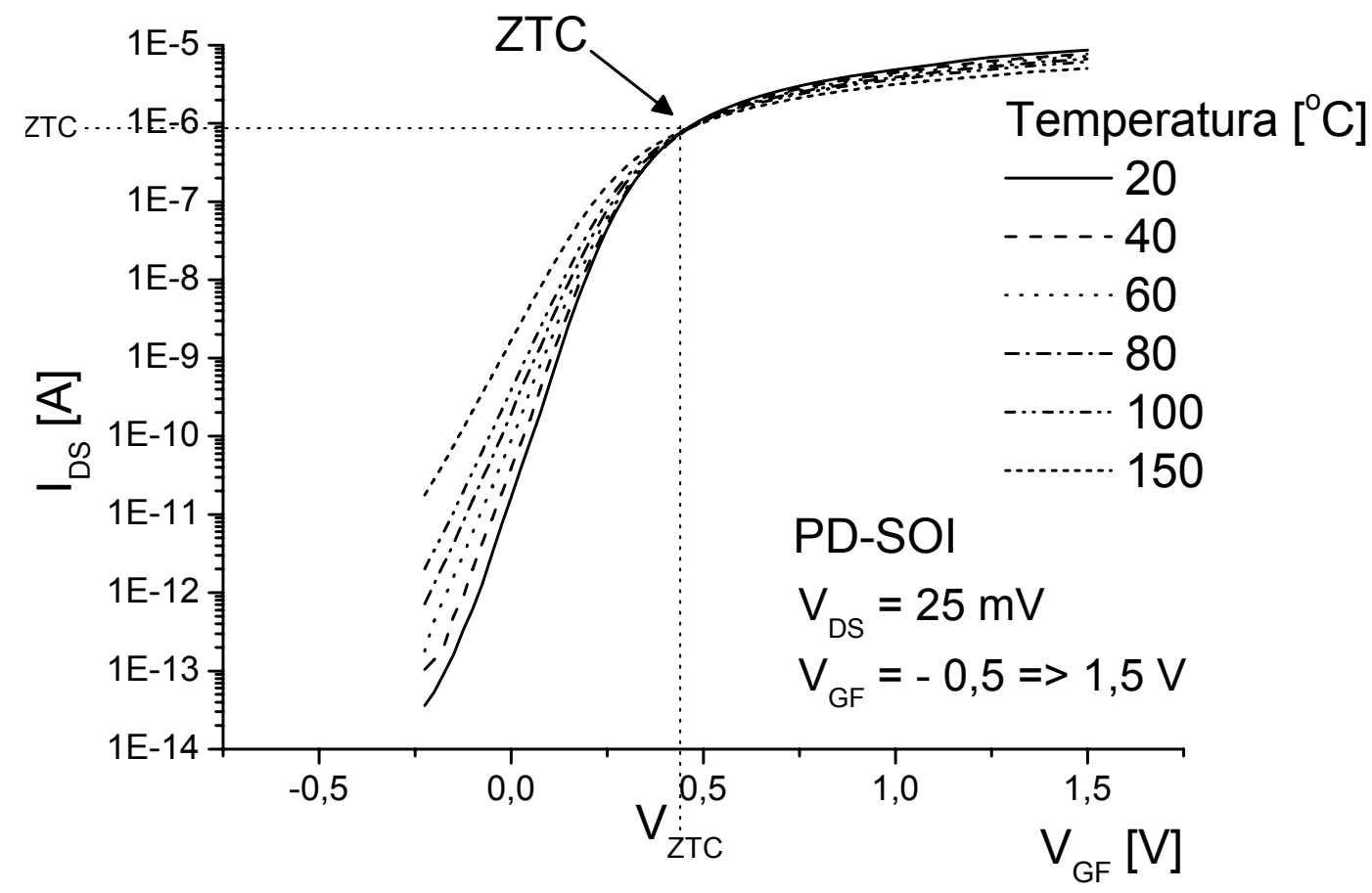

Figura 10: Curva característica $I_{D S} \times V_{G F}$ para um transistor SOI MOSFET parcialmente depletado para diversas temperaturas.

Esse ponto de polarização, que é muito importante para a estabilidade de um circuito em operação sobre uma larga faixa de temperatura, é conhecido como Ponto Invariante com a Temperatura (ZTC - "Zero Temperature Coefficient"). Muitas pesquisas tem abordado o estudo da polarização no ponto ZTC em dispositivos MOS convencionais ${ }^{16,22}$ e dispositivos SOI parcialmente depletados (PD) ${ }^{21}$, considerando a dependência da tensão de limiar $\left(\mathrm{V}_{\mathrm{thF}}\right)$ e da mobilidade $\left(\mu_{\mathrm{n}}\right)$ com a 
temperatura ${ }^{16}$, incluindo o efeito de corpo $(n)^{22}$ e também a degradação da mobilidade com o campo elétrico transversal $(\theta)^{21}$.

A tecnologia SOI CMOS tem sido amplamente usada em aplicações de alta temperatura devido as suas melhores características ${ }^{47,48,49}$ como baixo valor da corrente de fuga, melhor fator de inclinação de sublimiar e supressão do efeito tiristor parasitário ("latch-up") comparado aos dispositivos com tecnologia MOS convencional ${ }^{26,49}$.

\subsection{Definição do Ponto Invariante com a Temperatura (ZTC)}

O funcionamento dos transistores MOS convencionais é fortemente dependente da temperatura ${ }^{14,15}$, e um dos parâmetros responsáveis por esta dependência é a mobilidade efetiva ( $\mu_{\text {eff }}$ ), a qual sabe-se que diminui com aumento da temperatura. Uma aproximação comumente usada é a mostrada na equação (23).

$$
\mu_{\mathrm{eff}}(\mathrm{T})=\mu_{\mathrm{eff}}\left(\mathrm{T}_{0}\right)\left(\frac{\mathrm{T}_{0}}{\mathrm{~T}}\right)^{c}
$$

onde $T_{0}$ é a temperatura ambiente em valor absoluto $(K), T$ é a temperatura de operação em valor absoluto $(K)$ e $c$ é uma constante, que pode assumir valores entre 1.5 e $2.4^{14,15}$.

Outros parâmetros importantes dependentes da temperatura são o potencial de Fermi $\left(\phi_{F}\right)$ e conseqüentemente a tensão de faixa plana $\mathrm{V}_{\mathrm{FB}} \mathrm{A}$ tensão $V_{F B}$ é dependente da temperatura através da diferença de função 
trabalho $\phi_{\mathrm{MS}}$, assumindo a aproximação com a carga $Q_{\mathrm{ox}}$ invariante $^{14}$. Estes efeitos se manifestam na tensão de limiar $V_{\text {th }}$, a qual exibe uma diminuição de maneira aproximadamente linear com o aumento da temperatura, que pode ser aproximada pela equação $(24)^{14}$ :

$$
\mathrm{V}_{\mathrm{th}}(\mathrm{T})=\mathrm{V}_{\mathrm{th}}\left(\mathrm{T}_{0}\right)-d\left(\mathrm{~T}-\mathrm{T}_{0}\right)
$$

onde $d$ está usualmente entre $0.5 \mathrm{mV} / \mathrm{K}$ e $4 \mathrm{mV} / \mathrm{K}$, em que os maiores valores são correspondentes a dispositivos com substratos com alta concentração de impurezas e óxidos de porta espessos.

A existência de um ponto ZTC estável tem sido estudada e comprovada analítica e experimentalmente para transistores MOS convencionais operando nas regiões linear e de saturação de polarização 18,50. A variação sofrida na tensão de limiar e na mobilidade efetiva dos elétrons com a temperatura causará um deslocamento na curva característica do transistor, observada através da variação da corrente de dreno (lDS) em função da variação da tensão aplicada à porta $\left(V_{G F}\right)$, considerando que a degradação da mobilidade dos portadores de carga causará uma diminuição na inclinação desta curva.

Em um certo ponto de polarização da tensão de porta $V_{G F}$, as mudanças nos dois parâmetros supracitados ( $\left.\mathrm{V}_{\text {thF }} \mathrm{e} \mu_{\mathrm{eff}}\right)$ provocam um comportamento compensatório, ou seja, com aumento da temperatura o valor de $V_{\text {th }}$ diminui (aumentando a corrente $I_{D S}$ ) e $\mu_{\text {eff }}$ degrada (diminuindo a corrente $I_{D S}$ ). Desta forma, demonstra-se que existe um determinado ponto de polarização $\left(\mathrm{V}_{\mathrm{ZTC}}\right)$, a corrente $\mathrm{I}_{\mathrm{DS}}\left(\mathrm{V}_{\mathrm{GF}}=\mathrm{V}_{\mathrm{ZTC}}\right)=\mathrm{I}_{\mathrm{ZTC}}$ fica praticamente constante.

Considerando o efeito que ocorre no ponto de polarização ZTC no comportamento de transistores com tecnologia MOS, vários estudos têm 
sido realizados em dispositivos CMOS para desenvolver modelos analíticos para uma polarização ideal da tensão de porta $\left(V_{G S}\right)$ que garanta uma corrente de dreno independente da temperatura em ambas as regiões de operação: linear e saturação ${ }^{16,22,51,52}$.

A determinação do ponto invariante com a temperatura (ZTC) é definida, como o ponto em que o valor da tensão de porta do transistor $\left(V_{G S}\right)$ garante um valor constante para a corrente de dreno $\left(I_{D S}\right)$ com variação da temperatura, portanto; a derivada da corrente com a temperatura neste ponto é nula

$$
\left.\frac{\mathrm{dI}}{\mathrm{dT}}\right|_{\mathrm{V}_{\mathrm{GF}}=\mathrm{V}_{\mathrm{ZTC}}}=0
$$

A relação (25) é a condição necessária para garantir a existência do ponto ZTC.

\subsection{Modelos sobre o Ponto Invariante com a Temperatura (ZTC)}

\subsubsection{Modelo $\mathrm{V}_{\mathrm{ZTC}}$ para transistores CMOS convencionais - Prijic $^{22}$}

Através dos modelos analíticos, muito bem conhecidos para a corrente de dreno (IDS) em transistores MOS convencionais ${ }^{14,15}$, e o modelo de aproximação da camada de inversão forte ${ }^{14}$, obtém-se ${ }^{22}$ : 


$$
\mathrm{I}_{\mathrm{DS}}=\mu_{\mathrm{eff}} \cdot \mathrm{C}_{\mathrm{ox}} \cdot \frac{\mathrm{W}}{\mathrm{L}}\left[\frac{\left(\mathrm{V}_{\mathrm{GS}}-\mathrm{V}_{\mathrm{th}}\right)^{2}}{2(1+\delta)}\right]\left(1+\rho^{2}\right)
$$

onde

$$
\rho= \begin{cases}1-\frac{V_{D S}}{\frac{V_{G S}-V_{t h}}{1+\delta}}, & V_{D S} \leq \frac{V_{G S}-V_{t h}}{1+\delta}(\text { região linear }) \\ 0 \quad, & V_{D S}>\frac{V_{G S}-V_{\text {th }}}{1+\delta} \text { (região de saturação) }\end{cases}
$$

e $\delta$ representa o fator de contribuição da tensão de dreno $\left(\mathrm{V}_{\mathrm{DS}}\right)$ sobre a largura da camada de depleção.

A dependência da mobilidade efetiva dos elétrons com a variação de temperatura pode ser assumida na forma da equação $(23)^{14,15}$.

Através das equações (25) e (26), obtém-se as expressões para a tensão de porta no ponto ZTC $\left(\mathrm{V}_{\mathrm{ZTC}}\right)$ para as regiões linear e de saturação ${ }^{22}$ :

Para a região linear tem-se:

$$
\mathrm{V}_{\mathrm{GS}}(\mathrm{ZTC})-\mathrm{V}_{\mathrm{th}}=-\frac{\mathrm{T}}{\mathrm{k}}\left(\frac{\mathrm{dV}_{\mathrm{th}}}{\mathrm{dT}}+\frac{1}{2} \mathrm{~V}_{\mathrm{DS}} \frac{\mathrm{d} \delta}{\mathrm{dT}}\right)+\frac{1}{2} \mathrm{~V}_{\mathrm{DS}}(1+\delta)
$$


E para a região de saturação será:

$$
\mathrm{V}_{\mathrm{GS}}(\mathrm{ZTC})-\mathrm{V}_{\mathrm{th}}=-\frac{2 \mathrm{~T}(1+\delta) \frac{\mathrm{dV} \text { th }}{\mathrm{dT}}}{(1+\delta) \mathrm{k}+\mathrm{T} \frac{\mathrm{d} \delta}{\mathrm{dT}}}
$$

Através da aplicação de alguns procedimentos ${ }^{18}$, para a minimização da diferença entre os dois lados da igualdade das equações (28) e (29) ${ }^{22}$, pode- se obter um ponto ideal de polarização da tensão de porta ( $\mathrm{V}_{\mathrm{GS}} \mathrm{ZTC}$ ), que garanta uma corrente de dreno sem variação com a temperatura.

\subsubsection{Modelo $\mathrm{V}_{\text {ZTC }}$ para MOS Convencionais - Shoucair ${ }^{16}$}

Para transistores MOSFETs com canal $\mathrm{N}$ ou $\mathrm{P}$, a condição que deve manter a corrente de dreno no ponto ZTC, $\mathrm{I}_{\mathrm{D}}(\mathrm{ZTC})^{16}$, é:

$$
\frac{1}{I_{D S}} \frac{d I_{D S}}{d T}=0=\frac{1}{\mu_{\text {eff }}(T)} \frac{d \mu_{\text {eff }}(T)}{d T}-\left[\frac{\mathrm{m}}{V_{G S}-V_{t h}(T)}\right] \frac{\mathrm{dV}_{\text {th }}(T)}{d T}
$$

onde $m=1$ na região linear, $m=2$ na região de saturação e $\mu_{\text {eff }}$ é obtido através da equação (31).

$$
\mu_{\mathrm{eff}}(\mathrm{T})=\mu_{\mathrm{eff}}\left(\mathrm{T}_{0}\right)\left(\frac{\mathrm{T}}{\mathrm{T}_{0}}\right)^{-1.5}
$$


Resolvendo-se a derivada da equação (30) tem-se:

$$
\mathrm{I}_{\mathrm{DS}}(\mathrm{ZTC}) \approx \frac{1}{2} \mu_{\mathrm{eff}}\left(\mathrm{T}_{0}\right) \mathrm{C}_{\mathrm{ox}} \frac{\mathrm{W}}{\mathrm{L}}\left[\frac{-\mathrm{m}}{1.5} \mathrm{~T}_{0} \frac{\mathrm{dV}_{\mathrm{th}}(\mathrm{T})}{\mathrm{dT}}\right]^{\mathrm{m}}
$$

onde

$$
\frac{\mathrm{dV}_{\mathrm{th}}(\mathrm{T})}{\mathrm{dT}}=\left[2+\frac{\gamma}{\left.\sqrt{\left[\mathrm{V}_{\mathrm{SB}}+2 \phi_{\mathrm{F}}(\mathrm{T})\right]}\right]}\left[\frac{\phi_{\mathrm{F}}(\mathrm{T})}{\mathrm{T}}-\frac{\mathrm{k}}{\mathrm{q}}\left(\frac{3}{2}+\frac{\mathrm{E}_{\mathrm{g} 0}}{2 \mathrm{qT}}\right)\right]\right.
$$

$V_{\mathrm{SB}}$ é a tensão de polarização do substrato, $\gamma$ é o fator de efeito de corpo independente da temperatura, $\mathrm{E}_{\mathrm{g} 0} \approx 1.2 \mathrm{eV}$ é a largura da faixa proibida do silício a $0^{\circ} \mathrm{K}$.

Para propostas de projeto de circuito, é desejável uma expressão explícita para a tensão de porta que polariza um transistor MOS no ponto ZTC ( $\mathrm{I}_{\mathrm{D}}(\mathrm{ZTC})$ ), que pode ser obtida analiticamente pelo método dos mínimos quadrados, minimizando o desvio do termo $\left[\mathrm{V}_{\mathrm{GS}}-\mathrm{V}_{\mathrm{th}}(\mathrm{T})\right.$ ] do valor determinado pela equação (30) sob uma faixa de temperatura especifica [T1, T2], em que T2 > T1. Contudo, a integração exigida para a resolução é complexa e o resultado exato não é de fácil tratamento matemático para obtenção de uma expressão simplificada. Desta forma obtem-se uma expressão derivada de aproximações com as equações (30) e (33) ${ }^{16}$ : 


$$
\begin{aligned}
\mathrm{V}_{\mathrm{GS}}(\mathrm{ZTC}) & \approx \frac{-\mathrm{p}_{0}}{6}(2 \mathrm{~m}-3)(\mathrm{T} 1+\mathrm{T} 2)+\mathrm{q}_{0}+ \\
& +\gamma\left\{\left[\sqrt{\mathrm{V}_{\mathrm{SB}}+2 \phi_{\mathrm{F}}\left(\mathrm{T}_{\mathrm{MED}}\right)}\right]-\sqrt{2 \phi_{\mathrm{F}}\left(\mathrm{T}_{\mathrm{MED}}\right)}\right\}- \\
& -\frac{\mathrm{m} \gamma}{1.5}\left[\frac{1}{\sqrt{\left[\mathrm{V}_{\mathrm{SB}}+2 \phi_{\mathrm{F}}\left(\mathrm{T}_{\mathrm{MED}}\right)\right]}}-\frac{1}{\left.\sqrt{\left[2 \phi_{\mathrm{F}}\left(\mathrm{T}_{\mathrm{MED}}\right)\right.}\right]}\right] \times\left[\phi_{\mathrm{F}}\left(\mathrm{T}_{\mathrm{MED}}\right)-\frac{\mathrm{E}_{\mathrm{G} 0}}{2 \mathrm{q}}\right]
\end{aligned}
$$

onde $p_{0}=d V_{t h} / d T, q_{0} \approx V_{\text {th }}\left(T_{0}\right)-p_{0} T_{0}, T_{0}$ é a temperatura de referência e $\phi_{F}\left(T_{M E D}\right)$ é a média do valor do potencial de Fermi sobre a faixa de temperatura [T1, T2] .

\subsubsection{Modelo $\mathrm{V}_{\text {ZTC }}$ para SOI MOSFETs Parcialmente Depletados -} Osman $^{21}$

O modelo ${ }^{53}$ reportado para corrente de dreno de dispositivos SOI MOSFET Parcialmente Depletados (PD-SOI) para ambas regiões de operação é:

Região Linear:

$$
\mathrm{I}_{\mathrm{DS}}(\mathrm{T})=\frac{\mathrm{W}}{\mathrm{L}} \mu_{\text {eff }}(\mathrm{T}) \cdot \mathrm{C}_{\mathrm{oxf}} \cdot\left[\left(\mathrm{V}_{\mathrm{GF}}-\mathrm{V}_{\text {thF }}(\mathrm{T})\right) \mathrm{V}_{\mathrm{DS}}-\frac{1+\alpha(\mathrm{T})}{2} \mathrm{~V}_{\mathrm{DS}}{ }^{2}\right]
$$

Região de Saturação:

$$
\mathrm{I}_{\mathrm{DS}}(\mathrm{T})=\frac{\mathrm{W}}{2 \mathrm{~L}} \mu_{\mathrm{eff}}(\mathrm{T}) \cdot \mathrm{C}_{\mathrm{oxf}} \cdot \frac{\left(\mathrm{V}_{\mathrm{GF}}-\mathrm{V}_{\mathrm{thF}}(\mathrm{T})\right)^{2}}{(1+\alpha(\mathrm{T}))}
$$


onde T é a temperatura, W e L são a largura e comprimento do canal, $\mathrm{C}_{\text {oxf }}$ é a capacitância do óxido de porta e $(1+\alpha)$ é termo do fator de efeito de corpo. $\mathrm{V}_{\mathrm{GF}} \mathrm{e} \mathrm{V}_{\text {thF }}$ são a tensão aplicada a porta e a tensão de limiar, respectivamente, enquanto que $\mu_{\text {eff }}$ representa a mobilidade dos portadores que é modelada ${ }^{54}$ por:

$$
\mu_{\text {eff }}(T)=\frac{\mu_{0}(T)}{1+\theta(T)\left[V_{G F}-V_{\text {thF }}(T)\right]}
$$

O parâmetro $\theta(T)$ dimensiona a degradação da mobilidade com o campo elétrico transversal e o parâmetro $\mu_{0}(T)$ é a mobilidade de baixo campo que tem uma dependência com a temperatura na forma da equação (23).

Através das equações (35) e (36) e da definição do ponto ZTC pela equação (25), pode-se demonstrar que a polarização da porta no ponto ZTC é dada por:

$$
\mathrm{V}_{\mathrm{GF}}(\mathrm{zTC})=\mathrm{V}_{\mathrm{thF}}+\frac{-\mathrm{B}_{\mathrm{Os}}+\sqrt{\mathrm{B}_{\mathrm{Os}}{ }^{2}+4 \mathrm{~A}_{\mathrm{Os}} \mathrm{C}_{\mathrm{Os}}}}{2 \mathrm{~A}_{\mathrm{Os}}}
$$

onde $\mathrm{A}_{\mathrm{O} s}, \mathrm{~B}_{\mathrm{O}}$ e $\mathrm{C}_{\mathrm{Os}}$ são determinados no ponto ZTC de acordo com qual regime está se operando, linear ou de saturação. As aproximações usadas na equação acima são as mesmas que adotadas no modelo de Prijic $^{22}$ para tecnologia CMOS convencional. Na região linear, os parâmetros $A_{O s}$, $B_{O s} e$ $\mathrm{C}_{\text {Os }}$ da equação (38) são definidos como ${ }^{21}$ : 


$$
\begin{aligned}
& A_{\mathrm{Os}}=\frac{\mathrm{c}}{\mathrm{T}} \theta(\mathrm{T})-\frac{\mathrm{d} \theta(\mathrm{T})}{\mathrm{dT}} \\
& \mathrm{B}_{\mathrm{Os}}=\frac{\mathrm{c}}{\mathrm{T}}\left(1-\frac{\mathrm{V}_{\mathrm{DS}} \theta(\mathrm{T})}{2}(1+\alpha(\mathrm{T}))\right)+(1+\alpha(\mathrm{T})) \mathrm{V}_{\mathrm{DS}} \frac{\mathrm{d} \theta(\mathrm{T})}{\mathrm{dT}} \\
&+\theta(\mathrm{T}) \frac{\mathrm{dV} \text { thF }}{\mathrm{dT}}+\theta(\mathrm{T})\left(\frac{\mathrm{dV} \mathrm{V}_{\mathrm{thF}}(\mathrm{T})}{\mathrm{dT}}+\frac{1}{2} \frac{\mathrm{d} \alpha(\mathrm{T})}{\mathrm{dT}} \mathrm{V}_{\mathrm{DS}}\right) \\
& \mathrm{C}_{\mathrm{Os}}=\frac{\mathrm{c}}{\mathrm{T}} \mathrm{V}_{\mathrm{DS}}(1+\alpha(\mathrm{T}))+\mathrm{V}_{\mathrm{DS}} \theta(\mathrm{T})(1+\alpha(\mathrm{T})) \frac{\mathrm{dV} \text { thF }}{\mathrm{dT}}(\mathrm{T}) \\
&-\left(\frac{\mathrm{dV} \mathrm{V}_{\mathrm{thF}}(\mathrm{T})}{\mathrm{dT}}+\frac{\mathrm{d} \alpha(\mathrm{T})}{2 \mathrm{dT}} \mathrm{V}_{\mathrm{DS}}\right)
\end{aligned}
$$

Por outro lado, se o ponto ZTC está na região de saturação, então os parâmetros $\mathrm{A}_{\mathrm{Os}}, \mathrm{B}_{\mathrm{Os}}$ e $\mathrm{C}_{\mathrm{Os}}$ serão dadas por:

$$
\begin{aligned}
& \mathrm{A}_{\mathrm{Os}}=\theta(\mathrm{T})\left(\frac{\mathrm{c}}{\mathrm{T}}+\frac{\mathrm{d} \alpha(\mathrm{T})}{\mathrm{dT}}\right)+\frac{\mathrm{d} \theta(\mathrm{T})}{\mathrm{dT}}(1+\alpha(\mathrm{T})) \\
& \mathrm{B}_{\mathrm{Os}}=\frac{\mathrm{c}}{\mathrm{T}}+\frac{\mathrm{d} \alpha(\mathrm{T})}{\mathrm{dT}}+2 \frac{\mathrm{dV}_{\mathrm{thF}}(\mathrm{T})}{\mathrm{dT}} \theta(\mathrm{T})-\theta(\mathrm{T}) \frac{\mathrm{dV}_{\mathrm{thF}}(\mathrm{T})}{\mathrm{dT}}(1+\alpha(\mathrm{T})) \\
& \mathrm{C}_{\mathrm{Os}}=2 \frac{\mathrm{dV}_{\mathrm{thF}}(\mathrm{T})}{\mathrm{dT}}
\end{aligned}
$$

O valor ótimo da tensão de polarização de porta no ponto ZTC, $\mathrm{V}_{\mathrm{GS}}(\mathrm{ZTC})$, é obtido pela diminuição da diferença entre os lados da equação (38) através do método dos mínimos quadrados sob a faixa de temperatura investigada $\left[27,300^{\circ} \mathrm{C}\right]$. 


\begin{abstract}
Os modelos apresentados ${ }^{16,21,22}$ analisam e demonstram a modelagem para a polarização no ponto ZTC para dispositivos MOS convencionais e SOI MOSFETs parcialmente depletados. Estes trabalhos possuem propostas de determinação para a polarização no ponto ZTC, que apesar das aproximações e simplificações que foram adotadas, utilizam expressões extensas que podem ser bastante complexas, ou seja, em uma análise inicial não é possível perceber como a variação dos principais parâmetros do transistor irão influênciar no valor de $V_{\text {ZTC. Estes modelos }}$ garantem uma alta precisão e um equacionamento muito aplicável para uso nos modelos inseridos em programas simuladores numéricos e analíticos disponíveis comercialmente.
\end{abstract}

No próximo capítulo será apresentada uma proposta de modelo simples desenvolvido para a avaliação do ponto ZTC em transistores SOI MOSFETs parcialmente (PD) e totalmente (FD) depletados, sendo que este último é inédito. A idéia de um modelo simples proporciona condições de análise dos principais fatores que influênciam na obtenção do ponto ZTC, desta forma permitindo uma percepção direta de como cada fator o afeta. 


\section{Proposta de um Modelo Analítico Simples para determinação da tensão de polarização no ponto invariante com a temperatura $\left(\mathrm{V}_{\mathrm{ZTC}}\right)$}

Neste capítulo é apresentada uma proposta de um modelo analítico simples para estudo do comportamento da tensão de porta $\left(\mathrm{V}_{\mathrm{GF}}\right)$ igual a tensão no ponto invariante com a temperatura $\left(\mathrm{V}_{\mathrm{ZTC}}\right)$, operando nas regiões linear e de saturação. A validação do modelo apresentado é realizada através da comparação dos resultados das simulações do modelo $\mathrm{CM}$ proposto e os dados experimentais.

\subsection{Motivação da proposta do modelo analítico simples do ponto invariante com a temperatura (ZTC)}

A tecnologia SOI CMOS tem sido amplamente utilizada em aplicações de alta temperatura devido a características como o baixo valor das correntes de fuga, o melhor fator de inclinação de sublimiar e a supressão do fenômeno de tiristor parasitário ("latch-up") comparado com os dispositivos de tecnologia MOS convencional ${ }^{26,48}$.

Como descrito no capítulo 3 , a determinação da tensão no ponto de polarização invariante com a temperatura $\left(\mathrm{V}_{\mathrm{ZTC}}\right)$ é muito importante para a estabilidade de circuitos que operam em uma larga faixa de valores de temperatura. Portanto, foi verificada a existência de trabalhos, através de propostas de modelos analíticos, a respeito do comportamento dos transistores com tecnologia MOS no ponto ZTC. 
Esses trabalhos foram realizados para transistores MOS convencionais $^{16,22}$ e para dispositivos SOI MOSFET em regime parcialmente depletado (PD-SOI) ${ }^{21}$, com destaque para análise de sua influência sobre parâmetros importantes como a dependência da tensão de limiar $\left(\mathrm{V}_{\mathrm{thF}}\right)$ e da mobilidade dos elétrons $\left(\mu_{n}\right)^{16}$ com a temperatura, o efeito do fator de corpo $(n)^{22}$ e o fator de degradação da mobilidade devido ao campo elétrico transversal $(\theta)^{21}$.

Os trabalhos supracitados já apresentam uma modelagem para a polarização no ponto ZTC de transistores com tecnologias MOS convencional e SOI MOSFET parcialmente depletados, entretanto estes trabalhos desenvolvem expressões que, mesmo com aproximações, incluem alguns termos que são derivados de outras expressões extremamente extensas. Desta forma garantem a alta precisão e um equacionamento muito aplicável para uso em modelos que são inseridos nos programas de simulação numérica e analítica disponíveis comercialmente, mas não permitem uma análise mais direta e simples da influência dos principais parâmetros no comportamento dos transistores no ponto ZTC.

A seguir será apresentado o desenvolvimento de um modelo simples para a avaliação do ponto ZTC, em transistores SOI MOSFETs parcialmente (PD) e totalmente (FD) depletados, permitindo a análise dos principais fatores que influênciam na obtenção deste ponto.

\subsubsection{Modelo Camillo-Martino (Modelo $\mathrm{CM}$ ): Modelo de $\mathrm{V}_{\mathrm{ZTC}}$ na Região Linear}

Na região linear, a corrente de dreno ( $\left.I_{D S}\right)$ de um SOI MOSFET, em regime de inversão forte, pode ser calculada usando-se a equação $(41)^{53}$ na temperatura T1: 


$$
\mathrm{I}_{\mathrm{DS} 1}=\mu_{\mathrm{n} 1} \cdot \mathrm{C}_{\mathrm{oxf}} \cdot \frac{\mathrm{W}}{\mathrm{L}}\left[\left(\mathrm{V}_{\mathrm{GF}}-\mathrm{V}_{\mathrm{th} 1}\right) \mathrm{V}_{\mathrm{DS}}-\frac{\mathrm{n}_{1} \mathrm{~V}_{\mathrm{DS}}^{2}}{2}\right]
$$

onde $\mathrm{C}_{\mathrm{oxf}}=\varepsilon_{\mathrm{ox}} / \mathrm{t}_{\mathrm{ox}}, \mathrm{t}_{\mathrm{ox}}$ é a espessura do óxido de porta, $\varepsilon_{\mathrm{ox}}$ é a permissividade do dióxido de silício $\left(\mathrm{SiO}_{2}\right)$, W e $\mathrm{L}$ são a largura e comprimento do canal do transistor, respectivamente, $V_{G F}$ e $V_{D S}$ são as tensões aplicadas aos terminais de porta e dreno, respectivamente em relação ao terminal de fonte. Os parâmetros $\mu_{\mathrm{n} 1}, V_{\text {th1 }}$ e $\mathrm{n}_{1}$ são a mobilidade efetiva dos elétrons, tensão de limiar e fator de efeito de corpo, respectivamente, para o caso da temperatura igual a T1.

O parâmetro $\mu_{\mathrm{n} 1}$ é definido em função da mobilidade de baixo campo $\left(\mu_{\mathrm{n} 0(\mathrm{~T} 1)}\right)$, o fator de degradação da mobilidade $(\theta)$, tensão aplicada à porta do transistor $\left(\mathrm{V}_{\mathrm{GF}}\right)$ e a tensão de limiar $\left(\mathrm{V}_{\mathrm{th}}\right)$, como $^{55}$ :

$$
\mu_{\mathrm{n} 1}=\frac{\mu_{\mathrm{n} 0(\mathrm{~T} 1)}}{1+\frac{\theta_{1}}{\mathrm{n}_{1}}\left(\mathrm{~V}_{\mathrm{GF}}-\mathrm{V}_{\mathrm{th} 1}\right)}
$$

Por definição, o ponto de polarização ZTC $\left(\mathrm{V}_{\mathrm{ZTC}}\right)$ representa a tensão de porta que garante que a corrente de dreno permaneça constante com a variação de temperatura. Através da equação (41) e na temperatura T2 (T2 > T1), obtém-se o ponto de cruzamento entre as curvas da corrente de dreno $I_{D S}$ por $V_{G F}$, que apresenta as coordenadas $V_{Z T C}(T 1, T 2)$ e $I_{Z T C}(T 1, T 2)$, como mostrado na Figura 11. 


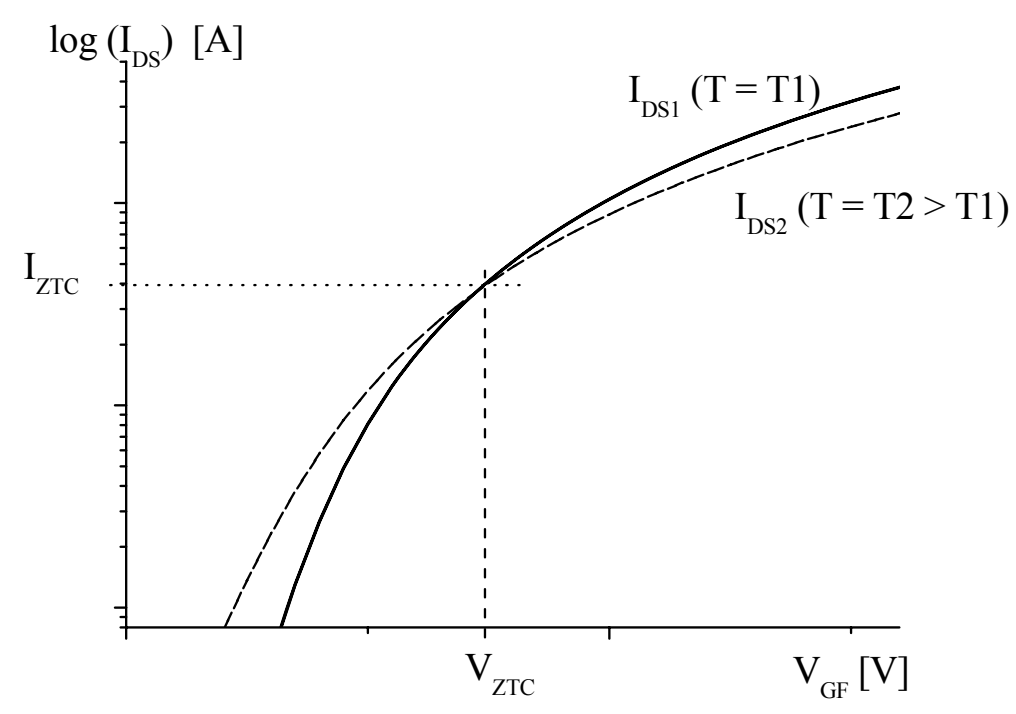

Figura 11: $\mathrm{O}$ ponto $Z T C$ na curva $\mathrm{I}_{\mathrm{DS}} \times \mathrm{V}_{\mathrm{GF}}$ para temperaturas $\mathrm{T} 1 \mathrm{e} \mathrm{T} 2$.

Admitindo-se que o ponto ZTC na tensão de polarização de porta ocorre na região linear (triodo $\mathrm{V}_{\mathrm{ZTC}}>\mathrm{V}_{\mathrm{thF}}+\mathrm{V}_{\mathrm{DS}}$ ), tem-se para a corrente de dreno ${ }^{55}$ :

$$
\begin{aligned}
& \mathrm{I}_{\mathrm{DS} 1}=\frac{\mu_{\mathrm{n} 0(\mathrm{~T} 1)}}{1+\frac{\theta_{1}}{\mathrm{n}_{1}}\left(\mathrm{~V}_{\mathrm{GF}}-\mathrm{V}_{\mathrm{th} 1}\right)} \mathrm{C}_{\mathrm{oxf}} \frac{\mathrm{W}}{\mathrm{L}}\left[\left(\mathrm{V}_{\mathrm{GF}}-\mathrm{V}_{\mathrm{th} 1}\right) \mathrm{V}_{\mathrm{DS}}-\mathrm{n}_{1} \frac{\mathrm{V}_{\mathrm{DS}}^{2}}{2}\right] \\
& \mathrm{I}_{\mathrm{DS} 2}=\frac{\mu_{\mathrm{n} 0(\mathrm{~T} 2)}}{1+\frac{\theta_{2}}{\mathrm{n}_{2}}\left(\mathrm{~V}_{\mathrm{GF}}-\mathrm{V}_{\mathrm{th} 2}\right)} \mathrm{C}_{\mathrm{oxf}} \frac{\mathrm{W}}{\mathrm{L}}\left[\left(\mathrm{V}_{\mathrm{GF}}-\mathrm{V}_{\mathrm{th} 2}\right) \mathrm{V}_{\mathrm{DS}}-\mathrm{n}_{2} \frac{\mathrm{V}_{\mathrm{DS}}^{2}}{2}\right]
\end{aligned}
$$

Para $V_{G F}=V_{Z T C}$ implica em $I_{D S 1}=I_{D S 2}$, e considerando que em uma primeira análise, a variação com a temperatura dos fatores de efeito de corpo e de degradação da mobilidade pode ser aproximada para $n_{1} \simeq n_{2} \simeq n$ e $\theta_{1} \simeq \theta_{2} \simeq \theta$, respectivamente, desta forma pode-se também aproximar: 


$$
\frac{\theta_{1}}{n_{1}} \approx \frac{\theta_{2}}{n_{2}} \quad \text { e } \quad 1+\frac{\theta_{1}}{\mathrm{n}_{1}}\left(\mathrm{~V}_{\mathrm{GF}}-\mathrm{V}_{\mathrm{th} 1}\right) \approx 1+\frac{\theta_{2}}{\mathrm{n}_{2}}\left(\mathrm{~V}_{\mathrm{GF}}-\mathrm{V}_{\mathrm{th} 2}\right)
$$

Com as considerações adotadas, a tensão $V_{\text {ZTC }}$ pode ser isolada como mostrada na equação (45).

$$
\mathrm{V}_{\mathrm{ZTC}}(\mathrm{T} 1, \mathrm{~T} 2)_{\mathrm{LIN}}=\mathrm{V}_{\mathrm{ZTCLIN}}=\frac{\mu_{\mathrm{n} 1} \cdot \mathrm{V}_{\mathrm{th} 1}-\mu_{\mathrm{n} 2} \cdot \mathrm{V}_{\mathrm{th} 2}}{\mu_{\mathrm{n} 1}-\mu_{\mathrm{n} 2}}+\mathrm{n} \frac{\mathrm{V}_{\mathrm{DS}}}{2}
$$

onde $\mu_{\mathrm{n} 1}$ e $\mu_{\mathrm{n} 2}$ são as mobilidades efetivas dos elétrons nas temperaturas T1 e T2, respectivamente, e $n$ é o efeito de corpo de acordo com estrutura analisada, equação (10).

Utilizando a relação que mostra a variação da mobilidade efetiva dos elétrons com a temperatura ${ }^{22}$ :

$$
\mu_{\mathrm{n} 2}=\mu_{\mathrm{n} 1}\left(\frac{\mathrm{T} 1}{\mathrm{~T} 2}\right)^{c}
$$

e substituindo (46) em (45), pode-se obter a equação (47) com algumas simplificações, que representa o valor da tensão de polarização no ponto ZTC.

$$
\mathrm{V}_{\mathrm{ZTC} \mathrm{LIN}}=\mathrm{V}_{\mathrm{th} 1}+\frac{\left(\mathrm{V}_{\mathrm{th} 1}-\mathrm{V}_{\mathrm{th} 2}\right)\left(\frac{\mathrm{T} 1}{\mathrm{~T} 2}\right)^{\mathrm{c}}}{1-\left(\frac{\mathrm{T} 1}{\mathrm{~T} 2}\right)^{\mathrm{c}}}+\mathrm{n} \frac{\mathrm{V}_{\mathrm{DS}}}{2}
$$




\subsubsection{Modelo Camillo-Martino (Modelo $\mathrm{CM}$ ): Modelo de $\mathrm{V}_{\mathrm{zTC}}$ na Região de Saturação}

$\mathrm{Na}$ região de saturação, a corrente de dreno (lDS) em regime de inversão forte de um SOI MOSFET pode ser calculada, usando-se a equação $(48)^{53}$ na temperatura T1:

$$
\mathrm{I}_{\mathrm{DS} 1}=\mu_{\mathrm{n} 1} \cdot \mathrm{C}_{\mathrm{oxf}} \cdot \frac{\mathrm{W}}{\mathrm{L}}\left[\frac{\left(\mathrm{V}_{\mathrm{GF}}-\mathrm{V}_{\mathrm{th} 1}\right)^{2}}{2 \mathrm{n}_{1}}\right]
$$

onde $\mathrm{C}_{\text {oxf }}=\varepsilon_{\mathrm{ox}} / \mathrm{t}_{\mathrm{oxf}}$, $\mathrm{t}_{\mathrm{oxf}}$ é a espessura do óxido de porta, $\varepsilon_{\mathrm{ox}}$ é a permissividade do dióxido de silício $\left(\mathrm{SiO}_{2}\right), W$ e $\mathrm{L}$ são a largura e comprimento do canal do transistor, respectivamente, e $V_{G F}$ é a tensão aplicada ao terminal de porta. Os parâmetros $\mu_{\mathrm{n} 1}, \mathrm{~V}_{\mathrm{th} 1}$ e $\mathrm{n}_{1}$ são a mobilidade efetiva dos elétrons, tensão de limiar e fator de efeito de corpo, respectivamente, caso a temperatura seja igual a T1.

Através da definição do ponto de polarização ZTC ( $\mathrm{V}_{\text {ZTC }}$ ) aplicada à equação (47) e considerando a temperatura T2 (T2 > T1), obtém-se o ponto de cruzamento entre as curvas da corrente de dreno $I_{D S}$ por $V_{G S}$ que apresenta as coordenadas $\mathrm{V}_{\mathrm{ZTC}}(\mathrm{T} 1, \mathrm{~T} 2)$ e $\mathrm{I}_{\mathrm{ZTC}}(\mathrm{T} 1, \mathrm{~T} 2)$, como mostrado na Figura 11.

Admitindo-se que o ponto ZTC na tensão de polarização de porta ocorre na região de saturação, tem-se para a corrente de dreno:

$$
\mathrm{I}_{\mathrm{DS} 1}=\frac{\mu_{\mathrm{n} 1}}{1+\frac{\theta_{1}}{\mathrm{n}_{1}}\left(\mathrm{~V}_{\mathrm{GF}}-\mathrm{V}_{\mathrm{th} 1}\right)} \mathrm{C}_{\mathrm{oxf}} \frac{\mathrm{W}}{\mathrm{L}} \frac{\left(\mathrm{V}_{\mathrm{GF}}-\mathrm{V}_{\mathrm{th} 1}\right)^{2}}{2 \mathrm{n}_{1}}
$$




$$
\mathrm{I}_{\mathrm{DS} 2}=\frac{\mu_{\mathrm{n} 2}}{1+\frac{\theta_{2}}{\mathrm{n}_{2}}\left(\mathrm{~V}_{\mathrm{GF}}-\mathrm{V}_{\mathrm{th} 2}\right)} \mathrm{C}_{\mathrm{oxf}} \frac{\mathrm{W}}{\mathrm{L}} \frac{\left(\mathrm{V}_{\mathrm{GF}}-\mathrm{V}_{\mathrm{th} 2}\right)^{2}}{2 \mathrm{n}_{2}}
$$

Do mesmo modo que foi realizado no modelo em regime linear, para o modelo em regime de saturação, serão adotadas as aproximações para os parâmetros de fator de efeito de corpo e de degradação da mobilidade, ou seja, $n_{1} \simeq n_{2} \simeq \mathrm{n}$ e $\theta_{1} \simeq \theta_{2} \simeq \theta$, respectivamente, e a aproximação para o termo $1+\frac{\theta_{1}}{\mathrm{n}_{1}}\left(\mathrm{~V}_{\mathrm{GF}}-\mathrm{V}_{\text {th } 1}\right) \approx 1+\frac{\theta_{2}}{\mathrm{n}_{2}}\left(\mathrm{~V}_{\mathrm{GF}}-\mathrm{V}_{\text {th } 2}\right)$.

Resultando na equação (51) para a determinação da tensão $V_{\text {ZTC }}$ no regime de saturação.

$$
\mathrm{V}_{\mathrm{ZTC}}(\mathrm{T} 1, \mathrm{~T} 2)=\mathrm{V}_{\mathrm{ZTCSAT}}=\mathrm{A}+\sqrt{\mathrm{A}^{2}-\mathrm{B}}
$$

onde

$$
\mathrm{A}=\frac{\mu_{\mathrm{n} 1} \cdot \mathrm{V}_{\mathrm{th} 1}-\mu_{\mathrm{n} 2} \cdot \mathrm{V}_{\mathrm{th} 2}}{\mu_{\mathrm{n} 1}-\mu_{\mathrm{n} 2}}
$$

e

$$
\mathrm{B}=\frac{\mu_{\mathrm{n} 1} \cdot \mathrm{V}_{\mathrm{th} 1}^{2}-\mu_{\mathrm{n} 2} \cdot \mathrm{V}_{\mathrm{th} 2}^{2}}{\mu_{\mathrm{n} 1}-\mu_{\mathrm{n} 2}}
$$

Utilizando a relação que mostra a variação da mobilidade efetiva dos elétrons com a temperatura ${ }^{22}$ através da equação (46), e substituindo nas expressões (52) e (53): 


$$
\begin{aligned}
& A=V_{\text {th } 1}+\frac{\left(V_{\text {th } 1}-V_{\text {th } 2}\right)\left(\frac{T 1}{T 2}\right)^{c}}{1-\left(\frac{T 1}{T 2}\right)^{c}} \\
& B=\frac{V_{\text {th } 1}^{2}-V_{\text {th } 2}^{2}\left(\frac{T 1}{T 2}\right)^{c}}{1-\left(\frac{T 1}{T 2}\right)^{c}}
\end{aligned}
$$

Deste modo obtém-se o valor de $\mathrm{V}_{\text {ZTC }}$ dependente da tensão de limiar e do fator de degradação da mobilidade, ambos com relação a variação de temperatura de T1 para T2.

\subsection{Validação do Modelo CM}

O objetivo desta etapa é validar o modelo $\mathrm{CM}$ proposto, para a determinação da tensão de polarização do terminal de porta no ponto ZTC, através das equações (47) e (51) que consideram transistor operando nas regiões linear e de saturação, respectivamente.

A verificação será realizada através de comparação dos resultados simulados pelo modelo $\mathrm{CM}$ proposto e os dados experimentais.

\subsubsection{Características dos Dispositivos}

Os dispositivos SOI n e p MOSFETs parcialmente (PD) e totalmente (FD) depletados foram fabricados com tecnologia SOI CMOS $0.13 \mu \mathrm{m}$. No caso dos dispositivos PD-SOI, a lâmina foi obtida pela técnica UNIBOND com uma espessura de óxido enterrado de $400 \mathrm{~nm}$ e $200 \mathrm{~nm}$ para 
dispositivos FD-SOI. A estrutura de porta consiste de $2.5 \mathrm{~nm}$ de óxido-nitreto (NO) e um eletrodo com $150 \mathrm{~nm}$ de poli-silício. A técnica STI foi aplicada para o campo de isolação nos dispositivos FD-SOI e PELOX para os transistores PD-SOI. A espessura final da camada de silício é de $30 \mathrm{~nm}$ e 100nm para dispositivos FD e PD, respectivamente, Figura 12.

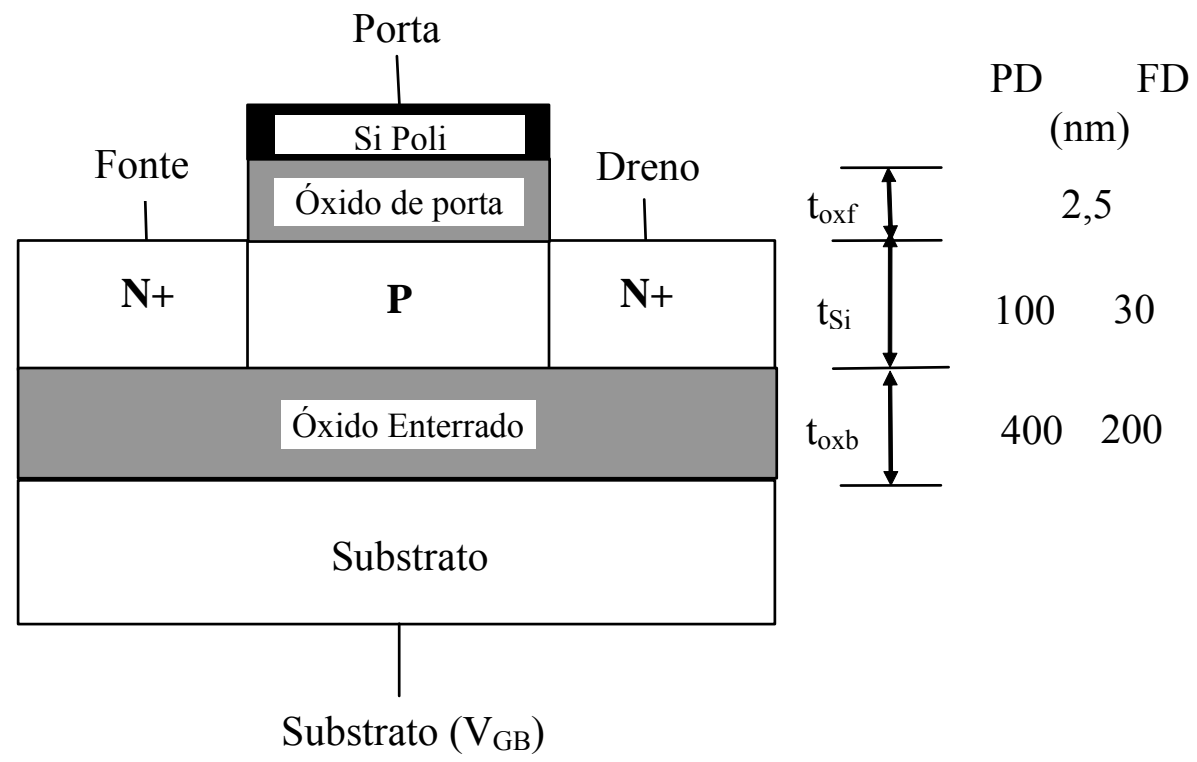

Figura 12 - Perfil do transistor nMOSFET SOI ilustrando as características dos dispositivos parcialmente e totalmente depletados.

\subsubsection{Resultados experimentais e modelo CM proposto para dispositivos SOI Convencionais}

Neste capítulo são apresentadas as curvas características dos dispositivo PD e FD SOI MOSFET em diferentes temperaturas, as quais foram obtidas experimentalmente utilizando os seguintes equipamentos: "Variable Temperature Micro Probe System", modelo K20 da MMR Technology; e o analisador de parâmetros Agilent - 4156C 


\subsubsection{Região Linear de Operação}

A Figura 13 e a Figura 14 apresentam as curvas $I_{D S} \times V_{G F}$ obtidas através de medidas experimentais com transistores SOI nMOSFETs parcialmente (PD) e totalmente (FD) depletados, em diferentes temperaturas e operando na região linear de polarização. É possível notar a ocorrência do ponto ZTC em ambos os tipos de dispositivos, obtendo o valor de $\mathrm{V}_{\mathrm{ZTC}} \simeq$ $0,47 \vee$ e $V_{\mathrm{ZTC}} \simeq 0,36 \mathrm{~V}$ para os transistores PD-SOI e FD-SOI, respectivamente.

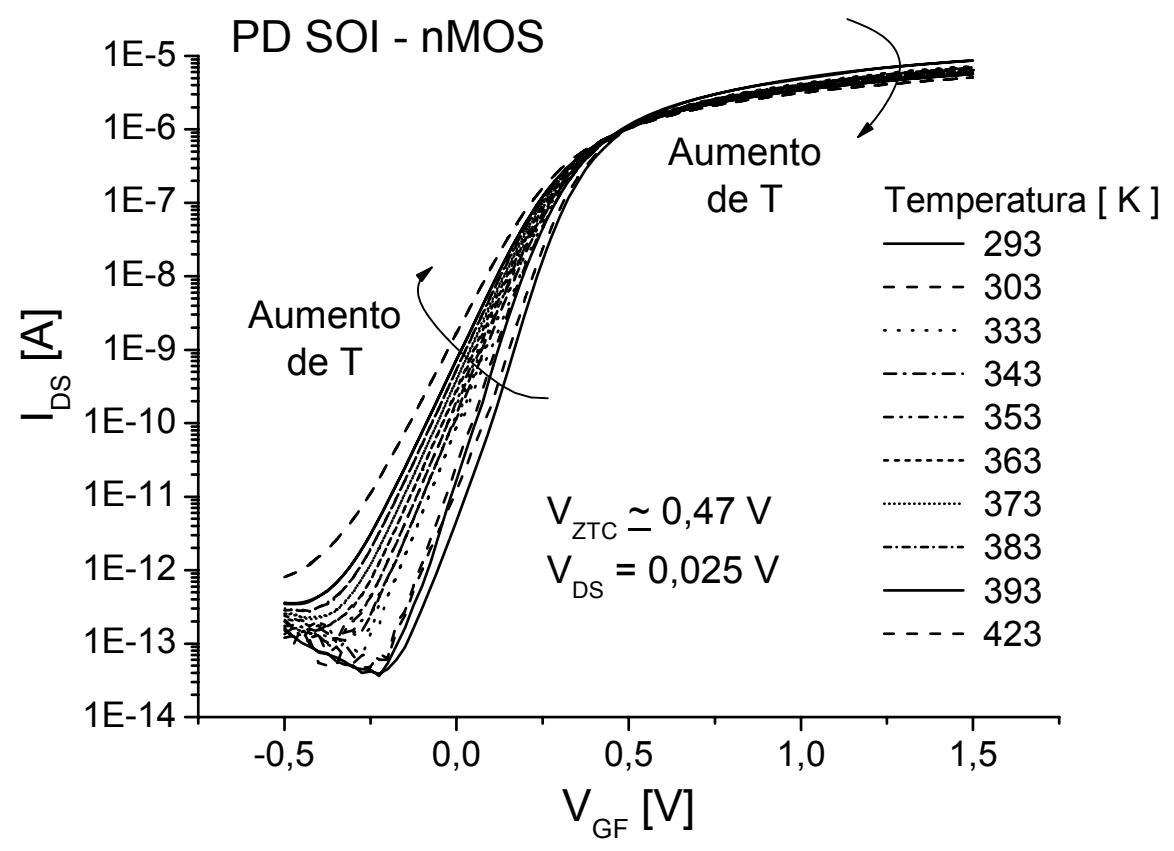

Figura 13: Curvas $I_{D S} \times V_{G F}$ de medidas experimentais do transistor PD-SOI nMOS em diferentes temperaturas. 


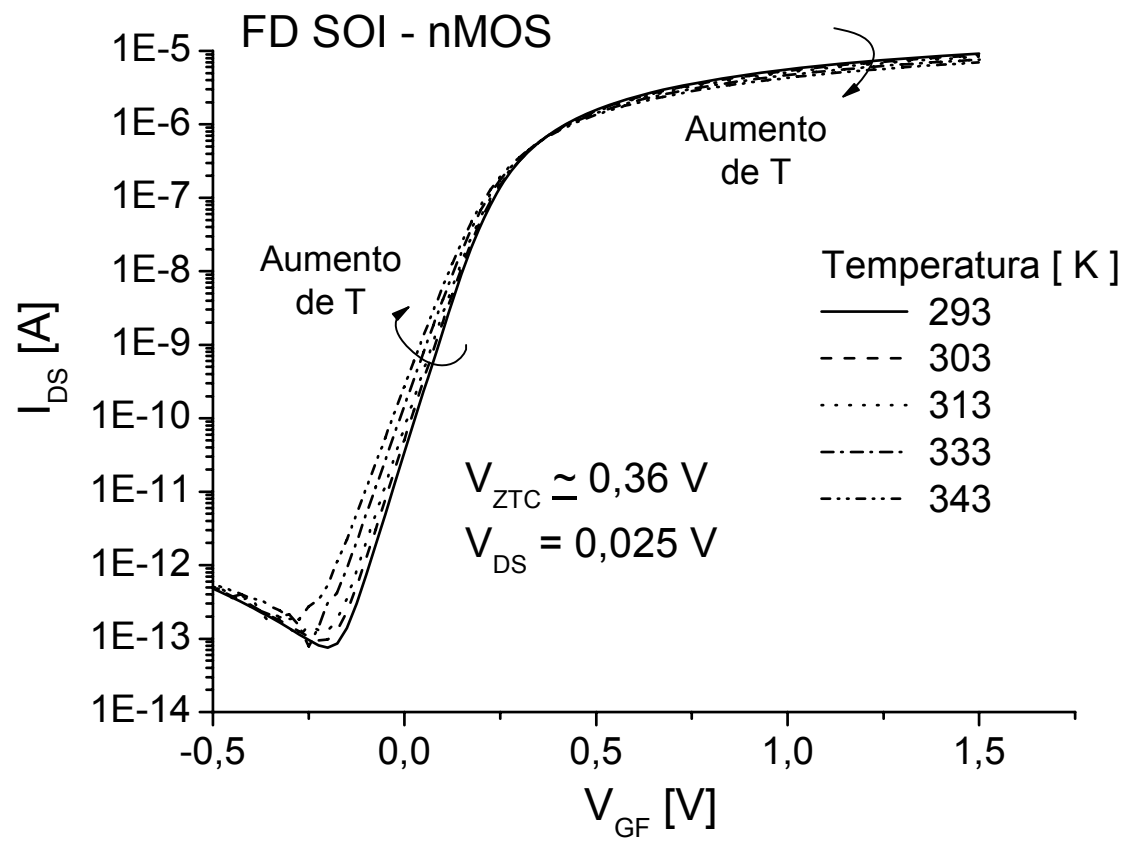

Figura 14: Curvas $I_{D S} \times V_{G F}$ de medidas experimentais do transistor FD-SOI nMOS em diferentes temperaturas.

As medidas experimentais ( $\left.I_{D S} \times V_{G F}\right)$ com transistores SOI pMOSFETs parcialmente (PD) e totalmente (FD) depletados, em diferentes temperaturas e operando na região linear de polarização são apresentadas na Figura 15 e Figura 16. Também é possível notar a ocorrência do ponto ZTC em ambos os tipos de dispositivos, obtendo o valor de $\mathrm{V}_{\mathrm{ZTC}} \simeq-0,8 \mathrm{Ve}$ $\mathrm{V}_{\mathrm{ZTC}} \simeq$ - 0,5 $\mathrm{V}$ para os transistores PD-SOI e FD-SOI, respectivamente. 


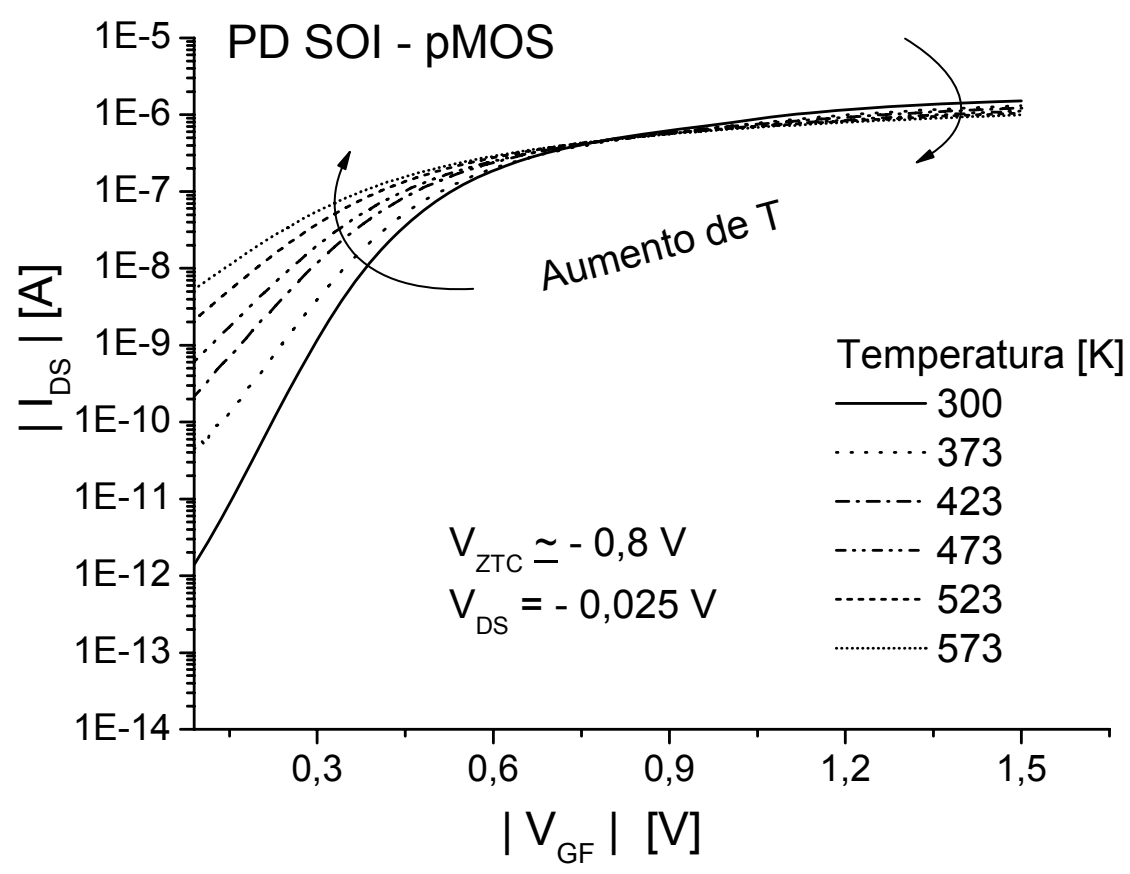

Figura 15: Curvas $I_{D S} \times V_{G F}$ de medidas experimentais do transistor PD-SOI pMOS em diferentes temperaturas.

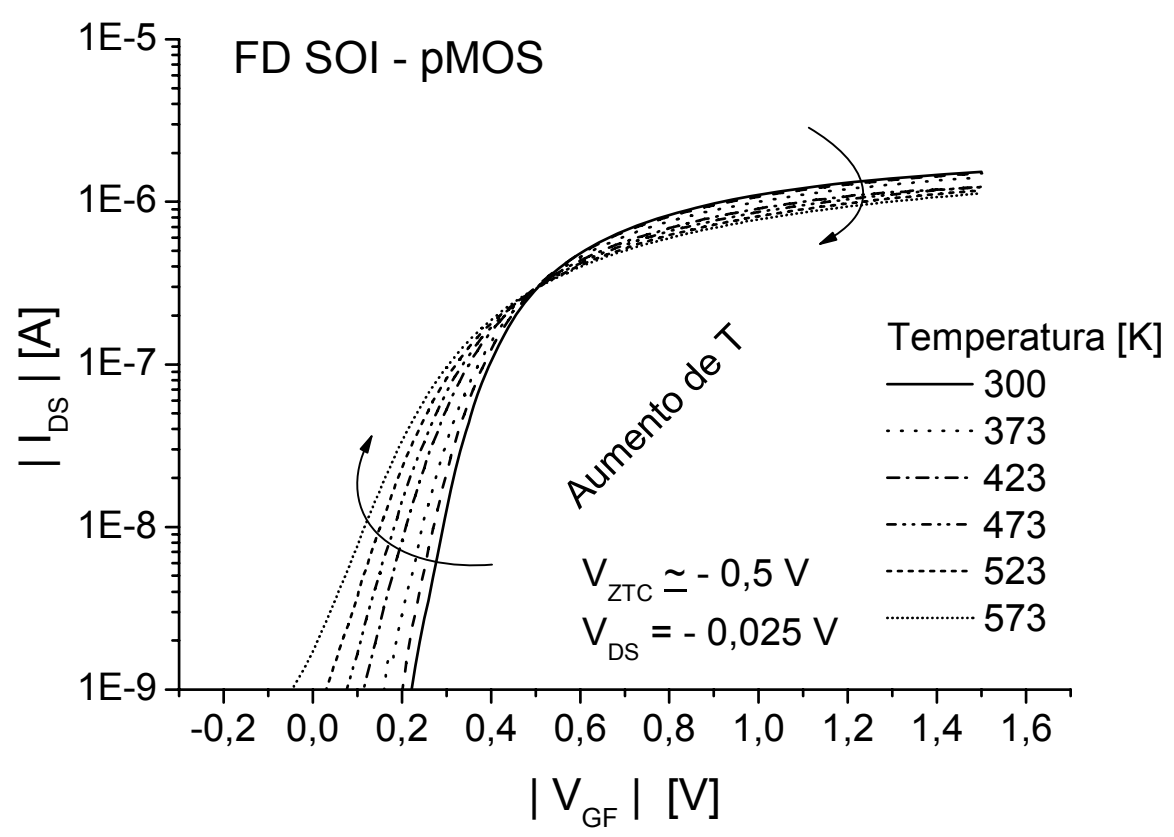

Figura 16: Curvas $I_{D S} \times V_{G F}$ de medidas experimentais do transistor FD-SOI pMOS em diferentes temperaturas. 
Da Figura 17 a Figura 20 é apresentado o valor de $V_{\text {ZTC }}$ em função da temperatura, para os dispositivos SOI n e p MOSFETs para os dois tipos PD e FD, que foi obtido experimentalmente e pelo modelo $\mathrm{CM}$ proposto operando na região linear através da equação (47), em que os valores das tensões de limiar $V_{\text {th1 }}$ e $\quad V_{\text {th2 }}$ inseridos são valores obtidos experimentalmente. $O$ valor obtido de $V_{\text {ZTC }}$ é o valor do ponto de cruzamento entre a curva $I_{D S} \times V_{G F}$ na temperatura de referencia $(T 1=300 \mathrm{~K})$ e as curvas $I_{D S} \times V_{G F}$ nas demais temperaturas analisadas (323 a 573K), portanto mostrando que $V_{\text {ZTC }}$ na prática configura-se como uma faixa de valores de $V_{G F}$ e não um ponto único, em que não há variação de $I_{D S}$ com a temperatura.

Os valores do fator $c$, que representa a degradação da mobilidade com a temperatura, utilizados para o ajuste dos resultados do modelo $\mathrm{CM}$ proposto aos dados experimentais, foram obtidos através da equação (46) aplicada aos dados experimentais. $O$ valor adotado do fator $c$ foi a média aritmética dos seus valores, para a faixa de temperatura analisada, 300 a 573 K. Para uma analise da influència da adoção da média aritmética do fator $c$, como o valor inserido no modelo $C M$ para o calculo de $V_{Z T C}$, foi calculado o seu respectivo desvio padrão. Desta forma o valor de $V_{\text {ZTC }}$ obtido pelo modelo CM é apresentado nas figuras supracitadas, obtido com a média do fator c (linha cheia) e com a variação superior e inferior do desvio padrão (barra de erros). Os valores encontrados estão dentro do intervalo relatado na literatura ${ }^{22}$ e foi observado um bom ajuste dos resultados do modelo CM proposto, apesar das simplificações utilizadas.

Esta apresentação da influência do valor adotado para o fator $c$ no cálculo do valor de $\mathrm{V}_{\mathrm{ZTC}}$ também será mostrada para os resultados obtidos com os dispositivos polarizados na região de saturação, apresentados no item 4.2.2.2 . 


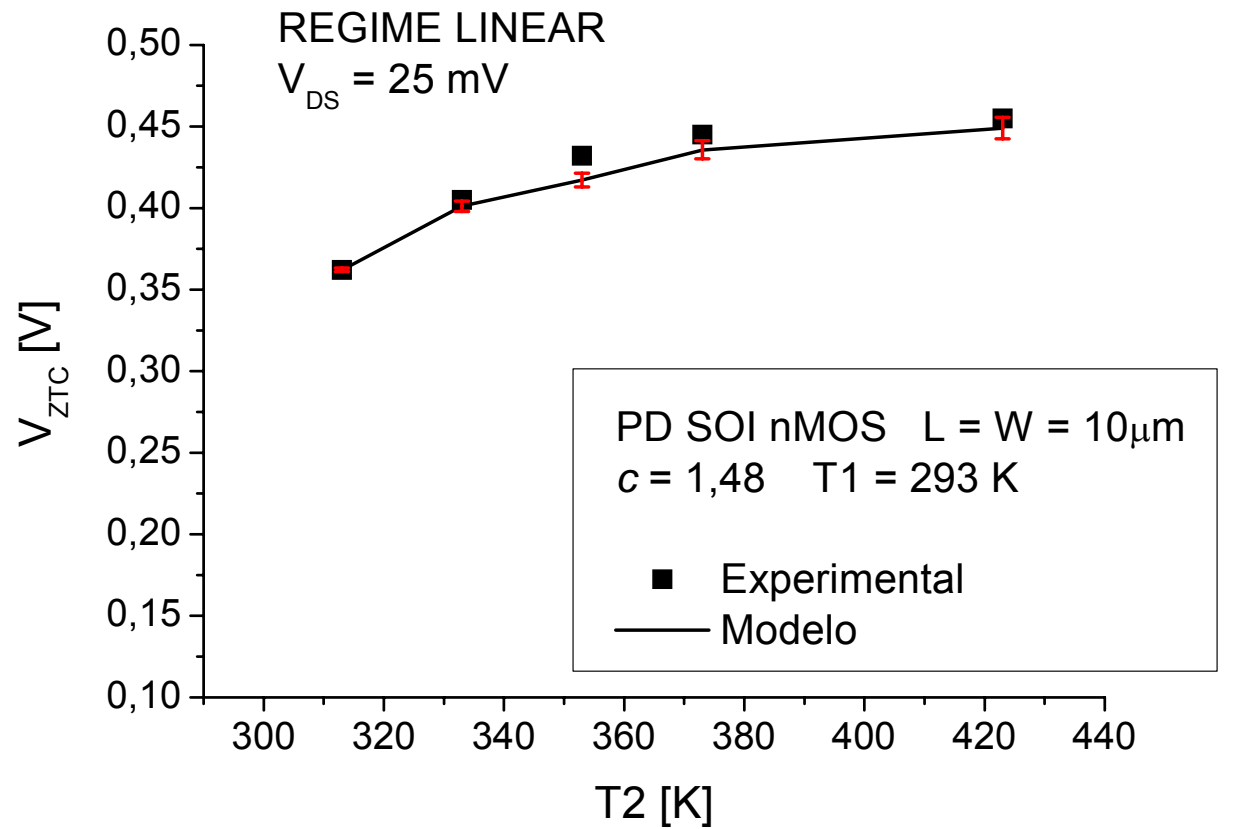

Figura 17: $\mathrm{V}_{\mathrm{ZTC}}$ obtido experimentalmente e através da equação (47) para dispositivos PD SOI nMOS.

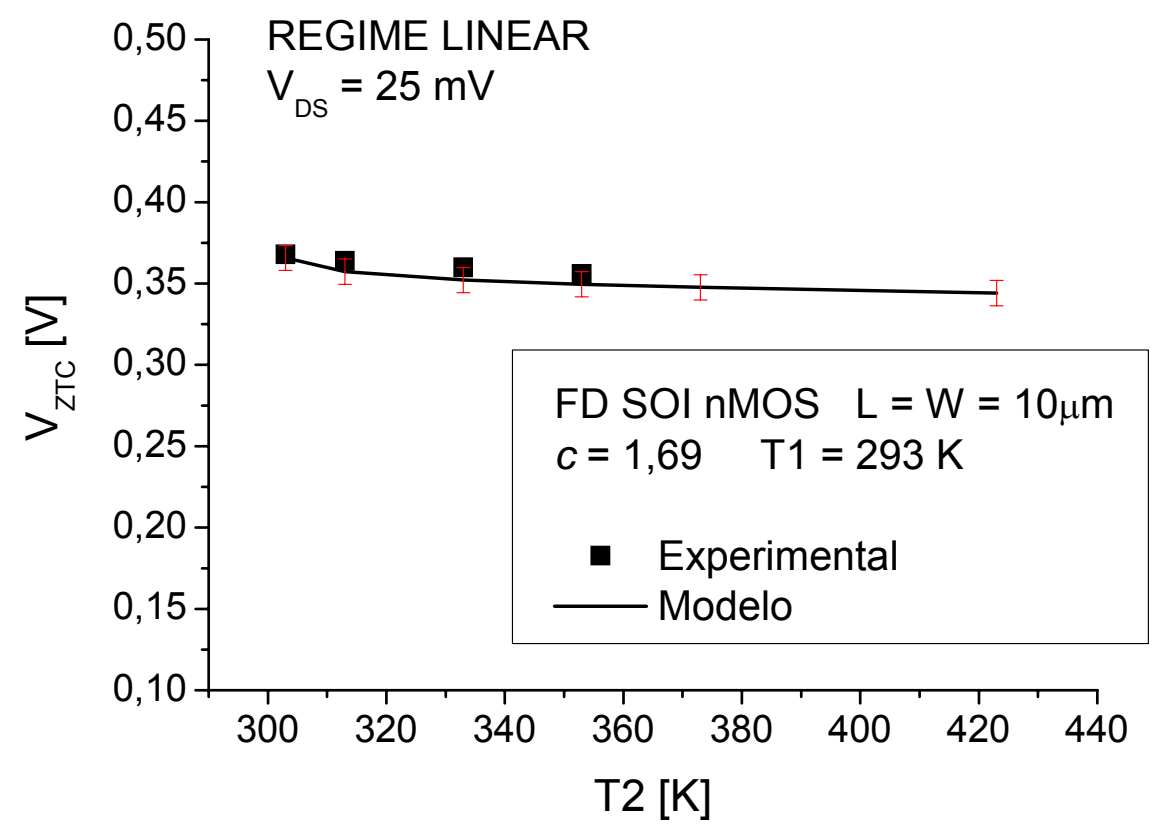

Figura 18: $V_{\text {ZTC }}$ obtido experimentalmente e através da equação (47) para dispositivos FD SOI nMOS. 


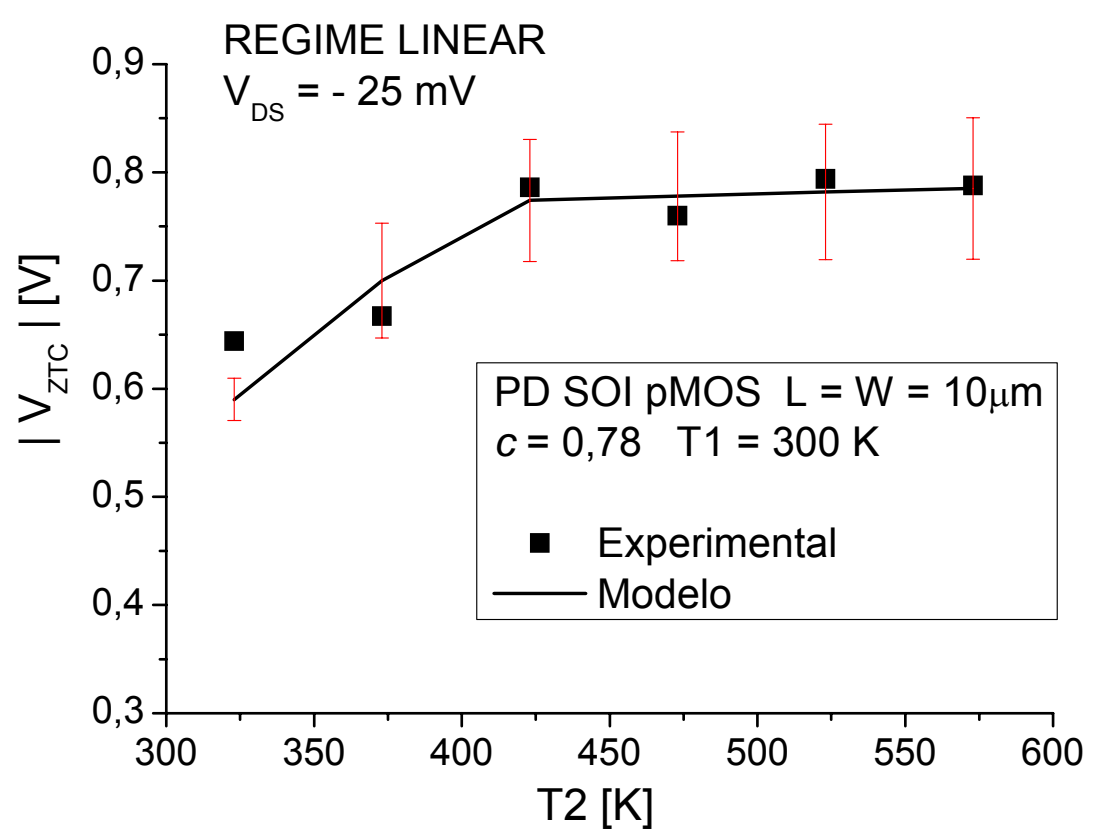

Figura 19: $\mathrm{V}_{\mathrm{ZTC}}$ obtido experimentalmente e através da equação (47) para dispositivos PD SOI pMOS.

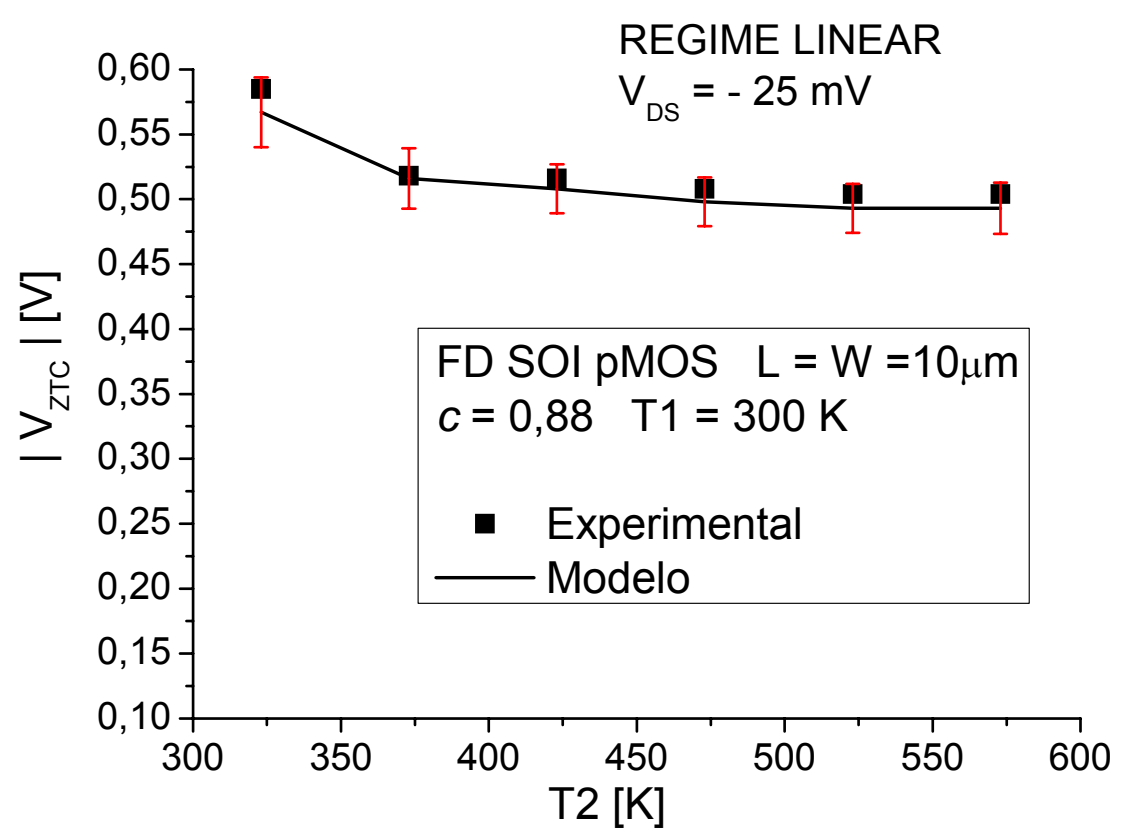

Figura 20: $V_{\text {ZTC }}$ obtido experimentalmente e através da equação (47) para dispositivos FD SOI pMOS. 


\subsubsection{Região de Saturação de Operação}

As curvas $I_{D S} \times V_{G F}$ foram obtidas da mesma forma que os dados extraídos com o transistor polarizado em regime linear, ou seja, através de medidas experimentais com os transistores SOI $n$ e $p$ MOSFETs parcialmente (PD) e totalmente (FD) depletados, e sob diferentes temperaturas, porém, operando na região de saturação de polarização. $\mathrm{Na}$ Figura 21 e na Figura 22 é possível observar a ocorrência do ponto ZTC, obtendo o valor aproximado de $\mathrm{V}_{\mathrm{ZTC}} \simeq 0,32 \mathrm{~V}$ e $\mathrm{V}_{\mathrm{ZTC}} \simeq 0,45 \mathrm{~V}$ para os transistores PD e FD, respectivamente.

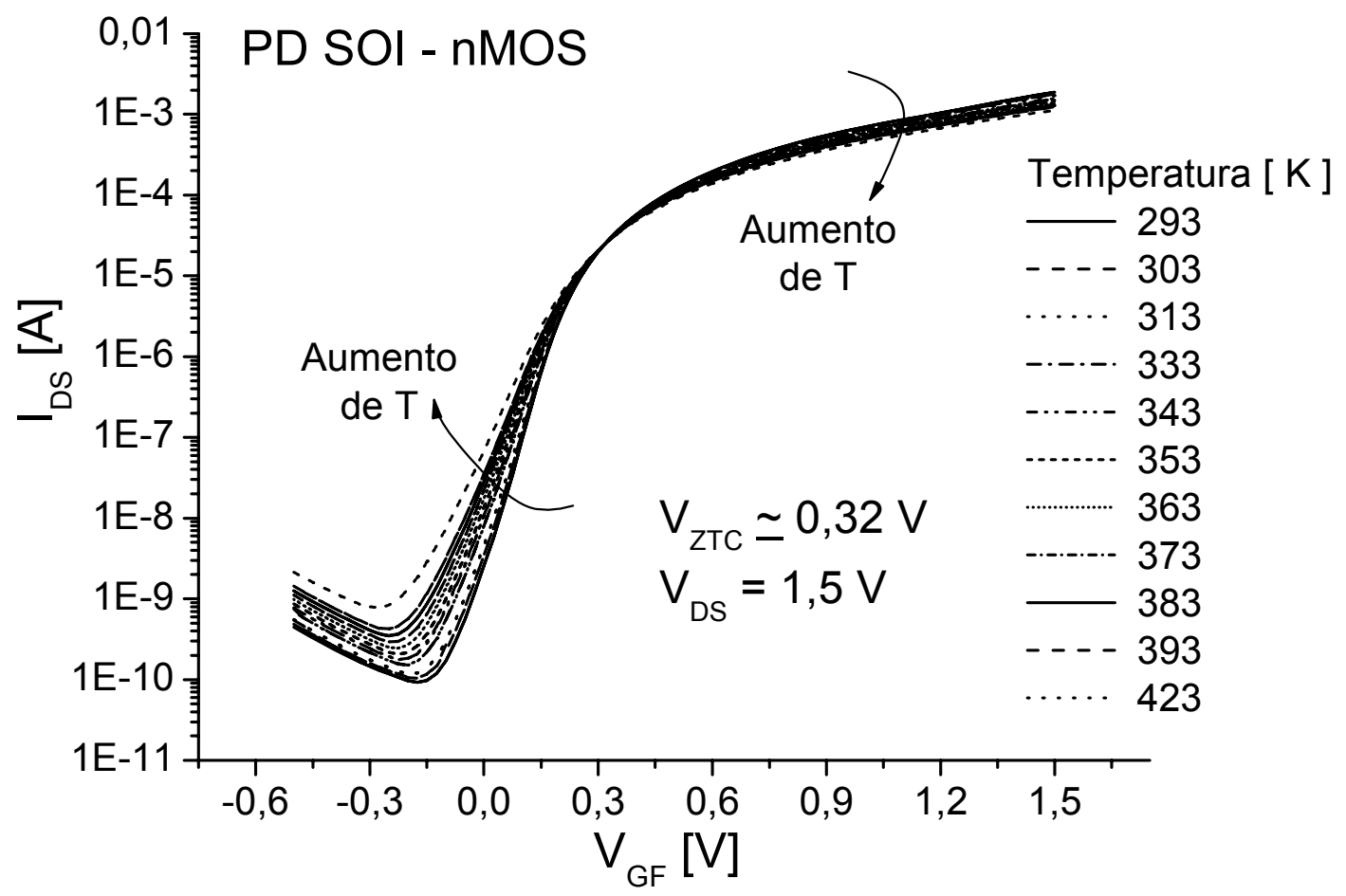

Figura 21: Curvas $I_{D S} \times V_{G F}$ de medidas experimentais do transistor PD-SOI nMOS em diferentes temperaturas. 


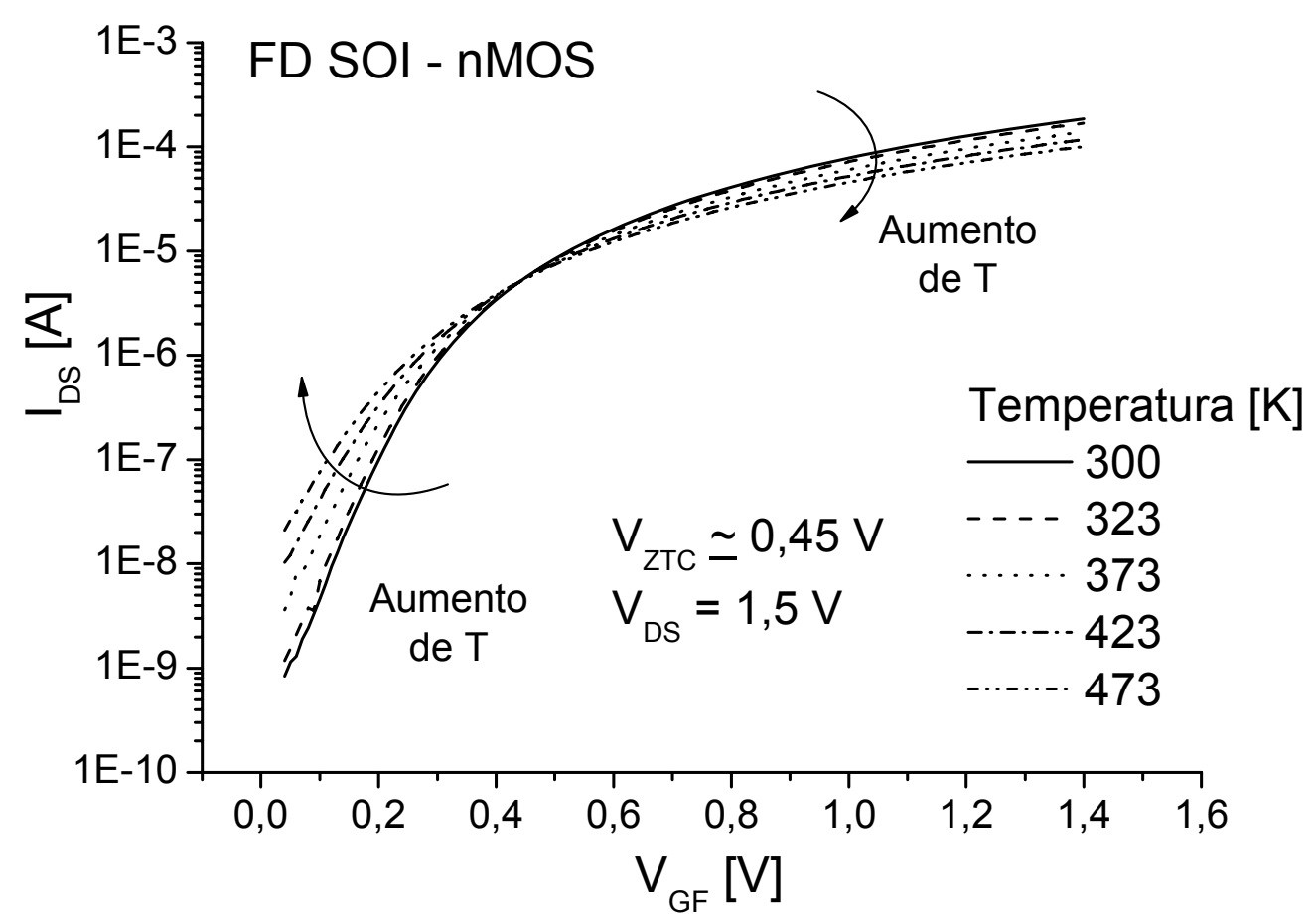

Figura 22: Curvas $I_{D S} \times V_{G F}$ de medidas experimentais do transistor FD-SOI nMOS em diferentes temperaturas.

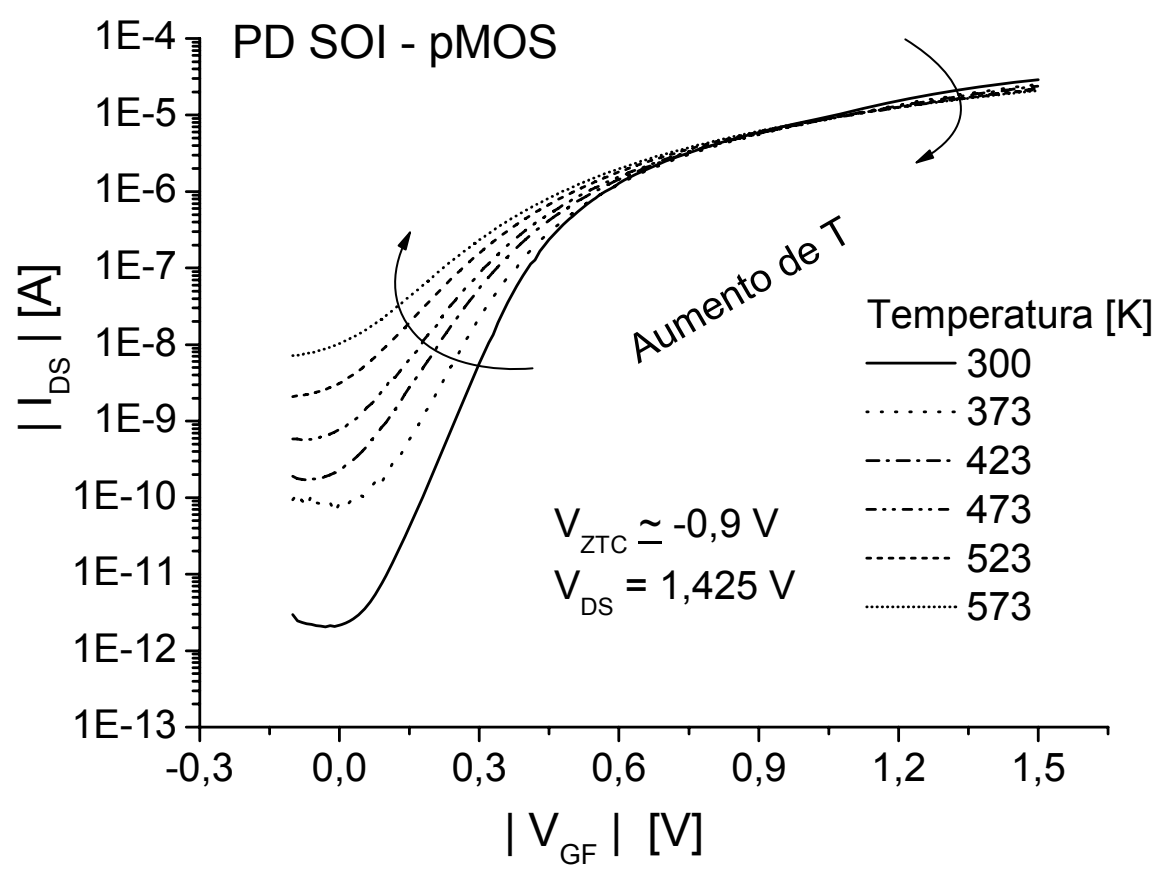

Figura 23: Curvas $I_{D S} \times V_{G F}$ de medidas experimentais do transistor PD-SOI pMOS em diferentes temperaturas. 


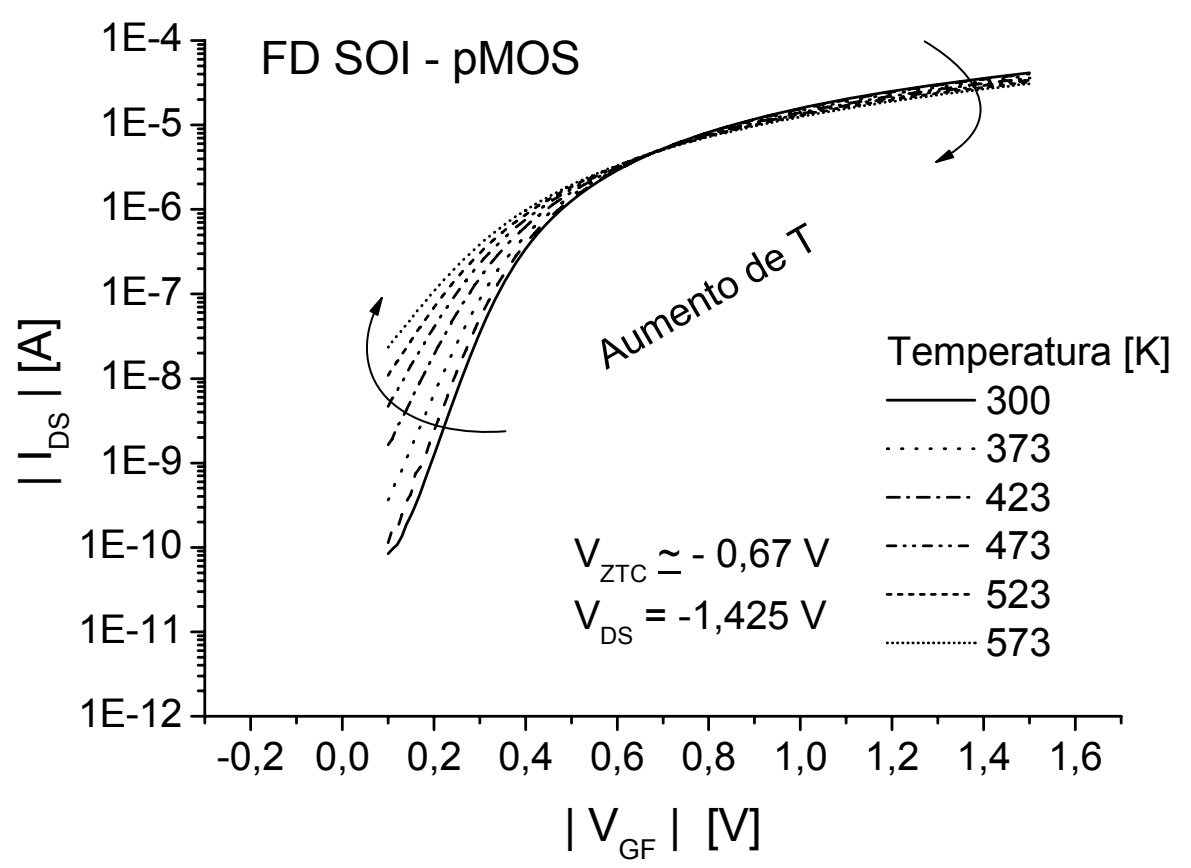

Figura 24: Curvas $I_{D S} \times V_{G F}$ de medidas experimentais do transistor FD-SOI pMOS em diferentes temperaturas.

É possível observar também a ocorrência do ponto ZTC para os dispositivos SOI pMOSFETs na Figura 23 e Figura 24, obtendo o valor aproximado de $\mathrm{V}_{\mathrm{ZTC}} \simeq-0,9 \vee$ e $\mathrm{V}_{\mathrm{ZTC}} \simeq-0,67 \vee$ para os transistores $\mathrm{PD}$ e $\mathrm{FD}$, respectivamente.

Utilizando o modelo simples proposto para operação na região de saturação, através das equações (51), (54) e (55), e os dados experimentais obtidos através das curvas $I_{D S} \times V_{G F}$, foram extraídos os valores de $V_{Z T C}$ em função da temperatura para ambos dispositivos PD e FD SOI $n$ e $p$ MOSFETs, e da Figura 25 a Figura 28 são apresentados os resultados.

Pode ser observado, através das figuras supracitadas, um bom ajuste entre os resultados do modelo $\mathrm{CM}$ proposto e os dados experimentais, apesar das simplificações adotadas. $O$ valor do fator $c$ que garantiu esse bom ajuste, para os dispositivos PD $n$ e $p$ MOSFET foram $c=1.46$ e $c=$ 0.56 , respectivamente. Para os dispositivos FD $n$ e $p$ MOSFETs foram 
encontrados $c=1,63$ e $c=0,81$, respectivamente. Os valores estão dentro da faixa relatada na literatura ${ }^{14}$.

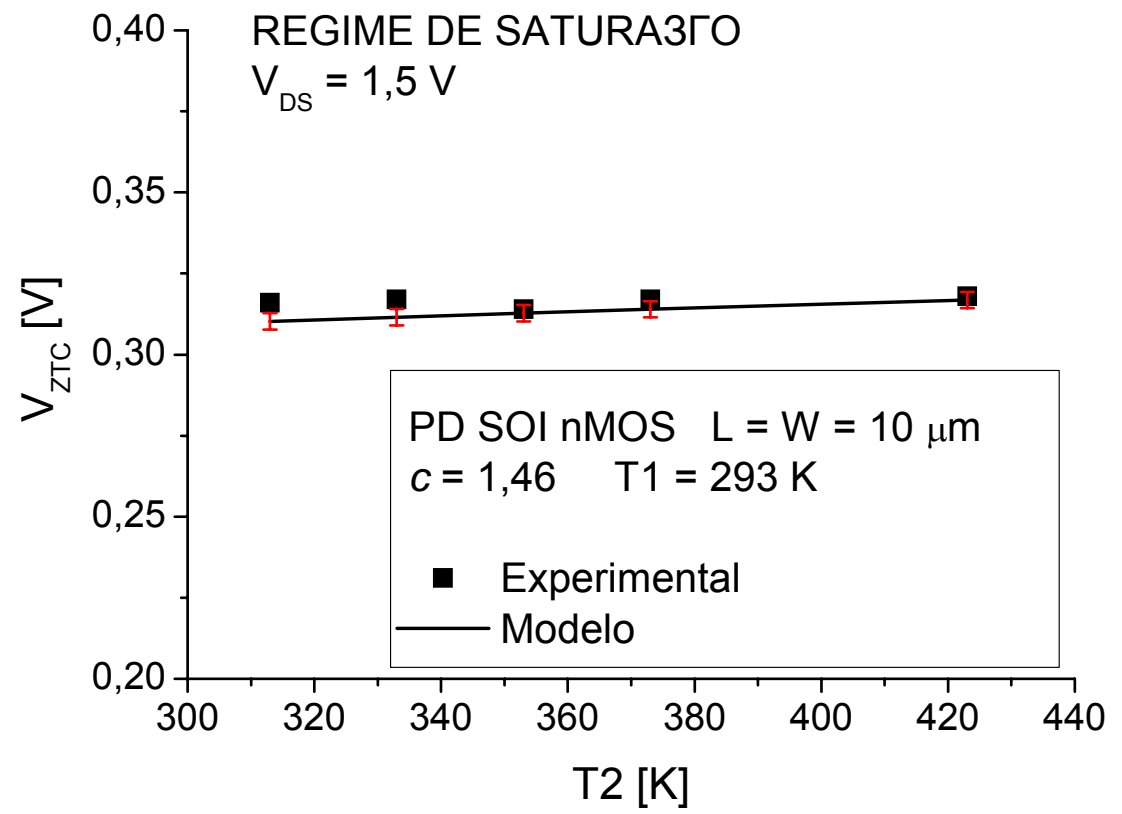

Figura 25: $\mathrm{V}_{\mathrm{ZTC}}$ obtido experimentalmente e pelas equações (51), (54) e (55) para dispositivo PD SOI nMOS.

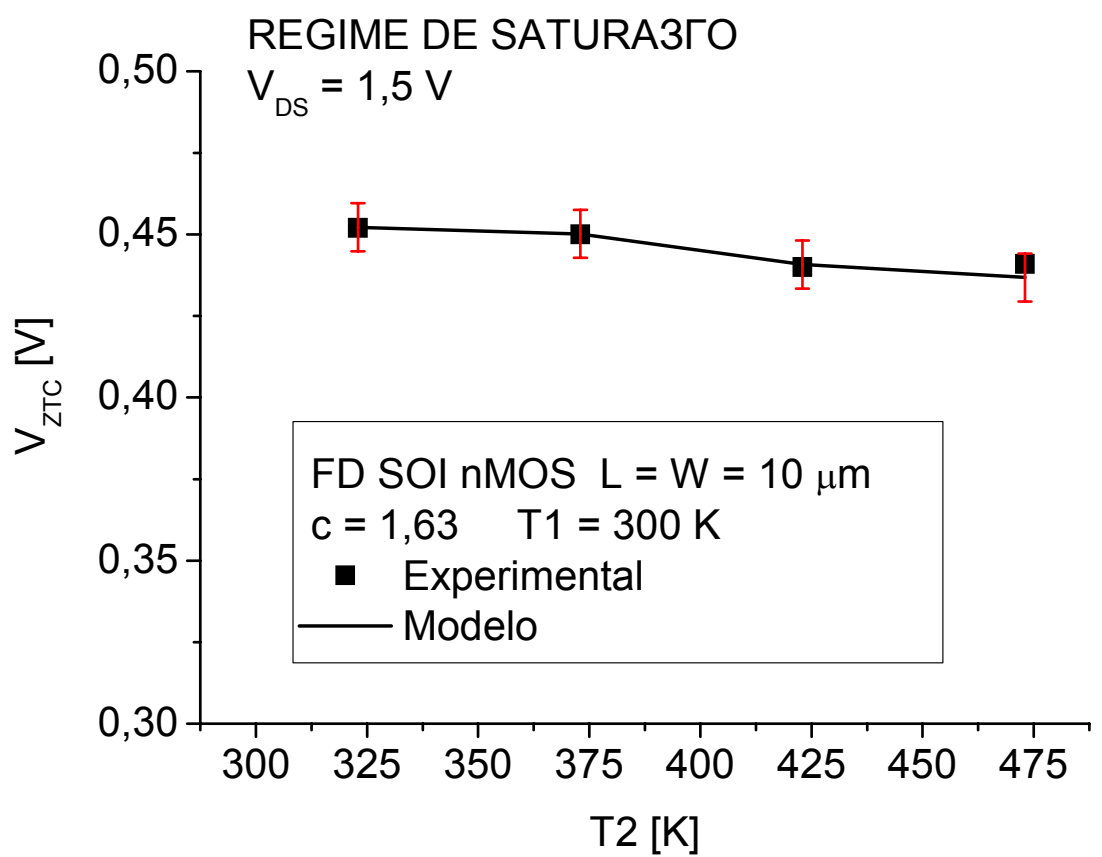

Figura 26: $\mathrm{V}_{\mathrm{ZTC}}$ obtido experimentalmente e pelas equações (51), (54) e (55) para dispositivo FD SOI nMOS. 


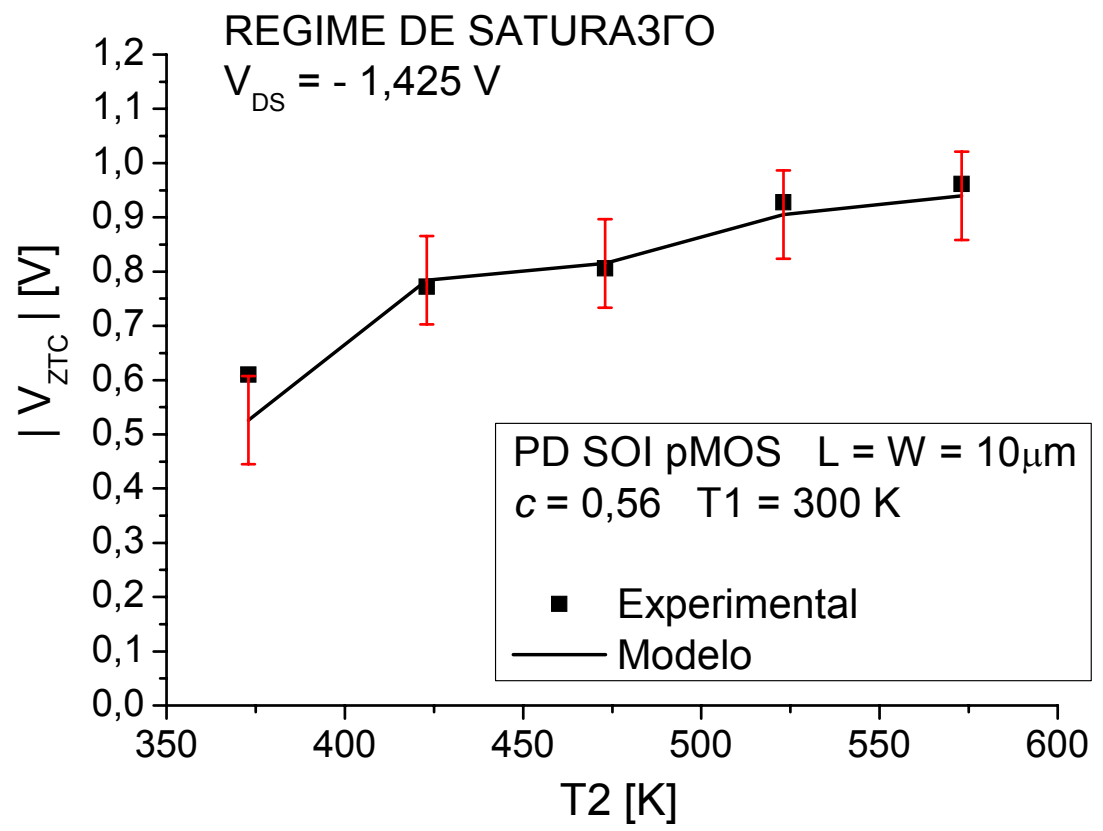

Figura 27: $V_{\mathrm{ZTC}}$ obtido experimentalmente e pelas equações (51), (54) e (55) para dispositivo PD SOI pMOS.

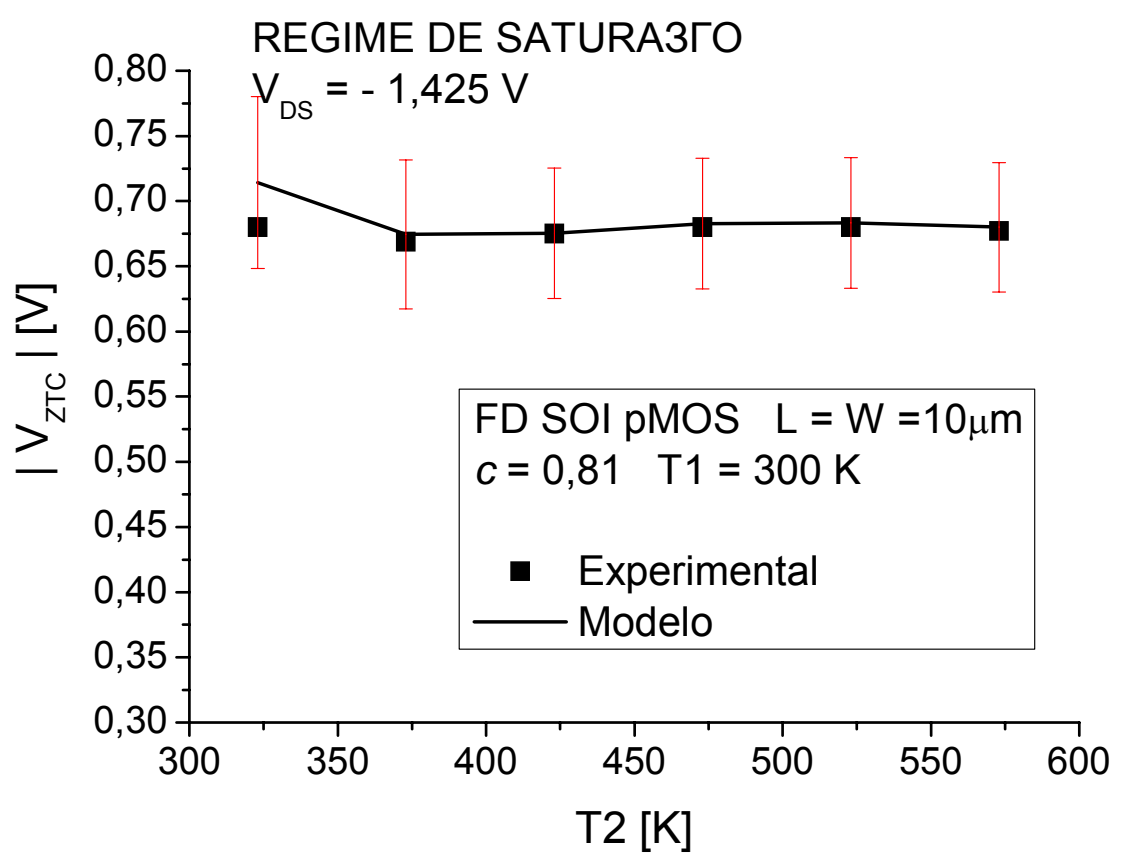

Figura 28: $V_{\mathrm{ZTC}}$ obtido experimentalmente e pelas equações (51), (54) e (55) para dispositivo FD SOI pMOS. 


\section{Estudo da Estabilidade do Ponto Invariante com a Temperatura (ZTC)}

Para os circuitos projetados para aplicações em alta temperatura é desejável que operem no ponto de polarização ZTC, onde a corrente de dreno apresenta zero ou nenhuma variação com a temperatura, e este critério garante que parâmetros como a variação da tensão de offset sejam menos sensíveis a tolerância do balanceamento/casamento dos dispositivos nos circuitos. O crescimento do interesse nos projetos de circuitos que operam de maneira confiável em altas temperaturas aumentou as pesquisas e estudos sobre o ponto de polarização ZTC e sua estabilidade. Neste capítulo é apresentado o estudo da estabilidade do ponto ZTC em função da variação da degradação da mobilidade, comprimento de canal $(L)$ e a tensão de dreno $\left(\mathrm{V}_{\mathrm{DS}}\right)$. Foi realizada também uma avaliação da aplicação do modelo CM proposto ZTC para outra tecnologia diferente da SOI Convencional ou Planar.

\subsection{Influência do fator de degradação da mobilidade com a temperatura ( c ) no ponto ZTC.}

Nesse item é analisada a influência do fator de degradação da mobilidade $(c)$ com a temperatura no ponto invariante com a temperatura $\left(\mathrm{V}_{\mathrm{ZTC}}\right)$, através do modelo $\mathrm{CM}$ proposto no capítulo 4 e dados experimentais, para dispositivos SOI MOSFETs operando em regime totalmente (FD) e parcialmente (PD) depletados. 


\subsubsection{Características físicas dos dispositivos}

Para esta etapa do trabalho, foram utilizados os dispositivos SOI nMOSFETs parcialmente (PD) e totalmente (FD) depletados fabricados com tecnologia SOI CMOS $0.13 \mu \mathrm{m}$. No caso dos dispositivos PD-SOI, a lâmina foi obtida pela técnica UNIBOND com uma espessura de óxido enterrado de $400 \mathrm{~nm}$ e $200 \mathrm{~nm}$ para dispositivos FD-SOI. A estrutura de porta consiste de $2.5 \mathrm{~nm}$ de óxido-nitreto (NO) e um eletrodo com $150 \mathrm{~nm}$ de poli-silício. A técnica STI foi aplicada para o campo de isolação nos dispositivos FD-SOI e PELOX para os transistores PD-SOI. A espessura final da camada de silício é de $30 \mathrm{~nm}$ e 100nm para dispositivos FD e PD, respectivamente.

\subsubsection{Influência do fator de degradação da mobilidade}

Para a análise da influência do fator de degradação da mobilidade com a variação de temperatura (c), equação (46), os seguintes parâmetros foram considerados: a temperatura de referência $\mathrm{T} 1=293 \mathrm{~K}\left(20^{\circ} \mathrm{C}\right)$, a concentração de portadores no filme de silício $N_{\mathrm{af}}=5,5 \times 10^{17} \mathrm{~cm}^{-3}$ para dispositivos SOI nMOSFETs parcialmente depletados (PD-SOI) e $\mathrm{N}_{\mathrm{af}}=$ $1,0 \times 10^{18} \mathrm{~cm}^{-3}$ para dispositivos SOI nMOSFETs totalmente depletados (FDSOI), $t_{o x f}=2,5 \mathrm{~nm}$ para ambos dispositivos e foi observado o valor de $V_{\text {ZTC }}$ em função da variação de temperatura (T2), com os transistores funcionando em regime linear e de saturação através das equações (47) e (50), respectivamente.

\subsubsection{Polarização na Região Linear}

O efeito da variação do parâmetro $c$ é apresentado na Figura 29 e na Figura 30 para dispositivos PD e FD SOI nMOSFET respectivamente, considerando a tensão de dreno $V_{D S}=0,025 \mathrm{~V}$. As figuras mostram que quando ocorre o aumento do fator de degradação da mobilidade com a 
temperatura (c), o valor de $\mathrm{V}_{\mathrm{ZTC}}$ diminui para ambos os dispositivos. Este estudo foi realizado utilizando a expressão (47) e os modelos ${ }^{47}$ que mostram a dependência da tensão de limiar com a variação da temperatura em dispositivos PD e FD SOI MOSFET, através das equações (56) e (57) respectivamente.

$$
\begin{gathered}
\frac{\mathrm{dV}_{\text {thF }}}{\mathrm{dT}}=\frac{\mathrm{d} \phi_{\mathrm{F}}}{\mathrm{dT}}\left[1+\frac{\mathrm{q}}{\mathrm{C}_{\text {oxf }}} \sqrt{\frac{\varepsilon_{\mathrm{Si}} \mathrm{N}_{\mathrm{af}}}{\mathrm{kT} \ln \left(\mathrm{N}_{\mathrm{af}} / \mathrm{ni}\right)}}\right] \quad[\mathrm{V} / \mathrm{K}] \\
\frac{\mathrm{dV}_{\text {thF }}}{\mathrm{dT}}=\frac{\mathrm{d} \phi_{\mathrm{F}}}{\mathrm{dT}} \quad[\mathrm{V} / \mathrm{K}]
\end{gathered}
$$

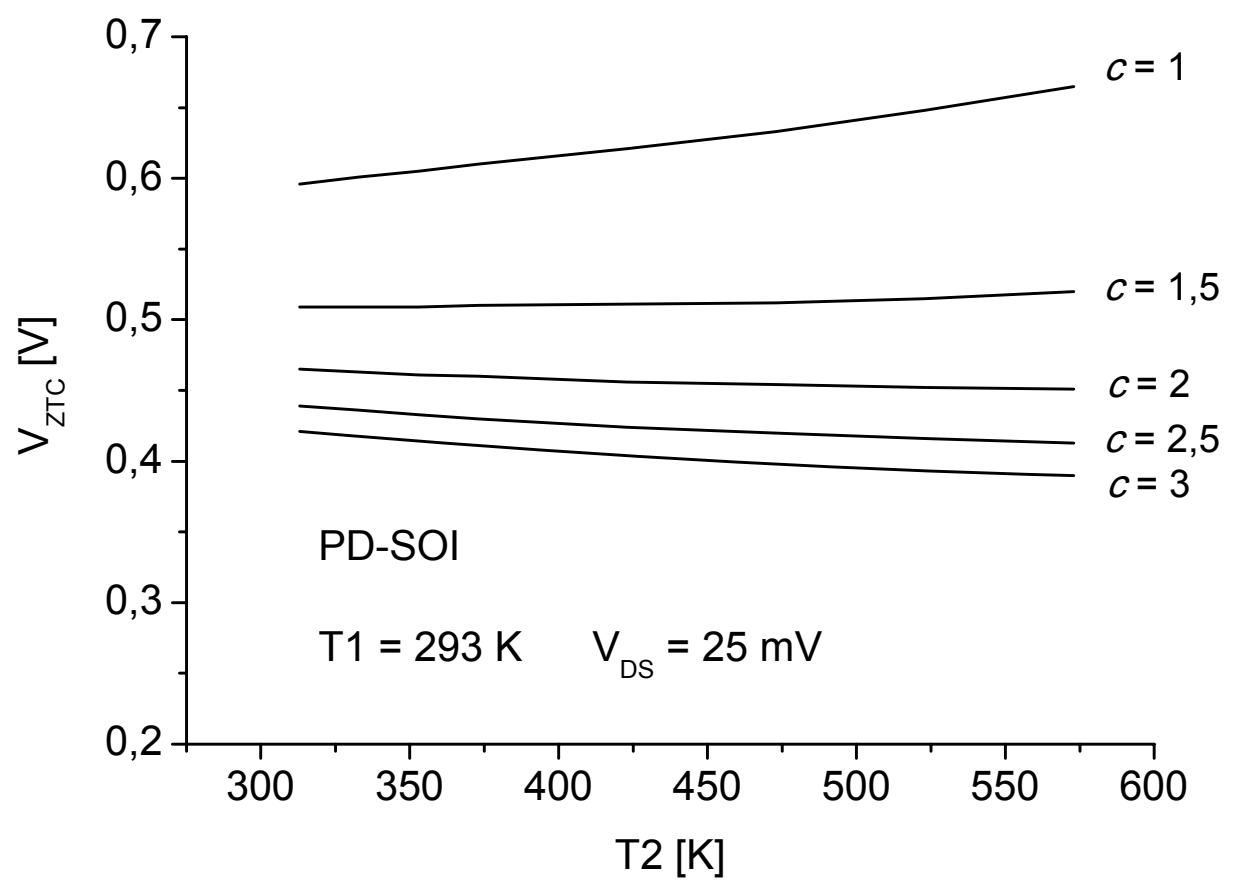

Figura 29: $\mathrm{V}_{\mathrm{ZTC}}$ para dispositivo $\mathrm{PD} \mathrm{SOI}$ - nMOS operando em regime linear em função da temperatura para variações do fator $c$ entre 1 e 3. 


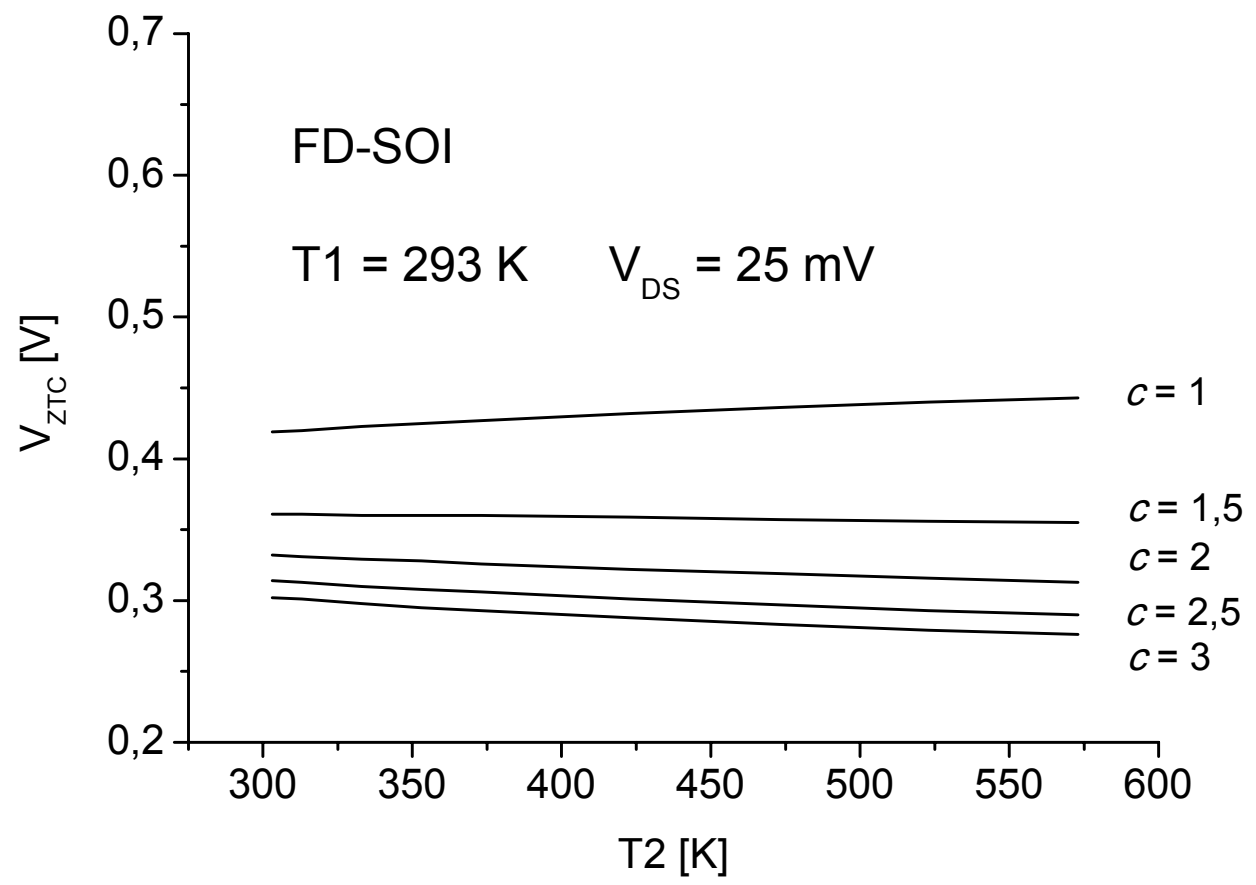

Figura 30: $\mathrm{V}_{\mathrm{ZTC}}$ para dispositivo FD SOI - nMOS operando no regime linear em função da temperatura para variações do fator $c$ entre 1 e 3.

Considerando o dispositivo operando em regime linear, a equação (47) mostra que, com o aumento da temperatura, a variação da tensão de limiar $\left(\mathrm{V}_{\text {th1 }}-\mathrm{V}_{\text {th2 }}\right)$ aumenta e o termo que relaciona a variação de temperatura e o fator $c\left([\mathrm{~T} 1 / \mathrm{T} 2]^{c}\right)$ diminui. A competição destes dois termos depende do fator $c$, que é o responsável pela variação de $V_{\text {ZTC }}$ em função da temperatura. A variação de $\mathrm{V}_{\text {ZTC }}$ em dispositivos PD e FD SOI para temperatura de $313 \mathrm{~K}$ a $573 \mathrm{~K}$, observado na Figura 29 e na Figura 30, é sumarizada na Tabela 2. 
Tabela 2: Variação de $V_{\text {ZTC }}$ para um intervalo de temperatura de $313 \mathrm{~K}$ a $573 \mathrm{~K}$

\begin{tabular}{|c|c|c|}
\hline \multirow{2}{*}{$c$} & \multicolumn{2}{|c|}{$\mathrm{V}_{\mathrm{ZTC}}(313 \mathrm{~K})-\mathrm{V}_{\mathrm{ZTC}}(573 \mathrm{~K})$} \\
\cline { 2 - 3 } & $\mathrm{PD}(\mathrm{mV})$ & $\mathrm{FD}(\mathrm{mV})$ \\
\hline 1 & -69 & -23 \\
\hline 1,5 & -11 & 6 \\
\hline 2 & 14 & 18 \\
\hline 2,5 & 26 & 23 \\
\hline 3 & 32 & 25 \\
\hline
\end{tabular}

$A$ variação de $V_{\text {ZTC }}$ em um intervalo de variação de temperatura de $313 \mathrm{~K}$ a $573 \mathrm{~K}$ nos dispositivos FD é menor do que nos dispositivos PD, uma vez que a variação na tensão de limiar com a temperatura $\mathrm{V}_{\text {thF }}(T)$ depende somente da variação do nível de Fermi. Porém, em dispositivos PD a variação da tensão de limiar com a temperatura $V_{\text {thF }}(T)$ é uma função dependente da redução da profundidade da região de depleção, como mostrado nas equações (56) e (57), respectivamente.

\subsubsection{Polarização na Região de Saturação}

O valor de $V_{\text {ZTC }}(293 \mathrm{~K}$, T2) em função de T2, usando c como parâmetro de variação para dispositivos PD e FD SOI nMOSFETs, considerando a tensão de dreno $\mathrm{V}_{\mathrm{DS}}=1,5 \mathrm{~V}$, é apresentado na Figura $31 \mathrm{e}$ na Figura 32, respectivamente, através das equações (51), (54) e (55). 


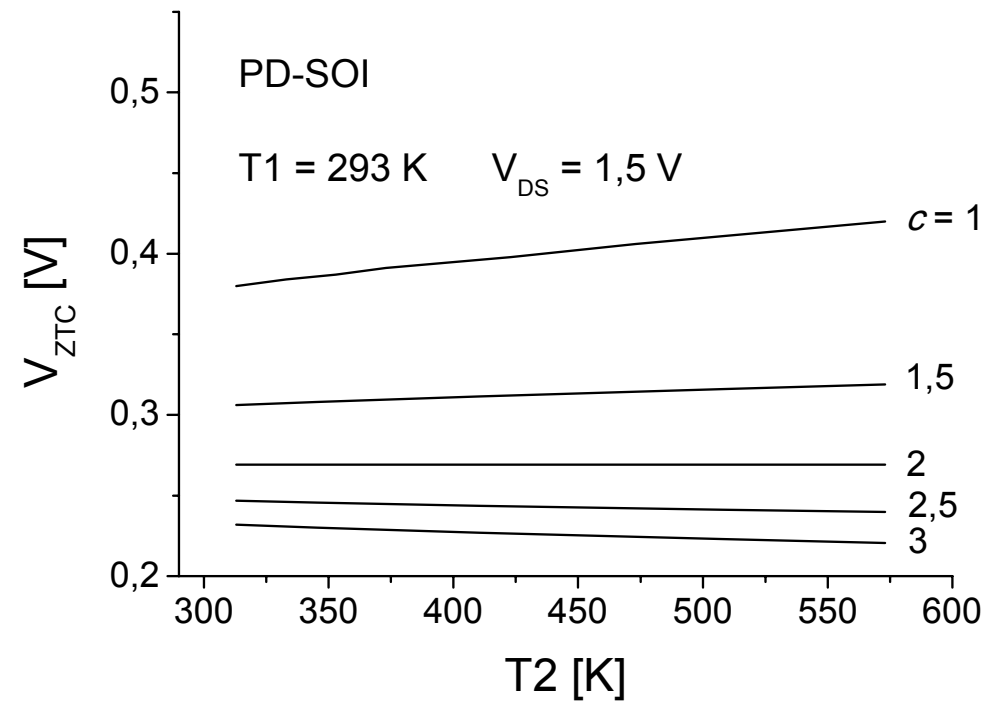

Figura 31: $\mathrm{V}_{\text {ZTC }}$ para dispositivo PD SOI - nMOS operando em regime de saturação em função da temperatura para variação de $c$ entre 1 e 3.

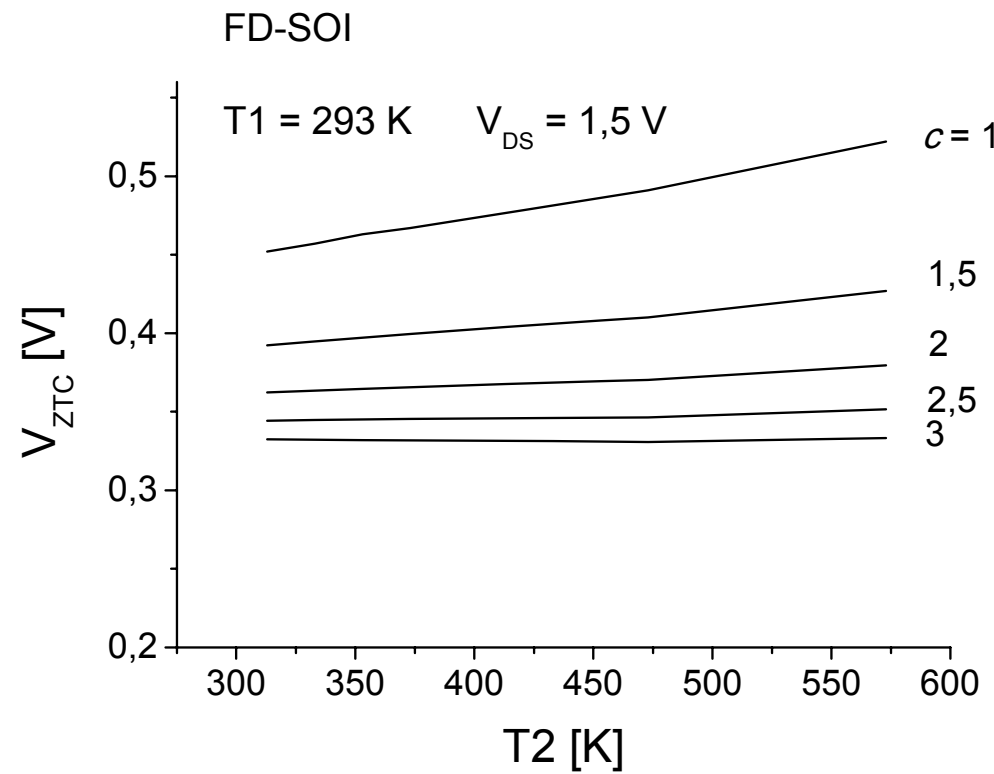

Figura 32: $\mathrm{V}_{\text {ZTC }}$ para dispositivo FD SOI - nMOS operando em regime de saturação em função da temperatura para variação de $c$ entre 1 e 3.

As curvas obtidas, Figura 31 e Figura 32, mostram que, para o aumento do fator de degradação da mobilidade com a temperatura (c) no regime de saturação, o valor de $\mathrm{V}_{\text {ZTC }}$ reduz a uma taxa menor para ambos os dispositivos do que o mesmo observado no regime linear de polarização. 
A relação da tensão de porta no ponto ZTC $\left(\mathrm{V}_{\mathrm{ZTC}}\right)$ pela temperatura T2, em função do parâmetro $c$ para dispositivos PD e FD SOI, é apresentada na Figura 33 e na Figura 34, respectivamente, para os dois regimes de operação linear e saturação. Para os dispositivos PD o valor da variação $\mathrm{dV}_{\mathrm{ZTC}} / \mathrm{dT}$ é praticamente zero no regime linear de operação para $c \simeq 1,7$ e no regime saturação para $c \simeq 2$. Entretanto, para os dispositivos FD isto ocorre para $c \simeq 1,4$ na região linear e $c \simeq 3$ quando os dispositivos estão polarizados na região de saturação.

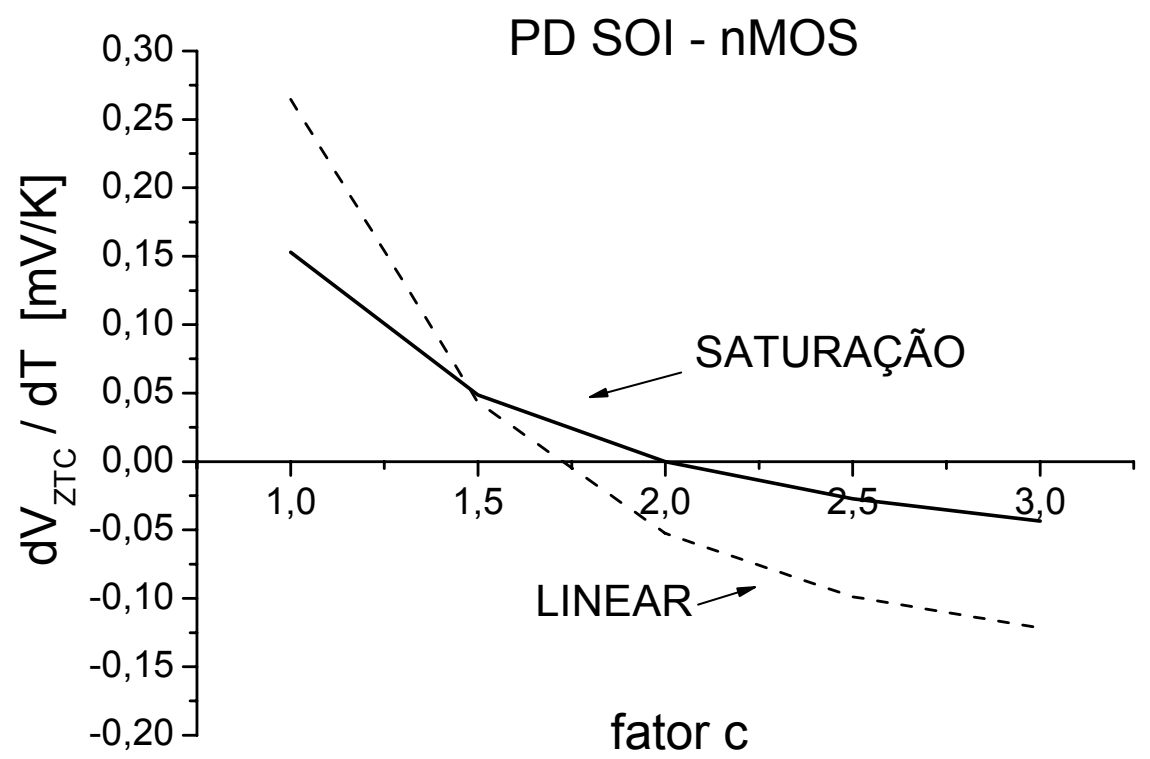

Figura 33: $A$ variação de $V_{Z T C}$ com a temperatura (T2) em função do fator de degradação da mobilidade com a temperatura para dispositivos PD SOI nMOS nas regiões linear e de saturação. 


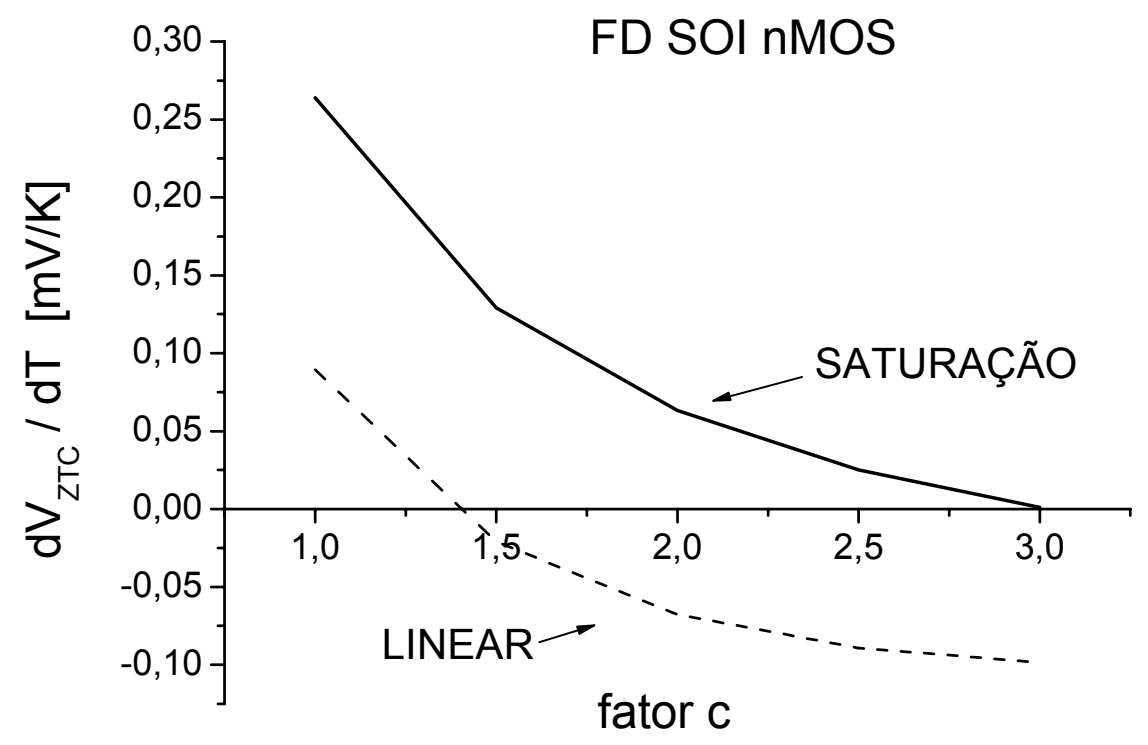

Figura 34: $A$ variação de $V_{\text {ZTC }}$ com a temperatura (T2) em função do fator de degradação da mobilidade com a temperatura para dispositivos FD nas regiões linear e de saturação.

\subsubsection{Sensibilidade do Modelo CM}

O objetivo da análise da sensibilidade do modelo CM proposto, equação (51), foi estudar o impacto que a variação no valor de parâmetros físicos dos dispositivos afeta no valor obtido de $\mathrm{V}_{\text {ZTC. }}$ Os parâmetros como a concentração de dopantes $\left(\mathrm{N}_{\mathrm{af}}\right)$ e a espessura do óxido de porta ( $\left.\mathrm{t}_{\mathrm{oxf}}\right)$, podem sofrer variações ao longo do processo de fabricação, e apresentar diferença do valor de projeto. Desta forma, realizamos a análise do efeito da introdução de uma variação de $10 \%$ no valor dos parâmetros, $N_{\mathrm{af}}=5,5 \mathrm{x}$ $10^{17} \mathrm{~cm}^{-3}$ (PD), $\mathrm{N}_{\mathrm{af}}=1,0 \times 10^{18} \mathrm{~cm}^{-3}$ (FD) e $\mathrm{t}_{\mathrm{oxf}}=2,5 \mathrm{~nm}$, adotados como referência, sobre o valor de $\mathrm{V}_{\mathrm{ZTC}}$ obtido pelo modelo $\mathrm{CM}$ proposto. Para essa análise, a variação $\Delta \mathrm{V}_{\mathrm{ZTC}}$ foi adotada a equação (58). Como sendo:

$$
\Delta \mathrm{V}_{\mathrm{ZTC}}=\mathrm{V}_{\mathrm{ZTC}}\left(\mathrm{N}_{\mathrm{af}}, \mathrm{t}_{\mathrm{oxf}}\right)-\mathrm{V}_{\mathrm{ZTC}} \text { (referência) }
$$


A Figura 35 e a Figura 36 mostram que com o aumento do valor de $\mathrm{N}_{\mathrm{af}}$, o valor de $\Delta \mathrm{V}_{\mathrm{ZTC}}$ também aumenta, já que por sua vez o valor da tensão de limiar aumenta. Por outro lado, quando o valor de $t_{\text {oxf }}$ diminui, o valor de $\Delta \mathrm{V}_{\text {ZTC }}$ diminui, devido ao fato do valor da tensão de limiar também diminuir. Em ambos os casos, a tensão de limiar na temperatura de referência T1 $\left(V_{\text {th1 }}\right)$ é o principal parâmetro das equações (54) e (55).

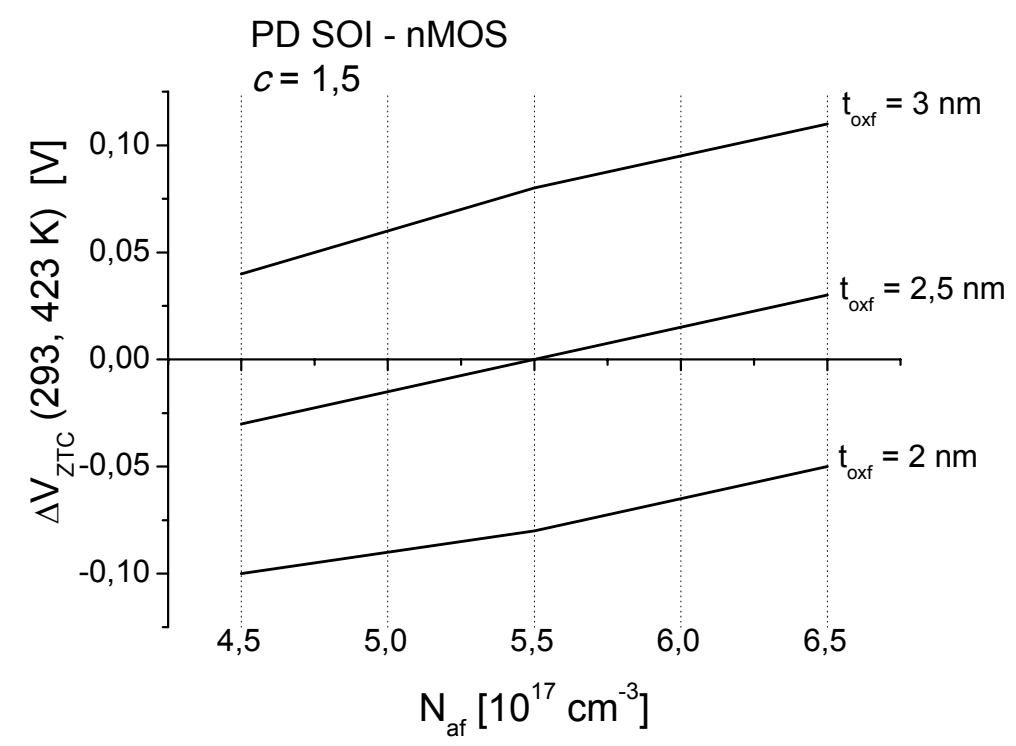

Figura 35: $\Delta \mathrm{V}_{\mathrm{ZTC}}$ para dispositivos PD SOI nMOS

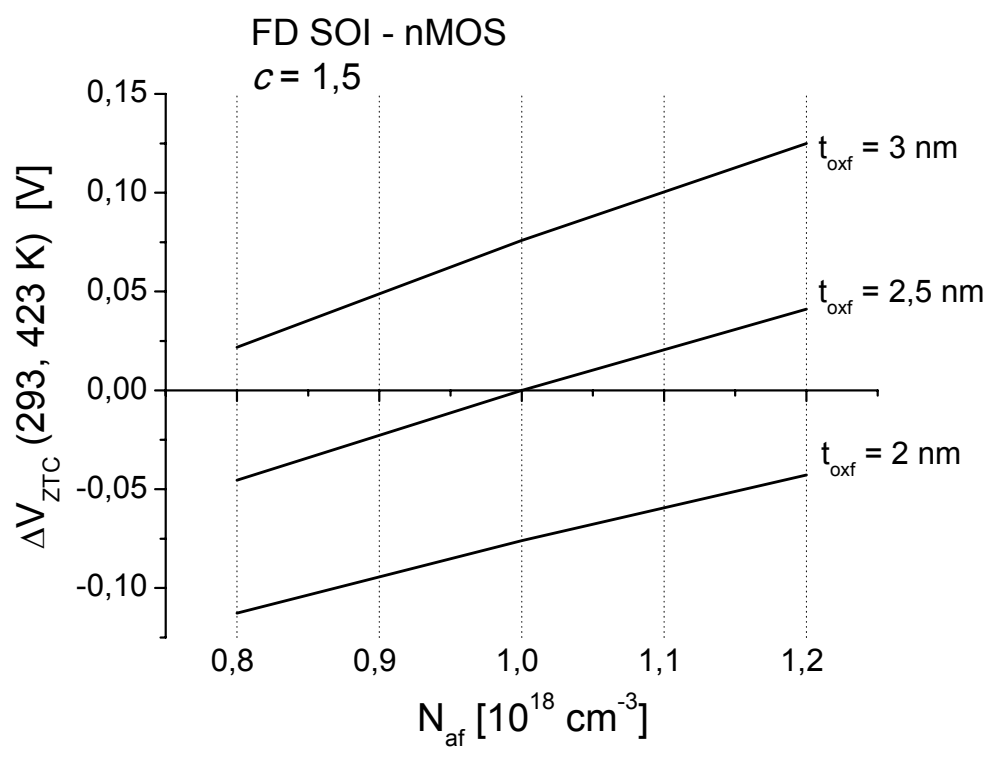

Figura 36: $\Delta \mathrm{V}_{\mathrm{ZTC}}$ para dispositivos FD SOI nMOS 
A Tabela 3 e a Tabela 4 sumarizam os resultados para os dispositivos PD e FD SOI nas regiões linear e de saturação, respectivamente. E como pode ser visto o erro máximo no valor de $\mathrm{V}_{\mathrm{ZTC}}$ é fortemente dependente da variação introduzida nos valores de $\mathrm{N}_{\mathrm{af}}$ e $\mathrm{t}_{\mathrm{oxf}}$. Entretanto a menor variação em $\Delta V_{\text {ZTC }}$ ocorre quando a variação nos valores de $N_{\text {af }}$ e $t_{\text {oxf }}$ é oposta, pelo fato de suas influências serem compensadas no valor de $V_{\text {th1 }}$.

A Tabela 3 mostra que, a influência sobre o valor de $V_{\text {ZTC }}$ observada para os dispositivos PD devido a variação em $\mathrm{N}_{\mathrm{af}}$ e $\mathrm{t}_{\mathrm{oxf}}$. Considerando o valor de referência de $t_{o x f}=2,5 \mathrm{~nm}$ e analisando a variação $\pm 10 \%$ no valor de $N_{a f}$, a influência máxima em $V_{\text {ZTC }}$ é de $3,1 \%$ na região linear e 3,5\% na região de saturação. Ao considerar o valor de referência de $\mathrm{N}_{\mathrm{af}}=5,5 \times 10^{17} \mathrm{~cm}^{-3} \mathrm{e}$ analisando a variação $\pm 10 \%$ no valor de $t_{o x f}$, constatou-se a influência máxima em $V_{\text {ZTC }}$ de 4,6\% na região linear e 7,2\% na região de saturação.

Para os dispositivos FD a mesma análise foi realizada para os parâmetros $\mathrm{t}_{\mathrm{oxf}}$ e $\mathrm{N}_{\mathrm{af}}$ e as suas influências observadas em $\mathrm{V}_{\text {ZTC}}$, apresentada na Tabela 4. A variação máxima observada em $V_{\text {ZTC }}$ é de $11 \%$ operando no regime linear para ambos os parâmetros; e de 5,3\% e 8,4\% no regime de saturação, respectivamente.

Tabela 3: Sensibilidade do método proposto através da análise da variação em $\Delta \mathrm{V}_{\mathrm{ztc}}$ com uma variação de $10 \%$ nos valores de $\mathrm{N}_{\mathrm{af}}$ e $\mathrm{t}_{\text {oxf }}$ para dispositivos PD SOI nMOS operando nos regimes linear e de saturação.

\begin{tabular}{|c|c|c|c|}
\hline \multicolumn{2}{|c|}{ PD SOI } & \multicolumn{2}{|c|}{ Erro em $\Delta \mathrm{V}_{\mathrm{ZTC}}(\%)$} \\
\hline $\mathrm{t}_{\mathrm{oxf}}(\mathrm{nm})$ & $\mathrm{N}_{\mathrm{af}}\left(10^{17} \mathrm{~cm}^{-3}\right)$ & LINEAR & SATURAÇÃO \\
\hline 2,25 & \multirow{3}{*}{4,95} & 7,4 & 10,1 \\
\hline 2,5 & & 3,1 & 3,5 \\
\hline 2,75 & & 12 & 3,2 \\
\hline 2,25 & \multirow{3}{*}{5,5} & 4,6 & 7,2 \\
\hline 2,5 & & Referência & Referência \\
\hline 2,75 & & 4,6 & 6,8 \\
\hline 2,25 & \multirow{3}{*}{6,05} & 18 & 4,4 \\
\hline 2,5 & & 2,9 & 3,0 \\
\hline 2,75 & & 7,7 & 10,2 \\
\hline
\end{tabular}


Tabela 4: Sensibilidade do método proposto através da análise da variação em $\Delta \mathrm{V}_{\mathrm{ztc}}$ com uma variação de $10 \%$ nos valores de $\mathrm{N}_{\mathrm{af}}$ e $\mathrm{t}_{\mathrm{oxf}}$ para dispositivos FD SOI nMOS operando nos regimes linear e de saturação.

\begin{tabular}{|c|c|c|c|}
\hline \multicolumn{2}{|c|}{ FD SOI } & \multicolumn{2}{|c|}{ Erro em $\Delta \mathrm{V}_{\mathrm{ZTC}}(\%)$} \\
\hline $\mathrm{t}_{\mathrm{oxf}}(\mathrm{nm})$ & $\mathrm{N}_{\mathrm{af}}\left(10^{18} \mathrm{~cm}^{-3}\right)$ & LINEAR & SATURAÇÃO \\
\hline 2,25 & \multirow{3}{*}{0,9} & 20 & 13 \\
\hline 2,5 & & 11 & 5,3 \\
\hline 2,75 & & 18 & 2,6 \\
\hline 2,25 & \multirow{3}{*}{1,0} & 10 & 8,4 \\
\hline 2,5 & & Referência & Referência \\
\hline 2,75 & & 11 & 8,4 \\
\hline 2,25 & \multirow{3}{*}{1,1} & 1 & 3,8 \\
\hline 2,5 & & 11 & 5,0 \\
\hline 2,75 & & 20 & 13 \\
\hline
\end{tabular}

\subsection{Influência da dependência da estabilidade do ponto ZTC com o Comprimento de Canal ( $L$ )}

Nesse item é analisada a influência da variação do comprimento de canal $(\mathrm{L})$ no ponto invariante com a temperatura $\left(\mathrm{V}_{\mathrm{ZTC}}\right)$, através de dados experimentais e do modelo CM proposto no capítulo 4, para dispositivos SOI n e pMOSFETs operando em regime parcialmente (PD) depletado.

\subsubsection{Características físicas dos dispositivos}

As estruturas dos transistores usadas nesta etapa do trabalho eram dispositivos SOI MOSFETs parcialmente depletados (PD), modo enriquecimento com canal $\mathrm{n}$ e $\mathrm{p}$. Foram fabricados com tecnologia SOI CMOS $0.13 \mu \mathrm{m}$ no IMEC/Bélgica. Para estes dispositivos a lâmina foi obtida pela técnica UNIBOND com uma espessura de óxido enterrado de $400 \mathrm{~nm}$. 
A estrutura de porta consiste de $2,5 \mathrm{~nm}$ de óxido-nitreto (NO) e um eletrodo com $150 \mathrm{~nm}$ de poli-silício ( $\mathrm{N}+$ para nMOSFET e $\mathrm{P}+$ para pMOSFET). A técnica PELOX foi aplicada para o campo de isolação e a espessura final da camada de silício é de $100 \mathrm{~nm}$.

Após a definição da porta, uma implantação iônica de baixa energia de arsênio foi realizada para a formação das extensões rasas de Fonte e Dreno, seguida de uma implantação de boro em ângulo para formação da estrutura halo para controlar os efeitos de canal curto nos nMOSFETs. O comprimento da estrutura halo está em torno de $65 \mathrm{~nm}$.

No caso dos pMOSFETs foi realizada a implantação de $\mathrm{BF}_{2}$ para a formação das extensões rasas das regiões de Fonte e Dreno e arsênio na implantação iônica para formação da estrutura halo.

\subsubsection{Resultados e Discussão}

A tensão de limiar, na região linear de operação dos transistores, foi extraída pelo método da extrapolação linear, em que extrapola-se a região linear (região de triodo) da curva $\mathrm{I}_{\mathrm{DS}} \times \mathrm{V}_{\mathrm{GF}}$, obtida com $\mathrm{V}_{\mathrm{DS}}$ constante, até o cruzamento com o eixo de $\mathrm{V}_{\mathrm{GF}}$, no ponto de máxima transcondutância $\left(\mathrm{g}_{\mathrm{m}}\right)^{39}$. Com os dispositivos operando na região de saturação, a tensão de limiar foi extraída pelo método $\mathrm{RM}^{56}$ (ratio method), que determina a tensão de limiar através da relação de $I_{D S}$ pela raiz quadrada da transcondutância $\left(\mathrm{g}_{\mathrm{m}}\right)$ versus a tensão de porta, ou seja, extrapolando-se a região linear da curva obtida por $I_{D S} /\left(g_{m}\right)^{1 / 2}$ versus $V_{G F}$ até o cruzamento no eixo de $V_{G F}$.

Para a verificação da influência do comprimento de canal (L) dos transistores na determinação da tensão de polarização no ponto invariante com a temperatura $\left(\mathrm{V}_{\mathrm{ZTC}}\right)$ foram avaliados os resultados experimentais de dispositivos parcialmente depletados (PD SOI) dos tipos n e p-MOSFETs com diferentes geometrias. Os dispositivos testados foram medidos 
operando nas regiões de operação linear $\left(V_{D S}=25 \mathrm{mV}\right)$ e de saturação $\left(\mathrm{V}_{\mathrm{DS}}=1,425 \mathrm{~V}\right)$ para temperaturas entre $300 \mathrm{~K}$ e $573 \mathrm{~K}$.

Da Figura 37 a Figura 40 é mostrada a tensão de polarização no ponto ZTC ( $\mathrm{V}_{\text {ZTC }}$ ) obtida dos dados experimentais e através do modelo CM proposto $^{57}$ para dispositivos PD SOI nMOS e pMOS, operando nos regimes linear e de saturação, para dispositivos com comprimentos de canal ( $L$ ) de 0,5, 1,5 e $10 \mu \mathrm{m}$. O valor de $\mathrm{V}_{\mathrm{ZTC}}$ foi calculado pelo modelo CM proposto usando as equações apresentadas (47) para avaliação no regime linear e (51, 54 e 55) para operação em regime de saturação, e o valor utilizado do parâmetro de degradação da mobilidade com a temperatura ( c ) foi o valor da média aritmética do mesmo para a faixa de temperatura considerada.

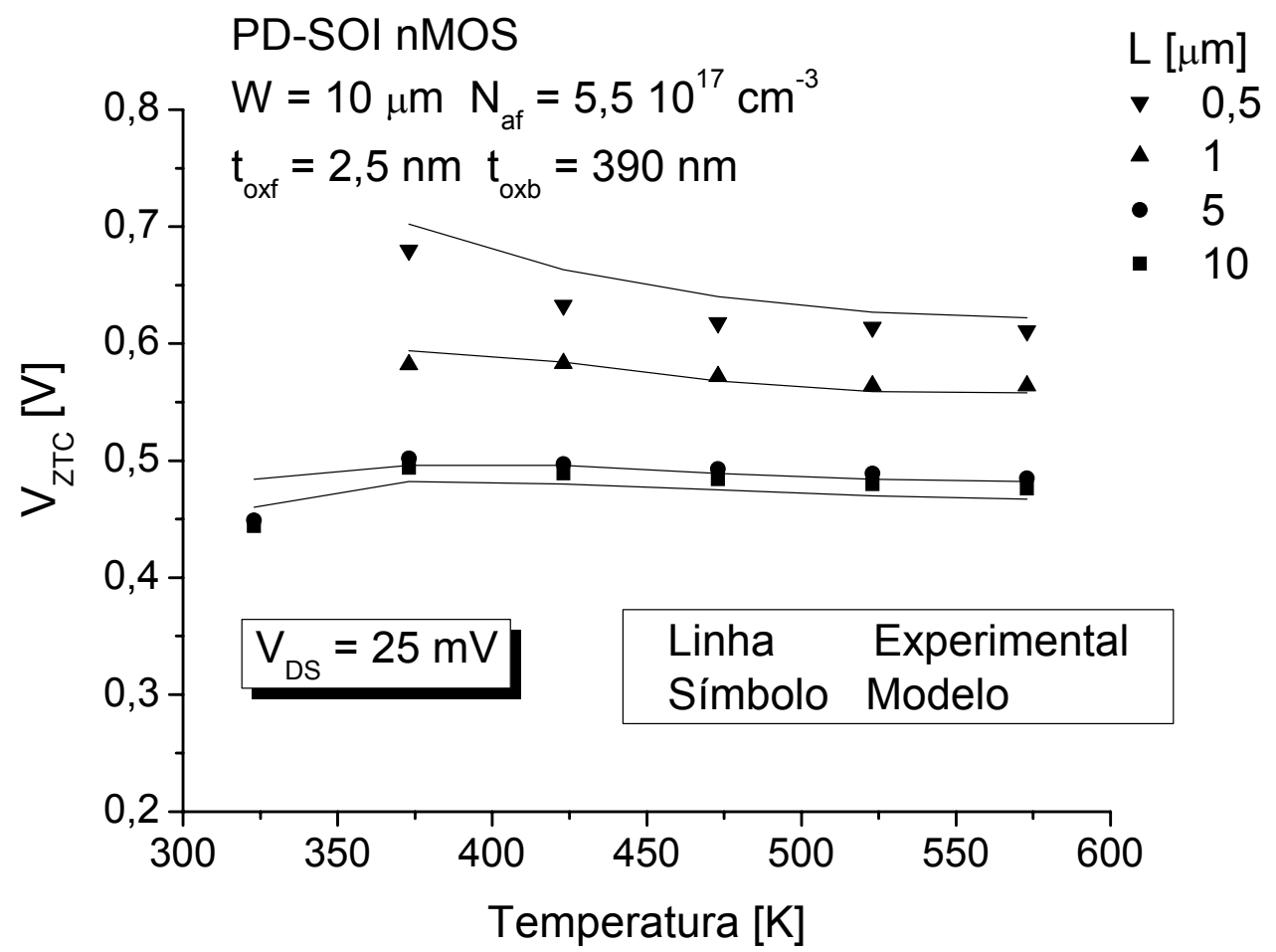

Figura 37: Tensão de polarização no ponto ZTC $\left(\mathrm{V}_{\mathrm{ZTC}}\right)$ obtido experimentalmente e pelo modelo simples proposto para dispositivos PD nMOS SOI operando na região linear, para valores de comprimentos de canal (L) de $0,5,1,5$ and $10 \mu \mathrm{m}$. 


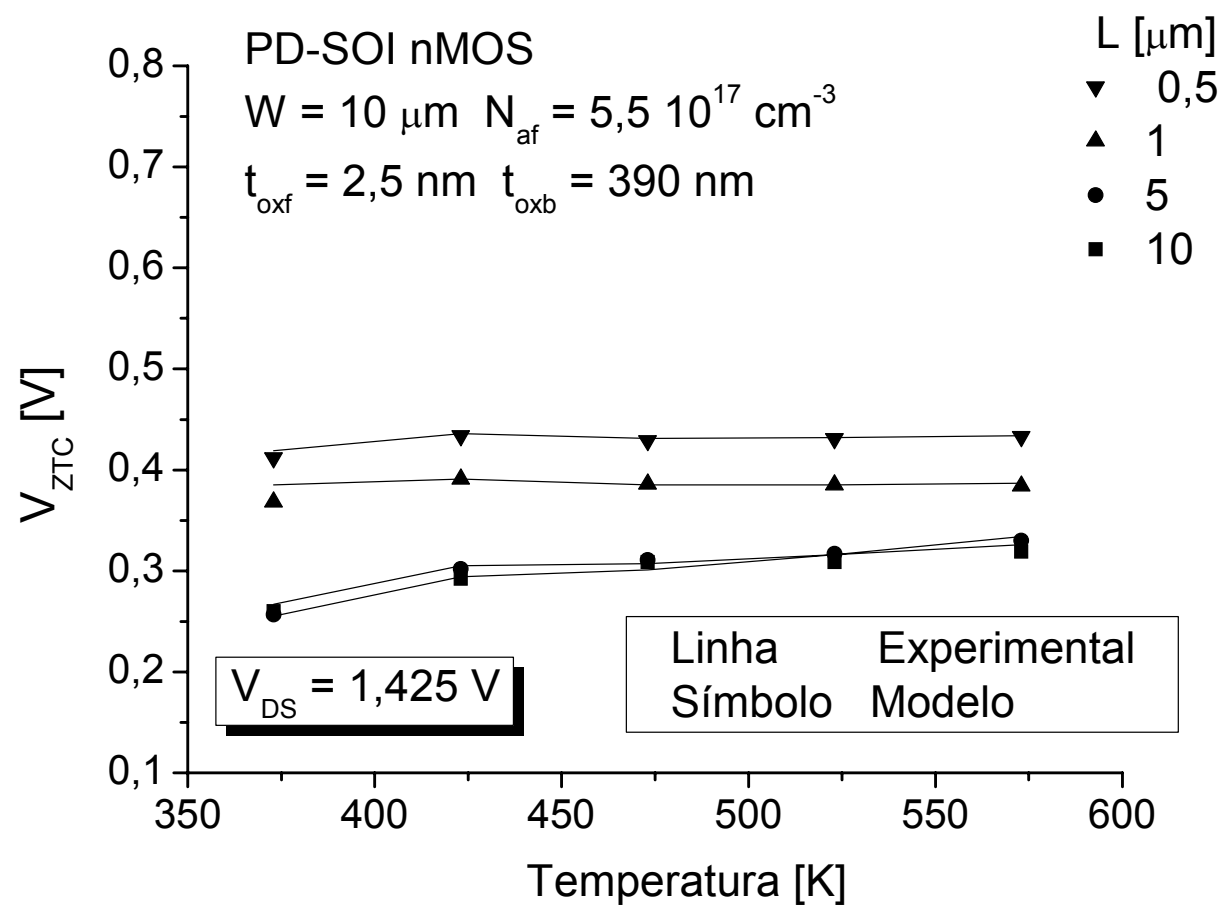

Figura 38: Tensão de polarização no ponto ZTC $\left(\mathrm{V}_{\mathrm{ZTC}}\right)$ obtido experimentalmente e pelo modelo simples proposto para dispositivos PD nMOS SOI operando na região de saturação, para valores de comprimentos de canal (L) de $0,5,1,5$ e $10 \mu \mathrm{m}$.

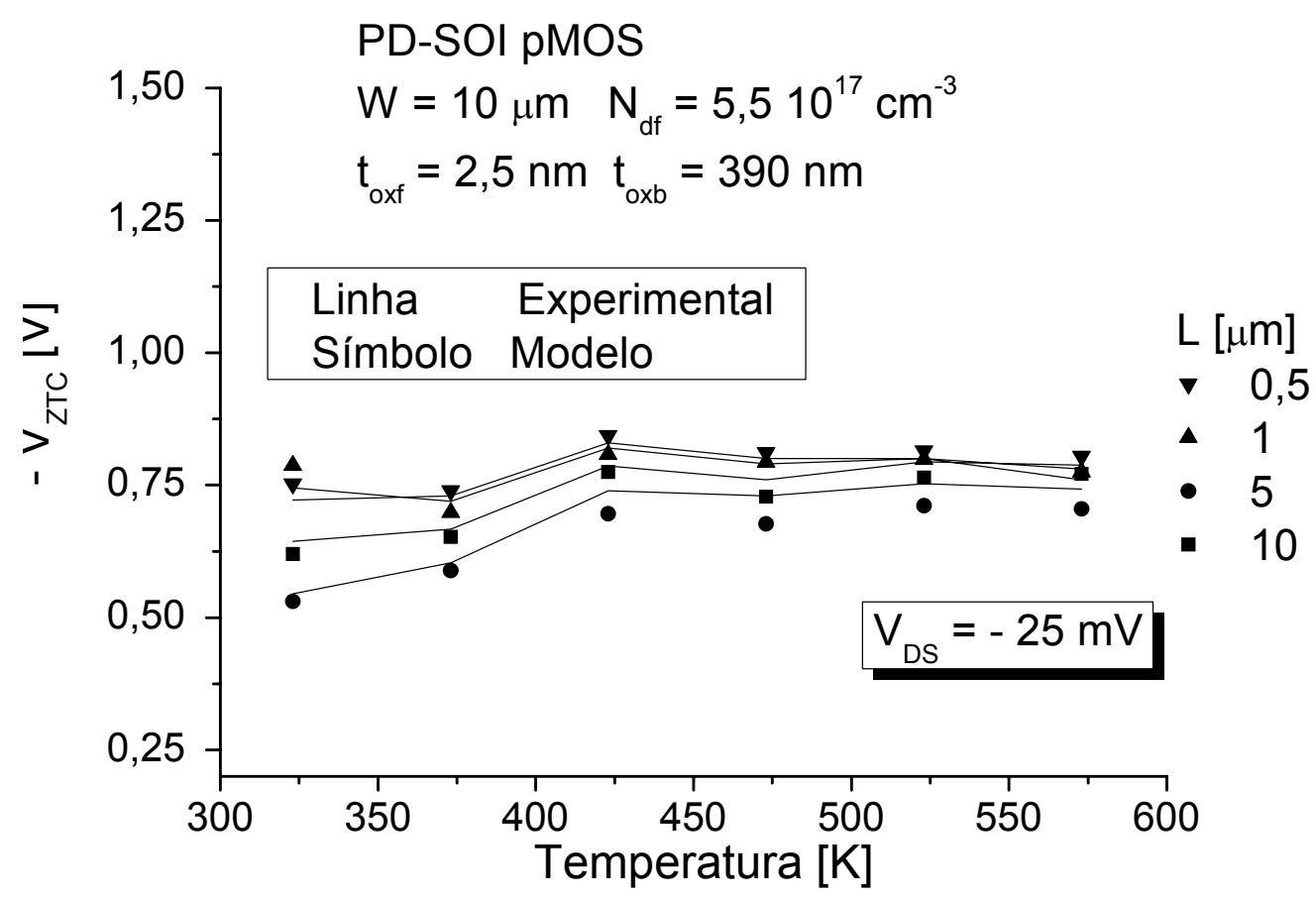

Figura 39: Tensão de polarização no ponto ZTC $\left(\mathrm{V}_{\mathrm{ZTC}}\right)$ obtido experimentalmente e pelo modelo simples proposto para dispositivos PD pMOS SOI operando nas região linear, para valores de comprimentos de canal (L) de 0,5,1,5 e $10 \mu \mathrm{m}$. 


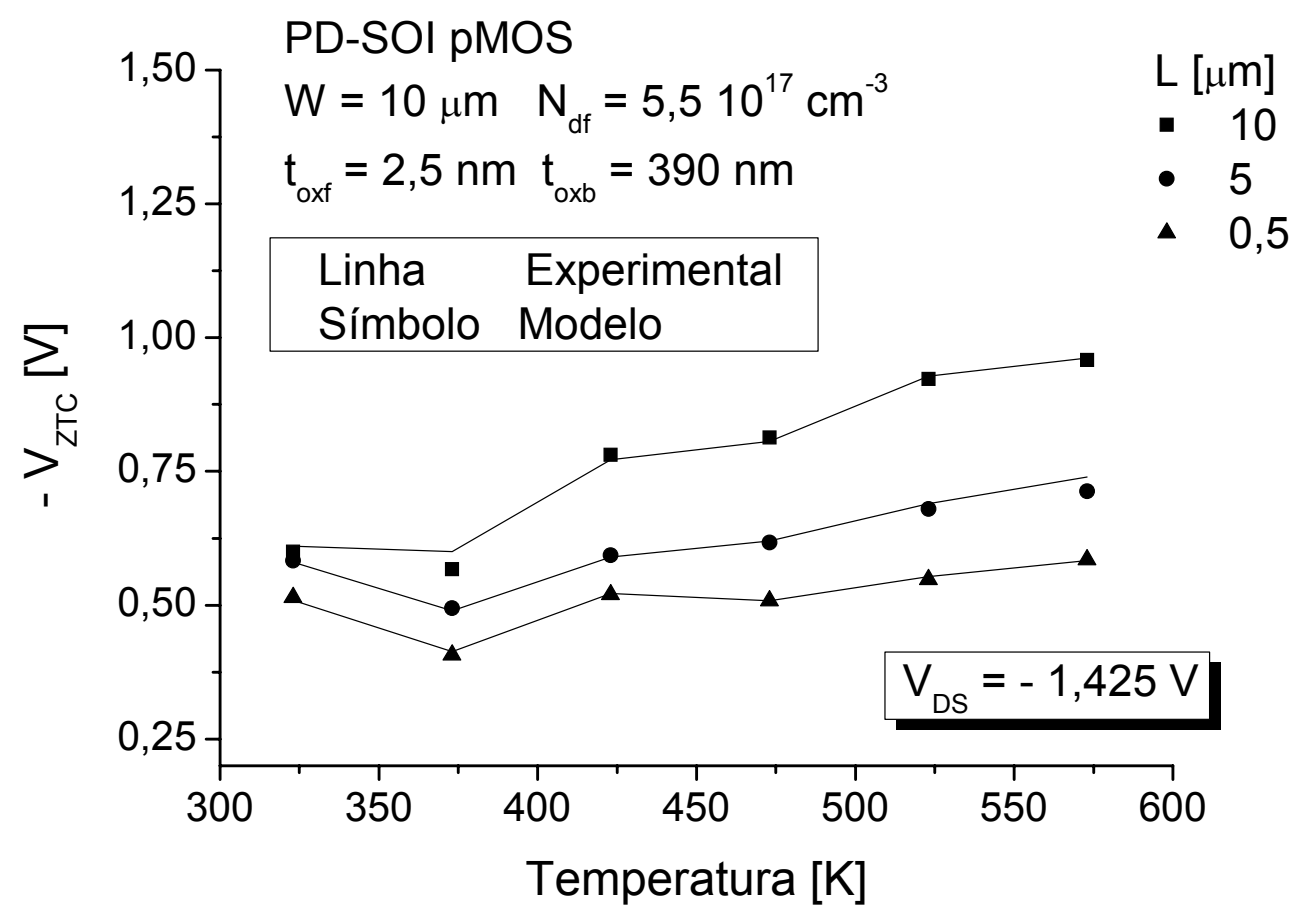

Figura 40: Tensão de polarização no ponto ZTC $\left(V_{\text {ZTC }}\right)$ obtido experimentalmente e pelo modelo simples proposto para dispositivos PD pMOS SOI operando na região de saturação, para valores de comprimentos de canal $(L)$ de $0,5,5$ e $10 \mu \mathrm{m}$.

Os dados apresentados na Figura 37 e na Figura 38 mostram que para os dispositivos PD SOI nMOS, operando na região de saturação, o valor de $\mathrm{V}_{\mathrm{ZTC}}$ é menor do que para os mesmos dispositivos em regime linear, entretanto os dispositivos PD SOI pMOS mostram que $\mathrm{V}_{\mathrm{ZTC}}$ tem os mesmo nível para ambas as regiões.

A tecnologia SOI MOSFET atual produz dispositivos com a estrutura halo $58,59,60$, usadas para minimizar os problemas de escalamento e controlar os efeitos de canal curto. Estes processos de implantação resultam em perfis de dopagem não uniformes ao longo do comprimento do canal do dispositivo, provocando o aumento do efeito reverso de canal curto (RSCE reverse short-channel effect), e este efeito pode ser observado no comportamento da tensão de limiar. Como conseqüência, o mesmo efeito ocorre na tensão de polarização no ponto ZTC $\left(\mathrm{V}_{\mathrm{ZTC}}\right)$, devido a sua dependência direta com a tensão de limiar, e pode ser visto através da variação de $V_{\text {ZTC }}$ em função do comprimento de canal (L), mostrado na 
Figura 41 para dispositivos PD SOI nMOS nas regiões linear e de saturação de operação. As estruturas pMOS não possuem a região de halo, portanto, o efeito citado não foi observado. Para os dispositivos pMOS, Figura 42 para região linear e Figura 43 em operação no regime de saturação, foi observado o comportamento de redução da tensão $\mathrm{V}_{\mathrm{ZTC}}$ para menores comprimentos de canal $(L)$, indicando a ocorrência do efeito de canal curto sobre a tensão de limiar $\left(\mathrm{V}_{\mathrm{thF}}\right)$, que atua diretamente sobre $\mathrm{V}_{\mathrm{ZTC}}$, ou seja, a tensão de limiar aumenta com a diminuição do comprimento de canal (L), e $\mathrm{V}_{\mathrm{ZTC}}$ apresenta o mesma tendência.

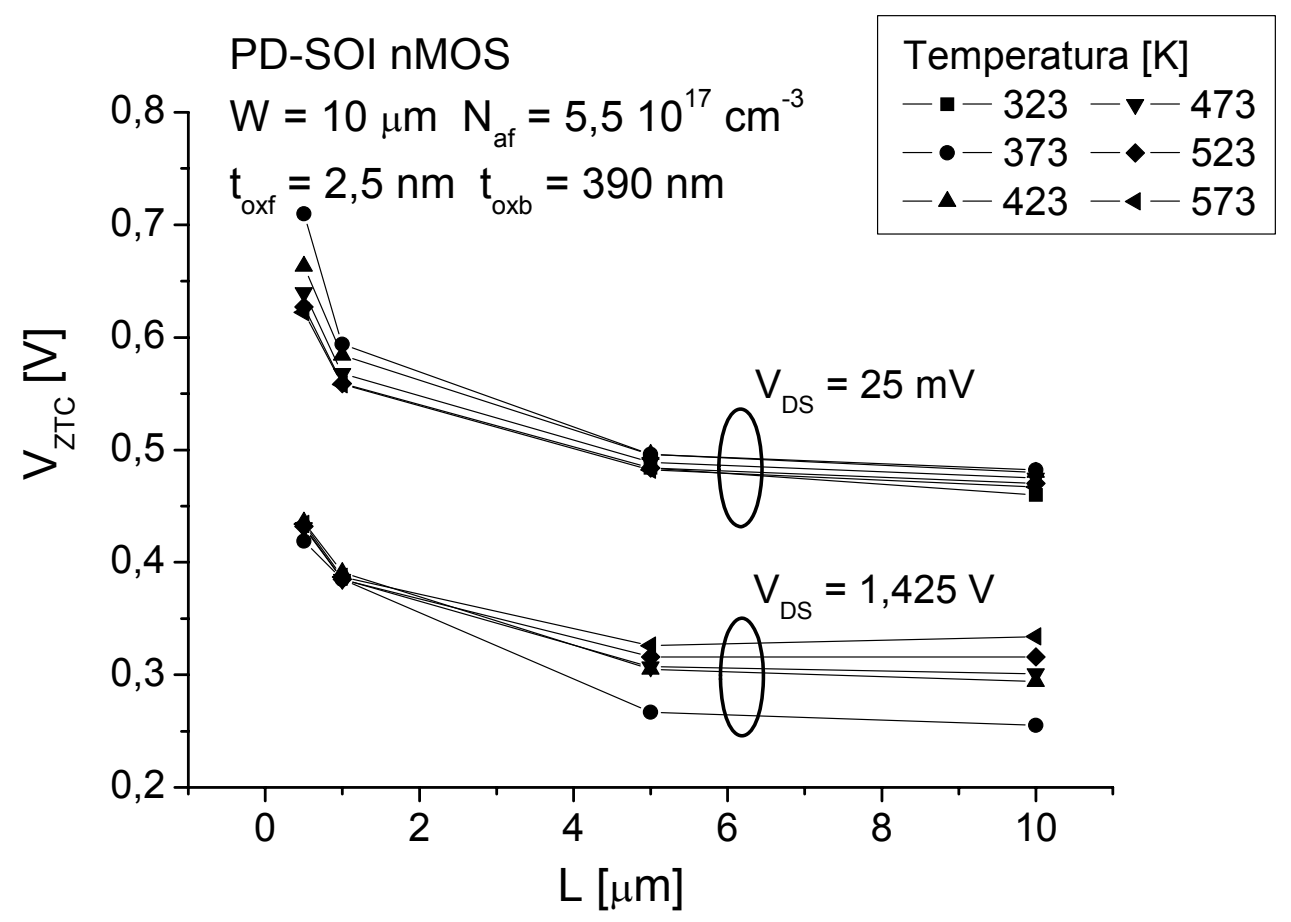

Figura 41: Tensão de polarização no ponto ZTC $\left(V_{Z T C}\right)$ obtido experimentalmente em função do comprimento de canal para dispositivos PD SOI nMOS operando nas regiões linear $\left(V_{D S}=25 \mathrm{mV}\right)$ e de saturação $\left(\mathrm{V}_{D S}=1,425 \mathrm{~V}\right)$, para valores de comprimento de canal. de $0,5,1,5$ e $10 \mu \mathrm{m}$. 


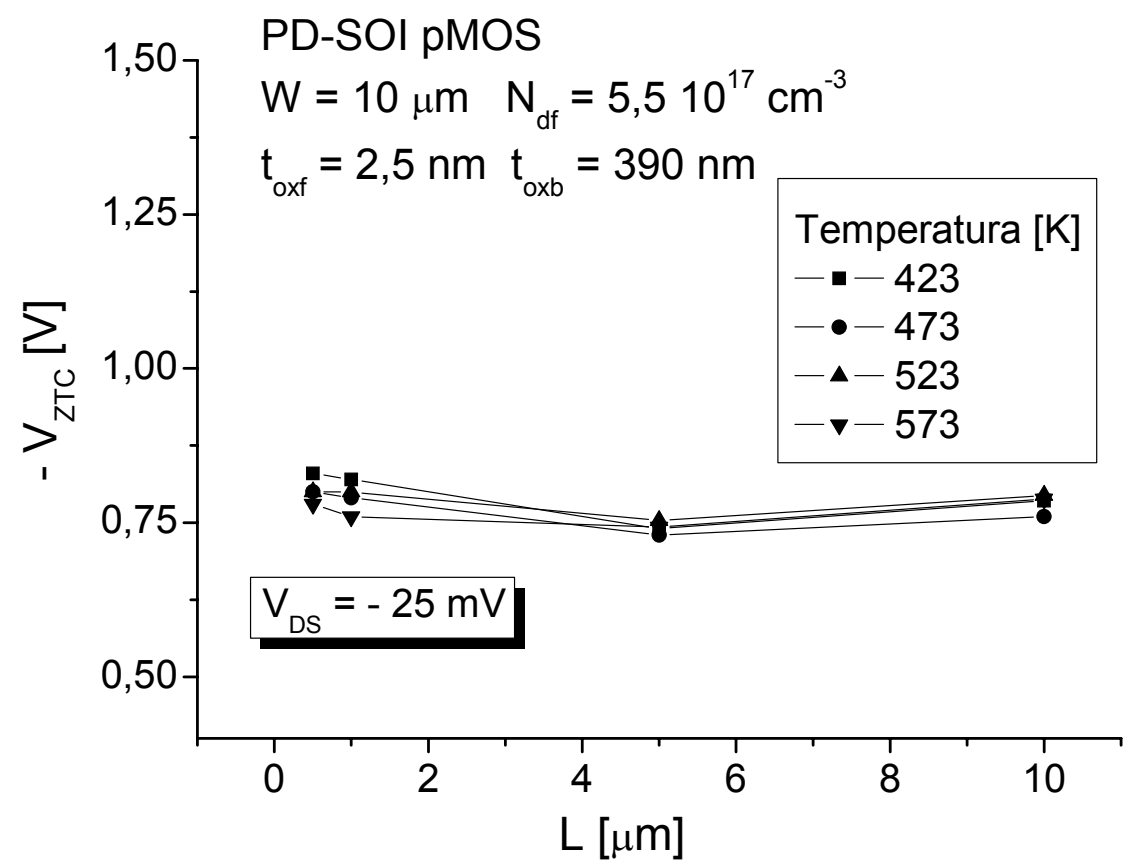

Figura 42: Tensão de polarização no ponto ZTC $\left(\mathrm{V}_{\mathrm{ZTC}}\right)$ em função do comprimento de canal obtido experimentalmente, para dispositivos PD SOI pMOS operando na região linear $\left(\mathrm{V}_{\mathrm{DS}}=\right.$ $25 \mathrm{mV}$ ), para valores de comprimento de canal de 0,5, 1, 5 e $10 \mu \mathrm{m}$.

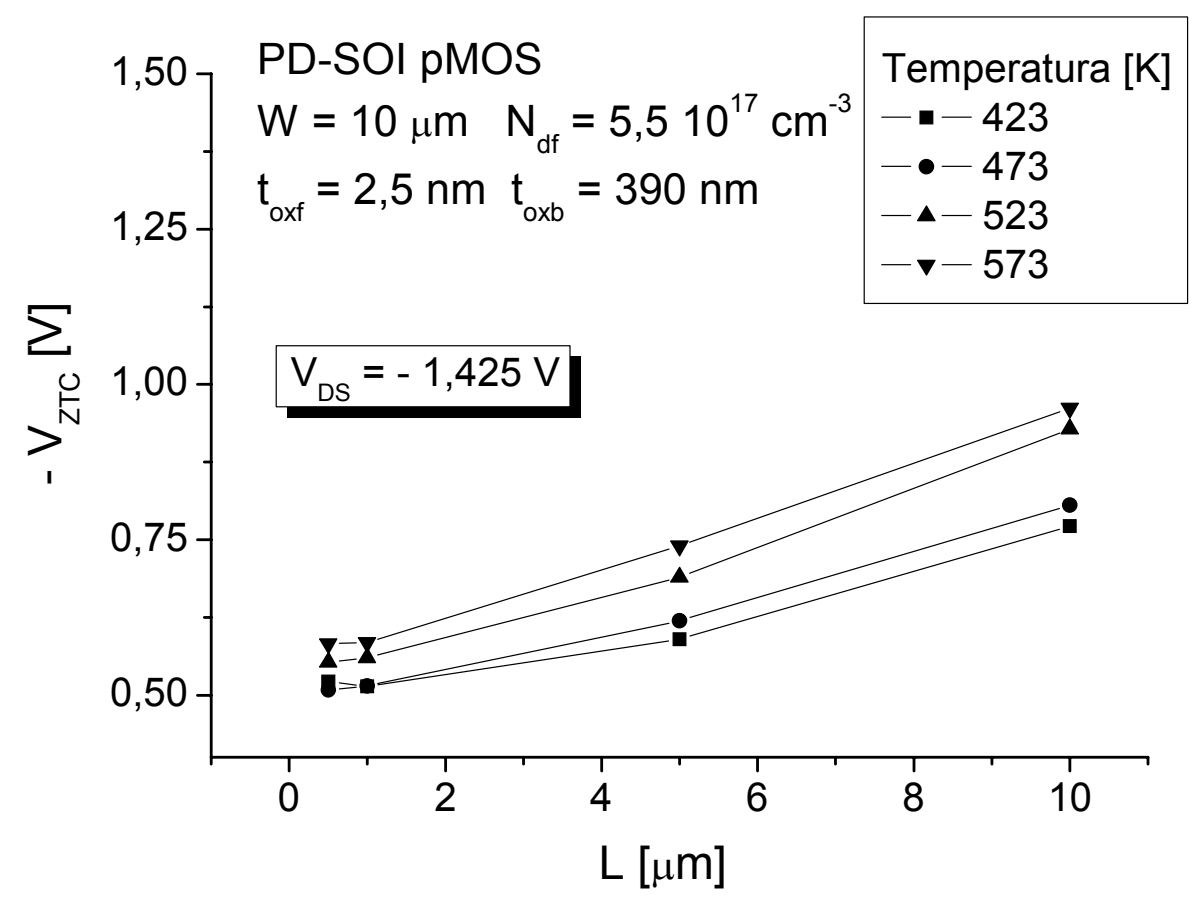

Figura 43: Tensão de polarização no ponto ZTC $\left(\mathrm{V}_{\mathrm{ZTC}}\right)$ em função do comprimento de canal obtido experimentalmente, para dispositivos PD SOI pMOS operando na região de saturação $\left(V_{D S}=1,425 \mathrm{~V}\right)$, para valores de comprimento de canal de 0,5, 1, 5 e $10 \mu \mathrm{m}$. 
Em altas temperaturas, o espalhamento dos portadores devido a rede da estrutura atômica do material degrada a mobilidade dos mesmos, o que leva a redução da corrente de dreno (IDS). Com o aumento da temperatura, ocorre o incremento da concentração intrínseca de portadores $\left(n_{i}\right)$ e a redução do potencial de Fermi $\left(\phi_{F}\right)^{47,61}$. O resultado das mudanças de $n_{i}$ e $\phi_{F}$ com a temperatura causa a redução na largura da região de depleção na camada de silício, e desta forma observa-se a redução da tensão de limiar $\left(\mathrm{V}_{\text {thF }}\right)$ com aumento da temperatura.

\subsection{Influência da dependência da estabilidade do ponto ZTC com a Tensão de Dreno ( $V_{D S}$ )}

Nesse item é estudada a influência da variação da tensão de dreno $\left(\mathrm{V}_{\mathrm{DS}}\right)$ no ponto invariante com a temperatura $\left(\mathrm{V}_{\mathrm{ZTC}}\right)$, utilizando-se do modelo CM proposto no capítulo 4 e os dados obtidos experimentalmente, para dispositivos SOI n e p MOSFETs operando em regime parcialmente (PD) depletado.

\subsubsection{Características físicas dos dispositivos}

As estruturas dos transistores usadas nesta etapa foram as mesmas da etapa 5.2.

\subsubsection{Resultados e Discussão}

Para o estudo do efeito da variação da tensão de dreno ( $\left.\bigvee_{D S}\right)$ na tensão de polarização no ponto ZTC $\left(\mathrm{V}_{\mathrm{ZTC}}\right)$, foram analisados os dois parâmetros que influênciam diretamente na mudança em seu valor. 
Na Figura 44 e na Figura 45 pode-se observar a influência da variação na tensão de dreno $\left(\mathrm{V}_{\mathrm{DS}}\right)$ sobre o valor da tensão de limiar $\left(\mathrm{V}_{\mathrm{thF}}\right) \mathrm{e}$ na mobilidade dos portadores do canal $\left(\mu_{n}\right)$, respectivamente. A tensão $V_{\text {thF }}$ apresentou redução com o aumento de $\mathrm{V}_{\mathrm{DS}}$, para toda a faixa analisada de 0 a 1,425 V. A redução significativa no valor da mobilidade dos portadores do canal $\mu_{\mathrm{n}}$ ocorreu para valores de $\mathrm{V}_{\mathrm{DS}}$ entre 0 e $0,4 \mathrm{~V}$.

Com a redução em $V_{\text {thF }}$ e $\mu_{n}$, foi observada a diminuição do valor da tensão de polarização no ponto ZTC ( $\left.\mathrm{V}_{\text {ZTC }}\right)$ para uma polarização de tensão de dreno alta, acima de $1 \mathrm{~V}$. A variação na tensão de limiar $\left(\Delta \mathrm{V}_{\text {thF }}\right)$ pode ser expressa $^{62}$ em função de $V_{D S}$. A redução da mobilidade dos portadores do canal, devido a saturação da velocidade desses portadores na presença de um alto campo elétrico longitudinal $\left(\mathrm{V}_{\mathrm{DS}}\right)$, foi modelada ${ }^{63}$ pelo parâmetro da degradação efetiva da mobilidade. O efeito da tensão de dreno em $V_{\text {ZTC }}$ é mostrado na Figura 46 e na Figura 47 para dispositivos nMOS e pMOS, respectivamente.

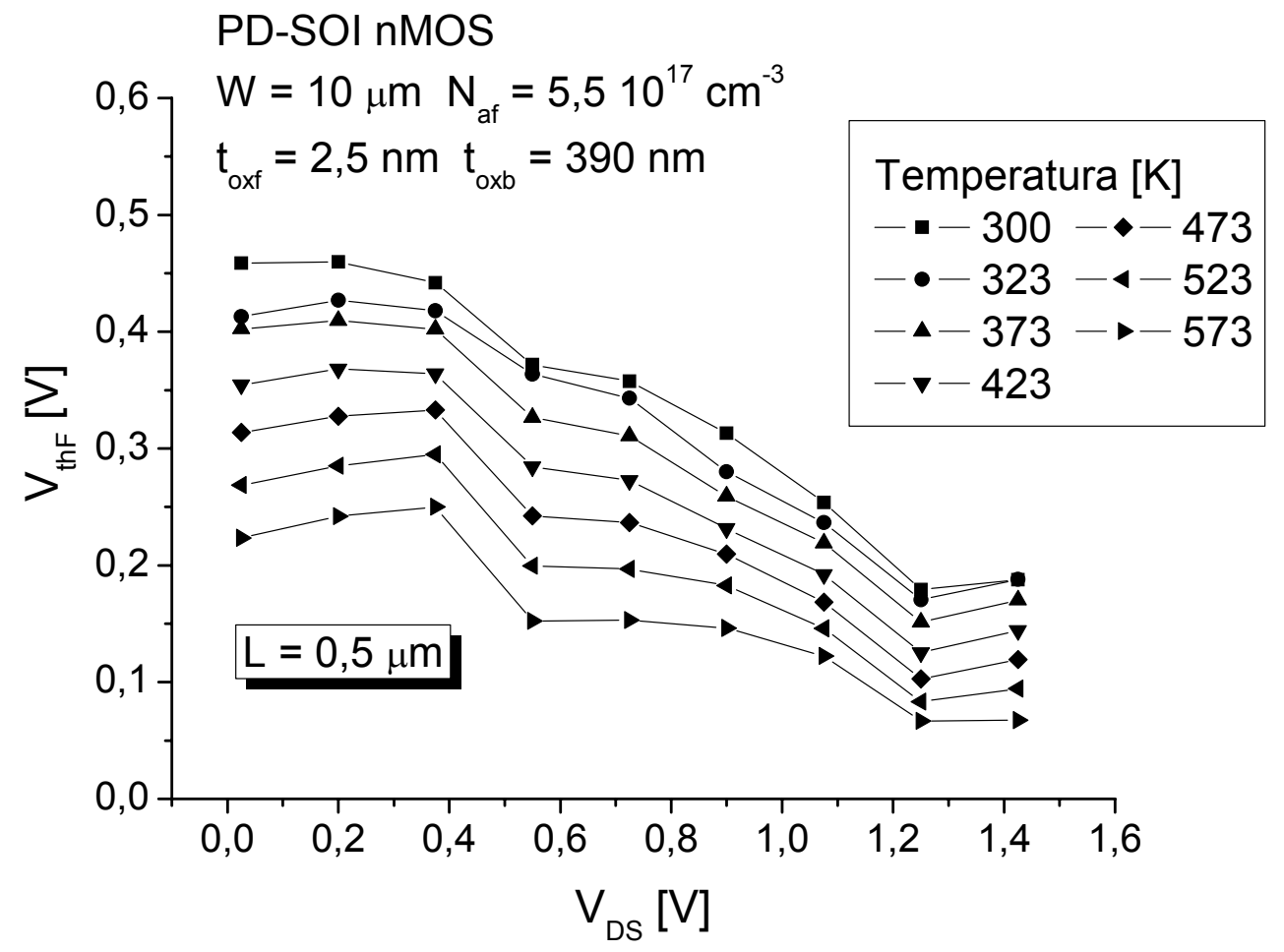


Figura 44: Dados experimentais: Tensão de limiar $\left(\mathrm{V}_{\mathrm{thF}}\right)$ em função da tensão de dreno $\left(V_{D S}\right)$ para dispositivos PD SOI nMOS, para temperaturas de 300 a $573 \mathrm{~K}$.

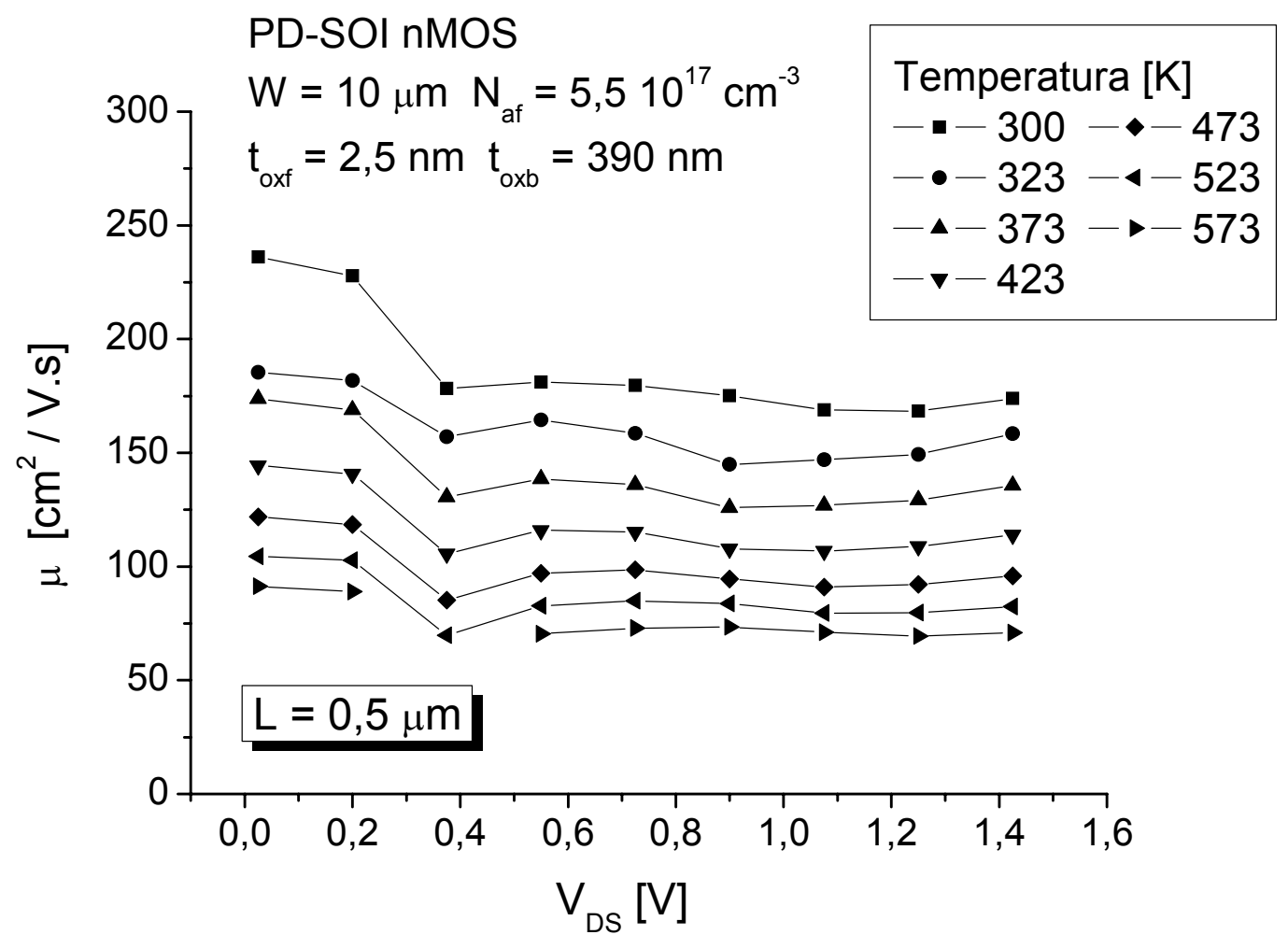

Figura 45: Dados experimentais: Mobilidade dos portadores no canal $\left(\mu_{n}\right)$ em função da tensão de dreno $\left(\mathrm{V}_{\mathrm{DS}}\right)$ para dispositivos PD SOI nMOS, para temperaturas de 300 a $573 \mathrm{~K}$.

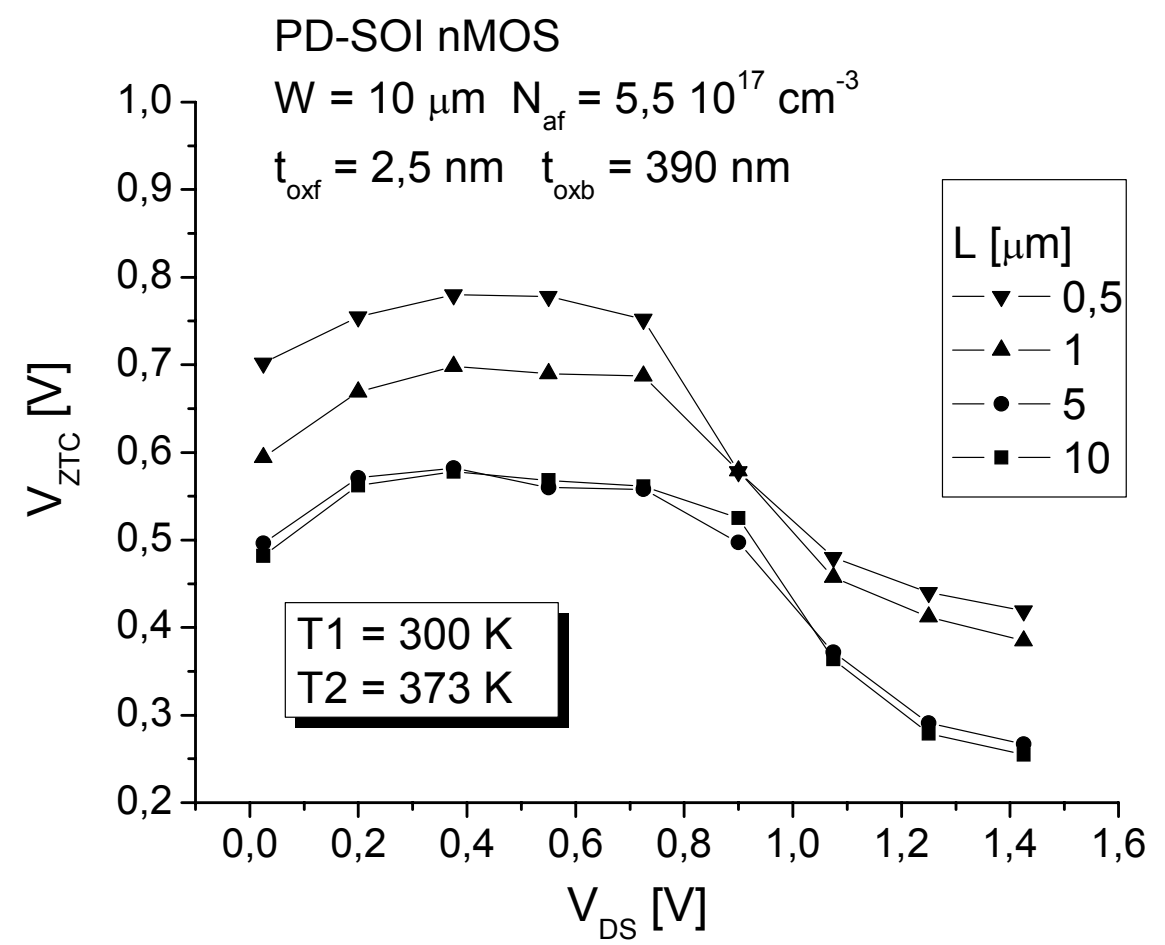


Figura 46: Tensão de polarização no ponto $Z T C\left(V_{Z T C}\right)$ em função da tensão de dreno $\left(V_{D S}\right)$, obtida experimentalmente para dispositivos PD SOI nMOS com comprimentos de canal (L) de $0,5,1,5$ e $10 \mu \mathrm{m}$.

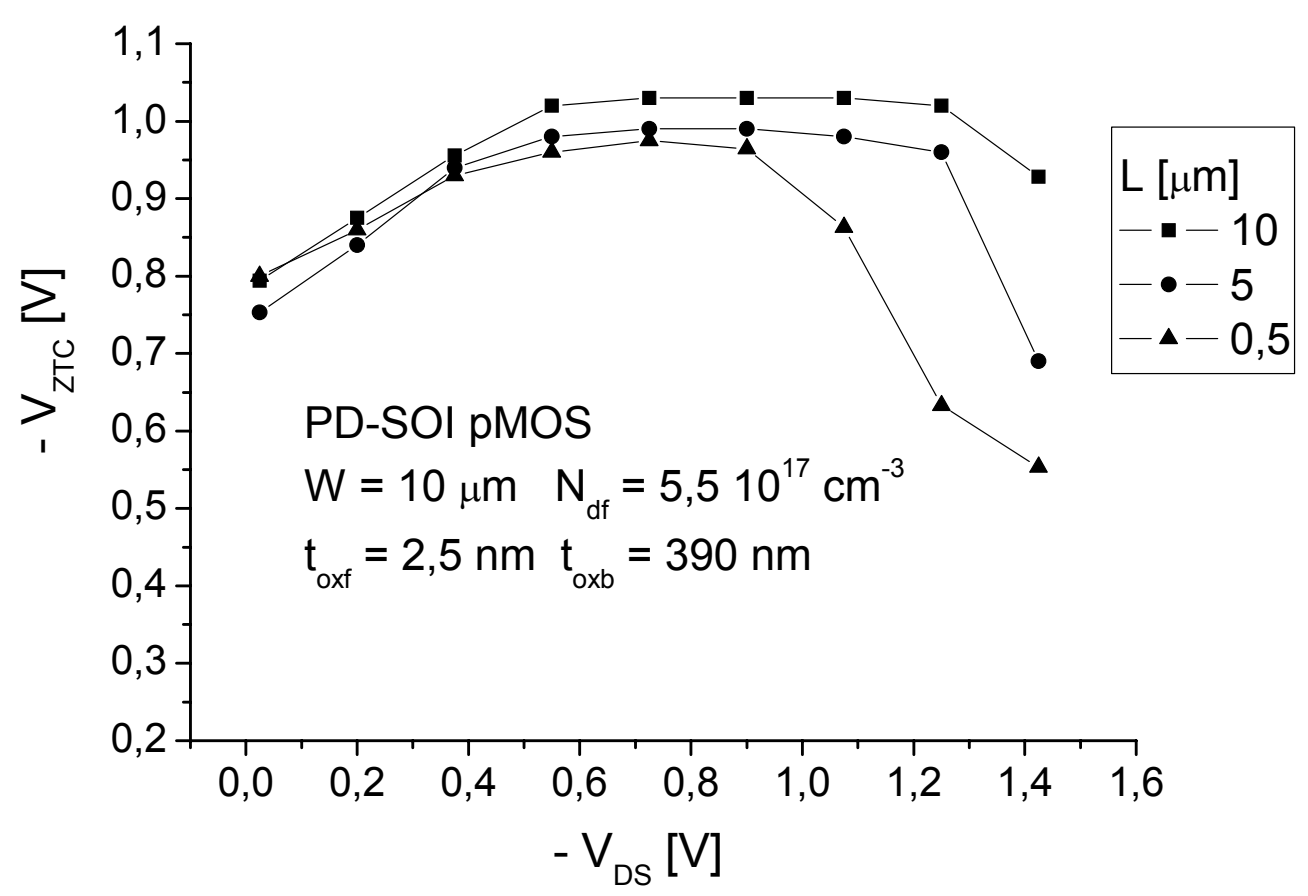

Figura 47: Tensão de polarização no ponto ZTC $\left(V_{Z T C}\right)$ em função da tensão de dreno $\left(V_{D S}\right)$, obtida experimentalmente para dispositivos PD SOI pMOS com comprimentos de canal (L) de $0,5,5$ e $10 \mu \mathrm{m}$.

A redução observada em $V_{\text {ZTC }}$ para um alto valor da tensão de dreno $\left(V_{D S}>1.0 \mathrm{~V}\right)$ possivelmente devido ao efeito DIBL (drain-induced-barrierlowering) sobre a tensão de limiar $\left(\mathrm{V}_{\mathrm{thF}}\right)$, uma vez que a barreira de potencial para formar o canal de condução é controlada por ambas as polarizações da tensão de porta $\left(\mathrm{V}_{\mathrm{GF}}\right)$ e a tensão de dreno $\left(\mathrm{V}_{\mathrm{DS}}\right)$. Quando $\mathrm{V}_{\mathrm{DS}}$ aumenta, a barreira de potencial diminui, levando a ocorrência do efeito DIBL, que causa possivelmente a queda no valor da tensão de $\operatorname{limiar}^{14,62}$. Como conseqüência, ocorre um menor valor da tensão de polarização no ponto ZTC $\left(V_{\text {ZTC }}\right)$ para tensão de dreno alta em dispositivos SOI MOSFET ${ }^{47}$.

O modelo CM proposto, através das equações $47,51,54$ e 55, mostra claramente que quando $V_{\text {th1 }}$ diminui, $V_{\text {ZTC }}$ também irá diminuir e que está de 
acordo com os resultados experimentais obtidos, mostrados nas figuras Figura 46 e Figura 47.

\subsection{Aplicação do modelo CM proposto em outras tecnologias}

Neste item é apresentada a aplicação e comparação do modelo CM proposto do ponto ZTC, com dados obtidos de dispositivos fabricados em outras tecnologias.

A primeira análise foi realizada com a estrutura SOI MOSFET, com perfil de dopantes assimétrico na região do canal, denominado GC SOI MOSFET, que foi desenvolvida com o objetivo de aumentar a tensão de ruptura, diminuir o efeito bipolar parasitário e melhorar a condutância de saída (maior tensão Early) ${ }^{64}$. A segunda análise foi realizada com dispositivos que foram fabricados em estrutura SOI de porta circundante com canal gradual (GCGAA SOI ${ }^{65}$ - "Gate-All-Around Graded Channel SOI"). Nos transistores GAA $\mathrm{SOI}^{66}$ a camada de silício é envolvida pelo óxido de porta e pelo material de porta em toda região do canal, Figura 48A. Estes dispositivos são bastante atrativos para aplicações de baixa tensão e baixa potência, apresentam uma alta resistência à ambientes desfavoráveis, tais como altas temperaturas e ambiente com radiação ${ }^{67}$.

A estrutura SOI de porta circundante com canal gradual (GC-GAA) nada mais é do que a combinação dos resultados excelentes obtidos com o transistor GC SOI, a partir do perfil de dopantes na região do canal, com as melhorias promovidas nos transistores GAA, devido à presença de dois canais de inversão. A região do canal deste dispositivo é dividida em duas partes: uma com a concentração de dopantes natural da lâmina junto ao dreno $\left(L_{L D}\right)$ e a segunda com a concentração de dopantes usualmente utilizada para ajuste da tensão de limiar de transistores SOI totalmente depletados, junto à fonte $\left(L_{H D}\right)$, a Figura $48 \mathrm{~B}$ mostra um exemplo de um transistor nMOS com as regiões do canal $P+\left(L_{H D}\right)$ e $P-\left(L_{L D}\right)$. 
A)

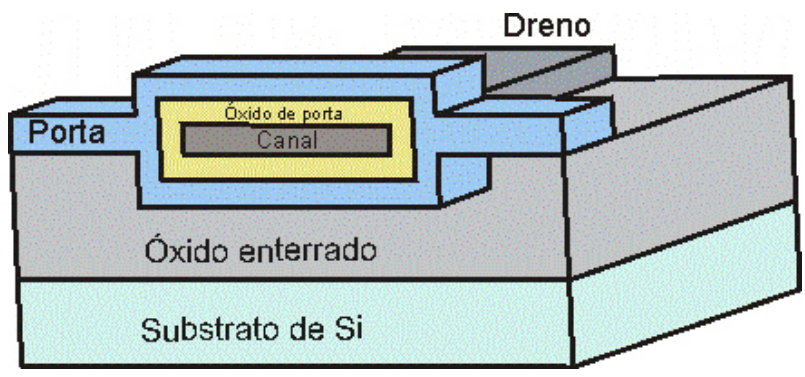

B)

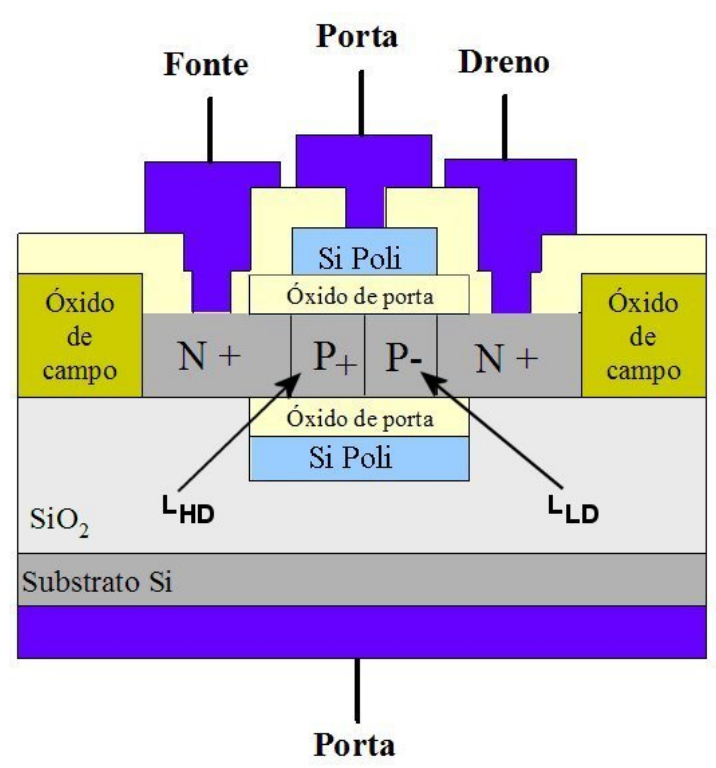

Figura 48 Perfis da estrutura GC GAA SOI ${ }^{65}$ : Vista em perspectiva (A) e Corte longitundinal (B)

\subsubsection{Análise do modelo CM proposto em dispositivos GC SOI}

\subsubsection{Características dos Dispositivos}

Os dispositivos GC SOI analisados possuem espessura do óxido de porta ( $\left.t_{\text {oxf }}\right)$ de $31 \mathrm{~nm}$, espessura do óxido enterrado ( $\left.t_{\text {oxb }}\right)$ de $390 \mathrm{~nm}$ e $80 \mathrm{~nm}$ a espessura da camada de silício $\left(\mathrm{t}_{\mathrm{Si}}\right)$. Nos dispositivos GC SOI a concentração da região de baixa dopagem do canal é $\mathrm{Nab}=1.10^{15} \mathrm{~cm}^{-3}$, sendo esta a mesma concentração do substrato. No GC SOI a concentração da região que sofreu implantação para o ajuste da tensão de limiar é $\mathrm{N}_{\mathrm{af}}=$ $1.10^{17} \mathrm{~cm}^{-3}$, sendo esta a mesma concentração de todo o canal nos 
dispositivos SOI convencionais, utilizados como referência para o valor total do comprimento de canal.

As curvas foram extraídas variando-se a temperatura, no intervalo entre 27 a $300{ }^{\circ} \mathrm{C}(300 \mathrm{~K}$ a $573 \mathrm{~K})$, tanto para o SOl convencional como para o GC SOI totalmente depletados com relação $L_{L D} / L$ de $0,25,0,5$ e 0,825. Todos os dispositivos possuem $L=4 \mu \mathrm{m}$ e $\mathrm{W}=18 \mu \mathrm{m}$ (W sendo a largura do canal).

\subsubsection{Resultados e Discussão}

A análise dos resultados, para os dispositivos GC SOI, será conduzida apresentando uma seqüência de análise dos parâmetros de dados experimentais e obtidos pelo modelo $\mathrm{CM}$ proposto para a tensão no ponto invariante com a temperatura $\left(\mathrm{V}_{\mathrm{ZTC}}\right)$, em ambos os regimes de polarização do transistor, ou seja, linear e saturação.

\subsection{Ocorrência do Ponto Invariante com a Temperatura ( $\left.\mathrm{V}_{\mathrm{ZTC}}\right)$}

A primeira verificação realizada foi através das curvas $I_{D S} \times V_{G F}$ obtidas através de medidas experimentais com os transistores GC SOI nMOSFETs, operando nas regiões linear e de saturação e em diferentes temperaturas. Foi possível observar a ocorrência do ponto ZTC para os diferentes valores da relação de comprimento de canal $\left(\mathrm{L}_{\mathrm{LD}} / \mathrm{L}\right)$, e que são apresentadas para as relações $L_{L D} / L$ de 0,25 e 0,5 em ambas as condições de polarização, Figura 49 a Figura 52. 


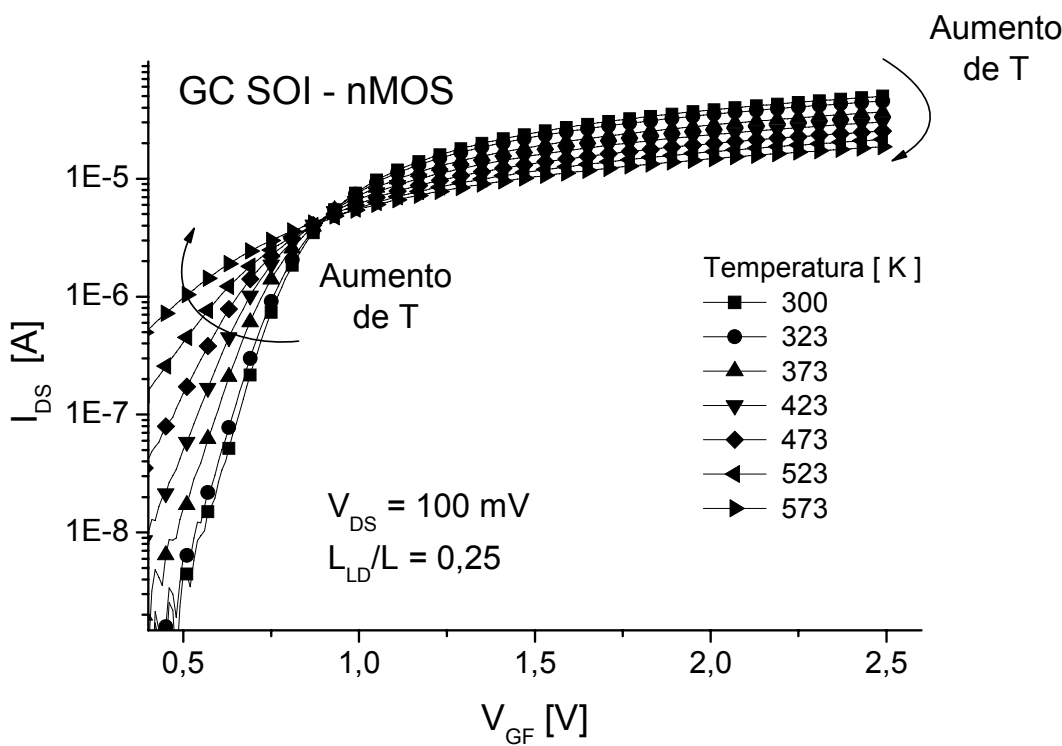

Figura 49: Curvas $I_{D S} \times V_{G F}$ de medidas experimentais do transistor GC SOI nMOS $\left(L_{L D} / L=0,25\right)$ em diferentes temperaturas para $V_{D S}=100 \mathrm{mV}$.

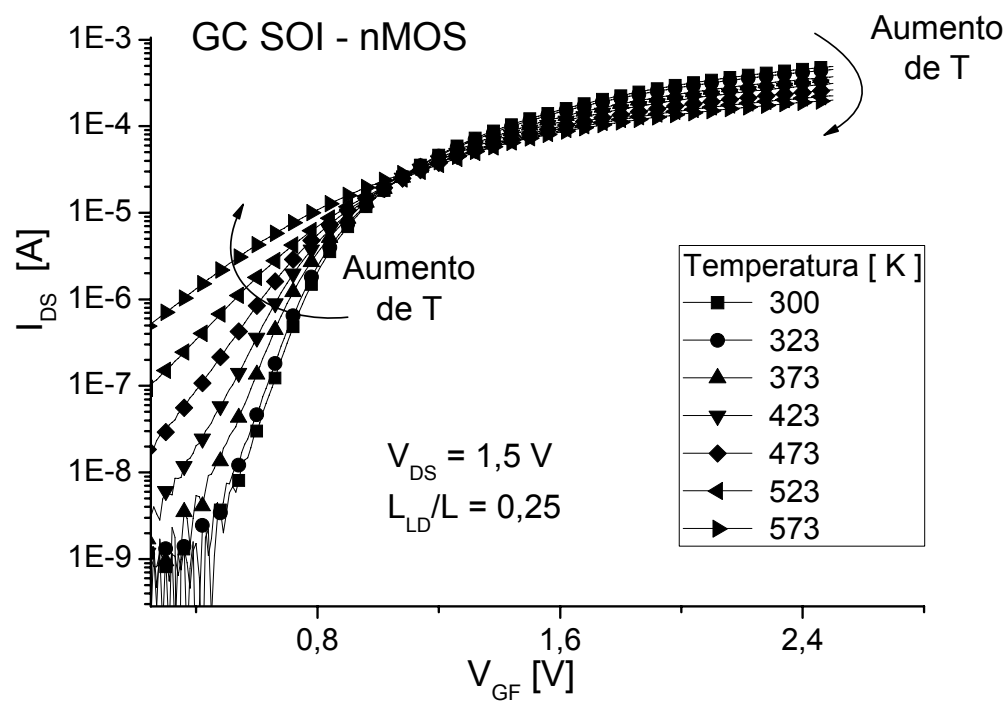

Figura 50: Curvas $I_{D S} \times V_{G F}$ de medidas experimentais do transistor GC SOI nMOS $\left(\mathrm{L}_{\mathrm{LD}} / \mathrm{L}=0,25\right)$ em diferentes temperaturas para $\mathrm{V}_{\mathrm{DS}}=1,5 \mathrm{~V}$. 


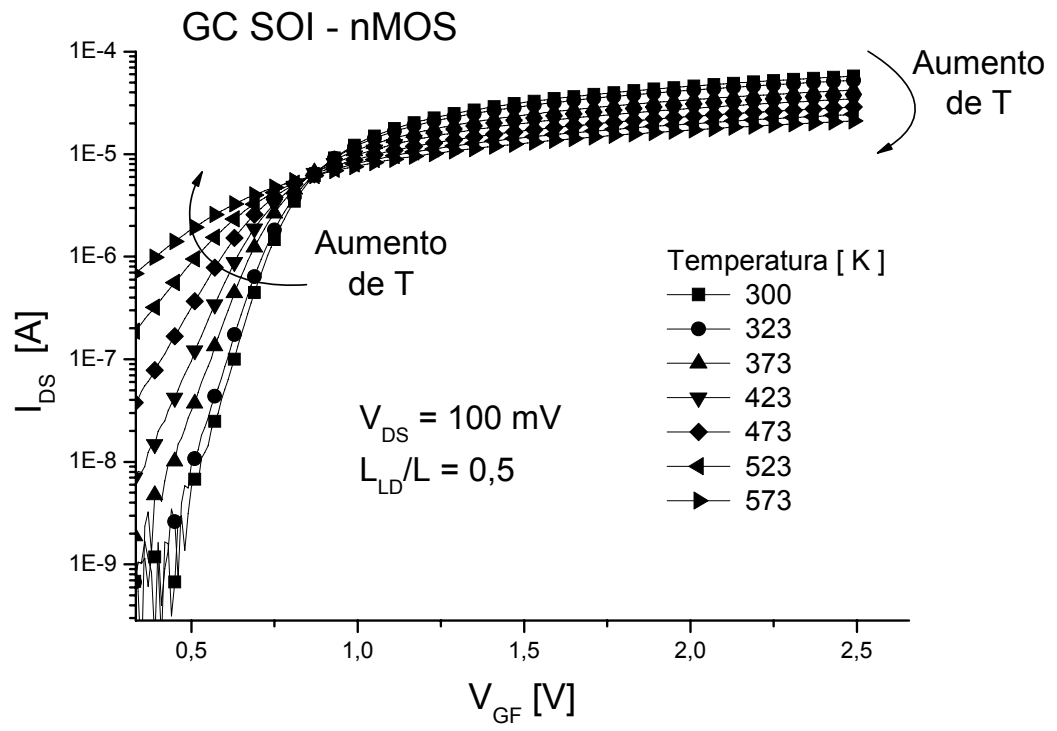

Figura 51: Curvas $I_{D S} \times V_{G F}$ de medidas experimentais do transistor GC SOI nMOS $\left(L_{\mathrm{LD}} / \mathrm{L}=0,5\right)$ em diferentes temperaturas para $V_{\mathrm{DS}}=100 \mathrm{mV}$.

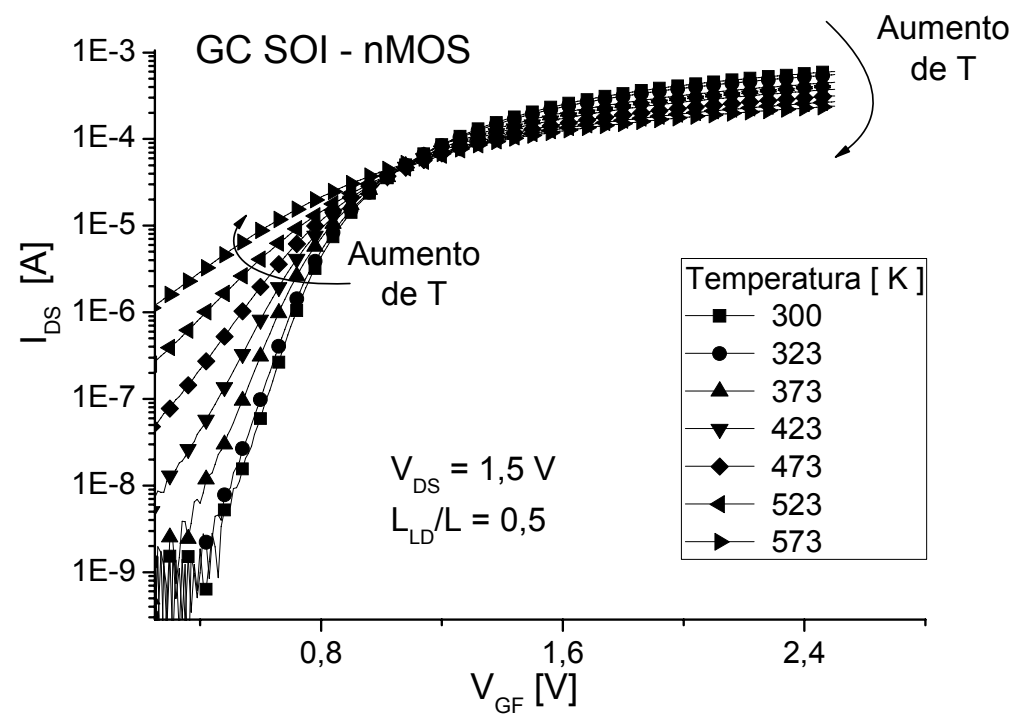

Figura 52: Curvas $I_{D S} \times V_{G F}$ de medidas experimentais do transistor GC SOI nMOS $\left(L_{L D} / L=0,5\right)$ em diferentes temperaturas para $V_{D S}=1,5 \mathrm{~V}$. 


\subsubsection{2 $\mathrm{V}_{\mathrm{ZTC}}$ : Dados Experimentais e Modelo CM}

Nas figuras a seguir é apresentado o valor de $\mathrm{V}_{\mathrm{ZTC}}$ em função da temperatura, que foi obtido experimentalmente e pelo modelo simples proposto, operando na região linear, através da equação (47), e na região de saturação, utilizando a equação (51). Os valores obtidos para os dispositivos GC SOI são para as relações de $L_{L D} / L\left(L_{H D}\right)$ de $0,25(3 \mu \mathrm{m}), 0,5$ $(2 \mu \mathrm{m})$ e para o dispositivo SOI convencional com $L=4 \mu \mathrm{m}$, para ambas as polarizações, da Figura 53 a Figura 55 . E com $\mathrm{L}_{\mathrm{LD}} / \mathrm{L}\left(\mathrm{L}_{\mathrm{HD}}\right)$ de $0,825(0,7 \mu \mathrm{m})$ na região linear, Figura 56 , e $L_{L D} / L\left(L_{H D}\right)$ de $0,625(1,5 \mu \mathrm{m})$ na região de saturação, Figura 57.

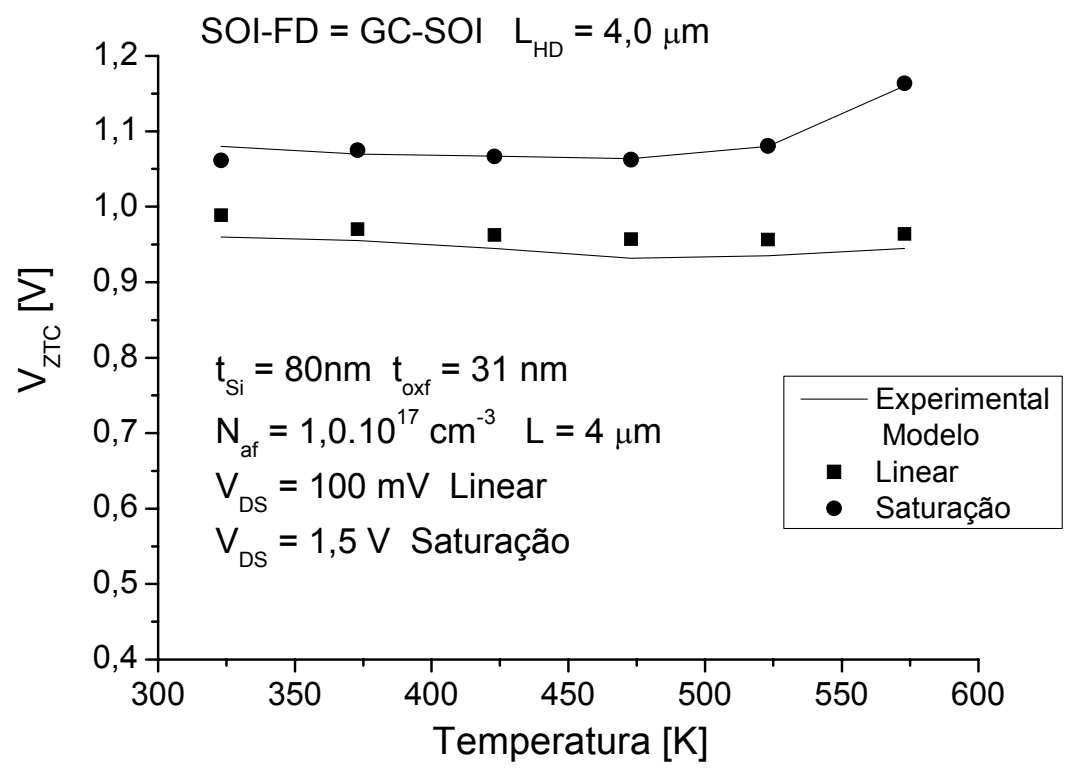

Figura 53: Tensão $V_{\mathrm{ZTC}}$ obtido experimentalmente e pelo modelo proposto em função da temperatura para dispositivos SOI nMOS Convencionais, para as duas regiões de operação linear $\left(V_{D S}=100 \mathrm{mV}\right)$ e de saturação $\left(V_{D S}=1,5 \mathrm{~V}\right)$, comprimento de canal de $L=4 \mu \mathrm{m}$. 


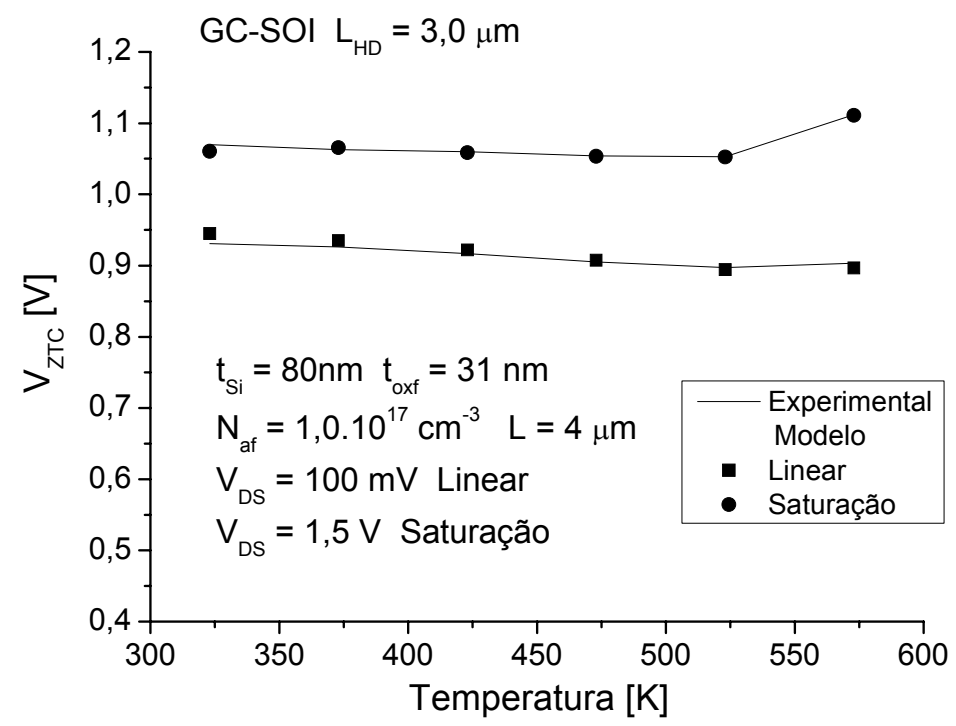

Figura 54: Tensão $V_{\text {ZTC }}$ obtido experimentalmente e pelo modelo proposto em função da temperatura para dispositivos GC SOI nMOS, para as duas regiões de operação linear $\left(V_{D S}=100 \mathrm{mV}\right)$ e de saturação $\left(V_{D S}=1,5 \mathrm{~V}\right)$, para relação de comprimento de canal $\mathrm{L}_{\mathrm{LD}} / \mathrm{L}=0,25$ ou $\mathrm{L}_{H D}=3 \mu \mathrm{m}$.

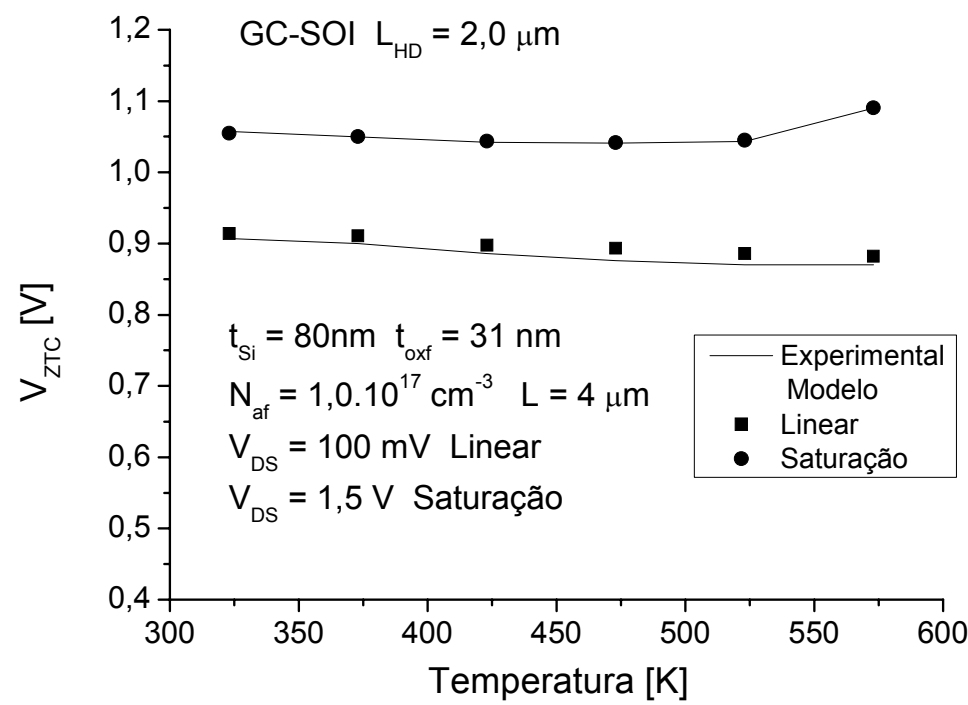

Figura 55: Tensão $V_{\text {ZTC }}$ obtido experimentalmente e pelo modelo proposto em função da temperatura para dispositivos GC SOI nMOS, para as duas regiões de operação linear ( $V_{D S}$ $=100 \mathrm{mV})$ e de saturação $\left(\mathrm{V}_{\mathrm{DS}}=1,5 \mathrm{~V}\right)$, para relação de comprimento de canal $\mathrm{L}_{\mathrm{LD}} / \mathrm{L}=0,5$ ou $L_{H D}=2 \mu \mathrm{m}$. 


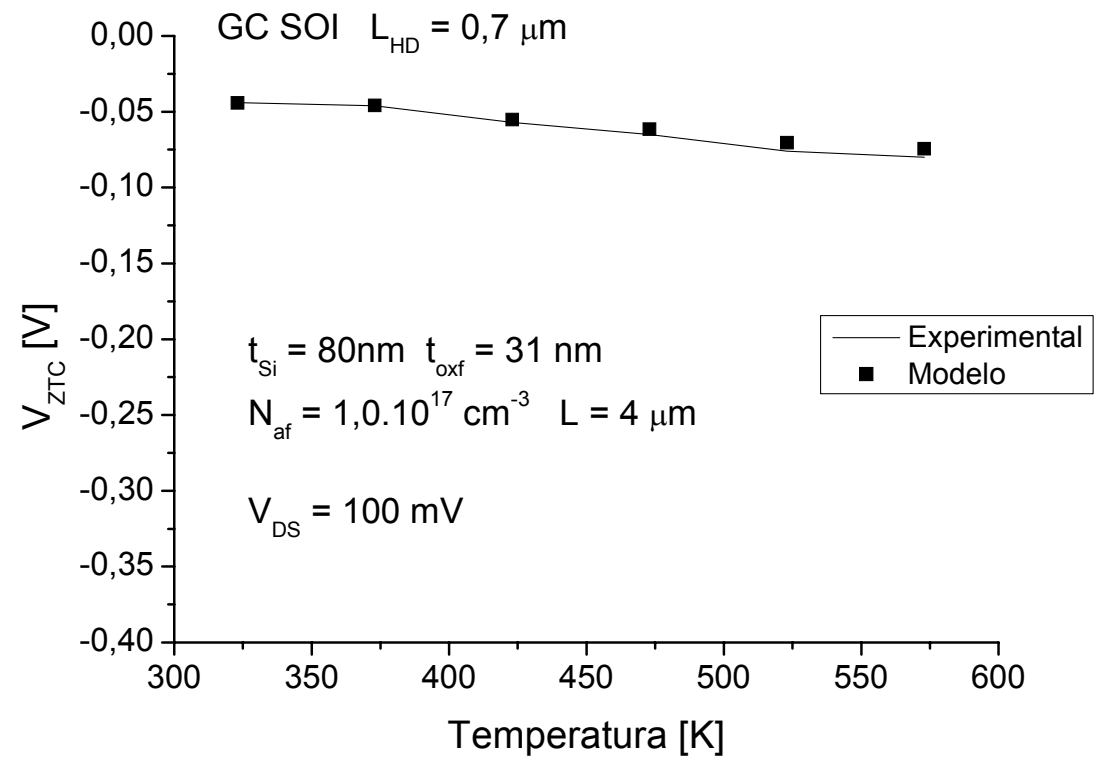

Figura 56: Tensão $\mathrm{V}_{\mathrm{ZTC}}$ obtido experimentalmente e pelo modelo proposto em função da temperatura para dispositivos GC SOI nMOS, operando na região linear $\left(\mathrm{V}_{\mathrm{DS}}=100 \mathrm{mV}\right)$, para comprimento de canal $L_{H D}=0,7 \mu \mathrm{m}$.

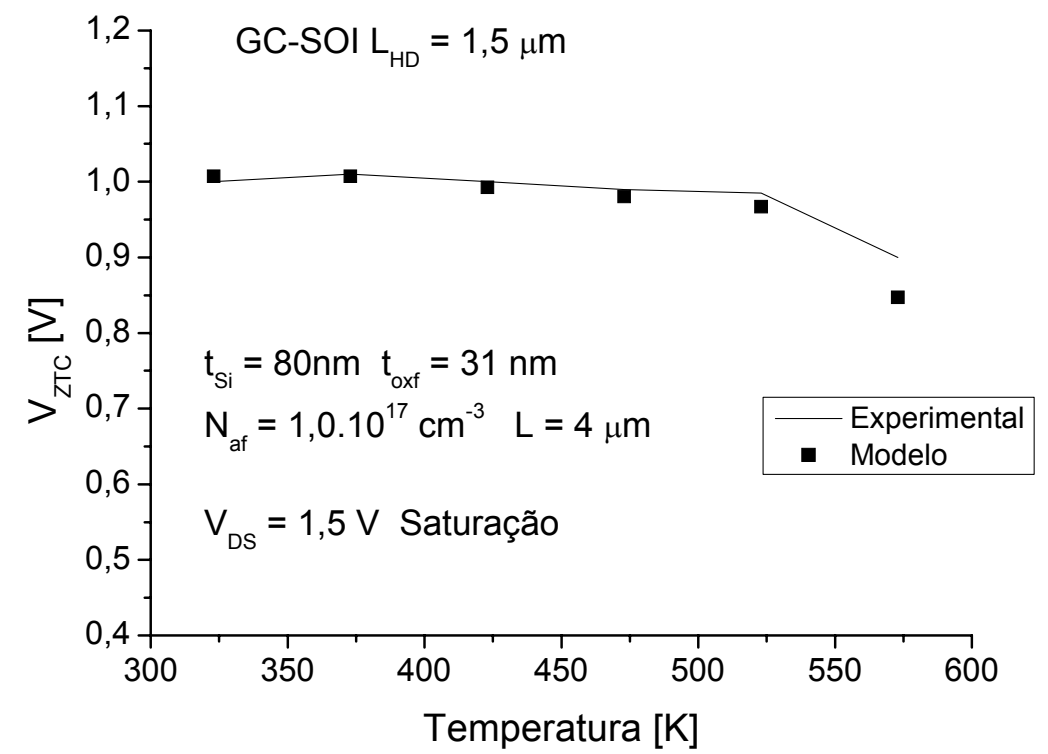

Figura 57: Tensão $V_{\text {ZTC }}$ obtido experimentalmente e pelo modelo proposto em função da temperatura para dispositivos GC SOI nMOS, operando na região de saturação $\left(V_{D S}=1,5 \mathrm{~V}\right)$, para comprimento de canal $\mathrm{L}_{\mathrm{HD}}=1,5 \mu \mathrm{m}$. 
Os valores do fator $c$, que representa a degradação da mobilidade com a temperatura, que foram utilizados para o ajuste dos resultados do modelo proposto aos dados experimentais, são apresentados na Tabela 5 para cada dispositivo. $O$ valor do fator $c$ foi obtido através da equação (46) aplicada aos dados experimentais, e adotado a média aritmética do fator $c$ dos valores para a faixa de temperatura analisada, 300 a $573 \mathrm{~K}$. Com a utilização dos valores encontrados do fator c na equação (47), foi observado um bom ajuste dos resultados do modelo proposto com os dados experimentais, apesar das simplificações e considerações adotadas.

Os valores do fator $\mathrm{c}$ em função do comprimento de canal $\mathrm{L}_{\mathrm{HD}}$, Tabela 5 , indicam a maior degradação da mobilidade com o aumento da temperatura em dispositivos com menor comprimento de canal $\left(\mathrm{L}_{\mathrm{HD}}\right)$. Há também a ocorrência de uma maior degradação da mobilidade para menores comprimentos de canal devido o aumento do campo elétrico ${ }^{68}$, uma vez que em um escalamento realista de dispositivos, a tensão elétrica é reduzida com um fator de escala menor do que o utilizado para as dimensões. Isto faz com que o campo elétrico aumente com o escalamento.

Tabela 5: Média aritmética do valor do fator c para cada comprimento de canal $L_{H D}$, para faixa de temperatura analisada, 300 a $573 \mathrm{~K}$.

\begin{tabular}{|c|c|c|c|c|c|}
\hline \multicolumn{7}{|c|}{ Fator c (média) } \\
\hline LHD $[\mu \mathrm{m}]$ & 4 & 3 & 2 & 1,5 & 0,7 \\
\hline Linear & $\mathbf{1 , 4 6}$ & $\mathbf{1 , 6 5}$ & $\mathbf{1 , 8 1}$ & & $\mathbf{1 , 9 1}$ \\
\hline Saturação & $\mathbf{1 , 5 9}$ & $\mathbf{1 , 6 2}$ & $\mathbf{1 , 8 8}$ & $\mathbf{2 , 4 7}$ & \\
\hline
\end{tabular}




\subsection{Variação de $\mathrm{V}_{\mathrm{ZTC}}$ com comprimento de canal (L)}

A influência no valor do ponto invariante com a temperatura ( $\left.\mathrm{V}_{\mathrm{ZTC}}\right)$ com a variação do comprimento de canal do dispositivo, operando em regime linear, Figura 58, e no regime de saturação, Figura 59.

A Figura 58 apresenta 0 valor de $\mathrm{V}_{\text {ZTC }}$, de dados obtidos experimentalmente para os dispositivos GC SOI nMOSFETs, em função do comprimento de canal $L_{H D}$ de 0,7 , 2 e $3 \mu \mathrm{m}$, ou seja, para as relações de comprimento de canal $\left(L_{L D} / L\right)$ de $0,825,0,5$ e 0,25 , respectivamente, e para o dispositivo SOI MOSFET convencional.

Pode-se observar, através dos dados apresentados, a diminuição no valor de $\mathrm{V}_{\mathrm{ZTC}}$ com a redução do comprimento de canal, indicando a sua dependência direta com a tensão de limiar, esta ultima que sofre redução devido a ocorrência do efeito de canal curto. Na Figura 59 também é possível observar a redução de $\mathrm{V}_{\text {ZTC }}$ para menores valores do comprimento de canal $\left(\mathrm{L}_{\mathrm{HD}}\right)$, para os dispositivos operando na região de saturação.

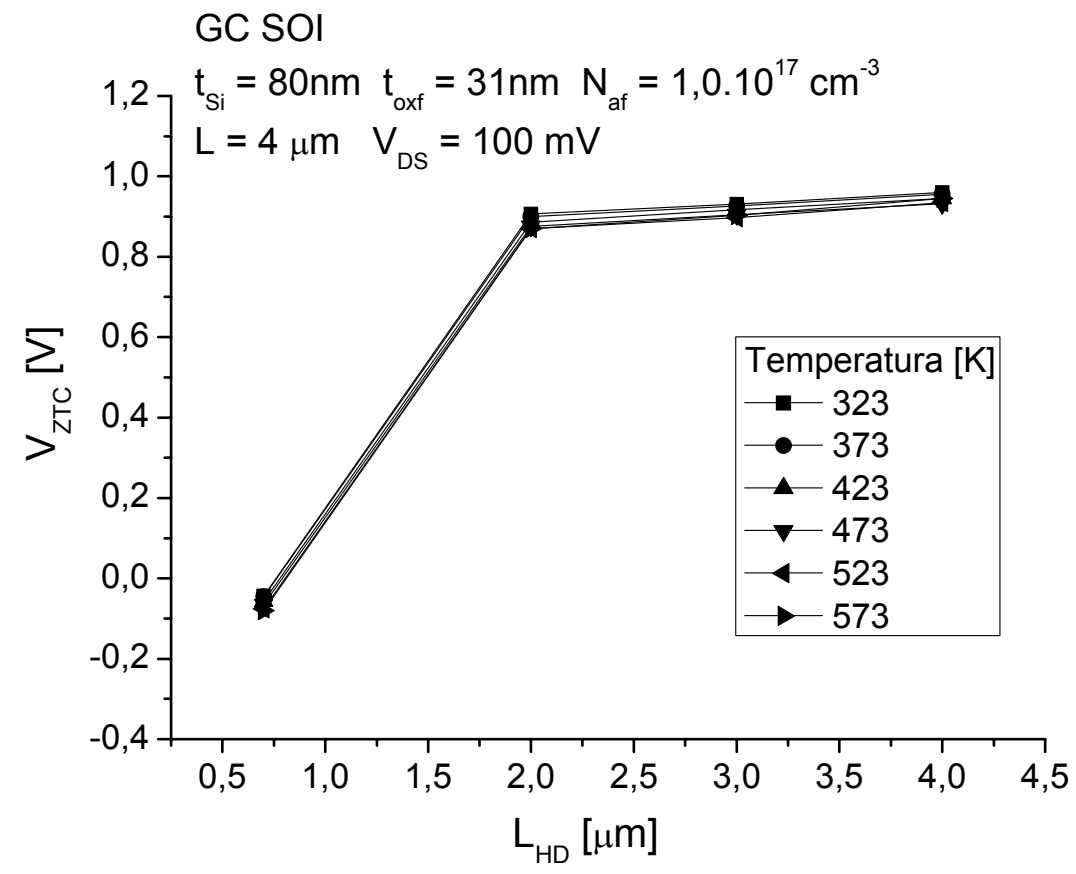

Figura 58: Tensão $V_{Z T C}$ em função do comprimento de canal da região altamente dopada $\left(\mathrm{L}_{\mathrm{HD}}\right)$ obtido experimentalmente, para dispositivos GC SOI nMOS operando na região linear $\left(\mathrm{V}_{\mathrm{DS}}=100 \mathrm{mV}\right)$. 


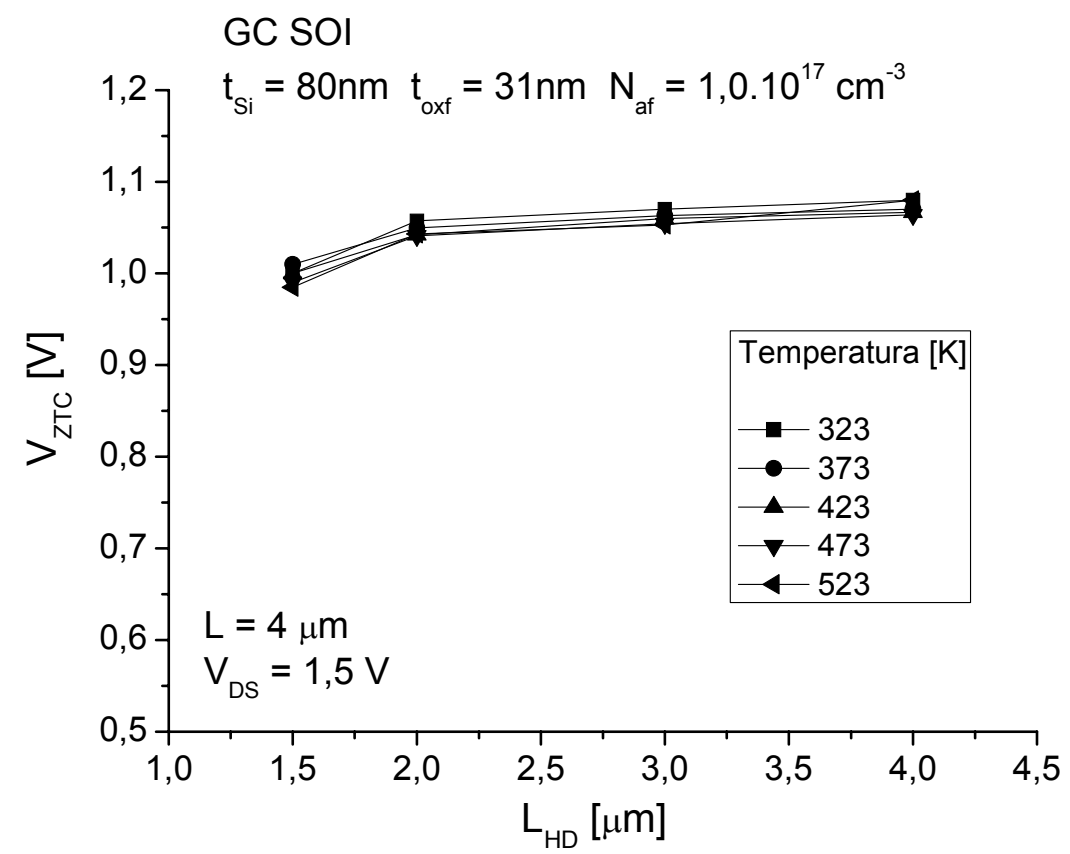

Figura 59: Tensão $V_{\text {ZTC }}$ em função do comprimento de canal da região altamente dopada $\left(L_{H D}\right)$ obtido experimentalmente, para dispositivos GC SOI nMOS operando na região de saturação $\left(V_{D S}=1,5 \mathrm{~V}\right)$.

\subsubsection{Análise do modelo CM proposto em dispositivos GC GAA SOI}

\subsubsection{Características dos Dispositivos}

As características físicas dos dispositivos são: espessura da camada de silício $\left(\mathrm{t}_{\mathrm{si}}\right)$ de $80 \mathrm{~nm}$, espessura do óxido de porta $\left(\mathrm{t}_{\mathrm{oxf}}\right)$ de $30 \mathrm{~nm}$, concentração de dopantes na região de canal de $6,8 \times 10^{16} \mathrm{~cm}^{-3}$ (região de ajuste da tensão de limiar), no caso dos dispositivos GC a região menos dopada tem concentração de $1,0 \times 10^{15} \mathrm{~cm}^{-3}$. Esses dispositivos são de três dedos paralelos, cada um com comprimento de $3 \mu \mathrm{m}$ e largura de $3 \mu \mathrm{m}$. 


\subsubsection{Resultados e Discussão}

\subsection{Região Linear de Operação}

A verificação inicial foi realizada através das curvas $I_{D S} \times V_{G F}$ obtidas através de medidas experimentais com transistores GC-GAA SOI nMOSFETs, em diferentes temperaturas e operando na região linear de polarização. É possível notar a ocorrência do ponto ZTC para os diferentes valores da relação de comprimento de canal (LLD/L), Figuras 60 a 76.

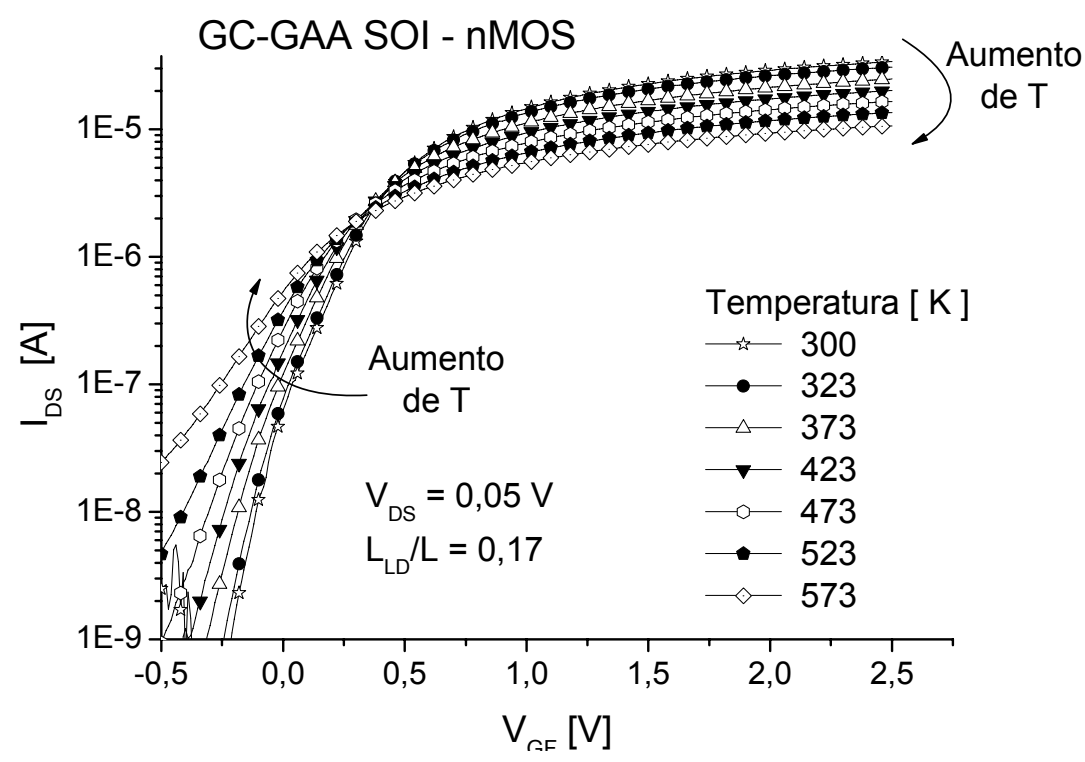

Figura 60: Curvas $I_{D S} \times V_{G F}$ de medidas experimentais do transistor GC-GAA SOI nMOS $\left(L_{L D} / L=0,17\right)$ em diferentes temperaturas para $V_{D S}=0,05 \mathrm{~V}$. 


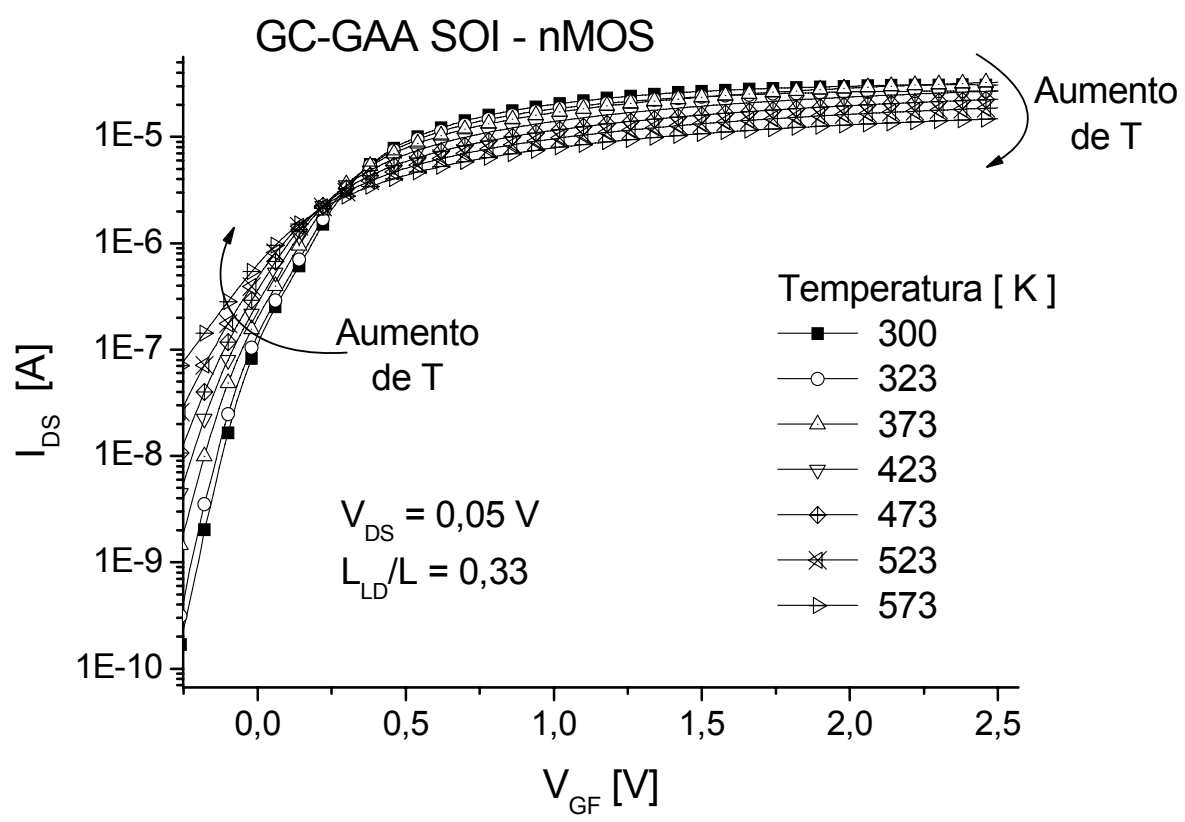

Figura 61: Curvas $I_{D S} \times V_{G F}$ de medidas experimentais do transistor GC-GAA SOI nMOS $\left(L_{L D} / L=0,33\right)$ em diferentes temperaturas para $V_{D S}=0,05 \mathrm{~V}$.

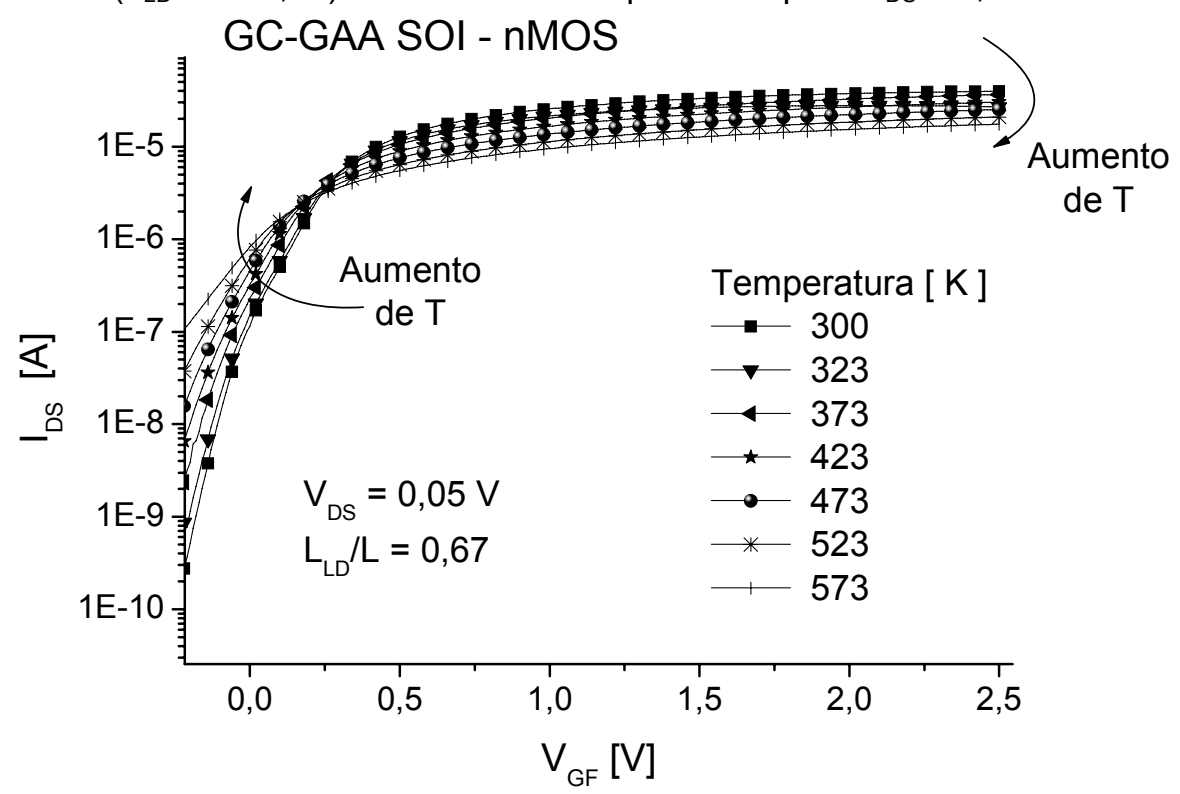

Figura 62: Curvas $I_{D S} \times V_{G F}$ de medidas experimentais do transistor GC-GAA SOI nMOS $\left(L_{L D} / L=0,67\right)$ em diferentes temperaturas para $V_{D S}=0,05 \mathrm{~V}$.

$\mathrm{Na}$ Figura 63 é apresentado o valor de $\mathrm{V}_{\text {ZTC }}$ em função da temperatura, para os dispositivos GC GAA SOI nMOSFETs, que foi obtido experimentalmente e pelo modelo simples proposto operando na região linear através da equação (47), para as diferentes relações de LLD/L. 
Em uma primeira avaliação, os valores do fator $c$, que representa a degradação da mobilidade com a temperatura, que foram utilizados para a obtenção do valor de $\mathrm{V}_{\text {ZTC }}$ para o modelo proposto, foram obtidos do mesmo modo que foi realizado para os dispositivos convencionais planares, ou seja, através do valor da média aritmética do fator $c$ para a faixa de temperatura considerada.

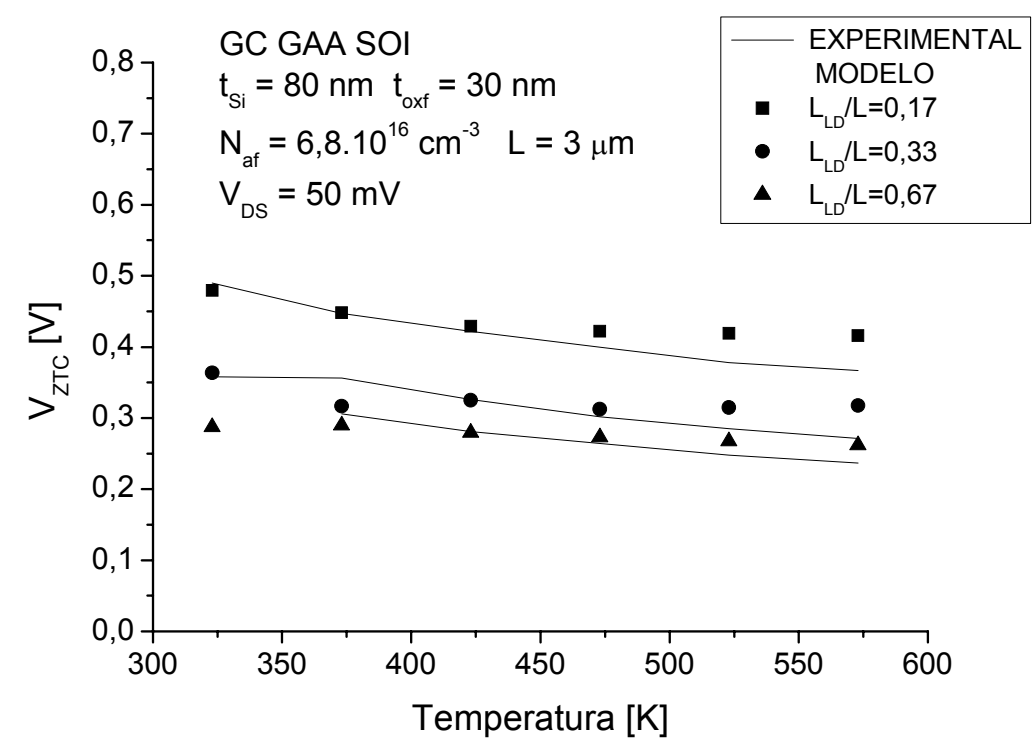

Figura 63: Tensão $V_{Z T C}$ obtido experimentalmente e pelo modelo proposto em função da temperatura para dispositivos GC GAA SOI nMOS operando na região linear $\left(V_{D S}=50 \mathrm{mV}\right)$, para valores de relação de comprimento de canal $\left(L_{L D} / L\right)$ de $0,17,0,33$ e 0,67.

Através da Figura 63 é possível verificar que, esse método não apresentou o mesmo ajuste dos resultados do modelo com os dados experimentais dos dispositivos GC-GAA do ajuste apresentado para os dispositivos convencionais planares. Essa diferença apresentada indica que a degradação da mobilidade com a temperatura, verificada pelo fator $c$, sofre uma variação diferente. Para a verificação dessa diferença, avaliou-se a variação de $V_{\text {ZTC }}$ com a temperatura para uma das relações $L_{L D} / L$ utilizadas, e com os valores do fator $c$ de 1, 1,5, 2, 2,5 e 3 . 
A Figura 64 ilustra a influência do fator $c$, inserido no modelo $\mathrm{CM}$ proposto através da equação (47), na variação de $\mathrm{V}_{\mathrm{ZTC}}$ com a temperatura, e também pode-se verificar a diferente tendência de variação da curva de $\mathrm{V}_{\mathrm{ZTC}}$ com a temperatura dos dados experimentais.

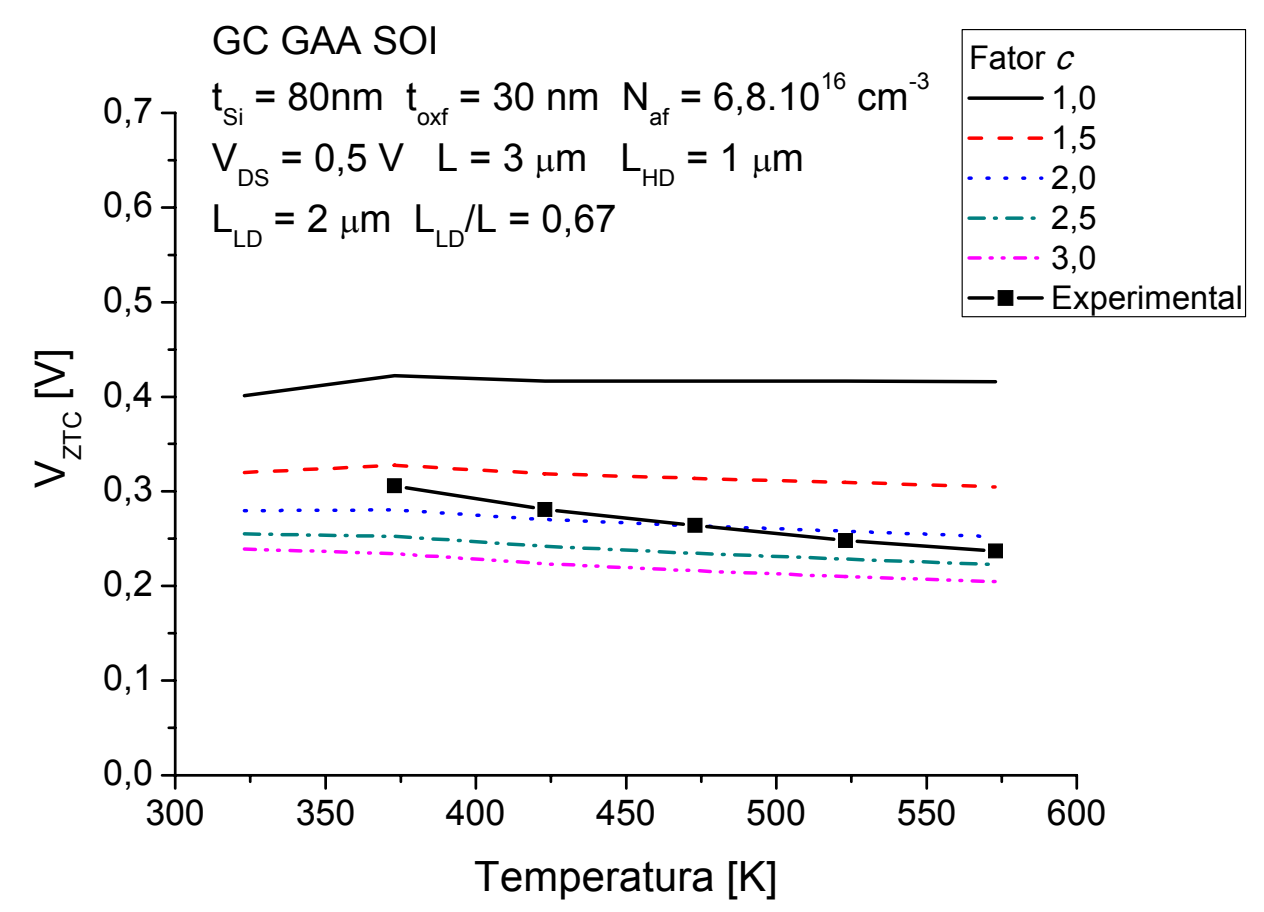

Figura 64: Tensão $V_{\text {ZTC }}$ obtido pelo modelo proposto para dispositivos GC GAA SOI nMOS para uma relação de comprimento de canal $\left(\mathrm{L}_{\mathrm{LD}} / \mathrm{L}\right)$ de 0,67 , operando na região linear $\left(\mathrm{V}_{\mathrm{DS}}=\right.$ $50 \mathrm{mV}$ ), com valores do fator $c$ de $1,1,5,2,2,5$ e 3.

Desta forma, foi verificada a variação do fator c com a temperatura para os dispositivos GC-GAA estudados, Figura 65. Foi adotado a utilização da extrapolação linear das retas obtidas, dispositivos com relações de $L_{L D} / L$ de $0,17,0,33$ e 0,67 , obtendo-se uma equação de reta para a determinação do fator $c$ dependente da temperatura, ou seja, na forma $c=a+b$.(T2), em que a e b são os coeficientes da reta e T2 é a temperatura de medida. 


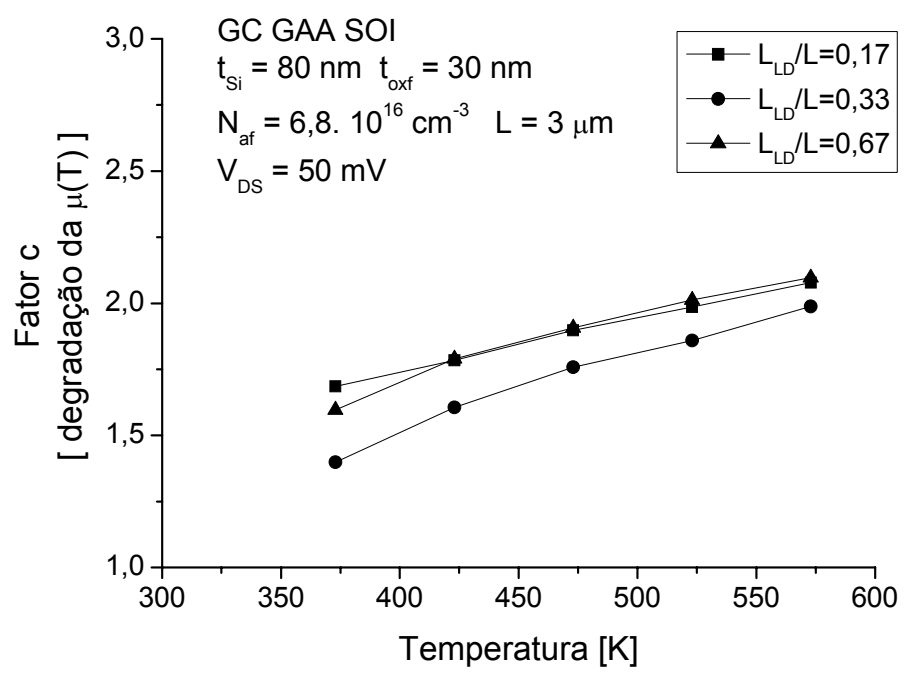

Figura 65: Variação do fator de degradação da mobilidade com a temperatura (Fator $C$ ).

A proposta para o valor do fator c em função da temperatura, como descrito anteriormente, foi utilizada na equação (47) e obtido o valor de $V_{\text {ZTC }}$ para as relações de $\mathrm{L}_{\mathrm{LD}} / \mathrm{L}$ analisadas, Figura 66. Através da Figura 66, é possível verificar um melhor ajuste dos resultados do modelo proposto com os dados experimentais para as relações de $\mathrm{L}_{\mathrm{LD}} / \mathrm{L}$ analisadas.

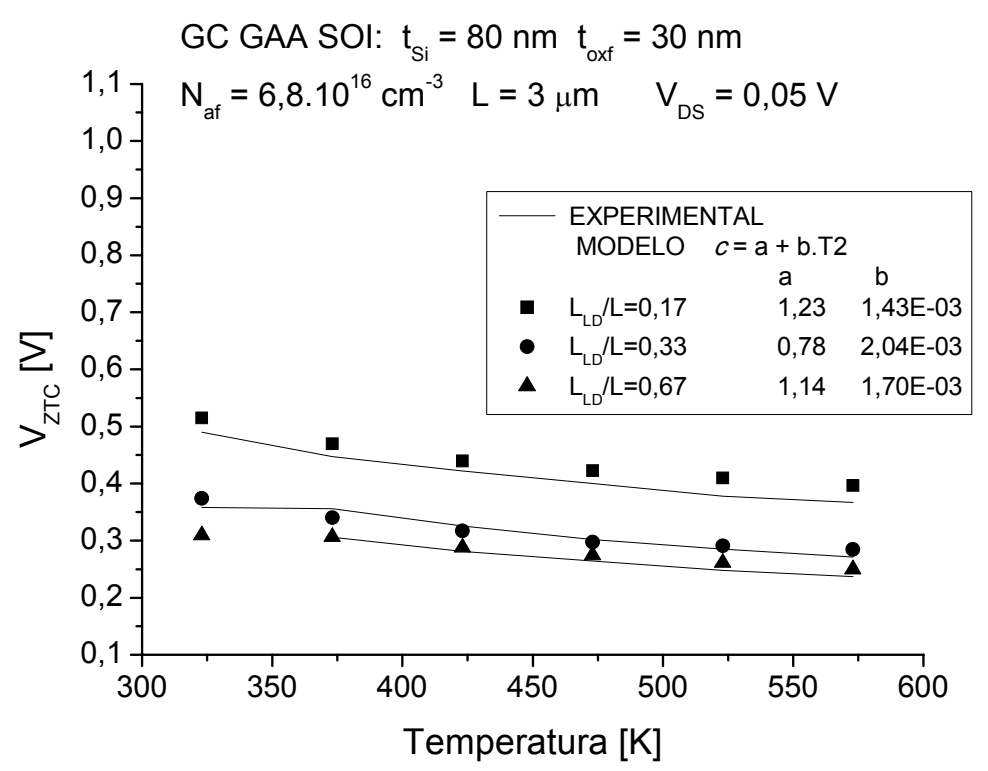

Figura 66: Tensão de polarização no ponto ZTC $\left(\mathrm{V}_{\mathrm{ZTC}}\right)$ obtido experimentalmente em função da temperatura para dispositivos GC GAA SOI nMOS operando na região linear ( $V_{D S}$ $=50 \mathrm{mV})$, para valores de relação de comprimento de canal $\left(\mathrm{L}_{\mathrm{LD}} / \mathrm{L}\right)$ de $0,17,0,33$ e 0,67 . 
A Figura 67 apresenta 0 valor de $\mathrm{V}_{\text {ZTC }}$, de dados obtidos experimentalmente para os dispositivos GC-GAA SOI nMOSFETs, em função do comprimento de canal $L_{H D}$ de 1,2 e 2,5 $\mu \mathrm{m}$, ou seja, para as relações de comprimento de canal ( $\left.L_{L D} / L\right)$ de $0,67,0,33$ e 0,17 . Pode-se observar a influência no valor do ponto invariante com a temperatura ( $\left.V_{\text {ZTC }}\right)$ com a variação do comprimento de canal do dispositivo. Através dos dados analisados verifica-se a diminuição no valor de $\mathrm{V}_{\text {ZTC }}$ com a redução do comprimento de canal, indicando a ocorrência do efeito de canal curto (SCE - short-channel effect), uma vez que este efeito é observado no comportamento da tensão de limiar, Figura 68. Como conseqüência, o mesmo efeito ocorre na tensão de polarização no ponto ZTC $\left(\mathrm{V}_{\mathrm{ZTC}}\right)$, devido a sua dependência direta com a tensão de limiar, como mostra o modelo $\mathrm{CM}$ proposto na equação (47).

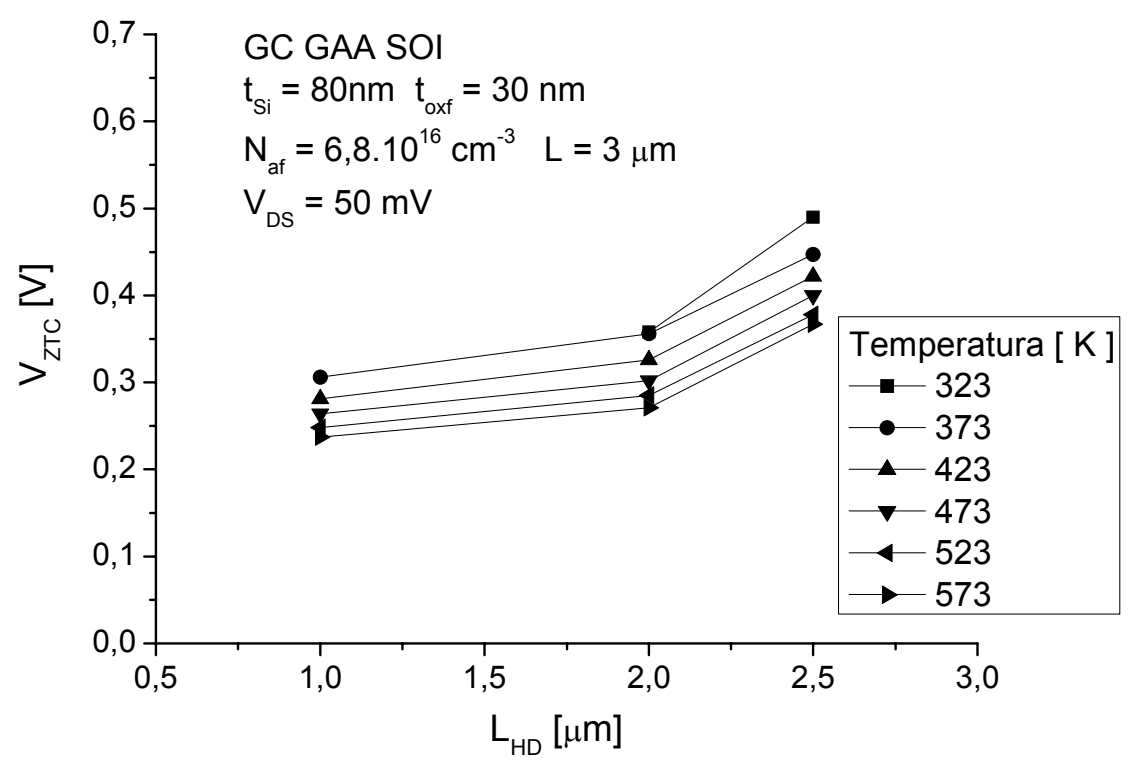

Figura 67: Tensão de polarização no ponto $Z T C\left(V_{Z T C}\right)$ em função do comprimento de canal da região altamente dopada ( $\mathrm{L}_{\mathrm{HD}}$ ) obtido experimentalmente, para dispositivos GC GAA SOI $\mathrm{nMOS}$ operando na região linear $\left(\mathrm{V}_{\mathrm{DS}}=50 \mathrm{mV}\right)$. 


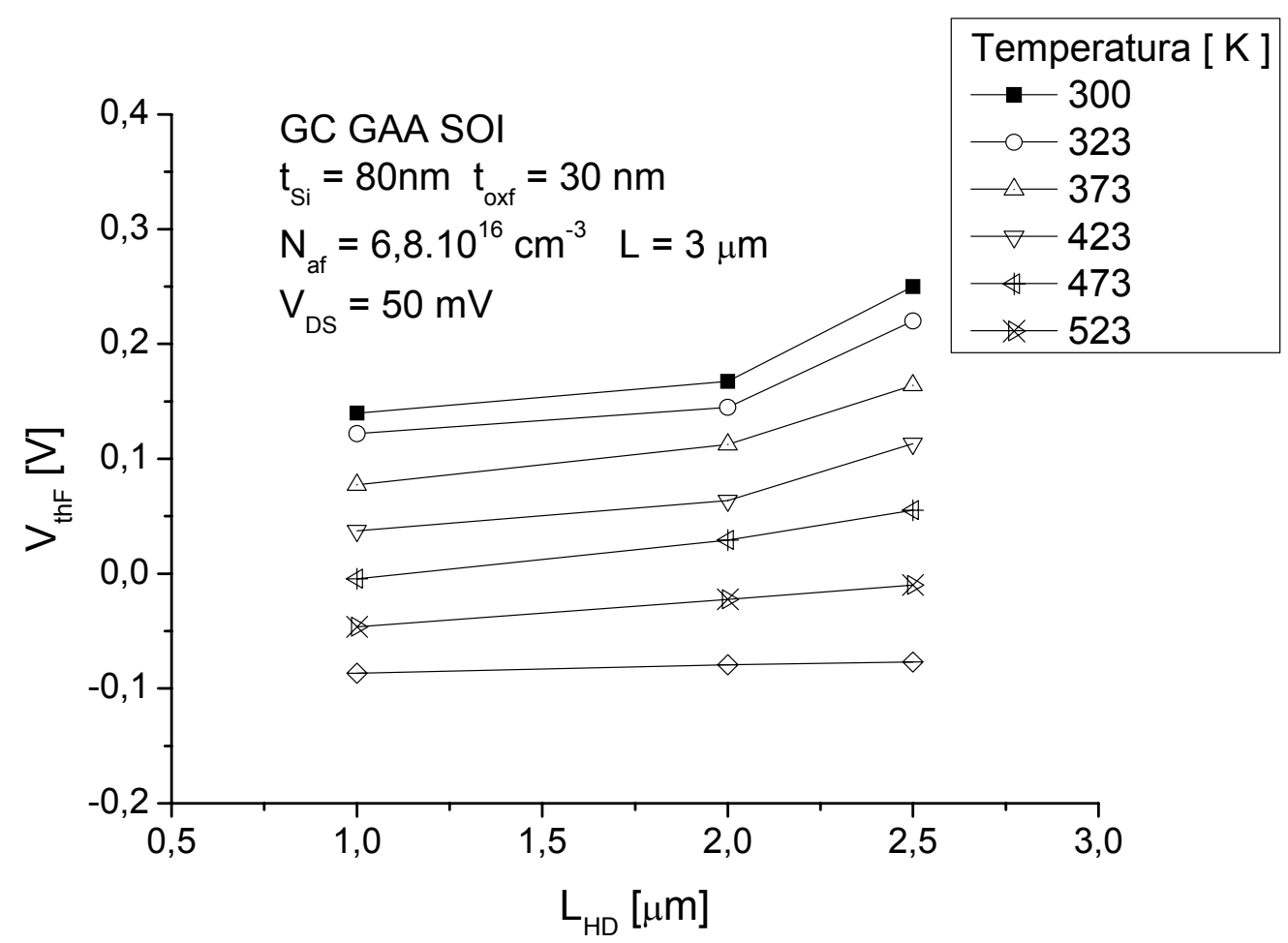

Figura 68: Dados experimentais: Variação da Tensão de limiar $\left(V_{\text {thF }}\right)$ em função do comprimento de canal da região altamente dopada $\left(L_{H D}\right)$ para dispositivos GC GAA SOI nMOS, para temperaturas de 300 a $573 \mathrm{~K}$.

\subsection{Região de Saturação}

Operando na região de saturação de polarização, as curvas $I_{D S} \times V_{G F}$ foram obtidas da mesma forma que os dados extraídos com o transistor polarizado em regime linear, ou seja, através de medidas experimentais com os transistores GC GAA nMOSFETs sob diferentes temperaturas, da Figura 69 a Figura 71. Nas figuras é possível observar a ocorrência do ponto ZTC, para as relações de $L_{L D} / L$ de $0,17,0,33$ e 0,67. 


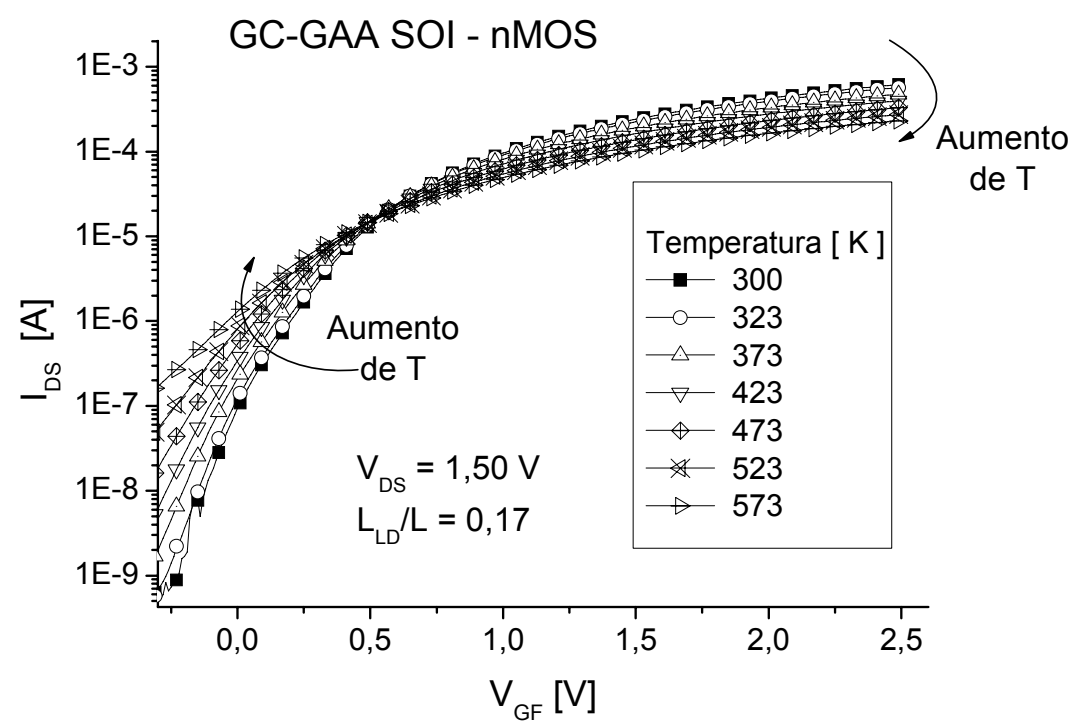

Figura 69: Curvas $I_{D S} \times V_{G F}$ de medidas experimentais do transistor GC-GAA SOI nMOS $\left(L_{L D} / L=0,17\right)$ em diferentes temperaturas para $V_{D S}=1,5 \mathrm{~V}$.

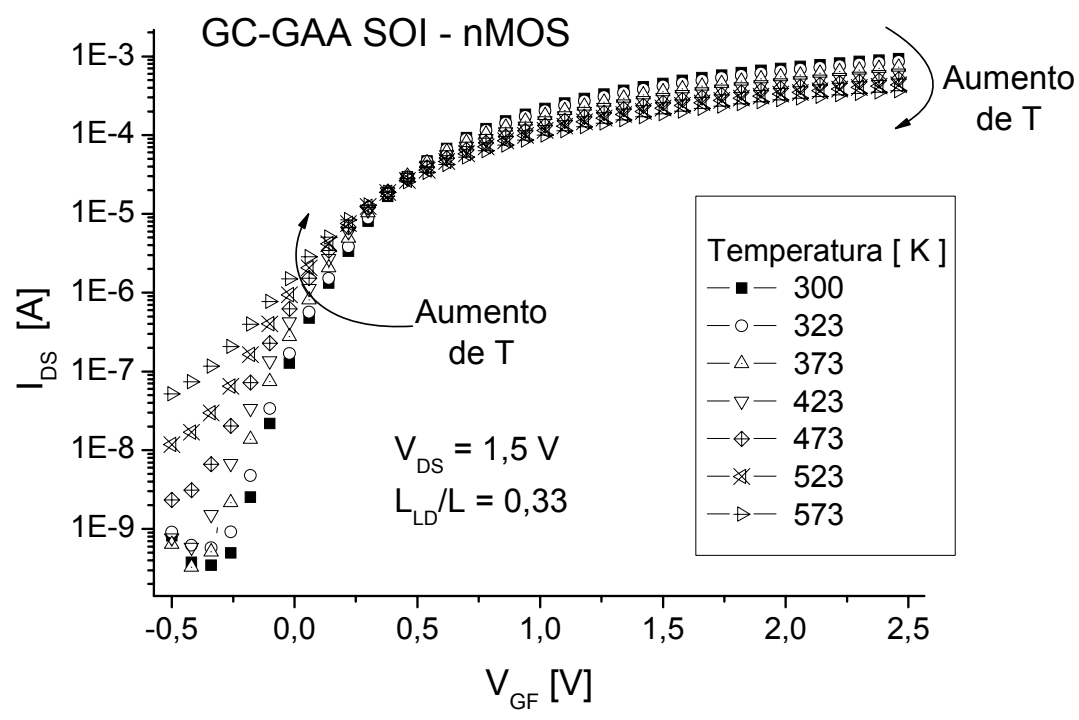

Figura 70: Curvas $I_{D S} \times V_{G F}$ de medidas experimentais do transistor GC-GAA SOI nMOS $\left(L_{\mathrm{LD}} / \mathrm{L}=0,33\right)$ em diferentes temperaturas para $V_{\mathrm{DS}}=1,5 \mathrm{~V}$. 


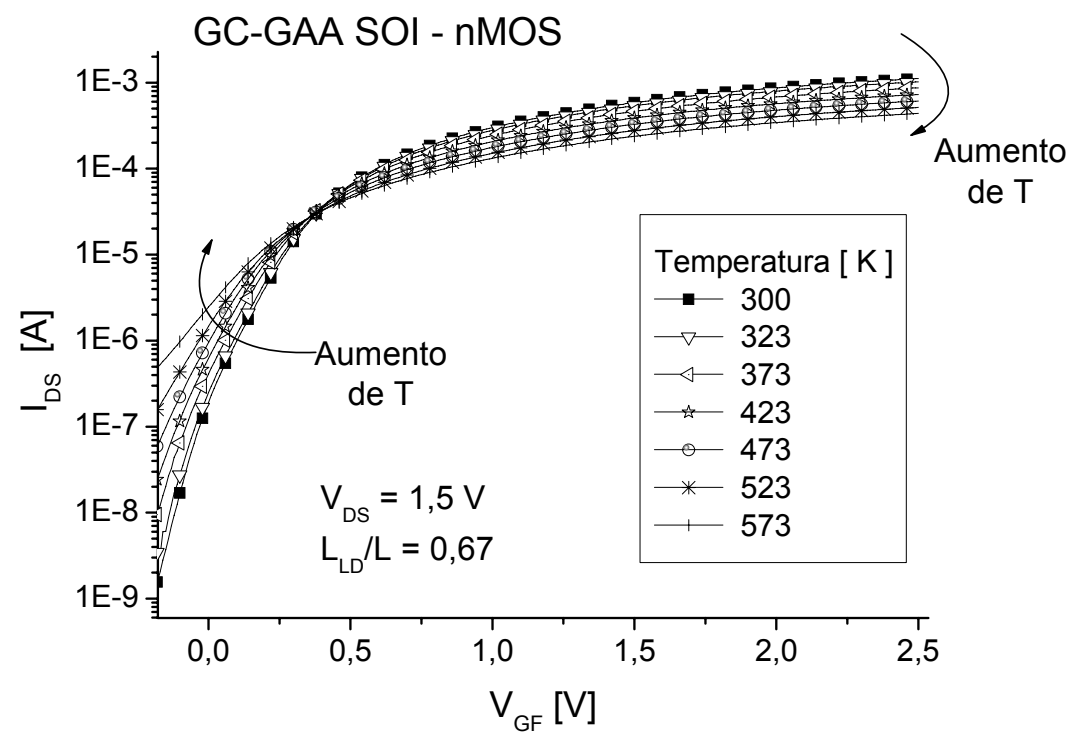

Figura 71: Curvas $I_{D S} \times V_{G F}$ de medidas experimentais do transistor GC-GAA SOI nMOS $\left(L_{L D} / L=0,67\right)$ em diferentes temperaturas para $V_{D S}=1,5 \mathrm{~V}$.

$\mathrm{Na}$ Figura 72 é apresentado o valor de $V_{\text {ZTC }}$ em função da temperatura, para os dispositivos GC GAA SOI nMOSFETs, que foi obtido experimentalmente e pelo modelo simples proposto operando na região de saturação através da equação (51), para as diferentes relações de $L_{L D} / L$

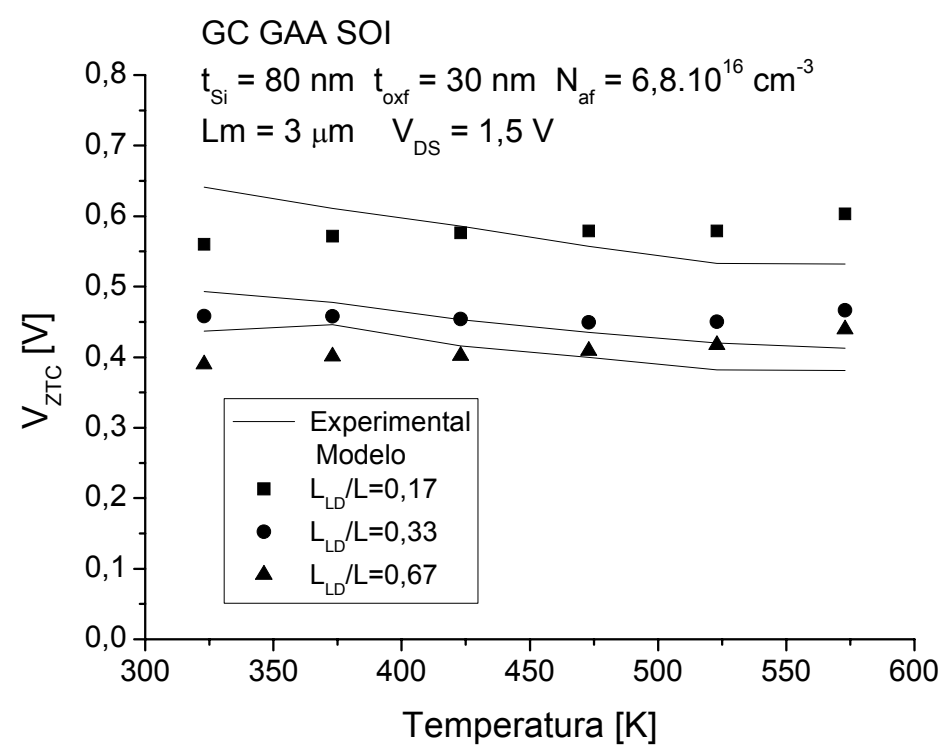

Figura 72: Tensão $V_{Z T C}$ obtido experimentalmente e pelo modelo proposto em função da temperatura para dispositivos GC GAA SOI nMOS operando na região de saturação $\left(V_{D S}=\right.$ $1,5 \mathrm{~V})$, para valores de relação de comprimento de canal $\left(L_{L D} / L\right)$ de $0,17,0,33$ e 0,67. 
Os resultados apresentados na Figura 72 , do valor de $\mathrm{V}_{\text {ZTC }}$ obtido pelo modelo proposto, foram obtidos do mesmo modo como realizado para dispositivos convencionais planares, ou seja, através do valor da média aritmética do fator $c$ para a faixa de temperatura analisada. Entretanto, os resultados do modelo proposto, com o transistor operando em regime de saturação, não apresentaram um bom ajuste com dados experimentais para os dispositivos GC-GAA.

Foi realizado também a avaliação do fator $c$, inserido no modelo proposto através da equação (51), para os dispositivos operando em regime de saturação. A Figura 73 mostra apresenta a variação de $V_{\text {ZTC }}$ com a temperatura para uma das relações LDD $_{L} / \mathrm{L}$ utilizadas, comparando os resultados com valores do fator $c$ de 1, 1,5,2, 2,5 e 3. Na Figura 73 também nota-se a curva da variação de $\mathrm{V}_{\text {ZTC }}$ com a temperatura dos dados experimentais. Da mesma forma que observado com os dispositivos operando em regime linear, verifica-se a tendência diferente de sua variação.

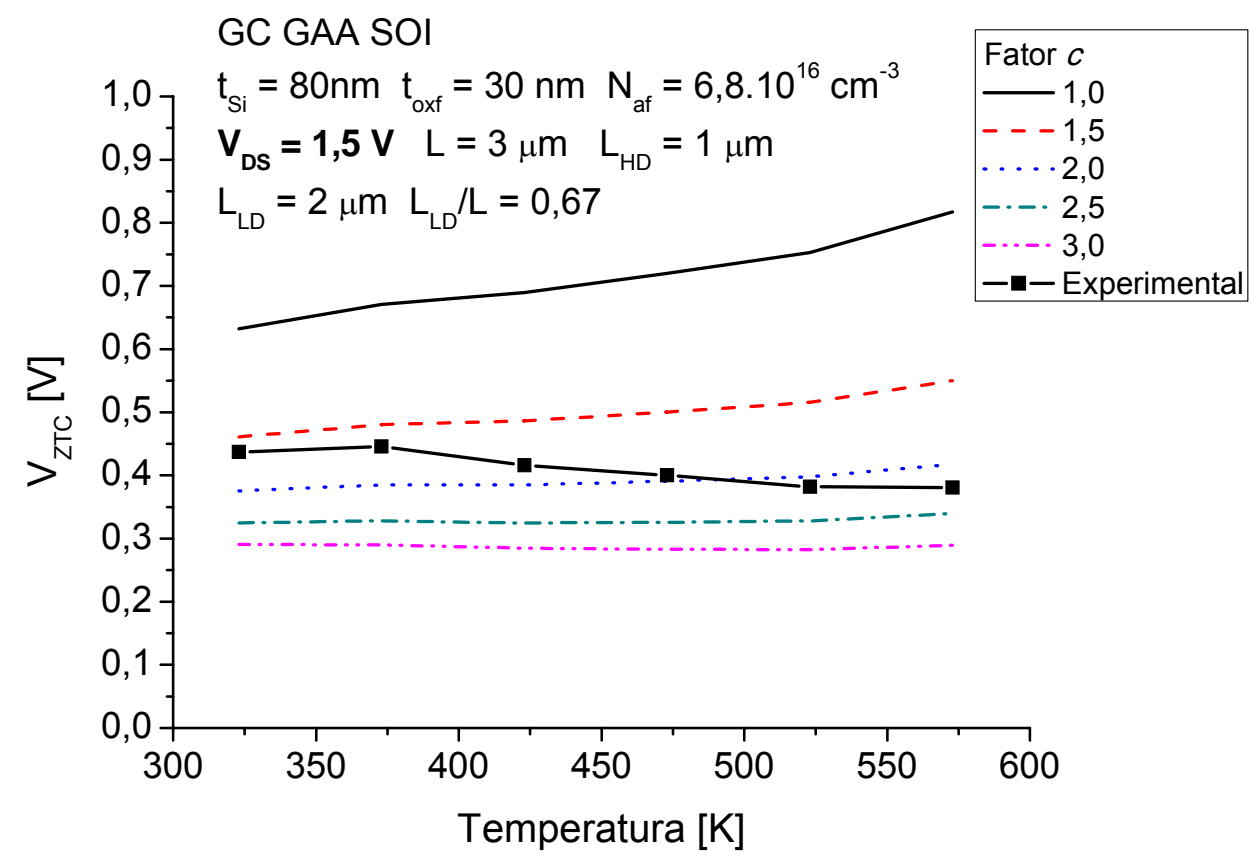

Figura 73: Tensão $V_{\text {ZTC }}$ obtido pelo modelo proposto para dispositivos GC GAA SOI nMOS para uma relação de comprimento de canal $\left(L_{L D} / L\right)$ de 0,67 , operando na região de saturação $\left(V_{D S}=1,5 \mathrm{~V}\right)$, com valores do fator $c$ de 1, 1,5, 2, 2,5 e 3 . 
A partir da variação do fator $c$ com a temperatura, Figura 74 , para os dispositivos GC GAA com relações de $\mathrm{L}_{\mathrm{LD}} / \mathrm{L}$ de 0,17 , 0,33 e 0,67, operando em regime de saturação, obteve-se a extrapolação linear das curvas obtidas para os dispositivos citados. Da mesma forma que a análise realizada para a região de linear, obteve-se uma equação de reta para o fator c dependente da temperatura.

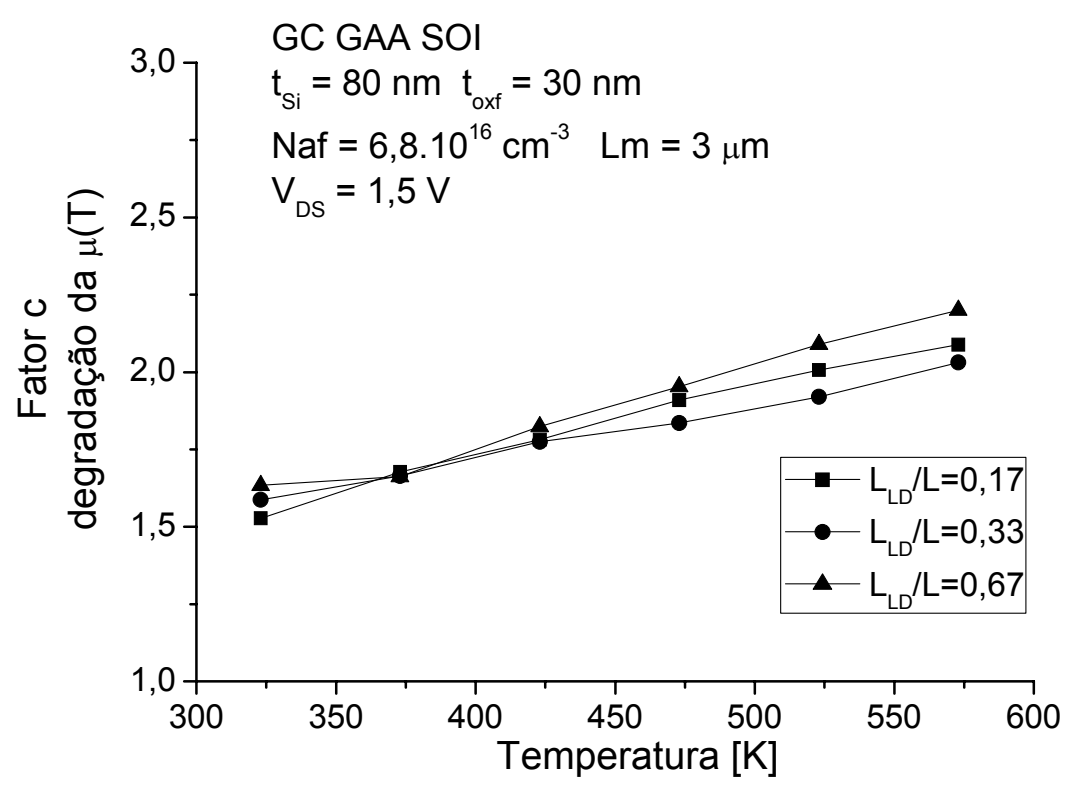

Figura 74: Variação do fator de degradação da mobilidade com a temperatura (Fator $C$ ) para GC GAA SOI em regime de saturação.

Utilizando a proposta para determinação do valor do fator c em função da temperatura, inserindo na equação (51), obteve-se o valor de $\mathrm{V}_{\mathrm{ZTC}}$ para as relações de $L_{L D} / L$ analisadas, Figura 75 . Na mesma figura, é possível observar um melhor ajuste dos resultados do modelo proposto com os dados experimentais para as relações de $L_{L D} / L$ analisadas. 


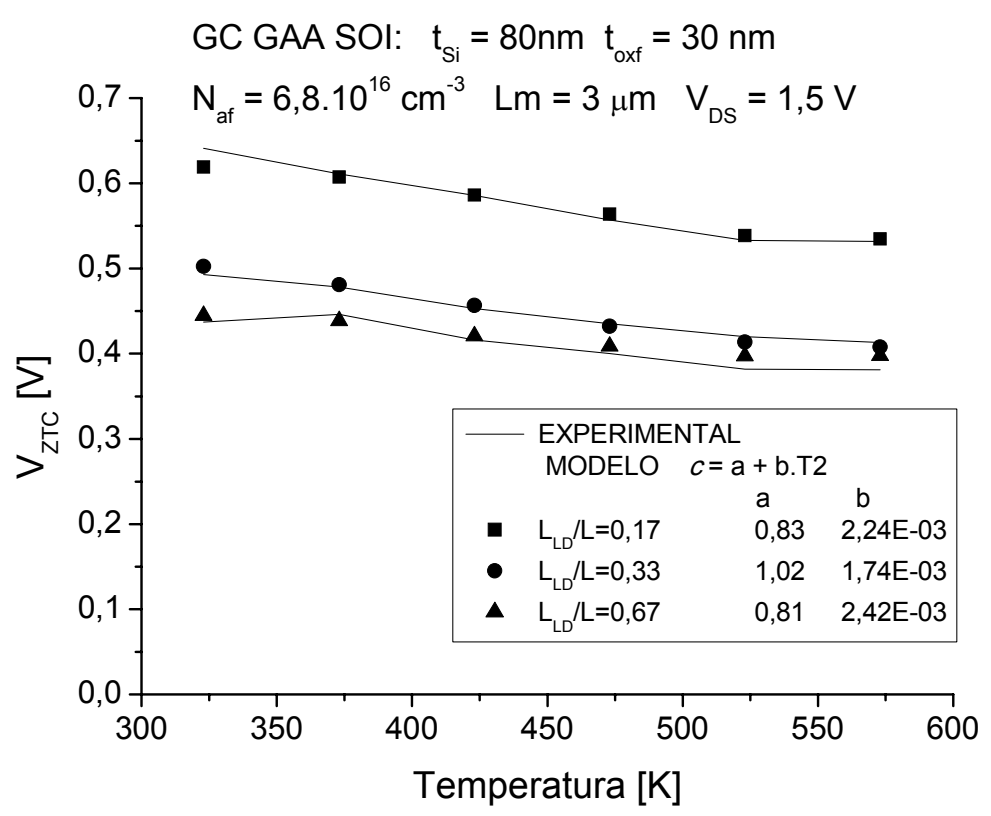

Figura 75: Tensão de polarização no ponto ZTC $\left(\mathrm{V}_{\mathrm{ZTC}}\right)$ obtido experimentalmente em função da temperatura para dispositivos GC GAA SOI nMOS operando na região de saturação $\left(V_{D S}=1,5 \mathrm{~V}\right)$, para valores de relação de comprimento de canal $\left(L_{L D} / L\right)$ de 0,17 , 0,33 e 0,67 .

Realizou-se também a verificação da influência no valor do ponto invariante com a temperatura $\left(\mathrm{V}_{\mathrm{ZTC}}\right)$ com a variação do comprimento de canal do dispositivo, operando em regime de saturação.

A Figura 76 apresenta os dados obtidos experimentalmente para os dispositivos GC GAA SOI nMOSFETs com $L_{H D}$ de 1, 2 e 2,5 $\mu \mathrm{m}$, ou seja, para as relações de comprimento de canal $\left(L_{L D} / L\right)$ de 0,67, 0,33 e 0,17. Analisando a variação de $V_{\text {ZTC }}$ em função do comprimento de canal, verificase a diminuição no valor de $\mathrm{V}_{\mathrm{ZTC}}$ com a redução do comprimento de canal, como observado para o regime linear de operação. Portanto, este efeito também indica a ocorrência do efeito de canal curto (SCE - short-channel effect) no comportamento da tensão de limiar, influênciando diretamente na tensão de polarização no ponto $\mathrm{ZTC}\left(\mathrm{V}_{\mathrm{ZTC}}\right)$ no regime de polarização de saturação. 


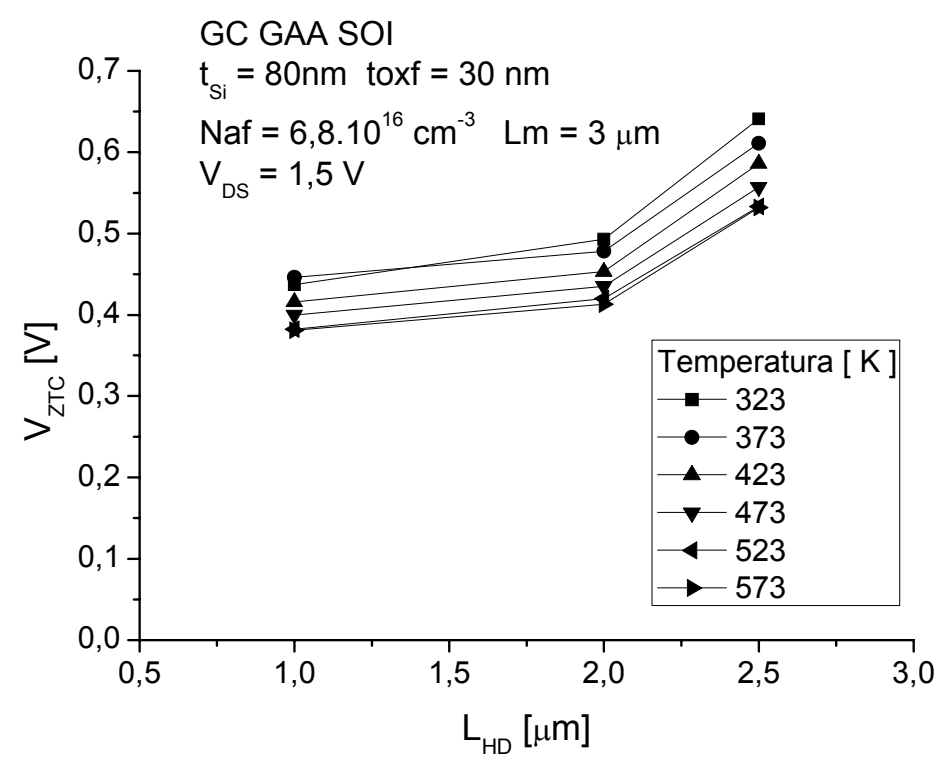

Figura 76: Tensão de polarização no ponto ZTC $\left(\mathrm{V}_{\mathrm{ZTC}}\right)$ em função do comprimento de canal da região altamente dopada ( $\mathrm{L}_{\mathrm{HD}}$ ) obtido experimentalmente, para dispositivos GC GAA SOI $n M O S$ operando na região de saturação $\left(V_{D S}=1,5 \mathrm{~V}\right)$. 


\section{Conclusões}

Neste trabalho foi apresentado um modelo analítico simples (modelo CM: Camillo-Martino) para a polarização no Ponto Invariante com a Temperatura (ZTC), para análise dos principais fatores que influênciam na obtenção deste ponto, em dispositivos SOI MOSFETs operando nas regiões linear e de saturação, dentro do intervalo de temperatura de 20 a $300^{\circ} \mathrm{C}$.

$\mathrm{O}$ modelo $\mathrm{CM}$ foi usado para estudar a influência do fator de degradação da mobilidade com a temperatura $(c)$ na tensão de polarização de porta no ponto ZTC $\left(\mathrm{V}_{\mathrm{ZTC}}\right)$ em dispositivos SOI MOSFETs no modo PD (Parcialmente Depletado) e FD (Totalmente Depletado).

As mudanças em $V_{\mathrm{ZTC}}$, no intervalo de temperatura investigado, mostraram a existência de uma dependência da degradação da mobilidade com a temperatura, que apresentou maior influência nos dispositivos parcialmente depletados (PD).

Analisando os dados experimentais com polarização na região linear de operação, observa-se que para os dispositivos PD com o aumento da temperatura, o valor de $V_{\text {ZTC }}$ também aumenta, mas ocorre uma leve diminuição nos dispositivos FD. Esta diferença é devida aos valores obtidos experimentalmente do fator de degradação da mobilidade com a temperatura $(c)$, que é maior nos dispositivos FD $(1,69)$ do que nos dispositivos PD $(1,46)$. Um bom ajuste dos valores foi obtido apesar das simplificações usadas no modelo $\mathrm{CM}$ proposto.

A influência do fator de degradação da mobilidade com a temperatura (c) na tensão de polarização de porta no ponto ZTC $\left(\mathrm{V}_{\mathrm{ZTC}}\right)$, em dispositivos SOI MOSFETs no modo PD e FD foi investigada através do modelo CM proposto operando na região de saturação. Os resultados foram comparados com os dados experimentais, obtendo um bom ajuste para o dispositivo PD com $\mathrm{c}=1,46$. 
O modelo $\mathrm{CM}$ proposto também foi utilizado para o estudo da influência da polarização da tensão de dreno $\left(V_{D S}\right)$ e do comprimento de canal (L) de dispositivos SOI MOSFETs na polarização da tensão de porta no ponto ZTC, nas regiões de operação linear e de saturação. Os resultados obtidos através do modelo $\mathrm{CM}$ proposto mostraram um bom ajuste com os valores extraídos experimentalmente, em ambas as regiões de operação dos dispositivos analisados.

A tensão de polarização no ponto ZTC $\left(\mathrm{V}_{\mathrm{ZTC}}\right)$, na faixa de temperatura estudada, para os dispositivos SOI nMOS apresentou um menor valor operando na região de saturação do que na região linear, que pode ser previsto pelo modelo CM. E essa diferença tornou-se mais pronunciada para dispositivos com menor comprimento de canal para ambos os tipos de dispositivos, SOI $\mathrm{n}$ e pMOS. Foi observado o efeito reverso de canal curto (RSCE) sobre o comportamento da tensão de limiar $\left(\mathrm{V}_{\text {thF }}\right)$ e conseqüentemente foi notado sua influência em $V_{Z T C}$, observado pela variação de $V_{\text {ZTC }}$ em função do comprimento de canal, para os dispositivos nMOS.

Observando a variação de $\mathrm{V}_{\mathrm{ZTC}}$ com a tensão de dreno $\mathrm{V}_{\mathrm{DS}}$, nota-se uma diminuição no valor de $V_{\text {ZTC }}$ para altos valores de $V_{D S}$, sendo possivelmente devido à redução do valor da tensão de limiar pela ocorrência do efeito DIBL e o impacto da alta tensão de dreno na mobilidade dos portadores no canal dos dispositivos.

O modelo CM proposto também foi aplicado para outras tecnologias de fabricação dos dispositivos SOI MOSFETs, além da tecnologia convencional planar, foram estudados para dispositivos fabricados na tecnologia GC SOI e GC GAA SOI.

Para os dispositivos fabricados na tecnologia GC SOI os resultados obtidos através do modelo proposto, apresentaram um bom ajuste com os valores extraídos experimentalmente, em ambas regiões de operação.

Analisando os dados experimentais dos dispositivos GC SOI, também foi verificada a redução no valor de $\mathrm{V}_{\mathrm{ZTC}}$ para menores valores do comprimento de canal $\left(\mathrm{L}_{H \mathrm{D}}\right)$, influência do comportamento da tensão de 
limiar, que sofre com o efeito de canal curto. O modelo proposto mostra que os efeitos sobre a tensão de limiar influênciam diretamente na tensão $V_{\text {ZTC. }}$.

Para os dispositivos fabricados na tecnologia GC-GAA SOI os resultados obtidos através do modelo proposto, aplicado do mesmo modo que foi para os dispositivos convencionais, não apresentaram inicialmente um bom ajuste com os valores extraídos experimentalmente. Entretanto, após análise da variação do fator $\mathrm{c}$, que indica a degradação da mobilidade com a temperatura, foi verificado que o seu valor inserido no modelo, ou seja, o valor da sua média aritmética para a faixa de temperatura analisada, não representava uma boa aproximação.

Com análise da variação do fator $\mathrm{c}$ em função da temperatura, para as diferente relações de $L_{L D} / L$, foi possível aproximar o seu valor inserido no modelo CM proposto, equações (47) e (51), para uma equação de reta obtida do ajuste linear do fator $\mathrm{c}$ em função da temperatura. Desta maneira foi obtido um bom ajuste dos resultados do modelo CM com os dados experimentais para os dispositivos GC-GAA SOI, com relações de $\mathrm{L}_{\mathrm{LD}} / \mathrm{L}$ de $0,17,0,33$ e 0,67 , para ambas as regiões de polarização.

Foram analisados dados experimentais dos dispositivos GC-GAA SOI, e verificou-se a redução no valor de $V_{\text {ZTC }}$ para menores valores do comprimento de canal $\left(\mathrm{L}_{\mathrm{HD}}\right)$, indicando a ocorrência do efeito de canal curto (SCE - short-channel effect), uma vez que este efeito foi observado no comportamento da tensão de limiar, e desta forma influênciando diretamente na tensão $\mathrm{V}_{\mathrm{ZTC}}$.

Como sugestão de seqüência de trabalho recomenda-se estudar a aplicação do modelo $\mathrm{CM}$ para dispositivos SOI de Múltiplas Portas, considerando-se a dificuldade para esses dispositivos em se obter o valor da mobilidade e o fato da degradação da mobilidade com a temperatura (fator c) não apresentar valores próximos a média aritmética, desta forma não permitindo a aproximação de um valor constante, como no caso dos dispositivos SOI planares. 


\section{Referências Bibliográficas}

\footnotetext{
${ }^{1}$ IZUMI, K. Proceeding of the Fourth International Symposium on Silicon On Insulator Technology and Devices. v.90-6, p.3, 1990.

${ }^{2}$ COLINGE, J. P. "Thin-film SOI technology the solution to many submicron CMOS problems”, IEDM Tech. Dig., p.817-820, 1989.

3 HEMMENT, P. L. F. Semiconductor-on-insulator and thin-film transistor technology. MRS Symposium Proceedings, ed. Chiang, Geis e Pfeiffer, v. 53, p. 207, 1986.
}

${ }^{4}$ SKORUPA, W. et al. Properties of buried insulating layers in silicon formed by high dose implantation at $60 \mathrm{keV}$. Nucl. Instr. and Meth. in Physics Research, v.B32, p.440, 1998.

5 BROWN, R. B., TENY, F. L. e WU, K.-C., "High temperature microelectronies-expanding the applications for smart sensors," IEDM Tech. Dig., 1987, pp. 274-277.

6 NIEBERDING, W. C. e POWELL, J. A., "High-temperature electronic requirements in aeropropulsion systems," IEEE Trans. Indus. Elec $\neg$ tron., vol. IE-29, pp. 103-106, 1982.

7 DRAPER, B. L. e PALMER, D. W., "Extension of high-temperature electronics," IEEE Trans. Componen., Hybrids Manufact. Tech $\neg n o l .$, vol. CHMT-2, pp. 399-404,1979.

8 KRULL, W. e LEE, J., "Demonstration of benefits of SOI for high temperature operation," IEEE SOI/SOS Technolᄀogy Workshop, SI. Simons Island, GA. 1988.

${ }^{9}$ MASZARA, W. P. Proceedings of the Fourth International Symposium on Silicon On Insulator Technology and Devices, v.90-6, p.199, 1990.

10 COLINGE, J. P. Thin-film SOI devices: A perspective. Microeletronic Engineering, v.8, p.127, 1988.

${ }^{11}$ YOSHIMI, M. et al. Observation of mobility enhancement in ultrathin SOI MOSFETs. Eletronics Letters, v. 24, p. 1078, 1988.

12 AUBERTON-HERVÉ. A. J. et al. A CMOS-SOI $1.4 \mu \mathrm{m}$ technology on $1300^{\circ} \mathrm{C}$ annealed SIMOX substrates without epitaxy. Digest of Symposium on VLSI Technology, p.65; 1988. 
${ }^{13}$ YOUNG, K. K.. Short channel effect in fully depleted SOI MOSFETs. IEEE Trans. El. Devices, v. ED-36, p. 399, 1989.

${ }^{14}$ TSIVIDIS, Y. P., Operation and modeling of the MOS transistor, McGrawHill, 1987.

15 SZE, S. M., Physics of Semiconductor Devices. Wiley-Interscince Publication, 2a edição, ed. New York: J. Wiley \& Sons, 1981.

${ }^{16}$ F. S., SHOUCAIR, "Analytical and Experimental Methods for ZeroTemperature-Coefficient Biasing of MOS Transistors", Electron Lett., vol. 25, n. 17, pp. 1196-1198, 1989.

17 KLAASSEN, F. M. e HES, W., On the temperature coefficient of the MOSFET threshold voltage. Solid-St Electron. v29, p. 787, 1986.

${ }^{18}$ SHOUCAIR, F. S., HWANG, W. e JAIN, P., Electrical characteristics of large scale integration (LSI) MOSFETs at very high temperatures Part I. Microelectron. Reliab. vol. 24, no. 3, p. 465, 1984.

${ }^{19}$ SHOUCAIR, F. S., HWANG, W. e JAIN, P., Electrical characteristics of large scale integration (LSI) MOSFETs at very high temperatures Part II. Microelectron. Reliab. vol. 24, no. 3, p. 487, 1984.

${ }^{20}$ PRIJIC, Z. D., DIMITRIJEV, S. e STOJADINOVIC, N., , Analysis of temperature dependence of CMOS transistors' threshold voltage. Microelectron. Reliab. vol. 31, p. 33, 1991.

21 OSMAN, A. A. et al., "Zero-Temperature-Coefficient Biasing Point of Partially Depleted SOI MOSFET's", IEEE Trans. Electron Devices, vol. 42, no. 9, pp. 1709-1711, 1995.

${ }^{22}$ PRIJIC, Z. D., et al., "The Determination of Zero Temperature Coefficient Point in CMOS Transistors", Microelectron. Reliab. vol. 32, no. 6, pp. 769$773,1992$.

${ }^{23}$ CRISTOLOVEANU, S. Introduction to Silicon On Insulator Materials and Devices. Microelectronic Engineering, n.39, p. 145, 1997.

${ }^{24}$ PLOBL, A.; KRAUTER, G. Silicon-on-insulator: materials aspects and applications, n. 44, p.775, 2000.

${ }^{25}$ BRUEL, M. Silicon on insulator material technology. Electronics Letters, v. 31, p. 1201, 1995. 
${ }^{26}$ COLINGE, J. P. Silicon-on-Insulator Technology: Materials to VLSI, 3ed. Kluwer Academic Publishers, 2004.

${ }^{27}$ KISTLER, N.; WOO, J. Detailed characterization and analysis of the breakdown voltage in fully depleted SOI n-MOSFET's. IEEE Transactions on Electron Devices, v. 41, p. 1217, 1994.

${ }^{28}$ NAKASHIMA,S.; KODATE, J. Integrity of the gate oxide on the thin top $\mathrm{Si}$ layer in ITOX-SIMOX wafers. Proceedings of the IEEE International SOI Conference, p. 119, 1999.

${ }^{29} \mathrm{CHAU}, \mathrm{R}$. et al. A $50 \mathrm{~nm}$ depleted-substrate CMOS transistor (DST). Technical Digest of IEDM, p. 29.1.1, 2001.

${ }^{30}$ DOYLE, B. et al. Transistor Elements for 30nm Physical Gate Lengths and Beyond. Intel Technology Journal, v. 6, n. 2, p. 42, 2002.

${ }^{31}$ GAUTIER, J.; SUN, J. Y. C. On the transient operation of partially depleted SOI NMOSFET's. IEEE Electron Device Letters, v. 16, p. 497, 1995.

32 LIM, H. K.; FOSSUM, J. G. Threshold voltage of thin-film Silicon-oninsulator (SOI) MOSFET's. IEEE Transactions on Electron Devices, v. 30, p. 1244, 1983.

${ }^{33}$ FLANDRE, D. et al. Comparison of SOl versus bulk performances of CMOS micropower single-stage OTAs. Electronics Letters, v. 30, n. 23, p. 1933, 1994.

${ }^{34}$ COLINGE, J. P. Subthreshold slope of thin-film SOI MOSFET's. IEEE Electron Device Letters, v.7, p.244, 1986.

${ }^{35}$ COLINGE, J. P. Reduction of kink effect in thin-film SOI MOSFETs. IEEE Electron Device Letters, v. 9, p. 97, 1988.

${ }^{36}$ BANQUERI, J. et al. A Procedure for the Determination Effective Mobility in an N-MOSFET in the Moderate Inversion Region. Solid-State Electronics, v. 39, n. 6, p. $875,1996$.

${ }^{37}$ KOLHATKARI, J. S. ; DUTTA, A. K. A new mobility model to explain the transconductance overshoot effect observed in ultra-short-channel length MOSFETs. Solid-State Electronics, v. 44, p. 691, 2000.

38 WONG, H.S, et al. Modeling of transconductance degradation and extraction of threshold voltage in thin oxide MOSFET's. Solid-State Electrons, 1987; 30:953. 
39 SCHRODER, D. Semiconductor Material and Device Characterization. Wiley, New York, 1990.

${ }^{40}$ RUSSELL, R.W. and FREDERIKSEN, T.M., 'Automotive and industrial electronic building blocks", IEEE J. Solid-State Circuits, v. SC-7, n. 6, pp. 446-454, 1972.

${ }^{41}$ DAVIS, W.F. and FREDERIKSEN, T.M.. "'A precision monolithic timedelay generator of use in automotive electronic fuel injection systems", IEEE J. Solid-State Circuits, vol. SC-7, no. 6, pp. 462-469, 1972.

42 DAVIS, W.F., "Bipolar design considerations for the automotive environment", IEEE J. Soild-State Circuits, vol. SC-8, no. 6, pp. 419-427, 1973.

${ }^{43}$ Proc. Int. Congr. on Transportation Electronics, SAE, Warrendale, PA, 1980, 1984, 1986.

${ }^{44}$ SHOUCAIR, F.S., "Potential and problems of high temperature electronics and CMOS integrated circuits $\left(25-250^{\circ} \mathrm{C}\right)$ - an overview", Microelectronics Journal vol. 22 no. 2, 1991.

${ }^{45}$ FLEETWOOD, D. M. et al. High-temperature silion-on-insulator electronics for space nuclear power systems: requirements and feasibility. IEEE Trans Nucl Sci.

${ }^{46}$ FLANDRE D., NAZAROV, A. N., HEMMENT P. L. F., Science and technology of semiconductor-on-insulator structures and devices operating in a harsh environment. Kluwer Academic Publisher; 2005.

47 GROESENEKEN, G., et al., IEEE Trans. Temperature Dependence of Threshold Voltage in Thin-Film SOI MOSFETs, Electron Device Lett., v. 11, n. 8, pp. 329-331, 1990.

48 JEON, D. S. e BURK, D. E., A temperature-dependent SOI MOSFET model for high-temperature application $\left(27^{\circ}-300^{\circ} \mathrm{C}\right)$, IEEE Trans. Electron Devices, v. 38, n. 9, pp. 2101-2110, 1991.

${ }^{49}$ KRULL, W. A. e LEE, J. F., Demostration of benefits of SOI for high temperature operation, Proc. IEEE SOS/SOI Technology Workshop (St. Simons Island, GA), p. 69, 1988.

50 SHOUCAIR, F. S., Design considerations in high temperature analog CMOS integrated circuits, IEEE Trans. CHMT-9, n. 3, pp. 242-251, 1986. 
${ }^{51}$ FILANOVSKY, M. e AHMED ALLAM, Mutual Compensation of Mobility and Threshold Voltage Temperature Effects with Applications in CMOS Circuits, IEEE Transactions on Circuits and Systems - I: Fundamental Theory and Applications, v. 48, n. 7, pp. 876-884, Jul. 2001.

52 FILANOVSKY, I.M. e NAJAFIZADEH, L., Zeroing in On a ZeroTemperature Coefficient Point, IEEE 45th Midwest Symposium on Circuits and Systems, v. 1, pp. 271-274, Aug. 2002.

${ }^{53}$ LIM, H. K. e FOSSUM, J. G., Current-voltage characteristics of thin-film SOI MOSFET's in strong inversion. IEEE Trans. Electron Devices, vol. ED31, no. 4, pp. 401-408, 1984.

${ }^{54}$ POWER, J. A. e LANE, W. A., An Enhanced SPICE MOSFET Model Suitable for Analog Applications, IEEE Transactions On Computer-Aided Design, pp. 1418-1425, 1992, 1992.

${ }^{55}$ COLINGE, J.P. e COLINGE, C. A., Physics of Semiconductor Devices, 1st edition, Kluwer Academic Publishers, Boston, 2002.

${ }^{56} \mathrm{LIOU}, \mathrm{J}$. et al., Analysis and Design of MOSFETs, Kluwer, Dordrecht, MA, 1998

${ }^{57}$ CAMILLO, L. M. et al., "The Temperature Mobility Degradation Influence on the Zero Temperature Coefficient of Partially and Fully Depleted SOI MOSFETs", Micr. Journal, vol. 37, 2006, 952-957.

${ }^{58}$ OKUMURA, Y. et al., "A novel source-to-drain nonuniformly doped channel (NUDC) MOSFET for high current drivability and threshold voltage controllability “, IEDM Tech Dig., 1990, 391-394

${ }^{59}$ HORI, T., "A $0.1 \mu \mathrm{m}$ CMOS technology with tilt-implanted punchthrough stopper (TIPS)", IEDM Tech Dig., 1994, 75-78.

${ }^{60}$ TAUR, Y. et al, "CMOS devices below $0.1 \mu \mathrm{m}$ : how high will performance go?”, IEDM Tech Dig., 1997, 215-218.

${ }^{61} \mathrm{JIN}, \mathrm{W}$. et al., "A physical thermal noise model for SOI MOSFET", IEEE Trans. Electron Devices, vol. 47 (4), 2000, 768-773.

62 LIU, Z. H. et al., "Threshold voltage model for deep-submicrometer MOSFETs", IEEE Trans. Electron. Devices, vol. 40 (1), 1993, 86-95.

${ }^{63}$ G. M. Yeric et al., "A universal MOSFET mobility degradation model for circuit simulation", IEEE Trans. On Computer-Aided Design, vol. 9 (10), 1990, 1123-1126. 
${ }^{64}$ PAVANELLO, M. A.; MARTINO, J.A.; DESSARD, V.; FLANDRE, D.. An Asymmetric Channel SOI nMOSFET for Reducing Parasitic Effects and Improving Output Characteristics. Electrochemical and Solid-State Letters, v.3, n.1, p.50-52, 2000

65 SANTOS, C.D.G dos; Caracterização Elétrica de Transistores SOI de Porta Circundante com Estrutura de Canal Gradual em alta temperatura. São Paulo - Brasil, 2005. /Dissertação de Mestrado - Escola Politécnica da Universidade de São Paulo

${ }^{66}$ COLINGE, J. P.; GAO, M. H.; ROMANO-RODRÍGUEZ, A.; CLAEYS C.. Silicon-on-Insulator "Gate-all-around device". Technical Digest of IEDM, p.595, 1990

67 VANDOOREN, A.; COLINGE, J.P.; FLANDRE, D.. Gate-all-Around OTA's for Rad-Hard and High-Temperature Analog Applications. IEEE Transactions on nuclear science, v. 46, n. 4, p. 1242, 1999.

${ }^{68}$ SUN, S. C. AND PLUMMER, J. D., "Electron Mobility in Inversion and Accumulation Layers Thermally Oxidized Silicon Surfaces", IEEE Trans. Electron Devices, v. 27, p. 1497-1508, 1980. 
APENDICE A - Publicações geradas durante o trabalho

\section{Trabalhos completos publicados em anais de congressos}

MARTINO, J. A. ; CAMILLO, L. M. ; ALMEIDA, L. M. ; SIMOEN, Eddy ; CLAEYS, Cor . Zero-Temperature-Coefficient of Planar and MUGFET SOI Devices. In: 10th IEEE International Conference on Solid-State and Integrated Circuit Technology, 2010, Shanghai, China. 10th IEEE International Conference on Solid-State and Integrated Circuit Technology Proceedings, 2010.

CAMILLO, Luciano Mendes ; MARTINO, J. A. ; SIMOEN, Eddy ; CLAEYS, Cor . Influence of the Drain Bias and Gate Length of Partially Depleted SOI MOSFETs on the ZTC Biasing Point. In: 23rd Symposium on Microelectronics Technology and Devices - SBMicro2008, 2008, Gramado, RS. Microelectronics Technology and Devices SBMicro2008. Pennington, NJ, EUA : The Electrochemical Society, 2008. v. 14. p. 243-252.

BELLODI, M. ; CAMILLO, L. M. ; MARTINO, J. A. ; SIMOEN, Eddy ; CLAEYS, C. . Simple Analytical Model to Study the ZTC Bias Point in FinFETs. In: 211th Electrochemical Society Meeting - Silicon-OnInsulator Technology and Devices 13, 2007, Chicago. Silicon-On-Insulator Technology and Devices 13. Pennington, NJ : The Electrochemical Society, 2007. v. 6. p. 205-209.

BELLODI, Marcello ; MARTINO, J. A. ; CAMILLO, Luciano Mendes ; SIMOEN, Eddy ; CLAEYS, Cor . Influence of the N-Type FinFET Width on the Zero Temperature Coefficient. In: 22st Symposium on Microelectronics Technology and Devices - SBMicro2007, 2007, Rio de Janeiro. Microelectronics Technology and Devices SBMicro2007. Pennington, NJ, EUA : The Electrochemical Society, 2007. v. 9. p. 29-36. 
CAMILLO, Luciano Mendes ; MARTINO, J. A. ; SIMOEN, Eddy ; CLAEYS, Cor . Simple Analytical Model to Study the ZTC Bias Point in PD and FD SOI MOSFETS. In: EUROSOI 2006 - Second Workshop of the Thematic Network on Silicon On Insulator Technology, Devices and Circuits, 2006, Grenoble. EUROSOI 2006 Conference Proceedings, 2006. v. 1. p. 77-78.

CAMILLO, Luciano Mendes ; MARTINO, J. A. ; SIMOEN, Eddy ; CLAEYS, C. . The temperature mobility degradation influence on the ZTC of PD and FD MOSFETS. In: Silicon-on-Insulator Technology and Devices Meeting of the Electrochemical Society, 2005, Quebec. Proceedings of Silicon-onInsulator Technology and Devices Meeting of the Electrochemical Society. Pennington, New Jersey, USA : The Electrochemical Society, Inc, 2005. v. 2005-3. p. 119-124.

Artigos completos publicados em periódicos

CAMILLO, Luciano Mendes ; MARTINO, J. A. ; SIMOEN, Eddy ; CLAEYS, Cor . Investigation of the Gate Length and Drain Bia dependence of the ZTC Biasing Point Instability of N- and P- Channel PD SOI MOSFETs. JICS. Journal of Integrated Circuits and Systems (Ed. Português), v. 4, p. 61-66, 2009.

CAMILLO, Luciano Mendes ; MARTINO, J. A. ; SIMOEN, Eddy ; CLAEYS, C. . The temperature mobility degradation influence on the zero temperature coefficient of partially and fully depleted SOI MOSFETs. Microelectronics Journal, Oxford, v. 37, n. 9, p. 952-957, 2006. 